\title{
Elliptic blowup equations for 6d SCFTs. Part II. Exceptional cases
}

\author{
Jie Gu, ${ }^{a}$ Albrecht Klemm, ${ }^{b, c}$ Kaiwen Sun ${ }^{d}$ and Xin Wang ${ }^{b, e}$ \\ ${ }^{a}$ Département de Physique Théorique et Section de Mathématiques, Université de Genève, \\ Genève, $\mathrm{CH}$-1211 Switzerland \\ ${ }^{b}$ Bethe Center for Theoretical Physics, Universität Bonn, \\ D-53115 Bonn, Germany \\ ${ }^{c}$ Hausdorff Center for Mathematics, Universität Bonn, \\ D-53115 Bonn, Germany \\ ${ }^{d}$ Scuola Internazionale Superiore di Studi Avanzati (SISSA), \\ via Bonomea 265, 34136, Trieste, Italy \\ ${ }^{e}$ Max Planck Institute for Mathematics, \\ Vivatsgasse 7, D-53111 Bonn, Germany \\ E-mail: jie.gu@unige.ch, aklemm@th.physik.uni-bonn.de, ksun@sissa.it, \\ wxin@mpim-bonn.mpg.de
}

ABSTRACT: The building blocks of $6 \mathrm{~d}(1,0)$ SCFTs include certain rank one theories with gauge group $G=\mathrm{SU}(3), \mathrm{SO}(8), F_{4}, E_{6,7,8}$. In this paper, we propose a universal recursion formula for the elliptic genera of all such theories. This formula is solved from the elliptic blowup equations introduced in our previous paper. We explicitly compute the elliptic genera and refined BPS invariants, which recover all previous results from topological string theory, modular bootstrap, Hilbert series, $2 \mathrm{~d}$ quiver gauge theories and $4 \mathrm{~d} \mathcal{N}=2$ superconformal $H_{G}$ theories. We also observe an intriguing relation between the $k$-string elliptic genus and the Schur indices of rank $k H_{G}$ SCFTs, as a generalization of Del ZottoLockhart's conjecture at the rank one cases. In a subsequent paper, we deal with all other non-Higgsable clusters with matters.

Keywords: Conformal Field Models in String Theory, Field Theories in Higher Dimensions, Solitons Monopoles and Instantons, Topological Strings

ARXIV EPRINT: 1905.00864 


\section{Contents}

1 Introduction 1

2 Elliptic non-compact CY 3-folds and generalised blowup equations $\quad 7$

$\begin{array}{lll}2.1 & \text { Geometry of elliptic fibrations } & 7\end{array}$

$\begin{array}{ll}2.2 & \text { Semiclassical partition functions } \\ & 10\end{array}$

$\begin{array}{ll}2.3 & \text { Determination of } r \text { fields } \\ & 13\end{array}$

$\begin{array}{lll}2.4 & \text { De-affinisation } & 16\end{array}$

3 Elliptic blowup equations $\quad 18$

$\begin{array}{ll}3.1 \text { Modularity of elliptic blowup equations } & 19\end{array}$

$\begin{array}{ll}3.2 & \text { Universality of elliptic blowup equations } \\ 3.3 & \text { Unity }\end{array}$

$\begin{array}{lll}3.3 & \text { Unity blowup equations } & 22\end{array}$

$\begin{array}{lll}3.3 .1 & \text { Recursion formulas for elliptic genera } & 23\end{array}$

3.3.2 Uniqueness of recursion formulas 26

$\begin{array}{lll}3.4 & \text { Vanishing blowup equations } & 27\end{array}$

4 Elliptic genera for 6d $(1,0)$ minimal SCFTs $\quad 29$

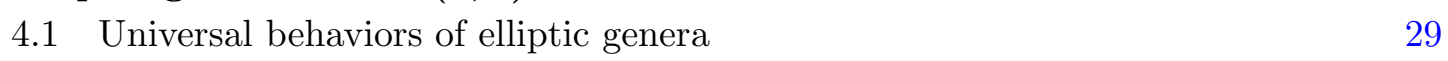

$\begin{array}{lll}4.1 .1 & \text { Universal expansion } & 29\end{array}$

$\begin{array}{lll}\text { 4.1.2 Symmetric product approximation } & 32\end{array}$

4.1.3 Symmetries 34

4.2 Revisiting $G=\mathrm{SU}(3)$ and $\mathrm{SO}(8) \quad 35$

$\begin{array}{ll}4.3 \quad G=F_{4} & 38\end{array}$

$4.4 G=E_{6} \quad 41$

$4.5 \quad G=E_{7} \quad 45$

$4.6 \quad G=E_{8} \quad 48$

5 On the relation with 4d SCFTs of type $H_{G}^{(k)} \quad \mathbf{5 1}$

5.1 Rank $k H_{G}$ theories $\quad 52$

5.2 Hall-Littlewood and Schur indices $\quad 54$

5.3 Rank one: Del Zotto-Lockhart's conjecture $\quad 57$

$\begin{array}{lll}5.4 \text { Rank two } & 60\end{array}$

$\begin{array}{ll}\text { 5.5 Rank three and higher } & 69\end{array}$

6 Conclusion and outlook $\quad 73$

$\begin{array}{ll}\text { A Lie algebraic convention } & 76\end{array}$

B Mirror symmetry for elliptic non-compact Calabi-Yau three-folds $\quad 77$

$\begin{array}{ll}\text { C Geometric data } & 81\end{array}$ 


\section{Introduction}

Six is the highest dimension in which representation theory allows for interacting superconformal quantum theories [1]. Limits of non-perturbative string theory compactifications [2] and in particular the decoupling of gravity in F-theory compactifications to 6d provided the first examples $[3,4]$ and lead recently to a complete classification of geometrically engineered $6 \mathrm{~d}$ superconformal quantum field theories [5-7]. Such a classification in $6 \mathrm{~d}$ is highly desirable, as it might lead by further compactifications, to an exhaustive classification of superconformal theories.

The $6 \mathrm{~d}$ geometry is the one of an — in general desingularised - elliptic fibration with a contractable configuration of desingularised elliptic surfaces fibred over a configuration of curves in the base. In the decoupling limit the volume outside of the configuration of elliptic surfaces is scaled to infinite size, leaving us with an, in general reducible, configuration of complex desingularised elliptic surfaces that can be contracted within a non-compact Calabi-Yau threefold. Because compact components can be contracted such geometries are sometimes called local Calabi-Yau spaces. We will call the above specific ones for short elliptic non-compact Calabi-Yau geometries $X$ and describe them in more detail in section 2.1.

The full topological string partition function on these elliptic non-compact CY geometries has received much attention as it contains important information about protected states of the $6 \mathrm{~d}$ superconformal theories $[3,8]$. Solving the topological string partition function on compact Calabi-Yau manifolds is currently an open problem. On non-compact Calabi-Yau spaces with an $\mathrm{U}(1)_{R}$ isometry a refined topological string partition function $Z\left(\underline{t}, \epsilon_{1}, \epsilon_{2}\right)$, which depends on the Kähler parameters $\underline{t}$ and two $\Omega$ background parameters $\epsilon_{1}, \epsilon_{2}$ is defined as generating function of refined stable pair invariants. ${ }^{1}$ The refinement of the stable pair invariants $[9,10]$ and the relation to the refined BPS invariants $N_{j_{l}, j_{r}}^{\beta} \in \mathbb{N}$ was given in $[11,12]$. Here $\beta \in H_{2}(X, \mathbb{Z})$ is the degree and the half integers $\left(j_{l}, j_{r}\right)$ label a spin representation in the $\mathrm{SU}(2)_{l} \times \mathrm{SU}(2)_{r}$ little group of the 5 d Poincaré group, which can be identified with Lefschetz actions on the moduli space of D2 and D0-branes. On

\footnotetext{
${ }^{1}$ In this section we underline a symbol, if it is a vector. After the introduction section, we drop the underline when there is no risk of confusion.
} 
toric non-compact Calabi-Yau spaces the refined partition function ${ }^{2} Z\left(\underline{t}, \epsilon_{1}, \epsilon_{2}\right)$ can be efficiently calculated by large $N$ techniques $[13],{ }^{3}$ torus localisation [11], the integration of the refined holomorphic anomaly equations [14] and a recursive solution of blowup equations [15] generalized from the Göttsche-Nakajima-Yoshioka K-theoretic blowup equations in the context of $5 \mathrm{~d} \mathcal{N}=1$ supersymmetric gauge theories [16-18].

The class of elliptic non-compact Calabi-Yau relevant for the $(1,0) 6 \mathrm{~d}$ SCFT is nontoric, but has a $\mathrm{U}(1)_{R}$ isometry, and can be viewed as the borderline case for calculating $Z\left(\underline{t}, \epsilon_{1}, \epsilon_{2}\right)$. Since the techniques based on toric localisation and large $N$ expansions fail, two related new methods have been developed. Similar as in heterotic/Type II duality one can calculate ${ }^{4}[8]$ the world-sheet elliptic genus of dual 2 d quiver $(0,4)$ gauge theories with supersymmetric localisation techniques [20,21] leading to Jeffrey-Kirwan integrals. These elliptic genera $\mathbb{E}_{d}\left(\tau, \underline{a}, \underline{m}, \epsilon_{1}, \epsilon_{2}\right)$ transform as a Jacobi form and are identified with the topological string partition function $Z_{d}$ at different winding $d$ of the base [8] up to certain prefactor. The Kähler parameter $\tau$ of the elliptic fibre class becomes the modular parameter while $\left(\epsilon_{1}, \epsilon_{2}\right)$ as well as Kähler parameters $\underline{a}$ of the desingularisations and eventual further sections $\underline{m}$ in the elliptic fibration become elliptic parameters. The refined holomorphic anomaly equations and other B-model techniques also apply and lead to a modular bootstrap approach where different winding contributions $Z_{d}$ are identified with meromorphic Jacobi forms with weight zero and an index, which depends quadratically on the base degree $d$. The $Z_{d}$ are so constrained by modularity, the pole - as well as the refined BPS structure of the topological string that they can be completely reconstructed in many examples [22-24].

In the $2 \mathrm{~d}$ approach one needs for higher $d$ to consider ever more complicated quiver gauge theories, while in the modular approach one has to deal with more and more complicated rings of weak Jacobi forms. For this reason we further develop in this paper the recursive approach based on the elliptic blowup equations [25] for the calculation of $Z\left(\underline{t}, \epsilon_{1}, \epsilon_{2}\right)$ that is further based on a specialisation of the generalized blowup equation in [15] to the elliptic non-compact Calabi-Yau geometries. The main advantage of this approach is that it needs as input only ${ }^{5}$ the classical topological data of $X$, i.e. the classical triple intersection numbers as well as the evaluation of the Chern classes on the elements of the Chow group, and yields with a non ambiguous efficient recursive procedure the string partition function iteratively in the base degree $d$ and for each $d$ exact in $\left(\epsilon_{1}, \epsilon_{2}\right)$ and all other Kähler parameters.

\footnotetext{
${ }^{2}$ The holomorphic all genus partition function $Z(\underline{t}, \lambda)=\exp \left(\sum_{g=0}^{\infty} \lambda^{2 g-2} F_{g}(\underline{t})\right)$ containing the information of all genus Gromov-Witten invariants is obtained as specialisation $\epsilon_{1}=-\epsilon_{2}$ and $\lambda^{2}=-\epsilon_{1} \epsilon_{2}$.

${ }^{3}$ Strictly speaking the refined topological vertex applies directly only to geometries which engineer $\mathcal{N}=2$ gauge theories, as these have the required preferred direction in the torus action. In blow downs and transitions of gauge theories geometries with Chern-Simons terms to geometries which have no immediate gauge theory interpretation, $Z\left(\underline{t}, \epsilon_{1}, \epsilon_{2}\right)$ for the latter can often be recovered [13].

${ }^{4}$ The more supersymmetric case of the M-strings has been pioneered in [19].

${ }^{5}$ This holds for all non-compact CY 3-folds studied in [15] and elliptic non-compact CY 3-folds studied in [25]. For the elliptic non-compact CY 3-folds associated to exceptional gauge symmetry studied in this paper, we also input $Z_{0}$ to the blowup equations, which can be easily calculated from the intersection and the multi-covering of isolated rational curves, see (2.3) below.
} 
Let us first give a short summary of the structure behind the blowup equations in four, five and six dimensions. Non-compact Calabi-Yau spaces with $\mathrm{U}(1)_{R}$ isometry and the (refined) topological string partition function feature prominently in the geometric engineering approach [26] to $5 \mathrm{~d}$ and $4 \mathrm{~d}$ supersymmetric gauge theories as $Z\left(\underline{t}, \epsilon_{1}, \epsilon_{2}\right)$ is related with the K-theoretic extension of Nekrasov's $4 \mathrm{~d}$ gauge theory instanton partition [27] on those non-compact Calabi-Yau spaces [16, 17], which do engineer supersymmetric gauge theories. In the geometric engineering approach, given mirror symmetry, it is physically obvious that world-sheet instantons and space time instantons are related. Simply because the former correct the topological string theory or $\mathcal{N}=2$ supergravity prepotential, while the latter correct the rigid $\mathcal{N}=2$ or Seiberg-Witten prepotential, which is related to the former in a well defined limit in the B-model, that decouples gravity as decribed in [26]. If the geometry engineers five dimensional $\mathrm{U}(N)$ gauge theory, the full correspondence states that the K-theoretic partition function of the latter is identified with $Z\left(\underline{t}, \epsilon_{1}, \epsilon_{2}\right)$ and provides an alternative definition $[16,18]$. The K-theoretic blowup equation for $\mathrm{U}(N)$ theories without or with Chern-Simons terms has been rigorously established in [16] and [17, 18] respectively.

Nakajima and Yoshioka derived the original blowup equations [28] in the context of $4 \mathrm{~d} \mathcal{N}=2$ supersymmetric $\mathrm{SU}(N)$ framed gauge instanton calculus, by studying invariants on moduli space $\widehat{M}(N, k, n)$ of framed torsion free sheaves $(E, \Phi)$ on $\widehat{\mathbb{P}}^{2}$ - a $\mathbb{P}^{1}$ blowup of $\mathbb{P}^{2}$ - via the Atiyah-Bott localization formalism w.r.t. an induced toric action $T=\mathbb{C}^{*} \times \mathbb{C}^{*} \times \mathrm{Gl}_{N}$ on $\widehat{M}(N, k, n)$. Here $\Phi$ is the framing automorphism, $N$ is the rank of $E, n=\left\langle c_{2}(E)-\frac{N-1}{2 N} c_{1}^{2}(E),\left[\widehat{\mathbb{P}}^{2}\right]\right\rangle$ and $k=-\left\langle c_{1}(E),\left[\mathbb{P}^{1}\right]\right\rangle$. A general feature of this calculation is that the Euler class of the tangent space of $\widehat{M}(N, k, n)$ at all relevant fix loci is always a product of contributions from two fixed points at the north and the south poles of the exceptional $\mathbb{P}^{1}$, which arise due to the action of the $\mathbb{C}^{*} \times \mathbb{C}^{*}$ on $\widehat{\mathbb{P}}^{2}$ parametrized by $\epsilon_{1}$ and $\epsilon_{2}$. Upon evaluation of the Atiyah-Bott localization formula the two contributions yield - up to calculable factors - a sum of products of the original partition function on $\mathbb{P}^{2}$ at shifted $\epsilon_{1,2}$ and Coulomb branch (in type IIA normalisable Kähler) parameters. The partition function on $\widehat{\mathbb{P}}^{2}$ can be also directly specialised for $k=0$ to the one on $\mathbb{P}^{2}$. The identification of the two results gives rise to a finite set of equations for the partition function of the $4 \mathrm{~d}$ supersymmetric theory on the Omega background. A similar mechanism applies to the K-theoretic instanton calculus [16, 18], and leads to blowup equations for the partition function of the $5 \mathrm{~d}$ SYM on the Omega background. The latter setup is directly relevant to the calculation of $Z\left(\underline{t}, \epsilon_{1}, \epsilon_{2}\right)$ on non-compact Calabi-Yau engineering supersymmetric gauge theories. The blowup equations can then be reformulated in terms of the geometric data of the non-compact Calabi-Yau $X$ and refined topological string partition function as follows.

Let $C=\left(C_{i j}\right)$ be the intersection matrix between compact divisor classes $\left[\mathfrak{D}_{i}\right]$, $i=1, \ldots, b_{4}^{c}$ and compact curve classes $\left[\Sigma_{j}\right], j=1, \ldots, b_{2}^{c}$ of $X$. Then one defines vector

$$
\underline{R}_{\underline{n}}=C \cdot \underline{n}+\underline{r} / 2,
$$

with $\underline{n} \in \mathbb{Z}^{b^{c}}$ and $\underline{r} \in \mathbb{Z}^{b_{4}^{c}}$ which parametrise the shift of the Kähler parameters. With $|\underline{n}|=\sum_{i=1}^{b_{4}^{c}} n_{i}$, the generalized blowup equations can be cast in the following form [15] 
(see also section 8 of [23] and [29])

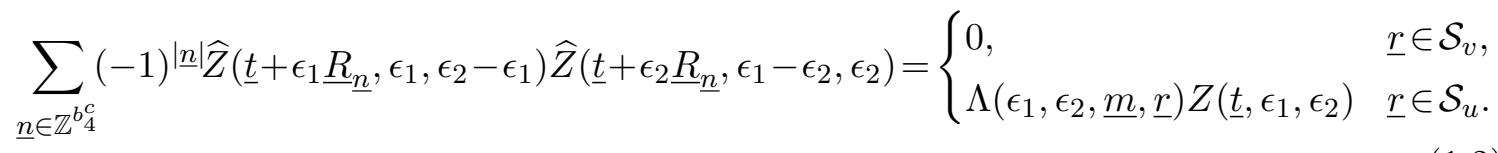

Here we have separated the Kähler parameters $\underline{m}$ from the Kähler parameters $\underline{t}$ to denote those curve classes that do not intersect with compact divisors $\left[\mathfrak{D}_{k}\right], k=1, \ldots, b_{4}^{c}$. These $\underline{m}$ correspond to mass parameters in the gauge theory context, while the other Kähler parameters correspond to Coulomb branch parameters, thus are also called "true" parameters. If a local mirror curve exists, e.g. for non-compact toric Calabi-Yau spaces, the "true" Kähler parameters are mapped to the complex structure parameters of the (hyperelliptic) mirror curve (of genus $g=b_{4}^{c}$ ) and the $\underline{m}$ correspond to the residues of the meromorphic differential $\lambda$. The hat over $Z$ means the Kähler moduli in the instanton partition function have already been shifted

$$
\widehat{Z}\left(\underline{t}, \epsilon_{1}, \epsilon_{2}\right)=Z^{\mathrm{cls}}\left(\underline{t}, \epsilon_{1}, \epsilon_{2}\right) Z^{\mathrm{inst}}\left(\underline{t}+\pi \underline{\mathrm{i}}, \epsilon_{1}, \epsilon_{2}\right) .
$$

The integral vector $\underline{r}$, which we call the $\underline{r}$-field, is consistent with the checkerboard pattern of refined BPS invariants $N_{j_{l}, j_{r}}^{\beta}$, in other words, they satisfy

$$
2 j_{l}+2 j_{r}+1 \equiv \underline{r} \cdot \beta \quad \bmod 2
$$

for non-vanishing $N_{j_{l}, j_{r}}^{\beta}$. The set of $\underline{r}$-fields in (1.2) have to be only considered modulo $2 C \cdot \underline{n}$, which leaves two classes of finite sets $\mathcal{S}_{v}$ and $\mathcal{S}_{u}$. The $\underline{r}$-fields in these two sets are called vanishing and unity $\underline{r}$-fields. It is important that $\Lambda$, whose form is known, depends beside on $\epsilon_{1,2}$ only on $\underline{r}$-fields in the two classes and the mass parameters $\underline{m}$.

Given the simple form of (1.2) and the method of proof in the gauge theory context $[16,18]$, it seems reasonable to conjecture $[15,23,25]$ that these equations, called the generalised blowup equations, should hold for the refined partition functions $Z\left(\underline{t}, \epsilon_{1}, \epsilon_{2}\right)$ of all non-compact Calabi-Yau threefolds with a global $\mathrm{U}(1)_{R}$ symmetry so that the refined invariants or equivalently the corresponding BPS index for the space time theory with an $\Omega$ background can be defined [11, 12]. At the technical level the precise non-trivial claim is that $\mathcal{S}_{v} \cup \mathcal{S}_{u}$ should be non-empty. In addition it was observed in [15] that the classical topological data of $X$ mentioned above and the genus zero sector determine $Z\left(\underline{t}, \epsilon_{1}, \epsilon_{2}\right)$ recursively, and many examples were already checked in great detail. In particular in [25] this approach was used to compute the refined BPS invariants of elliptic non-compact CalabiYau geometries associated to minimal $6 \mathrm{~d}(1,0)$ SCFTs with gauge group $G=\mathrm{SU}(3), \mathrm{SO}(8)$. In this paper we extend this approach to the remaining minimal 6d SCFTs with exceptional gauge groups $G=F_{4}, E_{6,7,8}$ and give a universal description for all minimal building blocks without matter in the classification of $6 \mathrm{~d}(1,0)$ SCFTs $[5,6]$.

The elliptic non-compact Calabi-Yau geometry corresponding to minimal SCFTs with gauge group $G$ and no matter contains base surface $\mathcal{O}(-\mathfrak{n}) \rightarrow \mathbb{P}^{1}$. For $\mathfrak{n}=3,4,5,6,8,12$ which are of interest in this paper, the Kodaire singularity of the elliptic fibration gives 
the gauge group $G=\mathrm{SU}(3), \mathrm{SO}(8), F_{4}, E_{6,7,8}$ respectively. We find that for these geometries, the generalized blowup equations can be uniformly written as the following recursive relations of the elliptic genera $\mathbb{E}_{d}$ of the corresponding $6 \mathrm{~d}$ SCFT:

$$
\begin{aligned}
\sum_{\underline{\omega} \in \phi_{\underline{\lambda}}\left(Q^{\vee}\right), d_{1,2} \in \mathbb{N}}^{\frac{1}{2}\|\underline{\omega}\|^{2}+d_{1}+d_{2}=d}( & -1)^{\left|\phi_{\underline{\lambda}}^{-1}(\underline{\omega})\right|} \theta_{i}^{[a]}\left(\mathfrak{n} \tau,(\mathfrak{n}-2)\left(\epsilon_{1}+\epsilon_{2}\right)-\mathfrak{n}\left(\left(\left.\frac{1}{2}|| \underline{\omega}\right|^{2}+d_{1}\right) \epsilon_{1}+\left(\frac{1}{2}|| \underline{\omega} \|^{2}+d_{2}\right) \epsilon_{2}-\underline{m} \cdot \underline{\omega}\right)\right) \\
& \times A_{\underline{\omega}}(\underline{m}) \mathbb{E}_{d_{1}}\left(\tau, \underline{m}-\epsilon_{1} \underline{\omega}, \epsilon_{1}, \epsilon_{2}-\epsilon_{1}\right) \mathbb{E}_{d_{2}}\left(\tau, \underline{m}-\epsilon_{2} \underline{\omega}, \epsilon_{1}-\epsilon_{2}, \epsilon_{2}\right) \\
= & \begin{cases}0, & d \notin \mathbb{N}, \\
\theta_{i}^{[a]}\left(\mathfrak{n} \tau,(\mathfrak{n}-2)\left(\epsilon_{1}+\epsilon_{2}\right)\right) \cdot \mathbb{E}_{d}\left(\tau, \underline{m}, \epsilon_{1}, \epsilon_{2}\right), & d \in \mathbb{N} .\end{cases}
\end{aligned}
$$

Here $\underline{m}$ are the Coulomb parameters ${ }^{6}$ associated to gauge group $G$. The subscript of theta functions $i$ is 4 if $\mathfrak{n}$ is odd and 3 if $\mathfrak{n}$ is even, and the characteristic $a=k / \mathfrak{n}-1 / 2$, $k=0,1, \ldots, \mathfrak{n}-1$. Besides, $\phi_{\underline{\lambda}}$ is an embedding of the coroot lattice $Q^{\vee}$ of $G$ into the weight lattice $P$. Here the $\underline{r}$-field is implicit in $a$ and $\phi_{\underline{\lambda}}$. Since the number of different embeddings is $\left|P: Q^{\vee}\right|$, the total number of non-equivalent blowup equations is $\mathfrak{n}\left|P: Q^{\vee}\right|$. The function $A_{\underline{\omega}}(\underline{m})$ is composed of $\theta_{1}$ and $\eta$ functions, see (3.5) for the definition. Due to the Jacobi form nature of every component of the above equations, we call (1.5) as elliptic blowup equations. In fact, they can be regarded as the natural elliptic lift of the K-theoretic blowup equations for 5d gauge theories [16, 30]. Moreover, the unity elliptic blowup equations in (1.5) ultimately lead to a complete solution of the elliptic genera $\mathbb{E}_{d}$ in terms of an universal recursion formula, as will be shown in (3.32). The blowup equations are not only effective tools to calculate the refined partition functions, but also together with the general constraints from modularity and BPS structure shed some new light on the structure of $Z\left(\underline{t}, \epsilon_{1}, \epsilon_{2}\right)$. In particular it is possible to derive from the structure of the blowup equations the index and the weight of the Jacobi forms that constitute the building blocks in (1.5). The recursive structure also helps clarify the form of the denominator of elliptic genus in the modular boostrap approach as discussed in appendix E, and predicts many non-trivial relations among these Jacobi forms, one particular of which is proven in section 3.3.1.

In the program of classifying superconformal field theories in various dimensions the $6 \mathrm{~d}$ SCFTs play a similar role as $11 \mathrm{~d} \mathcal{N}=1$ supergravity or more precisely M-theory play for the classification of supergravity in lower dimensions. For this reason we expect that various limits as well as suitable expansion of the partition function $Z\left(\underline{t}, \epsilon_{1}, \epsilon_{2}\right)$ of the $6 \mathrm{~d}(1,0)$ minimal SCFTs relate to the protected quantities in lower dimensional supersymmetric theories.

Since the elliptic blowup equations determine $Z\left(\underline{t}, \epsilon_{1}, \epsilon_{2}\right)$ in particular the elliptic genus $\mathbb{E}_{d}$ completely, we could make many detailed and indeed successful checks on our results. We summarise the current status of the knowledge on the elliptic genera of all $6 \mathrm{~d}(1,0)$ minimal SCFTs from various approaches in table 1 . For $\mathfrak{n}=5,6,8,12$ which are of main interest in the current paper, our complete recursive solution for the elliptic genera from blowup equations reproduces all previous partial results. Since we made many checks of

\footnotetext{
${ }^{6}$ Do not confuse with $\underline{m}$ in (1.2). The Coulomb parameters are not mass parameters.
} 


\begin{tabular}{|c|c|c|c|c|c|c|c|c|c|}
\hline$n$ & 1 & 2 & 3 & 4 & 6 & 8 & 12 & 5 & 7 \\
\hline features & E-strings & M-strings & $\mathrm{SU}(3)$ & $\mathrm{SO}(8)$ & $E_{6}$ & $E_{7}$ & $E_{8}$ & $F_{4}$ & $E_{7}+\frac{1}{2} \mathbf{5 6}$ \\
\hline $2 \mathrm{~d}$ quiver & $\mathbb{E}_{k}[32,33]$ & $\mathbb{E}_{k}[19]$ & $\mathbb{E}_{k}[34]$ & $\mathbb{E}_{k}[8]$ & \multicolumn{5}{|c|}{$?$} \\
\hline B-model & low genus [35] & $?$ & \multicolumn{7}{|c|}{ genus zero $[8]$} \\
\hline modular bootstrap & \multicolumn{2}{|c|}{$\mathbb{E}_{k}[23,36]$} & \multicolumn{2}{|c|}{$\mathbb{E}_{1}[24,37]$} & \multicolumn{5}{|c|}{$\mathbb{E}_{1}$ with fugacities off $[37,38]$} \\
\hline topological vertex & $\mathbb{E}_{k}[39]$ & $\mathbb{E}_{k}[19]$ & \multicolumn{5}{|c|}{$\mathbb{E}_{k}^{q \rightarrow 0}[40]$} & & $?$ \\
\hline Hilbert series & - & - & \multicolumn{6}{|c|}{$\mathbb{E}_{k}^{q \rightarrow 0}[41-43]$} & - \\
\hline HL index & - & - & $?$ & \multicolumn{4}{|c|}{$\mathbb{E}_{k}^{q \rightarrow 0}[44,45]$} & & - \\
\hline twisted $H_{G}$ theories & - & - & $?$ & $\mathbb{E}_{1}[37$, & $46,47]$ & $\mathbb{E}_{1}[48]$ & $?$ & & - \\
\hline domain walls & $\mathbb{E}_{1,2}[49] \mathbb{E}_{3}[50]$ & $\mathbb{E}_{k}[49]$ & \multicolumn{7}{|c|}{-} \\
\hline 5d blowup equations & \multicolumn{2}{|l|}{ trivial } & \multicolumn{6}{|c|}{$\mathbb{E}_{k}^{q \rightarrow 0}[30]$} & $\mathbb{E}_{k}^{q \rightarrow 0}[51]$ \\
\hline $6 \mathrm{~d}$ blowup equations & $\mathbb{E}_{k}[15,23,52]$ & $\mathbb{E}_{k}[52]$ & \multicolumn{2}{|c|}{$\mathbb{E}_{k}[25]$} & \multicolumn{4}{|c|}{$\mathbb{E}_{k}$, current paper } & $\mathbb{E}_{k}[51]$ \\
\hline
\end{tabular}

Table 1. Known results on the elliptic genera of $6 \mathrm{~d}$ minimal $(1,0)$ SCFTs from various approaches. Here $q=Q_{\tau}=\mathrm{e}^{2 \pi \mathrm{i} \tau}$ is the modular parameter. - means the method does not apply, and ? means possible applicable but results not yet attained. HL means Hall-Littlewood.

the elliptic blowup equations based on extensive calculations, which might yield further insights, we provide the results of these calculations on a webpage [31].

Part of these checks indicated in table 1 are quite obvious as for example the $5 \mathrm{~d}$ limit gives a good confirmation of our results. Others are highly non-trivial and indicate new exciting connections to the protected quantities in lower dimensional theories. For example one of the most important tools for the analysis of the spectra and phenomena like Seiberg duality in four dimensional SCFTs are the superconformal indices for $\mathcal{N}=1,2,4$ SCFT, which count operators in the chiral rings of these theories. These indices have in turn various limits such as Macdonald indices, Hall-Littlewood indices and Schur indices which are relatively easy to compute. As explained in section 5 the latter two occur in a quite nontrivial manner in the expansion of the elliptic genera that we can efficiently calculate. This surprising relation between elliptic genera and superconformal indices was found for the rank one $H_{G}$ theories in [37]. We will push the study on such relation for all rank two and even some rank three cases. This not only sheds light on the structure of these objects, but also allows to calculate them efficiently for example in theories with no Lagrangian description in which other methods are quite difficult to carry through.

This paper is organized as follows: in section 2, we review the geometric construction of elliptic non-compact Calabi-Yau threefolds that engineer $6 \mathrm{~d}(1,0)$ minimal SCFTs, the basic properties of the generalized blowup equations in [15], and the de-affinisation procedure which was essentially already used in [25] to obtain elliptic blowup equations for $G=\mathrm{SU}(3)$ and $\mathrm{SO}(8)$. In section 3, we discuss both unity and vanishing elliptic blowup equations in detail, and derive a universal recursion formula for the elliptic genera of all minimal SCFTs with $G=\mathrm{SU}(3), \mathrm{SO}(8), F_{4}, E_{6,7,8}$. We also prove two important properties of the elliptic blowup equations, i.e. modularity and universality. In section 4 , we explicitly show for each $G$ the one- and two-string elliptic genera computed from our universal recur- 
sion formula and also some relevant information for the blowup equations, such as triple intersection numbers and the $\underline{r}$-fields. In section 5 , we discuss a surprising relation between the elliptic genera of $6 \mathrm{~d}$ minimal SCFTs and the Hall-Littlewood indices and Schur indices of $4 \mathrm{~d} \mathcal{N}=2$ superconformal $H_{G}$ theories, as was revealed for rank one in [37]. We find analogous relation indeed exist for rank two and higher. Finally, in section 6, we discuss various possible application and future directions. In a series of appendices we explain our convention, some technical details, and collect more results on elliptic genera and refined BPS invariants too lengthy to be put in the main text.

\section{Elliptic non-compact CY 3-folds and generalised blowup equations}

The generalised blowup equations proposed in [15] (see also section 8 of [23] and [29]) generalise the K-theoretic blowup equations of Nakajima and Yoshioka $[16,18]$ for $5 \mathrm{~d}$ SYM theories to all non-compact Calabi-Yau geometries that have a $\mathrm{U}(1)_{R}$ isometry which may or may not engineer $5 \mathrm{~d}$ supersymmetric field theories. In subsection 2.1 we describe the geometric data of the non-compact elliptic Calabi-Yau threefolds associated to the minimal 6d SCFTs with pure gauge bulk theory. With this input and $b_{i}^{\text {ns }}$ calculated in subsection 2.2, the $r$ fields can be determined in subsection 2.3 and the generalised blowup equations can be expanded to extract BPS constraints that allow for solution of refined BPS invariants as in [15].

We then consider the expansion of the partition function in the base degrees and describe how to recast the generalised blowup equations, with some additional input, as functional equations of elliptic genera of the 6d SCFTs. The latter, which we call the elliptic blowup equations, will be discussed in full detail in the next section.

\subsection{Geometry of elliptic fibrations}

In this paper we are specifically interested in the non compact elliptic Calabi-Yau threefolds on which F-theory compactification yields minimal $6 \mathrm{~d}(1,0)$ SCFTs with $\mathfrak{n}=3,4,5,6,8,12$ so that the bulk theory has a pure gauge group $G$.

We discuss some generic features of these Calabi-Yau threefolds, in particular the compact curves and the compact divisors in the Calabi-Yau. The compact curves and compact divisors in these geometries are best illustrated in [53] and summarised in [25]. Let us go over them here quickly. Suppose the gauge group $G$ has $\operatorname{rank} \operatorname{rk}(G)$ and its associated Lie algebra is $\mathfrak{g}$, then there are $b_{2}^{c}=\operatorname{rk}(G)+2$ linearly independent compact curves in the Calabi-Yau threefold. ${ }^{7}$ One of them is the $-\mathfrak{n}$ curve $\Sigma_{B}$ in the base, while the remaining rk +1 curves $\Sigma_{I}(I=0,1, \ldots, \mathrm{rk})$ are $\mathbb{P}^{1} \mathrm{~s}$ resulting from resolution of the singular elliptic fiber fibered over $\Sigma_{B}$. These $r+1$ curves intersect with each other according to the affine Dynkin diagram of $\mathfrak{g}$, where each node corresponds to a rational curve of selfintersection -2 and two curves intersect with intersection number 1 if the corresponding nodes are linked. We denote the curve corresponding to the affine node by $\Sigma_{0}$, and it is

\footnotetext{
${ }^{7}$ From now on, we simply denote $\operatorname{rk}(G)$ as rk to lighten the notation.
} 


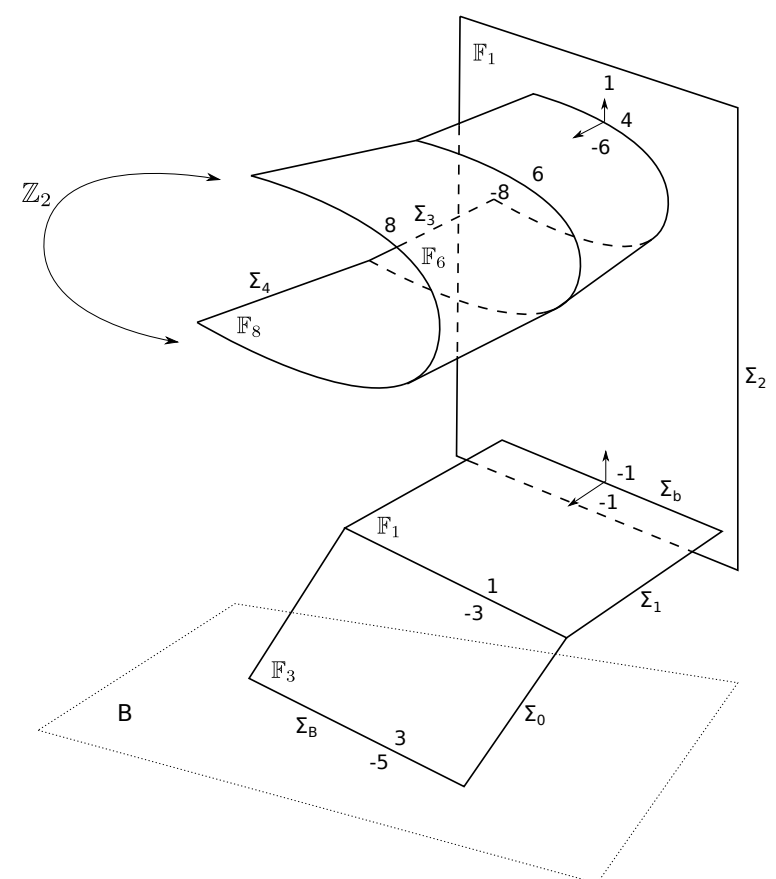

Figure 1. Compact curves and compact divisors in the $\mathfrak{n}=5$ local elliptic Calabi-Yau from [53]. The indices $(-n, n-2)$ on each rational section $S$ or $S^{\prime}=S+k F$ of $\mathbb{F}_{k}$ are the degrees of its normal bundles $\mathcal{O}(-n) \oplus \mathcal{O}(n-2)$ in the corresponding direction. $\mathbb{F}_{6}$ meets $\mathbb{F}_{1}$ in a double section. Globally the fibration has a $\mathbb{Z}_{2}$ monodromy encirceling $\Sigma_{B}$ that corresponds to an outer automorphism of the $E_{6}$ Dynkin diagram and folds its sphere tree to an $F_{4}$ type sphere tree over $\Sigma_{B}$. Note that the curve $\Sigma_{b}$ plays the role of a Mori cone generator and is related to the base curve $\Sigma_{B}$ by (4.43).

the only $\mathbb{P}^{1}$ which intersects with $\Sigma_{B}$. The linear combination

$$
\sum_{I=0}^{\mathrm{rk}} a_{I}\left[\Sigma_{I}\right]=[\delta]
$$

with $a_{I}$ the marks of $\hat{\mathfrak{g}}$ is homologous to the generic elliptic fiber. We denote the complexified Kähler parameters of $\Sigma_{B}$ and $\Sigma_{I}$ by $t_{B}$ and $t_{I}$ respectively.

The $b_{4}^{c}=\mathrm{rk}+1$ vertical compact divisors $\mathfrak{D}_{I}$ for $I=0,1, \ldots$ are fibrations of $\Sigma_{I}$ over $\Sigma_{B}$. They are argued in [53] to be Hirzebruch surfaces of various degrees, and the $\Sigma_{I}$ are the $\mathbb{P}^{1}$ fibers of these Hirzebruch surfaces. It is then easy to deduce that the $(\mathrm{rk}+2) \times(\mathrm{rk}+1)$ matrix $C$ encoding the intersections between $\Sigma_{I}, \Sigma_{B}$ and $\mathfrak{D}_{I}$ is given by

$$
C=\left(\begin{array}{c}
-\widehat{A} \\
-\mathfrak{n}, 0 \ldots, 0
\end{array}\right)
$$

where $\widehat{A}$ is the affine Cartan matrix of $G$. We illustrate compact curves and compact divisors in the example of the $\mathfrak{n}=5$ model with $G=F_{4}$ in figure 1 .

The topological string partition function on elliptic fibrations can be expanded in terms of the base degree $d$ w.r.t. the base curve $\Sigma_{B}$ labelled by $Q_{B}=\mathrm{e}^{t_{B}}$

$$
Z=Z^{\text {cls }} Z_{0}\left(1+\sum_{d=1}^{\infty} Z_{d} Q_{B}^{d}\right) \text {. }
$$


$Z^{\text {cls }}$ comes from the degree zero maps and depends hence on the classical topological data of $X$. We discuss them in section 2.2. $Z_{0}\left(t_{I}, \epsilon_{-}, \epsilon_{+}\right)$gets contributions from the rational curves $\Sigma_{I}$ in the elliptic fibre that form the affine Dynkin diagram. These can be directly calculated from the geometry reflecting the affine group structure, i.e. the intersection matrix $\hat{A}$ in $(2.2)$ using $N_{0, \frac{1}{2}}^{\left[\Sigma_{I}\right]}=1, I=0, \ldots r$ for rational curves [11] as well as the general multi cover formula (3.21), which leads to (2.36). These contribution of isolated rational curves can be also calculated as one loop correction to the gauge coupling [26], which is the reason that $Z_{0}$ is sometimes identified as $Z_{0}=Z^{1 \text {-loop }}$. The coefficients $Z_{d>0}$ in the expansion, on the other hand, encode the BPS invariants that do wrap the base curve $\Sigma_{B}$, and are rather difficult to compute.

At this point a clarification of subtlety is in order. The curve classes $\Sigma_{B}, \Sigma_{I}$ actually do not give a good basis for computing the BPS invariants, as they are not all Mori cone generators. To remedy this, one should keep $\Sigma_{I}$ and replace $\Sigma_{B}$ by the $\mathbb{P}^{1}$ base of the Hirzebruch surface with the lowest degree in the chain, so that it cannot be expressed as linear combinations of other curves with non-negative coefficients. We will illustrate this point in example section 4 .

The topological string partition function is identical with the BPS partition function of the corresponding 6d SCFT in the tensor branch, put on the Omega background $\mathbb{R}^{4} \times_{\epsilon_{1}, \epsilon_{2}}$ $T^{2}$. In the latter point of view, it is more natural to use another set of Kähler parameters $t_{\mathrm{ell}}, \tau, m_{i}(i=1, \ldots, \mathrm{rk})$, which are related to $t_{B}, t_{I}$ by

$$
t_{\mathrm{ell}}=t_{B}-\frac{\mathfrak{n}-2}{2} \tau, \quad \tau=\sum_{I=0}^{\mathrm{rk}} a_{I} t_{I}, \quad m_{i}=t_{i}, \quad i=1, \ldots, \mathrm{rk} .
$$

$t_{\text {ell }}$ is defined such that the coefficients $\mathbb{E}_{d}$ in the expansion of the BPS partition function in terms of $Q_{\text {ell }}=\mathrm{e}^{t_{\text {ell }}}$

$$
Z=Z^{\text {cls }} Z^{1-\text { loop }}\left(1+\sum_{d=1}^{\infty} \mathbb{E}_{d} Q_{\text {ell }}^{d}\right)
$$

are elliptic genera of the self-dual strings present in the 6d SCFT. $\tau$ measures the volume of the generic elliptic fiber $[\delta]$, and since $\delta$ intersect with no compact divisor, it is a mass parameter of the theory. In addition, it is also identified with the complex structure modulus of the torus $T^{2} . m_{i}$ are now interpreted as the Wilson loops of the vector multiplets in $T^{2}$.

Note that the 6d SCFT can be reduced to a $5 \mathrm{~d}$ pure SYM with the same gauge group $G$ if we decompactify $\Sigma_{0}$ and send its volume $-t_{0}$ to infinity. In the resulting $5 \mathrm{~d}$ theory, the only mass parameter is the instanton counting parameter $t_{\mathfrak{q}}$, and we find, by looking for curve class not intersecting with divisors, that

$$
t_{\mathfrak{q}}=t_{\mathrm{ell}}-\frac{\mathfrak{n}-2}{2} \tau \text {. }
$$

We finally comment that in light of the correspondence between $m_{i}$ and nodes in Dynkin diagram of $\mathfrak{g}$, we can collect the Kähler moduli $m_{i}$ into a single vector $m$ taking value in the complexified Cartan subalgebra $\mathfrak{h}_{\mathbb{C}}=\mathbb{C}^{\text {rk }}$ with

$$
m=\sum_{i=1}^{\mathrm{rk}} m_{i} \omega_{i}, \quad m_{i}=\left\langle\alpha_{i}, m\right\rangle, \quad i=1, \ldots, \mathrm{rk},
$$


where $\omega_{i}$ are the fundamental weights, $\alpha_{i}$ the simple roots of $\mathfrak{g}$, and $\langle$,$\rangle the natural pairing$ between $\mathfrak{h}_{\mathbb{C}}$ and $\mathfrak{h}_{\mathbb{C}}^{*}$. This allows a reformulation of the generalised blowup equations in terms of Lie algebraic data, which we use heavily in the uniform formula (1.5). The convention of Lie algebra we use is given in appendix A.

\subsection{Semiclassical partition functions}

We summarise the computation of the semiclassical partition functions here. These are the minimal initial data one needs in order to extract refined BPS invariants from the generalised blowup equations. First of all the semiclassical contribution $Z^{\mathrm{cls}}\left(t, \epsilon_{1}, \epsilon_{2}\right)=$ $\exp \left(F^{\mathrm{cls}}\left(t, \epsilon_{1}, \epsilon_{2}\right)\right)$ can be written as

$$
\begin{aligned}
F^{\mathrm{cls}}\left(t, \epsilon_{1}, \epsilon_{2}\right) & =\frac{1}{\epsilon_{1} \epsilon_{2}} F_{(0,0)}^{\mathrm{cls}}(t)+F_{(1,0)}^{\mathrm{cls}}(t)-\frac{\left(\epsilon_{1}+\epsilon_{2}\right)^{2}}{\epsilon_{1} \epsilon_{2}} F_{(0,1)}^{\mathrm{cls}}(t) \\
& =\frac{1}{\epsilon_{1} \epsilon_{2}}\left(\frac{1}{6} \sum_{i, j, k=1}^{b_{2}^{c}} \kappa_{i j k} t_{i} t_{j} t_{k}\right)+\sum_{i=1}^{b_{2}^{c}} b_{i}^{\mathrm{GV}} t_{i}-\frac{\left(\epsilon_{1}+\epsilon_{2}\right)^{2}}{\left(\epsilon_{1} \epsilon_{2}\right)} \sum_{i=1}^{b_{2}^{c}} b_{i}^{\mathrm{NS}} t_{i} .
\end{aligned}
$$

The coefficients $\kappa_{i j k}$ are the triple intersection numbers of divisors $J_{i}$ Poincaré dual to the curve classes $\Sigma_{i}$ with volumes $t_{i} . b_{i}^{\mathrm{GV}}$ are intersections of the divisors $J_{i}$ with the second Chern class of the Calabi-Yau threefold. The coefficients $b_{i}^{\mathrm{NS}}$, on the other hand, do not have a geometric meaning, they are usually computed by the refined holomorphic anomaly equations $[14,54]$, which are difficult to apply here. The Nekrasov partition function of a $5 \mathrm{~d}$ pure SYM also has a semiclassical contribution which takes the same form as (2.8), and the linear coefficients are subject to the relation

$$
b_{i}^{\mathrm{GV}}+b_{i}^{\mathrm{NS}}=0 .
$$

In our previous paper [25] we argued that $b_{i}^{\mathrm{GV}}, b_{i}^{\mathrm{NS}}$ of the minimal $6 \mathrm{~d}$ SCFTs with $G=$ $\mathrm{SU}(3), \mathrm{SO}(8)$ can be computed by uplifting the semiclassical Nekrasov partition function of the $5 \mathrm{~d}$ pure SYM with the same gauge $G$ aided by the nontrivial automorphism of the affine Dynkin diagrams, and we found these coefficients also satisfy the relation (2.9). In the remaining minimal SCFTs with $G=F_{4}, E_{6}, E_{7}, E_{8}$, not all the affine Dynkin diagams have a non-trivial automorphism, and the method of uplifting does not always work. Instead we assume (2.9) to be true and only compute $b_{i}^{\mathrm{GV}}$ by geometric means. ${ }^{8}$

To compute $\kappa_{i j k}$ and $b_{i}^{\mathrm{GV}}$, we need to embed the Calabi-Yau threefold $X \rightarrow \mathcal{O}_{\mathbb{P}^{1}}(-\mathfrak{n})$ in a compact Calabi-Yau, for instance the elliptic fibration $\hat{X}$ over $\mathbb{F}_{\mathfrak{n}}$ with a single section, and first compute these intersection numbers in the compact geomtry. The compact CalabiYau $\hat{X}$ can be realised as a hypersurface in a toric variety [8]. Let us look at an example in detail. The threefold $\pi: X \rightarrow \mathcal{O}_{\mathbb{P}^{1}}(-5)$ is the zero loci of the section of the anti-canonical bundle of the toric variety $\mathbb{P}_{\Delta}$, whose toric data is given in table $2[8] .{ }^{9}$ It has 7 Mori cone generators with charges $l^{(i)}=\left(l_{n}^{(i)}\right)$ for $i=1, \ldots, 7$ (We will use the same notation

\footnotetext{
${ }^{8} \mathrm{Up}$ to a irrelevant $\tau$ term, the numbers $b_{i}^{\mathrm{GV}}, b_{i}^{\mathrm{NS}}$ can be also predicted from blowup equations by requiring the consistency of BPS invariants. For all the minimal SCFTs, these predictions agree with the values computed from the method we will describe later.

${ }^{9}$ See also the geometric description in [55].
} 


\begin{tabular}{|rrrrr|rrrrrrr|rrrrrr|}
\hline$D$ & \multicolumn{1}{c}{$\nu_{i}^{*}$} & & $l^{(1)}$ & $l^{(2)}$ & $l^{(3)}$ & $l^{(4)}$ & $l^{(5)}$ & $l^{(6)}$ & $l^{(7)}$ & $l_{F_{4}}^{(0)}$ & $l_{F_{4}}^{(1)}$ & $l_{F_{4}}^{(2)}$ & $l_{F_{4}}^{(3)}$ & $l_{F_{4}}^{(4)}$ & $l_{F_{4}}^{(b)}$ \\
$D_{0}$ & 0 & 0 & 0 & 0 & -2 & 0 & 0 & 0 & 0 & 0 & 0 & 0 & 0 & 0 & 0 & -2 & 0 \\
$D_{1}$ & -1 & 0 & 0 & 0 & 0 & -2 & 0 & 0 & 0 & 0 & 1 & 1 & 0 & 0 & 0 & 0 & 0 \\
$D_{2}$ & 0 & -1 & 0 & 0 & 1 & 0 & 0 & 0 & 0 & 0 & 0 & 0 & 0 & 0 & 0 & 1 & 0 \\
$D_{3}$ & 0 & 1 & 0 & -1 & 0 & 3 & 0 & 0 & 0 & 1 & -2 & -2 & 1 & 0 & 0 & 0 & 0 \\
$D_{4}$ & 1 & 2 & 0 & -2 & 2 & 0 & 0 & 0 & 0 & -2 & 1 & 1 & -2 & 0 & 0 & 2 & 0 \\
$S^{\prime}$ & 2 & 3 & 0 & -1 & 0 & 1 & -2 & 0 & 1 & 0 & 0 & 0 & 0 & -2 & 1 & 0 & 0 \\
$S^{\prime \prime}$ & 2 & 3 & 0 & -2 & 1 & -2 & 1 & -1 & 0 & 0 & 0 & 0 & 0 & 1 & -2 & 1 & -1 \\
$S^{\prime \prime \prime}$ & 2 & 3 & 0 & -3 & -2 & 0 & 0 & -1 & 0 & 1 & 0 & 0 & 1 & 0 & 1 & -2 & -1 \\
$K$ & 2 & 3 & 0 & 0 & 0 & 0 & 1 & 0 & -2 & 0 & 0 & 0 & 0 & 1 & 0 & 0 & 0 \\
$F$ & 2 & 3 & -1 & -5 & 0 & 0 & 0 & 1 & 0 & 0 & 0 & 0 & 0 & 0 & 0 & 0 & 1 \\
$S$ & 2 & 3 & 0 & 1 & 0 & 0 & 0 & 0 & 1 & 0 & 0 & 0 & 0 & 0 & 0 & 0 & 0 \\
$F$ & 2 & 3 & 1 & 0 & 0 & 0 & 0 & 1 & 0 & 0 & 0 & 0 & 0 & 0 & 0 & 0 & 1 \\
\hline
\end{tabular}

Table 2. The toric data of $\mathbb{P}_{\Delta}$ for $G=F_{4}$.

for both the curves and their toric charges). Note the number of Mori cone generators is $(\mathrm{rk}+3)$. Among these curves $l^{(4)}, l^{(5)}$ are identified as the $(-5)$ curve (i.e. $\Sigma_{B}$ ) and the (0) curve in the base $\mathbb{F}_{5},{ }^{10}$ while the other five toric curves combine linearly into $\Sigma_{I}$ for $I=0,1, \ldots, 4[8]$

$$
\Sigma_{0}=l^{(3)}, \Sigma_{1}=l^{(2)}+l^{(6)}+2 l^{(7)}, \Sigma_{2}=l^{(1)}, \Sigma_{3}=l^{(6)}, \Sigma_{4}=l^{(7)} .
$$

The toric divisors listed in the first column of table 2 can also be identified. $D_{0}$ is associated to the canonical bundle of $\mathbb{P}_{\Delta}$. When $\hat{X}$ is written in the Weierstrass form, $D_{1}, D_{2}$ correspond to the divisors $x=0$ and $y=0$ respectively, while $K$ is the zero section at $x \rightarrow \infty$. F, $S^{\prime}, S$ are respectively the vertical divisors pulled back from the $(0),(-5)$, and the (5) curves in $\mathbb{F}_{5}$ in the base. Since the elliptic fibration over $\pi\left(S^{\prime}\right)=\Sigma_{B}$ factorises to an intersecting tree of Hirzebruch surfaces $\mathfrak{D}_{I}$ for $I=0,1, \ldots, 4, S^{\prime}$ should actually be identified with $\mathfrak{D}_{0}$. The remaining four divisors $D_{3}, D_{4}, S^{\prime \prime}, S^{\prime \prime \prime}$ are identified (up to linear combination) as the exceptional divisors $\mathfrak{D}_{i}$ for $i=1, \ldots, 4$. Only $\operatorname{rk}\left(F_{4}\right)+3=7$ of these divisors are linearly independent.

Once the toric data of $\mathbb{P}_{\Delta}$ are specified, there are standard techniques in toric geometry to compute the triple intersection numbers $\hat{\kappa}_{i j k}$ of the divisors $\hat{J}_{i}$, and the intersection numbers with $c_{2}(\hat{X})$ are given by $[56]^{11}$

$$
-24 \hat{b}_{i}^{\mathrm{GV}}=\int_{\hat{X}} c_{2}(\hat{X}) \wedge \hat{J}_{i}=\frac{1}{2} \sum_{j k} \hat{\kappa}_{i j k}\left(l_{0}^{(j)} l_{0}^{(k)}-\sum_{n>0} l_{n}^{(j)} l_{n}^{(k)}\right) .
$$

\footnotetext{
${ }^{10}$ Strictly speaking, the (0) curve should be corrected by certain linear combination of other curves. But after decompactifying this curve to arrive at the non-compact threefold $X$, this difference disappears.

${ }^{11} \mathrm{We}$ use the same notation for the divisor $\hat{J}_{i}$ and its dual 2 -form.
} 
Alternatively, $\hat{\kappa}_{i j k}$ can be computed by the special geometry relation. For a compact Calabi-Yau threefold realised as a hypersurface in a toric variety one can define the deformed fundamental period $\hat{\omega}_{0}(z ; \rho)$ as a holomorphic function of the Batyrev coordinates $z_{i}$ and compute the homogeneous A- and B-periods (see for instance [57])

$$
\begin{aligned}
\hat{\Pi}_{i}^{(1)}(z) & =\left.\partial_{\rho_{i}} \hat{\omega}_{0}(z ; \rho)\right|_{\rho_{i}=0}, \\
\hat{\Pi}_{i}^{(2)}(z) & =\left.\frac{1}{2} \hat{\kappa}_{i j k} \partial_{\rho_{j}} \partial_{\rho_{k}} \hat{\omega}_{0}(z ; \rho)\right|_{\rho_{i}=0} .
\end{aligned}
$$

They are interpreted as the masses of the D2-, D4-branes supported on the curves $l^{(i)}$ and the dual divisors $\hat{J}_{i}$. The affine A- and B-periods defined by

$$
\hat{t}_{i}=\hat{\Pi}_{i}^{(1)} / \hat{\omega}_{0}(z ; 0), \hat{F}_{i}=\hat{\Pi}_{i}^{(2)} / \hat{\omega}_{0}(z ; 0)
$$

satisfy the relation

$$
\hat{F}_{i}=\frac{\partial \hat{F}_{(0,0)}}{\partial \hat{t}_{i}} .
$$

The existence of the prepotential $\hat{F}_{(0,0)}$ uniquely fixes the coefficients $\hat{\kappa}_{i j k}$.

The non-compact Calabi-Yau $X$ is obtained by decompactifying $\hat{X}$ in the direction of the (0)-curve in the base. In practise, this corresponds to taking the Kähler parameter in the decompactified direction $t_{\mathrm{dc}}$ to infinity in A-model or taking the corresponding complex structure parameter $z_{\mathrm{dc}}$ to zero in B-model. In A-model, this limit can be understood as taking some of the compact $(1,1)$ cycles to infinite size, keeping the other $(1,1)$ cycles finite. The periods of the geometry will be rearranged so that only one A-period and some B-periods go to infinity. In our current case, the B-period $\frac{\partial}{\partial \tau} F_{(0,0)}$ goes to infinity, while the corresponding A-period remains finite, and becomes the elliptic fiber parameter $\tau$. We can then integrate over the new periods to get the triple intersection numbers of the non-compact geometry. However, this method will always have a integration constant term $\tau^{3}$ unfixed, which is very important for the refined BPS invariants in the $\tau$ direction. To determine the $\tau^{3}$ term, we study the normalization scheme of $\tau$ derivative of the genus zero free energy $\frac{\partial}{\partial \tau} F_{(0,0)}$.

In the example of the $G=F_{4}$ model visited above, this is $z_{5}$ associated to the curve $l^{(5)}$. In the limit $z_{\mathrm{dc}} \rightarrow 0$ the affine A-period associated to the (0)-curve diverges, while the other $\mathrm{rk}+2$ affine A-periods remain finite. We can choose a basis of the latter to be ${ }^{12}$

$$
\left(t_{I}\right), \quad I=0,1, \ldots, \mathrm{rk}, B
$$

which correspond to the curve classes $\Sigma_{0}, \ldots, \Sigma_{r}, \Sigma_{B}$ discussed in the previous section. At the same time, both the zero section of the elliptic fibration and the vertical disivor of the (0)-curve become infinite in volume. Therefore in the limt $z_{\mathrm{dc}} \rightarrow 0$ two B-periods diverge, and only $\mathrm{rk}+1$ affine B-periods remain finite. We choose a basis

$$
\left(F_{J}\right), \quad J=0,1, \ldots, \mathrm{rk},
$$

\footnotetext{
${ }^{12}$ They are the limit $z_{\mathrm{dc}} \rightarrow 0$ of proper linear combinations of $\hat{t}_{i}$ of the compact Calabi-Yau. Similarly $F_{J}$ defined below are the limit $z_{\mathrm{dc}} \rightarrow 0$ of linear combinations of $\hat{F}_{i}$.
} 
which correspond to the divisor classes $\mathfrak{D}_{I}$ with $I=0,1, \ldots$, rk. The special geometry relation of the non-compact Calabi-Yau $X$ dictates

$$
F_{J}=\sum_{I=0, \ldots, \mathrm{rk}, B} C_{J I} \frac{\partial F_{(0,0)}}{\partial t_{I}}, \quad J=0,1, \ldots, \mathrm{rk},
$$

where $C_{J I}$ are the components of the divisor-curve intersection matrix (2.2). The identity (2.17) allows the computation of the semi-classical components of $F_{(0,0)}(t)$ up to a term proportional to $\tau^{3}$; in other words, the intersection number $\kappa_{\tau \tau \tau}$ can not be fixed by (2.17). Since $\tau$ is a mass parameter, the term $\tau^{3}$ can always be factored out of the blowup equations and it is not of importance to us. Nevertheless, in appendix B we will introduce a normalisation scheme which fixes such a term in a reasonable way, and we adopt this normalisation scheme in the example section 4. In any case, for all the minimal $6 \mathrm{~d}$ SCFTs with a pure gauge theory in the bulk which can be reduced to a $5 \mathrm{~d}$ pure SYM, we find that up to $\tau^{3}$

$$
F_{(0,0)}^{\mathrm{cls}}(t)=-\sum_{\alpha \in \Delta_{+}}\left(\frac{\langle\alpha, m\rangle^{3}}{6}+\frac{t_{\mathfrak{q}}}{2 h_{G}^{\vee}}\langle\alpha, m\rangle^{2}\right)-\frac{\left((\mathfrak{n}-2) \tau+t_{\mathfrak{q}}\right)^{3}-t_{\mathfrak{q}}^{3}}{6 \mathfrak{n}(\mathfrak{n}-2)}+\ldots
$$

where $m, t_{\mathfrak{q}}$ are defined in (2.7) and (2.6) respectively. We recognise the sum over positive roots is from the Nekrasov prepotenital of the 5d pure SYM. We can also massage (2.18) into a more suggestive form

$$
F_{(0,0)}^{\mathrm{cls}}=\frac{1}{2 \mathfrak{n}} t_{\mathrm{ell}}^{2} \tau+\frac{1}{2} t_{\mathrm{ell}}(m, m)-\frac{\mathfrak{n}-2}{4} \tau(m, m)+\ldots
$$

up to $\tau^{3}$ and cubic terms in $m_{i}$. Here $($,$) is the invariant bilinear form on \mathfrak{h}_{\mathbb{C}}$. See appendix A for our convention.

As for $F_{(1,0)}^{\text {cls }}(t)$ and the intersection numbers with $c_{2}(X)$, we use the same formula (2.11) with $\hat{\kappa}_{i j k}$ replaced by the triple intersection numbers of the non-compact Calabi-Yau and $l^{(i)}$ replaced by the toric charges of $\Sigma_{0}, \ldots, \Sigma_{r}, \Sigma_{B}$. In the example of $G=F_{4}$ discussed above, one has the toric charges $l_{F_{4}}^{(0)}, l_{F_{4}}^{(1)}, l_{F_{4}}^{(2)}, l_{F_{4}}^{(3)}, l_{F_{4}}^{(4)}, l_{F_{4}}^{(b)}$ as in table 2 .

As in the case of the prepotential, one cannot determine the pure mass term proportional to $\tau$ which is irrelevant, although it can be fixed by the same normalisation scheme if one wishes. We have checked that $F_{(1,0)}^{\text {cls }}(t)$ computed in this way reduces correctly to the semiclassical Nekrasov partition function when the $6 \mathrm{~d}$ SCFT is reduced to the $5 \mathrm{~d}$ pure SYM.

\subsection{Determination of $r$ fields}

In general, the $r$ fields associated to a non-compact Calabi-Yau can be determined by the method in [15]. Here we give a brief description of it. As proved in [15], even without any assumption or constraint put on $\Lambda$ (for instance it can depend on all Kähler moduli), the $\Lambda$ as defined by (1.2) must be quasi-modular of weight zero under the Siegel modular transformations of $\tau_{i j}=\frac{\partial^{2}}{\partial t_{i} \partial t_{j}} F_{(0,0)}$, where $t_{i}$ are the true Kähler parameters. ${ }^{13}$ Let us

\footnotetext{
${ }^{13}$ Note that this $\tau_{i j}$ parameter is different from the $\tau$ parameter of the elliptic fiber.
} 
expand this $\Lambda$ in terms of all the exponentiated Kähler moduli $\mathrm{e}^{t_{i}}$. The leading terms, which come from $Z^{\text {cls }}$, read

$$
\begin{aligned}
\log \Lambda & \sim \log \left(Z^{\mathrm{cls}}\left(\epsilon_{1}, \epsilon_{2}-\epsilon_{1}\right) Z^{\mathrm{cls}}\left(\epsilon_{1}-\epsilon_{2}, \epsilon_{2}\right) / Z^{\mathrm{cls}}\left(\epsilon_{1}, \epsilon_{2}\right)\right) \\
& =\left(-\frac{1}{6} \sum_{i, j, k=1}^{b_{2}^{c}} \kappa_{i j k} R_{i} R_{j} R_{k}+\sum_{i=1}^{b_{2}^{c}}\left(b_{i}^{\mathrm{GV}}-b_{i}^{\mathrm{NS}}\right) R_{i}\right)\left(\epsilon_{1}+\epsilon_{2}\right)+\sum_{k=1}^{b_{2}^{c}}\left(-\frac{1}{2} \sum_{i, j=1}^{b_{2}^{c}} \kappa_{i j k} R_{i} R_{j}\right) t_{k} \\
& =: f_{0}(\underline{n})\left(\epsilon_{1}+\epsilon_{2}\right)+\sum_{k=1}^{b_{2}^{c}} f_{k}(\underline{n}) t_{k},
\end{aligned}
$$

which are linear in $t_{i}$. It implies that $\Lambda$ can be expanded as a well-defined power series in $e^{t_{i}}$. Now let us assume that for appropriate choice of the $r$-field, this power series with all the instanton contributions taken into account truncates at finite orders for all true $t_{i}{ }^{14}$ which actually implies that the $\Lambda$ does not depend on any of the true Kähler parameters as stated in the introduction, since otherwise it can not be of modular weight zero. Needless to say, this assumption puts very strong constraint on the choice of the $r$-field, and these are the $r$-fields we are interested in.

In our current cases of elliptic non-compact Calabi-Yau threefolds, there is only one mass parameter which is $\tau$. The strong constraint then means that for the $r$ fields we are interested in only the lowest order of Kähler parameters contributes. Then we can simply define $\Lambda$

$$
\Lambda\left(\tau, \epsilon_{1}, \epsilon_{2}\right)=\sum_{\underline{n} \in \mathcal{I}}(-1)^{|\underline{n}|} \mathrm{e}^{f_{0}(\underline{n})} \mathrm{e}^{f_{k}(\underline{n}) t_{k}},
$$

where $\mathcal{I}$ is the set of integral vectors $\underline{n}$ that minimize all the $f_{k}(\underline{n})$ for true Kähler parameters simultaneously after subtracting mass parameters. If the minimal values for one $r$ are not zero simultaneously, then it must be a vanishing $r$ or an incorrect $r$.

In the case of elliptic non-compact Calabi-Yau threefolds associated to minimal $6 \mathrm{~d}$ SCFTs, the $\tau$ parameter is always some combinations of Kähler parameters in the fiber direction and it will be a little bit subtle to subtract the mass parameter $\tau$. We can first consider the $\tau$ irrelevant Kähler parameter $t_{\text {ell }}$, and the minimum of the associated $f_{\text {ell }}(\underline{n})$, and then check the solved $r$ fields with the condition (2.21). As also shown in [25], the existence of the minimum of $f_{\text {ell }}(\underline{n})$, already suffices to fix all the $r$ fields. In particular, similar to $t$, we decompose $r$ into components $r_{0}, r_{1}, \ldots, r_{\mathrm{rk}}, r_{B}$. Then the weak consistency condition implies the admissibility condition, which we will prove shortly

$$
r_{\tau}=\sum_{I=0}^{\mathrm{rk}} a_{I} r_{I}=0 .
$$

It means the component of $r$ in the direction of the elliptic fiber must vanish. Recall that we only consider $r$ modulo $2 C \cdot \underline{n}$ for $\forall \underline{n} \in \mathbb{Z}^{b_{4}^{c}}=\mathbb{Z}^{\text {rk+1 }}$. The intersection matrix $C$ defines the injection $\mathbb{Z}^{\text {rk+1 }} \hookrightarrow \mathbb{Z}^{\text {rk+2 }}$. The $r$ fields that satisfy (2.22) can only take value in the

\footnotetext{
${ }^{14}$ This assumption is the most natural consideration for the generalized blowup equations compared with the initial Nakajima-Yoshioka blowup equations.
} 


\begin{tabular}{|ccccccc|}
\hline $\mathfrak{n}$ & 3 & 4 & 5 & 6 & 8 & 12 \\
\hline$\#(r)$ & 9 & 16 & 5 & 18 & 16 & 12 \\
$\left|P: Q^{\vee}\right|$ & 3 & 4 & 1 & 3 & 2 & 1 \\
\hline
\end{tabular}

Table 3. Numbers and sizes of groups of $r$-fields giving rise to the same embedding $\phi_{\lambda}$.

dimension zero quotient lattice $\Gamma / \mathbb{Z}^{\mathrm{rk}+1}$ with $\Gamma=\left.\mathbb{Z}^{\mathrm{rk}+2}\right|_{r_{\tau}=0}$, and they are thus finite in number. Finally we impose the BPS checkerboard pattern condition

$$
2 j_{l}+2 j_{r}+1 \equiv r \cdot \beta \quad \bmod 2
$$

to remove half of them. We comment that the checkerboard condition can be written down without computing any BPS invariants. As argued in [25], any rational curve $\beta$ in the Calabi-Yau with normal bundle $\mathcal{O}(-n) \oplus \mathcal{O}(n-2)$ must have

$$
2 j_{l}+2 j_{r}+1 \equiv n \quad \bmod 2 .
$$

This implies the $r$-field always satisfies

$$
r \equiv(0, \ldots, 0, \mathfrak{n}) \quad \bmod 2,
$$

for minimal $6 \mathrm{~d}(1,0)$ SCFTs. The argument above not only establishes the finiteness of admissible $r$, but also provides a guideline on how to determine them. We find all of them for the $G=\mathrm{SU}(3), \mathrm{SO}(8)$ models in [25] and for the remaining models with $G=F_{4}, E_{6,7,8}$ in the example section 4 of this paper. In all these examples we checked that they satisfy the stronger consistency condition. Here we summarise their numbers in table 3 .

Let us prove the admissibility condition (2.22). An important ingredient of the blowup equations (1.2) are the shifts of Kähler moduli $m_{i}(i=1, \ldots$, rk $)$ by

$$
R_{i}=\sum_{J=0}^{\mathrm{rk}} C_{i, J} n_{J}+\frac{1}{2} r_{i}
$$

where $n_{J}$ take value in $\mathbb{Z}$. We collect them into a single vector just like $m$

$$
R=\sum_{i=1}^{\mathrm{rk}}\left(-\sum_{J=0}^{\mathrm{rk}} \hat{A}_{i, J} n_{J}+\frac{1}{2} r_{i}\right) \omega_{i}=-\alpha^{\vee}+\lambda+n_{0} \theta,
$$

where we have used the generic form (2.2) of the intersection matrix $C$ and the following Lie algebraic notation

$$
\lambda=\frac{1}{2} \sum_{i=1}^{\mathrm{rk}} r_{i} \omega_{i}, \quad \alpha^{\vee}=\sum_{i=1}^{\mathrm{rk}} n_{i} \alpha_{i}^{\vee} .
$$

The function $f_{\text {ell }}(\underline{n})$, which we also denote by $P_{G}(\underline{n})$ due to its particular importance, then has the form

$$
f_{\mathrm{ell}}(\underline{n})=\frac{1}{\mathfrak{n}} R_{\mathrm{ell}} R_{\tau}+\frac{1}{2}(R, R)=: P_{G}(\underline{n}),
$$


where $R_{\text {ell }}, R_{\tau}$ are the shifts of $t_{\text {eff }}, \tau$ given respectively by

$$
R_{\mathrm{ell}}=-\mathfrak{n} n_{0}+\frac{1}{2} r_{\mathrm{ell}}, \quad R_{\tau}=\frac{1}{2} r_{\tau}=\frac{1}{2} \sum_{I=0}^{\mathrm{rk}} a_{I} r_{I} .
$$

A little algebraic manipulation leads to

$$
P_{G}(\underline{n})=\frac{1}{2} \sum_{I, J=0}^{\mathrm{rk}} n_{I} n_{J}\left(\alpha_{I}^{\vee}, \alpha_{J}^{\vee}\right)-\frac{1}{2} \sum_{I, J=0}^{\mathrm{rk}} r_{I} n_{J}\left(\widehat{\omega}_{I}, \alpha_{J}^{\vee}\right)+\ldots
$$

where ... denote the terms that are independent from $\underline{n}$. If we demand that this function have a minimum, all the derivatives $\partial_{n_{I}} P_{G}(\underline{n})$ must have a common zero. Multiplying each of them with comark $a_{I}$ and adding them up, we immediately arrive at the admissibility condition (2.22). ${ }^{15}$ Let us make some remarks here. As we will see in section 4, all the admissible $r$-fields are such that the components $r_{i}(i=1, \ldots, \mathrm{rk})$ are even integers. It is clear then that $\lambda$ associated to $r$ as well as the shift $R$ have nice interpretation as weight vectors of $\mathfrak{g}$. And $P_{G}(\underline{n})$ is nothing else but half the norm square of $R$ due to (2.22)

$$
P_{G}(\underline{n})=\frac{1}{2}(R, R) .
$$

Furthermore $\lambda$ defines an embedding of $Q^{\vee}$ into $P$

$$
\begin{aligned}
\phi_{\lambda}: Q^{\vee} & \hookrightarrow P \\
\alpha^{\vee} & \mapsto \phi_{\lambda}\left(\alpha^{\vee}\right)=-\alpha^{\vee}+\lambda
\end{aligned}
$$

and $R$ takes value in the image $\phi_{\lambda}\left(Q^{\vee}\right)$ if $n_{0}=0$. The number of inequivalent embeddings is the index $\left|P: Q^{\vee}\right|$, which also happens to be the order of the automorphism group of the associated Dynkin diagram. We also list these numbers in table 3. The reader may notice the curious relation

$$
\mathfrak{n} \cdot\left|P: Q^{\vee}\right|=\#(r),
$$

whose meaning will be clear in section 3 .

\subsection{De-affinisation}

Once the semiclassical piece $Z^{\text {cls }}$ and the $r$-fields are known, we can start solving refined BPS invariants by expanding the blowup equations (1.2) in terms of Kähler moduli and extracting constraint equations of BPS invariants at each order. Alternatively since the $Z^{1 \text {-loop }}$ piece is rather easy to compute for the minimal $6 \mathrm{~d}$ SCFTs, which reads $[25]^{16}$

$$
Z^{1 \text {-loop }}\left(m, \epsilon_{1}, \epsilon_{2}\right)=\mathrm{PE}\left[-\frac{q_{R}+q_{R}^{-1}}{\left(q_{1}^{1 / 2}-q_{1}^{-1 / 2}\right)\left(q_{2}^{1 / 2}-q_{2}^{-1 / 2}\right)} \sum_{\alpha \in \Delta_{+}}\left(\mathrm{e}^{\langle\alpha, m\rangle}+Q_{\tau} \mathrm{e}^{-\langle\alpha, m\rangle}\right) \frac{1}{1-Q_{\tau}}\right],
$$

\footnotetext{
${ }^{15}$ This condition was found in [25] by an intuitive geometric argument for minimal theories with a gauge group $G$ of the ADE type. Here we prove it for all gauge groups including the non-ADE types.

${ }^{16}$ Here PE is the plethystic exponent operator defined as

$$
\mathrm{PE}[f(x)]=\exp \left[\sum_{n=1}^{\infty} \frac{1}{n} f\left(x^{n}\right)\right] .
$$

Note we only consider the contribution from vector multiplets to the one-loop partition function here. The tensor multiplets actually also contribute to one-loop partition function, but their contribution does not depend on the gauge parameter $m$, i.e. it is pure $\tau$ terms which will decouple from the blowup equations. Thus we do not consider them here. See more discussion in section 2.4 in [25].
} 
we can also plug in this piece of information, expand the partition functions in the blowup equation (1.2) only in terms of $Q_{\text {ell }}$, and obtain recursion relations of elliptic genera

$$
\begin{gathered}
\sum_{P_{G}(\underline{n})+d_{1}+d_{2}=d} D^{\prime, \text { cls }} D^{1 \text {-loop }} \\
\mathbb{E}_{d_{1}}\left(\tau, m+\epsilon_{1} R_{m}(\underline{n}), \epsilon_{1}, \epsilon_{2}-\epsilon_{1}\right) \mathbb{E}_{d_{2}}\left(\tau, m+\epsilon_{2} R_{m}(\underline{n}), \epsilon_{1}-\epsilon_{2}, \epsilon_{2}\right) \\
=\Lambda \mathbb{E}_{d}\left(\tau, m, \epsilon_{1}, \epsilon_{2}\right)
\end{gathered}
$$

which allows the solution of the elliptic genera in compact formulas. Here

$$
D^{\prime, \text { cls }}=(-1)^{|\underline{n}|+\left(d_{1}+d_{2}-d\right) r_{\text {ell }}} \exp \left(f_{0}(\underline{n})\left(\epsilon_{1}+\epsilon_{2}\right)+\sum_{I=0}^{\mathrm{rk}} f_{I}(\underline{n}) t_{I}+\left(d_{1} \epsilon_{1}+d_{2} \epsilon_{2}\right) R_{\mathrm{ell}}(\underline{n})\right)
$$

collects contributions from the semiclassical partition function (as well as the shift of $t_{\text {ell }}$ ), and

$$
D^{1 \text {-loop }}=Z^{1 \text {-loop }}\left(\tau, m+\epsilon_{1} R_{m}(\underline{n}), \epsilon_{1}, \epsilon_{2}-\epsilon_{1}\right) Z^{1 \text {-loop }}\left(\tau, m+\epsilon_{2} R_{m}(\underline{n}), \epsilon_{1}-\epsilon_{2}, \epsilon_{2}\right) / Z^{1 \text { loop }}\left(\tau, m, \epsilon_{1}, \epsilon_{2}\right)
$$

is the contribution from the one-loop partition function. The elliptic blowup equations can be put in an elegant form by partially resumming the left hand side of (2.37). With (2.31) and the admissibility condition (2.22), one can show that the polynomial $P_{G}(\underline{n})$ that characterises the summation index $\underline{n}$ is invariant under the translation

$$
n_{I} \rightarrow n_{I}+a_{I}^{\vee} k, \quad k \in \mathbb{Z} .
$$

Besides, in the components $Z^{1 \text {-loop }}$ and $\mathbb{E}_{d}$ the dependence on $\underline{n}$ only appears in the shifts

$$
R_{I}=-\sum_{J=0}^{\mathrm{rk}} \hat{A}_{I, J} n_{J}+\frac{1}{2} r_{I}, \quad I=0,1, \ldots, \mathrm{rk},
$$

which are also invariant under (2.40). As a result, we can decompose the summation index

$$
\underline{n}=\underline{\hat{n}}+a^{\vee} k
$$

with the zeroth component of $\underline{\hat{n}}$ fixed to zero, i.e. $\underline{\hat{n}}=\left(0, n_{1}, n_{2}, \ldots, n_{\mathrm{rk}}\right)$, a step we call "de-affinisation", and perform the infinite sum on the left hand side of (2.37) in two steps.

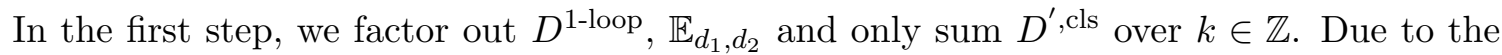
quadratic nature of the polynomials $f_{0}(\underline{n}), f_{I}(\underline{n})$, and the relation of $t_{I}$ with $\tau(2.4)$, this first summation in fact produces a theta function with characteristics [25]. In the second step, we sum over $d_{1}, d_{2}$ and $\underline{\hat{n}}$ that satisfy

$$
P_{G}(\underline{\hat{n}})+d_{1}+d_{2}=d .
$$

Instead of $\underline{\hat{n}}$ we can treat $R(\underline{\hat{n}})$ as the summation index, which as we argued before is now interpreted as a weight vector in $\phi_{\lambda}\left(Q^{\vee}\right)$ determined by $r$. In addition, the oneloop contribution $D^{1 \text {-loop }}$ also turns out to be a quotient of theta functions [25], while the elliptic genera themselves are meromorphic Jacobi forms. Therefore in the end, the elliptic blowup equations can be presented as beautiful equations of Jacobi forms with a sum over the shifted coroot lattice $\phi_{\lambda}\left(Q^{\vee}\right)$. These equations are the highlights of the next section. We will present these equations in the beginning of the next section, and then discuss their properties and how to solve elliptic genera from them. 


\section{Elliptic blowup equations}

In this section, we first present the elliptic blowup equations for $6 \mathrm{~d}$ minimal $\mathcal{N}=(1,0)$ SCFTs with $G=\mathrm{SU}(3), \mathrm{SO}(8), F_{4}, E_{6}, E_{7}, E_{8}$, and discuss their two interesting properties, the modularity and the universality. The first property in particular serves as a strong support for the validity of the elliptic blowup equations to arbitrary degrees. Then we distinguish two cases with $\Lambda\left(\epsilon_{1}, \epsilon_{2}, m, r\right)$ non-vanishing or identically vanishing, and discuss these two cases in detail. In particular, the blowup equations in the first case with nonvanishing $\Lambda$ allow us to write down an exact and universal recursion formula for elliptic genera, thus offering a complete solution to the elliptic genera.

Let us first fix some conventions. In the following whenever there is no risk of confusion we will use the dot to denote both the invariant bilinear form on $\mathfrak{h}$ or $\mathfrak{h}^{*}$ and the natural inner product between $\mathfrak{h}$ and $\mathfrak{h}^{*}$

$$
\alpha \cdot \beta=(\alpha, \beta), \text { or } \alpha \cdot \beta=\langle\alpha, \beta\rangle \text {. }
$$

We define the norm square

$$
\|\alpha\|^{2}=\alpha \cdot \alpha .
$$

For a coroot $\beta^{\vee} \in Q^{\vee}$, we also define

$$
\left|\beta^{\vee}\right|=\sum_{i=1}^{\mathrm{rk}} \beta_{i}^{\vee}, \quad \text { with } \beta^{\vee}=\sum_{i=1}^{\mathrm{rk}} \beta_{i}^{\vee} \alpha_{i}^{\vee} .
$$

Besides, for a vector $m$ representing the Kähler paramters associated to a Lie algebra, we denote $m_{\alpha}=m \cdot \alpha$ for short.

Following the de-affinisation procedure described in section 2.4, we derive the elliptic blowup equations as

$$
\begin{aligned}
& \frac{1}{2}\|\omega\|^{2}+d_{1}+d_{2}=d \\
& \sum_{\omega \in \phi_{\lambda}\left(Q^{\vee}\right), d_{1,2} \in \mathbb{N}}(-1)^{\left|\phi_{\lambda}^{-1}(\omega)\right|} \cdot \theta_{i}^{[a]}\left(\mathfrak{n} \tau,(\mathfrak{n}-2)\left(\epsilon_{1}+\epsilon_{2}\right)-\mathfrak{n}\left(\left(\frac{1}{2}|| \omega \|^{2}+d_{1}\right) \epsilon_{1}+\left(\frac{1}{2}|| \omega \|^{2}+d_{2}\right) \epsilon_{2}-m_{\omega}\right)\right) \\
& \times A_{\omega}(m) \cdot \mathbb{E}_{d_{1}}\left(\tau, m-\epsilon_{1} \omega, \epsilon_{1}, \epsilon_{2}-\epsilon_{1}\right) \cdot \mathbb{E}_{d_{2}}\left(\tau, m-\epsilon_{2} \omega, \epsilon_{1}-\epsilon_{2}, \epsilon_{2}\right) \\
& = \begin{cases}\theta_{i}^{[a]}\left(\mathfrak{n} \tau,(\mathfrak{n}-2)\left(\epsilon_{1}+\epsilon_{2}\right)\right) \cdot \mathbb{E}_{d}\left(\tau, m, \epsilon_{1}, \epsilon_{2}\right), & \text { fixed } d \in \mathbb{N}, \\
0, & \text { fixed } d \notin \mathbb{N} .\end{cases}
\end{aligned}
$$

Here the subscript of theta functions $i$ is 4 if $\mathfrak{n}$ is odd and 3 if $\mathfrak{n}$ is even, and the characteristic $a=k / \mathfrak{n}-1 / 2, k=0,1, \ldots, \mathfrak{n}-1$. The factor $A_{\omega}(m)$ is given by

$$
A_{\omega}(m)=\prod_{\alpha \in \Delta_{+}} \breve{\theta}\left(m_{\alpha},-\alpha \cdot \omega\right)
$$

where we denote for $R \geq 0$,

$$
\breve{\theta}(z, R)=\prod_{\substack{m, n \geq 0 \\ m+n \leq R-1}} \frac{\eta}{\theta_{1}\left(z+m \epsilon_{1}+n \epsilon_{2}\right)} \prod_{\substack{m, n \geq 0 \\ m+n \leq R-2}} \frac{\eta}{\theta_{1}\left(z+(m+1) \epsilon_{1}+(n+1) \epsilon_{2}\right)},
$$


and for $R \leq 0, \breve{\theta}(z, R)=\left.\breve{\theta}(z,-R)\right|_{\epsilon_{1,2} \rightarrow-\epsilon_{1,2}}$. Note the $\alpha \cdot \omega$ in (3.5) is guaranteed to be an integer, as requested for the definition of $\breve{\theta}(z, R)$. See more about the origin of $\breve{\theta}(z, R)$ function in appendix D. Let us show some examples here:

$$
\begin{aligned}
\breve{\theta}(z, 0) & =1, \\
\breve{\theta}(z, \pm 1) & =\frac{\eta}{\theta_{1}(z)}, \\
\breve{\theta}(z, \pm 2) & =\frac{\eta^{4}}{\theta_{1}(z) \theta_{1}\left(z \pm \epsilon_{1}\right) \theta_{1}\left(z \pm \epsilon_{2}\right) \theta_{1}\left(z \pm \epsilon_{1} \pm \epsilon_{2}\right)} .
\end{aligned}
$$

The dependence on the $r$-field in (3.4) is related to the choice of $a$. If we choose basis of Kähler moduli $\left(t_{\mathrm{ell}}, \tau, m_{i}\right), i=1, \ldots, \mathrm{rk}$ as in $(2.4)$, the corresponding components of the $r$-field are $\left(r_{\text {ell }}, r_{\tau}, r_{i}\right), i=1, \ldots$, rk. The first component $r_{\text {ell }}$ controls the characteristic $a$ through $a=r_{\text {ell }} / 2 \mathfrak{n}$, thus the latter can take any of the following values

$$
a=-\frac{\mathfrak{n}-2 k}{2 \mathfrak{n}}, \quad k=0,1, \ldots, \mathfrak{n}-1 .
$$

The component $r_{\tau}$ vanishes due to the admissibility condition (2.22). The remaining components $r_{i}, i=1, \ldots$,rk always correspond to a weight vector $\lambda \in P$ through (2.28), which in turn induces the embedding $\phi_{\lambda}: Q^{\vee} \hookrightarrow P$, and the summation index vector $\omega \in \phi_{\lambda}\left(Q^{\vee}\right)$ plays the role of the shift vector $-R$. The number of different embeddings is $\left|P: Q^{\vee}\right|$. The total number of different blowup equations is then $\mathfrak{n}\left|P: Q^{\vee}\right|$, which explains the numerology found in (2.34).

\subsection{Modularity of elliptic blowup equations}

In this section, we provide evidence for the elliptic blowup equations (3.4) by showing that the components of the elliptic blowup equations transforms correctly as weak Jacobi forms. This is established by showing that the weight and the index, in general a quadratic polynomial, of the corresponding components in (3.4) match the predictions for the index and weight made from the $2 \mathrm{~d}$ and the $6 \mathrm{~d}$ anomaly polynomial or from the transformation properties of the refined topological string partition function under the $S$ and $T$ monodromies of the Calabi-Yau space $X$, see [22] and more generally [58]. In general the blowup equations give interesting identities for Jacobi forms, one example is proven in section 3.3.1, see also (3.48). In the fortuitous cases where the expressions of $\mathbb{E}_{d}$ are already known, for instance the $G=\mathrm{SU}(3), \mathrm{SO}(8)$ models $[8,34]$, we can plug in their expressions, and verify these identities by small $Q_{\tau}$ expansion.

It is easy to see that each term in the summation of (3.4) has weight $1 / 2$ as both $A_{\omega}$ and $\mathbb{E}_{d}$ are of weight zero and $\theta_{i}$ has weight $1 / 2$. The identification of the modular indices requires a bit of computation, which is independent from the characteristic $a$. The basic idea is to repeatedly use the fact that $\theta(N \tau, N z)$ is of index $N / 2$. Let us denote

$$
d_{0}=\frac{1}{2}\|\omega\|^{2} .
$$

It is easy to see the theta function $\theta_{i}^{[a]}$ on the left hand side has modular index

$$
\operatorname{Ind}_{d_{0}}^{G}=\frac{\mathfrak{n}}{2}\left(\left(d_{0}+d_{1}-\frac{\mathfrak{n}-2}{\mathfrak{n}}\right) \epsilon_{1}+\left(d_{0}+d_{2}-\frac{\mathfrak{n}-2}{\mathfrak{n}}\right) \epsilon_{2}-m_{\omega}\right)^{2} .
$$


Using (D.16), the modular index polynomial of $A_{\omega}(m)$ can be computed as

$$
\begin{aligned}
\operatorname{Ind}_{A}^{G}= & -\frac{\mathfrak{n}}{2} m_{\omega}^{2}-\frac{\mathfrak{n}}{2} d_{0} m \cdot m+\left(\mathfrak{n} d_{0}+2-\mathfrak{n}\right)\left(\epsilon_{1}+\epsilon_{2}\right) m_{\omega} \\
& -\frac{d_{0}}{2}\left(\mathfrak{n} d_{0}+2-\mathfrak{n}\right)\left(\epsilon_{1}^{2}+\epsilon_{1} \epsilon_{2}+\epsilon_{2}^{2}\right) .
\end{aligned}
$$

The elliptic genus $\mathbb{E}_{d}\left(\tau, m, \epsilon_{1}, \epsilon_{2}\right)$ is known to have the modular index [24,37]

$$
\operatorname{Ind}_{d}^{G}=-\frac{1}{2} d(\mathfrak{n}-2)\left(\epsilon_{1}+\epsilon_{2}\right)^{2}+\frac{1}{2} d(\mathfrak{n} d-\mathfrak{n}+2) \epsilon_{1} \epsilon_{2}-\frac{1}{2} \mathfrak{n} d m \cdot m .
$$

Thus the modular index polynomials of $\mathbb{E}_{d_{1}}\left(\tau, m-\epsilon_{1} \omega, \epsilon_{1}, \epsilon_{2}-\epsilon_{1}\right)$ and $\mathbb{E}_{d_{2}}\left(\tau, m-\epsilon_{2} \omega, \epsilon_{1}-\right.$ $\left.\epsilon_{2}, \epsilon_{2}\right)$ can be computed respectively as

$$
\begin{aligned}
\operatorname{Ind}_{d_{1}}^{G} & =\frac{1}{2}\left(-(\mathfrak{n}-2) d_{1} \epsilon_{2}^{2}+\left(\mathfrak{n} d_{1}-\mathfrak{n}+2\right) d_{1} \epsilon_{1}\left(\epsilon_{2}-\epsilon_{1}\right)-\mathfrak{n} d_{1}\left(m-\epsilon_{1} \omega\right)^{2}\right) \\
\operatorname{Ind}_{d_{2}}^{G} & =\frac{1}{2}\left(-(\mathfrak{n}-2) d_{2} \epsilon_{1}^{2}+\left(\mathfrak{n} d_{2}-(\mathfrak{n}-2)\right) d_{2}\left(\epsilon_{1}-\epsilon_{2}\right) \epsilon_{2}-\mathfrak{n} d_{2}\left(m-\epsilon_{2} \omega\right)^{2}\right)
\end{aligned}
$$

Using $d=\|\omega\|^{2} / 2+d_{1}+d_{2}$, we find that the four components on the left hand side of (3.4) has total modular index polynomial as

$$
\operatorname{Ind}_{d_{0}}^{G}+\operatorname{Ind}_{A}^{G}+\operatorname{Ind}_{d_{1}}^{G}+\operatorname{Ind}_{d_{2}}^{G}=-\frac{(\mathfrak{n}-2)(\mathfrak{n}-2+d \mathfrak{n})}{2 \mathfrak{n}}\left(\epsilon_{1}+\epsilon_{2}\right)^{2}+\frac{d(d \mathfrak{n}-\mathfrak{n}+2)}{2} \epsilon_{1} \epsilon_{2}-\frac{d \mathfrak{n}}{2} m \cdot m
$$

which is independent from $\omega, d_{1}, d_{2}$ individually but only depends on their combination $d$ ! This highly nontrivial fact guarantees the modularity of elliptic blowup equation, which means in summation of the left hand side of (3.4) all terms share the same modular index, thus transform as whole Jacobi form together! In the case that $d \notin \mathbb{N}$ where the right hand side of (3.4) vanishes, this is the end of the story. If $d \in \mathbb{N}$ so that the right hand side of (3.4) is non-vanishing, we still need to show the right hand side also shares the same index polynomial. The index polynomial of $\Lambda$ is simply,

$$
\operatorname{Ind}_{\Lambda}^{G}=\frac{(\mathfrak{n}-2)^{2}}{2 \mathfrak{n}}\left(\epsilon_{1}+\epsilon_{2}\right)^{2}
$$

which together with (3.12) indeed sum up to (3.15).

\subsection{Universality of elliptic blowup equations}

We demonstrate here an interesting property of the elliptic blowup equations. The $\mathfrak{n}$ blowup equations (3.4) with a fixed embedding $\phi_{\lambda}$ can be ordered by the characteristics $a$ of the theta functions, where for two consecutive equations $a$ differ by $1 / \mathfrak{n}$. We claim that if two consecutive unity blowup equations are valid, the other equations must hold automatically. We call this the universality of the elliptic blowup equations. ${ }^{17}$

\footnotetext{
${ }^{17}$ The following argument assumes the form of refined BPS expansion. Thus the universality here does not contradict with our statement that choosing arbitrary three unity $r$ fields, i.e. three different characteristics $a$, one is able to use the blowup equations to solve out the elliptic genus.
} 
Theta functions with characteristics have the following properties

$$
\begin{aligned}
& \theta_{3}^{\left[\frac{m}{2 \mathfrak{n}}\right]}(\mathfrak{n} \tau, z)=\mathrm{e}^{\pi \mathrm{i} \tau \frac{m^{2}}{4 \mathfrak{n}}+\pi \mathrm{i} z \frac{m}{\mathfrak{n}}} \theta_{3}\left(\mathfrak{n} \tau, z+\frac{1}{2} m \tau\right), \quad m \in \mathbb{Z}, \\
& \theta_{4}^{\left[\frac{m}{2 \mathfrak{n}}\right]}(\mathfrak{n} \tau, z)=\mathrm{e}^{\pi \mathrm{i} \frac{m}{2 \mathfrak{n}}+\pi \mathrm{i} \tau \frac{m^{2}}{4 \mathfrak{n}}+\pi \mathrm{i} z \frac{m}{\mathfrak{n}}} \theta_{4}\left(\mathfrak{n} \tau, z+\frac{1}{2} m \tau\right), \quad m \in \mathbb{Z} .
\end{aligned}
$$

Therefore, shifting $z$ by $\tau$ is equivalent to shifting the characteristic of these theta function by $1 / \mathfrak{n}$. Then starting from one unity blowup equation, let us shift $\epsilon_{1}$ by $\tau$ and check how various Jacobi forms in (3.4) change.

- $\theta_{i}^{[a]}(\mathfrak{n} \tau, \ldots)$ : the elliptic parameter changes by $-2 \tau+$ integer $\cdot \mathfrak{n} \tau$. The shift integer $\cdot \mathfrak{n} \tau$ can be removed at the expense of an additional exponential factor due to quasiperiodicity of the theta function, while the shift $-2 \tau$, as we have argued, is equivalent to shifting $a$ by $-2 / \mathfrak{n}$.

- $A_{\omega}(m)$ : it is a product of factors like $\theta_{1}\left(\tau, m_{\alpha}+m \epsilon_{1}+n \epsilon_{2}\right), m, n \in \mathbb{Z}$, therefore is invariant under this shift up to an exponential factor.

- $\mathbb{E}_{k}$ : we first argue that under the shift $\epsilon_{1} \rightarrow \epsilon_{1}+1, \mathbb{E}_{k}\left(\tau, m, \epsilon_{1}, \epsilon_{2}\right)$ is invariant up to an exponential factor. The refined BPS are defined from the topological string free energy as ${ }^{18}$

$$
F^{\text {inst }}\left(Q, \epsilon_{1}, \epsilon_{2}\right)=\sum_{j_{l}, j_{r} \geq 0} \sum_{w \geq 1, \beta}(-1)^{2\left(j_{l}+j_{r}\right)} N_{j_{l}, j_{r}}^{\beta} \frac{\chi_{j_{l}}\left(q_{l}^{w}\right) \chi_{j_{r}}\left(q_{r}^{w}\right)}{w\left(q_{1}^{w / 2}-q_{1}^{-w / 2}\right)\left(q_{2}^{w / 2}-q_{2}^{-w / 2}\right)} Q^{w \beta}
$$

where

$$
\chi_{j}(q)=\frac{q^{2 j+1}-q^{-2 j-1}}{q-q^{-1}} .
$$

Exponentiating (3.19) the instanton partition function reads

$$
Z^{\mathrm{inst}}\left(Q, \epsilon_{1}, \epsilon_{2}\right)=\prod_{\beta} \prod_{j_{l / r}=0}^{\infty} \prod_{m_{l / r}=-j_{l / r}}^{j_{l / r}} \prod_{m_{1}, m_{2}=1}^{\infty}\left(1-q_{l}^{m_{l}} q_{r}^{m_{r}} q_{1}^{m_{1}-\frac{1}{2}} q_{2}^{m_{2}-\frac{1}{2}} Q^{\beta}\right)^{(-1)^{2\left(j_{l}+j_{r}\right)} N_{j_{l} j_{r}}^{\beta}} .
$$

Using (3.19) and the checkerboard pattern identity

$$
2 j_{l}+2 j_{r}+1 \equiv r \cdot \beta \quad(\bmod 2)
$$

for non-vanishing BPS invariants one can show that the refined BPS partition function is invariant under the combined transformation

$$
\left(\epsilon_{1}, \epsilon_{2}, t\right) \rightarrow\left(\epsilon_{1}+1, \epsilon_{2}, t+r\right)
$$

Since the $r$-vector for the minimal $6 \mathrm{~d}$ SCFTs in terms of the Kähler moduli $t_{I}, t_{B}$ have components

$$
r \equiv(0, \ldots, 0, \mathfrak{n}) \quad \bmod (2 \mathbb{Z})^{\mathrm{rk}+2},
$$

\footnotetext{
${ }^{18}$ Here $q_{j} \equiv \exp \left(2 \pi i \epsilon_{j}\right) j=1,2$ and $q_{l} \equiv \sqrt{q_{1} / q_{2}}, q_{r} \equiv \sqrt{q_{1} q_{2}}$. We will also use $v \equiv q_{r}, x \equiv q_{l}$ in section 4 and 5 to make contact with the literature such as [37, 38].
} 
the elliptic genus $\mathbb{E}_{k}\left(\tau, m, \epsilon_{1}, \epsilon_{2}\right)$ is invariant under the shift $\epsilon_{1} \rightarrow \epsilon_{1}+1$, at most up to a sign if $\mathfrak{n}$ is odd. In fact

$$
\mathbb{E}_{k}\left(\tau, m, \epsilon_{1}+1, \epsilon_{2}\right)=(-1)^{\mathfrak{n} k} \mathbb{E}\left(\tau, m, \epsilon_{1}, \epsilon_{2}\right) .
$$

Together with the modular property of $\mathbb{E}_{k}$, this implies $\mathbb{E}_{k}\left(\tau, m, \epsilon_{1}, \epsilon_{2}\right)$ is quasiperiodic for $\epsilon_{1} \rightarrow \epsilon_{1}+\tau$. Similarly, one can show that $\mathbb{E}_{k}\left(\tau, m, \epsilon_{1}, \epsilon_{2}\right)$ is quasi-periodic for $\epsilon_{2} \rightarrow \epsilon_{2}+\tau$ as well. As examples one could inspect the expressions of $\mathbb{E}_{k}$ for the $\mathfrak{n}=3,4$ models $[8,34]$, which are composed of $\theta_{1}\left(\tau\right.$, integer $\cdot \epsilon_{1}+$ integer $\left.\cdot \epsilon_{2}+\ldots\right)$ and therefore are indeed quasi-periodic for $\epsilon_{1,2} \rightarrow \epsilon_{1,2}+\tau$. Now if we forget for the moment the shift on the mass parameters, the three instances of elliptic genera $\mathbb{E}_{k}\left(\tau, m-\epsilon_{1} \omega, \epsilon_{1}, \epsilon_{2}-\epsilon_{1}\right), \mathbb{E}_{k}\left(\tau, m-\epsilon_{2} \omega, \epsilon_{1}-\epsilon_{2}, \epsilon_{2}\right), \mathbb{E}_{k}\left(\tau, m, \epsilon_{1}, \epsilon_{2}\right)$ in (3.4) should already be invariant under $\epsilon_{1} \rightarrow \epsilon_{1}+\tau$ up to an exponential factor. The shift on the mass parameters $m-\epsilon_{1} \omega$ in the first instance of $\mathbb{E}_{k}$ means that upon $\epsilon_{1} \rightarrow \epsilon_{1}+\tau$ its elliptic parameter is in addition shifted by $\tau$ times a weight vector, which can also be removed at the expense of an additional exponential factor [24]. ${ }^{19}$

In summary, the shift $\epsilon_{1} \rightarrow \epsilon_{1}+\tau$ is equivalent to shifting the characteristic $a$ of the theta functions $\theta_{i}^{[a]}(\mathfrak{n} \tau, \ldots)$ by $-2 / \mathfrak{n}$ and in addition multiplying each term in (3.4) by an exponential factor. These exponential factors are determined by the index polynomial of each term, which as a consequence of section 3.1, should be identical. Thus all the exponential factors can be factored out and removed, and we are left again with a unity blowup equation where the characteristic is shifted by $-2 / \mathfrak{n}$. This immediately indicates that starting from two consecutive unity blowup equations, we can obtain all the other unity blowup equations, hence the universality property.

\subsection{Unity blowup equations}

The elliptic blowup equations depend on the choice of the weight vector $\lambda$ and they take different forms depending on if $\lambda \in Q^{\vee}$ or not. We first consider the former case where $\phi_{\lambda}\left(Q^{\vee}\right)$ coincides with $Q^{\vee}$. We can denote $\omega$ as vector $\beta^{\vee}$ in the coroot lattice. Then $\left\|\beta^{\vee}\right\|^{2} / 2$ and thus $d$ are always nonnegative integers, and as a result the right hand side of (3.4) does not vanish

$$
\begin{aligned}
\sum_{\beta^{\vee} \in Q^{\vee}, d_{1,2} \in \mathbb{N}}^{\frac{1}{2}\left\|\beta^{\vee}\right\|^{2}+d_{1}+d_{2}=d} & (-1)^{\left|\beta^{\vee}\right|} \theta_{i}^{[a]}\left(\mathfrak{n} \tau,(\mathfrak{n}-2)\left(\epsilon_{1}+\epsilon_{2}\right)-\mathfrak{n}\left(\left(\frac{1}{2}\left\|\beta^{\vee}\right\|^{2}+d_{1}\right) \epsilon_{1}+\left(\frac{1}{2}\left\|\beta^{\vee}\right\|^{2}+d_{2}\right) \epsilon_{2}-m_{\beta^{\vee}}\right)\right) \\
& \times A_{\beta^{\vee}}(m) \mathbb{E}_{d_{1}}\left(\tau, m-\epsilon_{1} \beta^{\vee}, \epsilon_{1}, \epsilon_{2}-\epsilon_{1}\right) \mathbb{E}_{d_{2}}\left(\tau, m-\epsilon_{2} \beta^{\vee}, \epsilon_{1}-\epsilon_{2}, \epsilon_{2}\right) \\
= & \theta_{i}^{[a]}\left(\mathfrak{n} \tau,(\mathfrak{n}-2)\left(\epsilon_{1}+\epsilon_{2}\right)\right) \cdot \mathbb{E}_{d}\left(\tau, m, \epsilon_{1}, \epsilon_{2}\right), \quad d \in \mathbb{N} .
\end{aligned}
$$

We say these elliptic blowup equations are of the unity type following the nomenclature in [15]. Since the number of embedding $Q^{\vee} \hookrightarrow \phi_{\lambda}\left(Q^{\vee}\right)=Q^{\vee}$ is unique, the number of unity

\footnotetext{
${ }^{19}$ It is established in section 4 of [24] that the elliptic genera of $6 \mathrm{~d}$ SCFTs with a pure gauge bulk theory in fact consist of special Weyl invariant Jacobi forms, which, among other things, are quasi-periodic if the elliptic parameter is shifted by $\tau$ times a weight vector.
} 
blowup equations is the same as the range of characteristics $a$, which is $\mathfrak{n}$. We also point out that, using the property that the leading $Q_{\tau}$ order of $\mathbb{E}_{d}$ is $-d h_{G}^{\vee} / 6,{ }^{20}$ these equations in the leading order of $Q_{\tau}$ boil down to the identity

$$
\sum_{\alpha \in \Delta^{+}(G)}(\beta \cdot \alpha)^{2}=h_{G}^{\vee}\|\beta\|^{2},
$$

which is guaranteed by the Lie algebraic identity (A.5).

The unity blowup equations are particular interesting as they allow us to write down recursion formulas for the elliptic genera.

\subsubsection{Recursion formulas for elliptic genera}

The unity blowup equations (3.26) can be put in the following more suggestive form

$$
\begin{aligned}
& \theta_{i}^{[a]}\left(\mathfrak{n} \tau,(\mathfrak{n}-2-d \mathfrak{n}) \epsilon_{1}+(\mathfrak{n}-2) \epsilon_{2}\right) \mathbb{E}_{d}\left(\tau, m, \epsilon_{1}, \epsilon_{2}-\epsilon_{1}\right) \\
& \quad+\theta_{i}^{[a]}\left(\mathfrak{n} \tau,(\mathfrak{n}-2) \epsilon_{1}+(\mathfrak{n}-2-d \mathfrak{n}) \epsilon_{2}\right) \mathbb{E}_{d}\left(\tau, m, \epsilon_{1}-\epsilon_{2}, \epsilon_{2}\right) \\
& \quad-\theta_{i}^{[a]}\left(\mathfrak{n} \tau,(\mathfrak{n}-2)\left(\epsilon_{1}+\epsilon_{2}\right)\right) \mathbb{E}_{d}\left(\tau, m, \epsilon_{1}, \epsilon_{2}\right)=I_{d}\left(\mathbb{E}_{<d}\right) .
\end{aligned}
$$

where $I_{d}\left(\mathbb{E}_{<d}\right)$ only contains the elliptic genera of degrees lower than $d$. Since three copies of $\mathbb{E}_{d}$ on the left hand side do not depend on the characteristic $a$, if we have three such equations with different $a$, which is indeed the case for all the minimal $6 \mathrm{~d} \mathcal{N}=(1,0)$ SCFTs with pure gauge bulk theory, we can solve $\mathbb{E}_{d}\left(\tau,, \epsilon_{1}, \epsilon_{2}\right)$ in terms of elliptic genera with lower number of strings; in other words, we obtain recursion formulas for elliptic genera.

Let us use the short hand notation ${ }^{21}$

$$
\theta_{i,\left\{d_{0}, d_{1}, d_{2}\right\}}^{[a]}=\theta_{i}^{[a]}\left(\mathfrak{n} \tau, \mathfrak{n} m_{\alpha} \vee+(\mathfrak{n}-2)\left(\epsilon_{1}+\epsilon_{2}\right)-\mathfrak{n}\left(\left(d_{0}+d_{1}\right) \epsilon_{1}+\left(d_{0}+d_{2}\right) \epsilon_{2}\right)\right),
$$

where $m_{\alpha^{\vee}}=m \cdot \alpha^{\vee}$, and furthermore define

$$
D_{d}=\operatorname{Det}\left(\begin{array}{ccc}
\theta_{i,\{0, d, 0\}}^{\left[a_{1}\right]} & \theta_{i,\{0,0, d\}}^{\left[a_{1}\right]} & \theta_{i,\{0,0,0\}}^{\left[a_{1}\right]} \\
\theta_{i,\{0, d, 0\}}^{\left[a_{2}\right]} & \theta_{i,\{0,0, d\}}^{\left[a_{2}\right]} & \theta_{i,\{0,0,0\}}^{\left[a_{2}\right]} \\
\theta_{i,\{0, d, 0\}}^{\left[a_{3}\right]} & \theta_{i,\{0,0, d\}}^{\left[a_{3}\right]} & \theta_{i,\{0,0,0\}}^{\left[a_{3}\right]}
\end{array}\right)
$$

as well as

$$
D_{\left\{d_{0}, d_{1}, d_{2}\right\}}^{\alpha^{\vee}}=\operatorname{Det}\left(\begin{array}{ccc}
\theta_{i,\{0, d, 0\}}^{\left[a_{1}\right]} & \theta_{i,\{0,0, d\}}^{\left[a_{1}\right]} & \theta_{i,\left\{d_{0}, d_{1}, d_{2}\right\}}^{\left[a_{1}\right]} \\
\theta_{i,\{0, d, 0\}}^{\left[a_{2}\right]} & \theta_{i,\{0,0, d\}}^{\left[a_{2}\right]} & \theta_{i,\left\{d_{0}, d_{1}, d_{2}\right\}}^{\left[a_{2}\right]} \\
\theta_{i,\{0, d, 0\}}^{\left[a_{3}\right]} & \theta_{i,\{0,0, d\}}^{\left[a_{3}\right]} & \theta_{i,\left\{d_{0}, d_{1}, d_{2}\right\}}^{\left[a_{3}\right]}
\end{array}\right) .
$$

Note that $D_{d}=D_{\{0,0,0\}}^{\alpha^{\vee}}$ does not depend on $\alpha^{\vee}$ since $\alpha^{\vee}=0$ when $d_{0}=\left\|\alpha^{\vee}\right\|^{2} / 2=0$. Then the recursion formulas of $\mathbb{E}_{d}$ solved from (3.28) read

$$
\mathbb{E}_{d}=\sum_{d_{0}=\frac{1}{2}\left\|\alpha^{\vee}\right\|^{2}, d_{1,2}<d}^{d_{0}+d_{1}+d_{2}=d}(-1)^{\left|\alpha^{\vee}\right|} \frac{D_{\left\{d_{0}, d_{1}, d_{2}\right\}}^{\alpha^{\vee}}}{D_{d}} A_{\alpha \vee}(m) \mathbb{E}_{d_{1}}\left(m-\epsilon_{1} \alpha^{\vee}, \epsilon_{1}, \epsilon_{2}-\epsilon_{1}\right) \mathbb{E}_{d_{2}}\left(m-\epsilon_{2} \alpha^{\vee}, \epsilon_{1}-\epsilon_{2}, \epsilon_{2}\right) .
$$

Here the $\tau$ dependence is implied.

\footnotetext{
${ }^{20}$ See more discussion in section 4.1 .

${ }^{21}$ Note $d_{0}=\left\|\alpha^{\vee}\right\|^{2} / 2$ is always implied. When $d_{0}=0, \theta_{i,\left\{d_{0}, d_{1}, d_{2}\right\}}^{[a]}$ does not depend on $\alpha^{\vee}$ since $\alpha^{\vee}=0$.
} 
Let us look at some examples. The one-string elliptic genus is given by

$$
\mathbb{E}_{1}=\sum_{\substack{\alpha \in \Delta^{\vee} \\\left\|\alpha^{\vee}\right\|^{2}=2}} \frac{D_{\{1,0,0\}}^{\alpha^{\vee}}}{D_{1}} \frac{\eta^{4}}{\theta_{1}\left(m_{\alpha}\right) \theta_{1}\left(m_{\alpha}-\epsilon_{1}\right) \theta_{1}\left(m_{\alpha}-\epsilon_{2}\right) \theta_{1}\left(m_{\alpha}-\epsilon_{1}-\epsilon_{2}\right)} \prod_{\substack{\beta \in \Delta \\ \alpha^{\vee} \cdot \beta=1}} \frac{\eta}{\theta_{1}\left(m_{\beta}\right)}
$$

where $m_{\beta}=m \cdot \beta$. In particular, for ADE type algebras, the $\mathbb{E}_{1}$ formula can be further simplified due to the identification of roots and coroots. Indeed, for $A_{2}, D_{4}, E_{6,7,8}$, we have the following universal formula

$$
\mathbb{E}_{1}=\sum_{\alpha \in \Delta} \frac{D_{\alpha}}{D} \frac{\eta^{4}}{\theta_{1}\left(m_{\alpha}\right) \theta_{1}\left(m_{\alpha}-\epsilon_{1}\right) \theta_{1}\left(m_{\alpha}-\epsilon_{2}\right) \theta_{1}\left(m_{\alpha}-\epsilon_{1}-\epsilon_{2}\right)} \prod_{\substack{\beta \in \Delta \\ \alpha \cdot \beta=1}} \frac{\eta}{\theta_{1}\left(m_{\beta}\right)} .
$$

Here $D_{\alpha}$ and $D$ are the short notations for $D_{\{1,0,0\}}^{\alpha}$ and $D_{1}$.

In the $Q_{\tau} \rightarrow 0$ limit, $\mathbb{E}_{1}$ formula (3.33) reduces to the universal one-instanton partition function of $5 \mathrm{~d} \mathcal{N}=1$ pure SYM theory [30, 59]

$$
Z_{1}=\frac{1}{\left(1-\mathrm{e}^{-\epsilon_{1}}\right)\left(1-\mathrm{e}^{-\epsilon_{2}}\right)} \sum_{\gamma \in \Delta_{l}} \frac{\mathrm{e}^{\left(h_{G}^{\vee}-1\right) m_{\gamma} / 2}}{\left(1-\mathrm{e}^{-\epsilon_{1}-\epsilon_{2}+m_{\gamma}}\right)\left(\mathrm{e}^{m_{\gamma} / 2}-\mathrm{e}^{-m_{\gamma} / 2}\right) \prod_{\alpha \cdot \gamma=1}\left(\mathrm{e}^{m_{\alpha} / 2}-\mathrm{e}^{-m_{\alpha} / 2}\right)},
$$

where $\Delta_{l}$ denotes the set of long roots which is the same with coroots with $\left\|\alpha^{\vee}\right\|^{2}=2$.

Furthermore, the two-string elliptic genus is given by

$$
\begin{aligned}
& \mathbb{E}_{2}=\frac{D_{\{0,1,1\}}^{\alpha^{\vee}=0}}{D_{2}} \mathbb{E}_{1}\left(m, \epsilon_{1}, \epsilon_{2}-\epsilon_{1}\right) \mathbb{E}_{1}\left(m, \epsilon_{1}-\epsilon_{2}, \epsilon_{2}\right)+\sum_{\left\|\alpha^{\vee}\right\|^{2}=4}(-1)^{\left|\alpha^{\vee}\right|} A_{\alpha^{\vee}}(m) \frac{D_{\{2,0,0\}}^{\alpha^{\vee}}}{D_{2}} \\
& +\sum_{\left\|\alpha^{\vee}\right\|^{2}=2}(-1)^{\left|\alpha^{\vee}\right|} A_{\alpha \vee}(m)\left(\frac{D_{\{1,1,0\}}^{\alpha^{\vee}}}{D_{2}} \mathbb{E}_{1}\left(m-\epsilon_{1} \alpha^{\vee}, \epsilon_{1}, \epsilon_{2}-\epsilon_{1}\right)+\frac{D_{\{1,0,1\}}^{\alpha^{\vee}}}{D_{2}} \mathbb{E}_{1}\left(m-\epsilon_{2} \alpha^{\vee}, \epsilon_{1}-\epsilon_{2}, \epsilon_{2}\right)\right) .
\end{aligned}
$$

Note in the bracket of the second line of (3.36), the two terms are symmetric in $\epsilon_{1} \leftrightarrow \epsilon_{2}$. In the later section, we use this formula to compute the two-string elliptic genus of all $6 \mathrm{~d}$ $(1,0)$ minimal SCFTs with $G=A_{2}, D_{4}, F_{4}, E_{6,7,8}$. From (3.32), we can also easily write down the universal formula for three-string elliptic genus as

$$
\begin{aligned}
\mathbb{E}_{3}= & {\left[\frac{D_{\{0,1,2\}}^{\alpha^{\vee}=0}}{D_{3}} \mathbb{E}_{1}\left(m, \epsilon_{1}, \epsilon_{2}-\epsilon_{1}\right) \mathbb{E}_{2}\left(m, \epsilon_{1}-\epsilon_{2}, \epsilon_{2}\right)+\left(\epsilon_{1} \leftrightarrow \epsilon_{2}\right)\right]+\sum_{\left\|\alpha^{\vee}\right\|^{2}=6}(-1)^{\left|\alpha^{\vee}\right|} A_{\alpha \vee}(m) \frac{D_{\{3,0,0\}}^{\alpha^{\vee}}}{D_{3}} } \\
& +\sum_{\left\|\alpha^{\vee}\right\|^{2}=4}(-1)^{\left|\alpha^{\vee}\right|} A_{\alpha \vee}(m)\left[\frac{D_{\{2,1,0\}}^{\alpha^{\vee}}}{D_{3}} \mathbb{E}_{1}\left(m-\epsilon_{1} \alpha^{\vee}, \epsilon_{1}, \epsilon_{2}-\epsilon_{1}\right)+\left(\epsilon_{1} \leftrightarrow \epsilon_{2}\right)\right] \\
& +\sum_{\left\|\alpha^{\vee}\right\|^{2}=2}(-1)^{\left|\alpha^{\vee}\right|} A_{\alpha \vee}(m)\left[\left(\frac{D_{\{1,2,0\}}^{\alpha^{\vee}}}{D_{3}} \mathbb{E}_{2}\left(m-\epsilon_{1} \alpha^{\vee}, \epsilon_{1}, \epsilon_{2}-\epsilon_{1}\right)+\left(\epsilon_{1} \leftrightarrow \epsilon_{2}\right)\right)\right. \\
& \left.\quad \frac{D_{\{1,1,1\}}^{\alpha^{\vee}}}{D_{3}} \mathbb{E}_{1}\left(m-\epsilon_{1} \alpha^{\vee}, \epsilon_{1}, \epsilon_{2}-\epsilon_{1}\right) \mathbb{E}_{1}\left(m-\epsilon_{2} \alpha^{\vee}, \epsilon_{1}-\epsilon_{2}, \epsilon_{2}\right)\right] .
\end{aligned}
$$


From the topological string point of view, the $d$-string elliptic genus $\mathbb{E}_{d}$ encodes the BPS invariants $N_{j_{l}, j_{r}}^{\beta}$ with base degree $d$ as well as multi-wrapping contributions from lower base degree curves. Once all the elliptic genera up to certain base degree $d$ are computed, all the BPS invariants up to base degree $d$ and arbitrary degrees along other directions can be extracted. The recurison formulas (3.32) thus allow us to reproduce the genus zero Gopakumar-Vafa invariants for the Calabi-Yau threefolds associated to the minimal 6d SCFTs with $\mathfrak{n}=5,6,8,12$ [24], and to compute the refined BPS invariants for the first time in the literature.

Let us make a remark here concerning the validity of the recursion formula (3.32). Obviously, the recursion formula is only well-defined when $D_{d} \neq 0$. We have checked that this is indeed true for all the minimal models except for the model of $G=\mathrm{SU}(3)$ with $d=1$, where both $D_{\{1,0,0\}}^{\alpha}$ and $D_{1}$ vanish. This is a special situation since for the model with $G=\mathrm{SU}(3)$ there are only three choices of the characteristics $a_{i}$ and there may be certain symmetry enhancement for $\mathbb{E}_{1}$ such that only two of the three unity blowup equations are linearly independent. Note that this does not contradict with the fact that the universal one-instanton partition function $Z_{1}$ of $5 \mathrm{~d}$ pure SYM theories [30, 59] works perfectly for the $\mathrm{SU}(3)$ theory which is recovered from one-string elliptic genus in the 5 d limit with $Q_{\tau} \rightarrow 0$. What happens in this limit is that the $6 \mathrm{~d}$ unity $r$-field $r=(0,0,0,3)$ splits to two $5 \mathrm{~d}$ unity $r$-fields $r_{1}$ and $r_{2}$, and the leading $Q_{\tau}$ order term of $D_{1}$ is the difference of two contributions associated to $r_{1}$ and $r_{2}$ respectively, both of which remain finite and identical. Nevertheless, it should be emphasize that though recursion formula does not work for $\mathbb{E}_{1}^{\mathrm{SU}(3)}$, by assuming the refined BPS expansion, one can still use two unity blowup equations and one vanishing blowup equation to solve out all the refined BPS invariants, which is what we have done in [25]. If further assuming the knowledge on $Z_{0}$, one can actually use one single unity blowup equations to solve out all refined BPS invariants. To compute $\mathbb{E}_{2}^{\mathrm{SU}(3)}$ by recursion formula (3.36), practically one can use the exact formula $\mathbb{E}_{1}^{\mathrm{SU}(3)}$ in [34]. For all $d>1$, the recursion formula (3.32) works well for $\mathrm{SU}(3)$.

The identity $D_{1}^{\mathrm{SU}(3)}=0$ here despite its simple form does not seem so trivial. In fact, we find it is a special case of the following series of identities. With $q=\mathrm{e}^{\pi \mathrm{i} \tau}$ and $y=\mathrm{e}^{2 \pi \mathrm{i} z}$ we define the following double indexed $\theta$ functions

$$
\begin{aligned}
& \theta_{3}^{[r / 2 n]}(n \tau, z)=q^{\frac{r^{2}}{4 n}} y^{\frac{r}{2 n}} \sum_{l \in \mathbb{Z}} q^{l(n l+r)} y^{l}=\sum_{k=r(2 n)} q^{\frac{k^{2}}{4 n}} y^{\frac{k}{2 n}} \\
& \theta_{4}^{[r / 2 n]}(n \tau, z)=\mathrm{e}^{\frac{\pi i r}{4 n}} q^{\frac{r^{2}}{2 n}} y^{\frac{r}{2 n}} \sum_{l \in \mathbb{Z}}(-1)^{l} q^{l(n l+r)} y^{l}=\sum_{k=r(2 n)} q^{\frac{k^{2}}{4 n}}(-y)^{\frac{k}{2 n}}
\end{aligned}
$$

where the notations $k=r(m)$ in the second sums, mean that $k$ runs over values of the form $r+m l$, for any $l \in \mathbb{Z}$. We claim that if

$$
\sum_{j=0}^{n-1} z_{i}=0 \quad \leftrightarrow \quad \prod_{j=0}^{n-1} y_{j}=1
$$


the determinant of the $n \times n$ matrices defined from these $\theta$ functions

$$
\begin{aligned}
\operatorname{det}\left[\theta_{3}^{\left[\frac{i}{n}\right]}\left(n \tau, z_{j}\right)_{i, j=0, \ldots, n-1}\right] & =0, \quad \text { if } \quad 2 \mid n \\
\operatorname{det}\left[\theta_{4}^{\left[\frac{2 i+1}{2 n}\right]}\left(n \tau, z_{j}\right)_{i, j=0, \ldots, n-1}\right] & =0, \quad \text { if } \quad 2 \nmid n
\end{aligned}
$$

vanish. Note the $n=1$ case is just the well-known fact $\theta_{1}(\tau, 0)=0$, while $n=3$ case implies the $D_{1}^{\mathrm{SU}(3)}=0$ identity in our previous context. The proof proceeds in both cases in (3.40) by showing that each term of the form $q^{m} \prod_{j=1}^{n} y_{j}^{k_{j}}$ that occur in the expansion of the determinant, constrained by (3.39), appears once with positive and once with negative sign. ${ }^{22}$ The first case is notationally simpler so we prove it explicitly. Using $2 \mid n$ and an irrelevant rescaling ${ }^{23} z_{i} \rightarrow \tilde{z}_{i}=n z_{i}$ we rewrite the first determinate in the statement in $(3.40)$ as

$$
\begin{aligned}
\operatorname{det}\left[\left(\sum_{k=i(n)} q^{\frac{k^{2}}{n}} y_{j}^{k}\right)_{i, j=0, \ldots, n-1}\right] & =\sum_{\Pi} \operatorname{sgn}(\Pi) \sum_{\substack{k_{0}=\Pi(0)(n) \\
\vdots \\
k_{n-1} \stackrel{=}{=} \Pi(n-1)(n)}} q^{\frac{k_{0}^{2}+\ldots+k_{n-1}^{2}}{n}} y_{0}^{k_{0}} \ldots y_{n-1}^{k_{n-1}} \\
& =\sum_{\substack{k_{0}, \ldots, k_{n-1} \in \mathbb{Z} \\
k_{i} \neq k_{l} \bmod n}} \operatorname{sgn}\left(P_{k}\right) q^{\frac{k_{0}^{2}+\ldots+k_{n-1}^{2}}{n}} y_{0}^{k_{0}} \ldots y_{n-1}^{k_{n-1}} .
\end{aligned}
$$

Here $P_{k}$ is defined by the maps $i \mapsto k_{i} \bmod n$, for $i=0, \ldots, n-1$. Let $\bar{k}=\left(k_{0}+\ldots+k_{n-1}\right) / n$ be the average of $k_{i}$ and use it to define $k_{i}^{*}=k_{i}-2 \bar{k}$. Since $2 \mid n, \bar{k}$ runs over $\bar{k}=\frac{1}{2}(1)$ and hence $k_{i}^{*} \in \mathbb{Z}$. Direct calculation shows that under the $*$ operation $k_{i} \mapsto k_{i}^{*}$ the terms $m_{k}:=q^{\left(k_{0}^{2}+\ldots+k_{n-1}^{2}\right) / n} \prod_{j=0}^{n-1} y_{j}^{k_{j}}$ in (3.40) stay invariant $m_{k}=m_{k^{*}}$; the second factor due to (3.39). Let now $P_{k}^{*}$ be defined by $i \mapsto k_{i}^{*} \bmod n$. It follows immediately that it likewise defines a permutation of the indices $\{0, \ldots, n-1\}$, however with the opposite parity. Hence $m_{k}$ appears twice with opposite sign and the sum (3.41) is zero. The proof of the second case in (3.40) proceeds analogously, with appropriate relabelling of the indices.

\subsubsection{Uniqueness of recursion formulas}

One important consequence of the universality property is that the recursion formula (3.32) does not depend on the choice of three different $r$-fields in its construction, as it should. Let us supppose that we already known $\mathbb{E}_{<d}$ and we wish to compute $\mathbb{E}_{d}$ using the recursion formula (3.32) obtained from three consecutive unity blowup equations forming the linear system

$$
\left(\begin{array}{ccc}
\theta_{i,\{0, d, 0\}}^{\left[a_{1}\right]} & \theta_{i,\{0,0, d\}}^{\left[a_{1}\right]} & \theta_{i,\{0,0,0\}}^{\left[a_{1}\right]} \\
\theta_{i,\{0, d, 0\}}^{\left.a_{2}\right]} & \theta_{i,\{0,0, d\}}^{\left[a_{2}\right]} & \theta_{i,\{0,0,0\}}^{\left[a_{2}\right]} \\
\theta_{i,\{0, d, 0\}}^{\left.\left[a_{3}\right], 0\right\}} & \theta_{i,\{0,0, d\}}^{\left[a_{3}\right]} & \theta_{i,\{0,0,0\}}^{\left[a_{3}\right]}
\end{array}\right) \cdot\left(\begin{array}{c}
\mathbb{E}_{d}\left(m, \epsilon_{1}, \epsilon_{2}-\epsilon_{1}\right) \\
\mathbb{E}_{d}\left(m, \epsilon_{1}-\epsilon_{2}, \epsilon_{2}\right) \\
-\mathbb{E}_{d}\left(m, \epsilon_{1}, \epsilon_{2}\right)
\end{array}\right)=-\left(\begin{array}{c}
I_{d}^{\left[a_{1}\right]}\left(\mathbb{E}_{<d}\right) \\
I_{d}^{\left[a_{2}\right]}\left(\mathbb{E}_{<d}\right) \\
I_{d}^{\left[a_{3}\right]}\left(\mathbb{E}_{<d}\right)
\end{array}\right)
$$

\footnotetext{
${ }^{22}$ We thank Don Zagier for pointing this mechanism out to us.

${ }^{23}$ Followed by a renaming of the $\tilde{z}_{i}$ to $z_{i}$ again.
} 
where $a_{2}-a_{1}=a_{3}-a_{2}=1 / \mathfrak{n}$, and the matrix of theta function on the l.h.s. is of full rank. If the $\mathbb{E}_{d}\left(m, \epsilon_{1}, \epsilon_{2}\right)$ solved from the linear system is correct, so should be $\mathbb{E}_{d}\left(m, \epsilon_{1}, \epsilon_{2}-\epsilon_{1}\right)$, $\mathbb{E}_{d}\left(m, \epsilon_{1}-\epsilon_{2}, \epsilon_{2}\right)$, and the three unity blowup equations in (3.42) should all be correct as well. Otherwise, the linear system could always be corrected by

$$
\left(\begin{array}{ccc}
\theta_{i,\{0, d, 0\}}^{\left[a_{1}\right]} & \theta_{i,\{0,0, d\}}^{\left[a_{1}\right]} & \theta_{i,\{0,0,0\}}^{\left[a_{1}\right]} \\
\theta_{i,\{0, d, 0\}}^{\left[a_{2}\right]} & \theta_{i,\{0,0, d\}}^{\left[a_{2}\right]} & \theta_{i,\{0,0,0\}}^{\left[a_{2}\right]} \\
\theta_{i,\{0, d, 0\}}^{\left[a_{3}\right]} & \theta_{i,\{0,0, d\}}^{\left[a_{3}\right]} & \theta_{i,\{0,0,0\}}^{\left[a_{3}\right]}
\end{array}\right) \cdot\left(\begin{array}{c}
\mathbb{E}_{d}\left(m, \epsilon_{1}, \epsilon_{2}-\epsilon_{1}\right) \\
\mathbb{E}_{d}\left(m, \epsilon_{1}-\epsilon_{2}, \epsilon_{2}\right) \\
-\mathbb{E}_{d}\left(m, \epsilon_{1}, \epsilon_{2}\right)
\end{array}\right)=-\left(\begin{array}{c}
I_{d}^{\left[a_{1}\right]}\left(\mathbb{E}_{<d}\right) \\
I_{d}^{\left[a_{2}\right]}\left(\mathbb{E}_{<d}\right) \\
I_{d}^{\left[a_{3}\right]}\left(\mathbb{E}_{<d}\right)
\end{array}\right)+\left(\begin{array}{c}
R_{1} \\
R_{2} \\
R_{3}
\end{array}\right) .
$$

By inverting the matrix of theta functions in (3.42) and (3.43) and subtracting the two equations from each other, we get

$$
\left(\begin{array}{lll}
\theta_{i,\{0, d, 0\}}^{\left[a_{1}\right]} & \theta_{i,\{0,0, d\}}^{\left[a_{1}\right]} & \theta_{i,\{0,0,0\}}^{\left[a_{1}\right]} \\
\theta_{i,\{0, d, 0\}}^{\left[a_{2}\right]} & \theta_{i,\{0,0, d\}}^{\left[a_{2}\right]} & \theta_{i,\{0,0,0\}}^{\left[a_{2}\right]} \\
\theta_{i,\{0, d, 0\}}^{\left[a_{3}\right]} & \theta_{i,\{0,0, d\}}^{\left[a_{3}\right]} & \theta_{i,\{0,0,0\}}^{\left[a_{3}\right]}
\end{array}\right)^{-1} \cdot\left(\begin{array}{c}
R_{1} \\
R_{2} \\
R_{3}
\end{array}\right)=0
$$

which means the corrections $R_{1,2,3}$ must all vanish, as a consequence of $D_{d} \neq 0$. Once the validity of the three unity blowup equations in (3.42) is established, using the universality we can argue for the validity of all unity blowup equations. The recursion formula constructed out of any three unity blowup equations then should always gives the correct $\mathbb{E}_{d}$ which coincides with the solution of (3.42).

\subsection{Vanishing blowup equations}

We consider here the case where $\lambda \in P \backslash Q^{\vee}$. This is only possible if $\left|P: Q^{\vee}\right|>1$, i.e. for the minimal $6 \mathrm{~d} \mathcal{N}=(1,0)$ SCFTs with $G=\mathrm{SU}(3), \mathrm{SO}(8), E_{6}, E_{7}$. In this case, $\phi_{\lambda}\left(Q^{\vee}\right) \neq Q^{\vee}$; $\|\omega\|^{2} / 2$ for any $\omega \in \phi_{\lambda}\left(Q^{\vee}\right)$ and thus $d$ is not an integer. In fact we find

$$
\frac{\|\omega\|^{2}}{2}=\frac{\mathfrak{n}-2}{\mathfrak{n}}+k, \quad k \in \mathbb{Z}_{\geq 0}
$$

where the minimum norm square $(\mathfrak{n}-2) / \mathfrak{n}$ is reached if and only if $\omega$ is in a lowest dimensional irreducible representation ${ }^{24} \square_{G}$ of $G$. As a consequence, the right hand side of the elliptic blowup equations (3.4) vanishes

$$
\begin{aligned}
& \sum_{\omega \in \phi_{\lambda}\left(Q^{\vee}\right), d_{1,2} \in \mathbb{N}}^{\frac{1}{2}\|\omega\|^{2}+d_{1}+d_{2}=d}(-1)^{\left|\phi_{\lambda}^{-1}(\omega)\right|} \cdot \theta_{i}^{[a]}\left(\mathfrak{n} \tau,(\mathfrak{n}-2)\left(\epsilon_{1}+\epsilon_{2}\right)-\mathfrak{n}\left(\left(\frac{1}{2}|| \omega \|^{2}+d_{1}\right) \epsilon_{1}+\left(\frac{1}{2}|| \omega \|^{2}+d_{2}\right) \epsilon_{2}-m_{\omega}\right)\right) \\
& \quad \times A_{\omega}(m) \cdot \mathbb{E}_{d_{1}}\left(\tau, m-\epsilon_{1} \omega, \epsilon_{1}, \epsilon_{2}-\epsilon_{1}\right) \cdot \mathbb{E}_{d_{2}}\left(\tau, m-\epsilon_{2} \omega, \epsilon_{1}-\epsilon_{2}, \epsilon_{2}\right)=0, \quad \lambda \notin Q^{\vee} \text { fixed } d .
\end{aligned}
$$

Following the nomenclature of [15], we call these equations of the vanishing type.

The number of inequivalent embeddings $\phi_{\lambda}$ of this kind is $\left|P: Q^{\vee}\right|-1$, which happens to be the order of the automorphism group of the Dynkin diagram $\Gamma_{G}$ for

\footnotetext{
${ }^{24}$ This can be the fundamenal representation, the anti-fundamental representation, and in the case of $\mathrm{SO}(8)$ the two spinor representations.
} 
$G=\mathrm{SU}(3), \mathrm{SO}(8), E_{6}, E_{7}$. As representatives we can choose $\lambda$ to be a fundamental weight ${ }^{25}$ that generates $\square_{G}$ as the highest weight. The total number of inequivalent vanishing blowup equations for each of these models is $\mathfrak{n}\left(\left|P: Q^{\vee}\right|-1\right)$. Furthermore, using the property that the leading $Q_{\tau}$ order of $\mathbb{E}_{d}$ is $-d h_{G}^{\vee} / 6$, the equations (3.46) at the leading order requires that

$$
\sum_{\alpha \in \Delta^{+}}\left((\omega \cdot \alpha)^{2}-\left(\omega_{0} \cdot \alpha\right)^{2}\right)=2 k h_{G}^{\vee}, \quad \omega \in \phi_{\lambda}\left(Q^{\vee}\right), \omega_{0} \in \square_{G},
$$

where the integer $k$ is associated to the weight vector $\omega$ by (3.45), and it is again guaranteed by the Lie algebraic identity (A.5).

With the elliptic genus solved from the recursion formulas (3.32) plugged in, the vanishing blowup equations give rise to infinitely many nontrivial identities of Jacobi forms. In the lowest order, $d=(\mathfrak{n}-2) / \mathfrak{n}$ and $\phi_{\lambda}\left(Q^{\vee}\right)$ is chosen to be one of the lowest dimensional representations

$$
\sum_{\omega \in \square_{G}}(-1)^{\left|\phi_{\lambda}^{-1}(\omega)\right|} \theta_{i}^{[a]}\left(\mathfrak{n} \tau, \mathfrak{n} m_{\omega}\right) \prod_{\substack{\beta \in \Delta_{+} \\ \omega \cdot \beta= \pm 1}} \frac{1}{\theta_{1}\left(m_{\beta}\right)}=0 .
$$

This elegant formula specializing to $G=\mathrm{SU}(3)$ and $\mathrm{SO}(8)$ has been explicitly shown and checked in [25]. Here we further checked it for $E_{6}$ and $E_{7}$ for various characteristic $a$ to higher order of $Q_{\tau}$. For example, for $E_{6}$, the relevant representation is $\mathbf{2 7}$, with the weights encoded in the character

$$
\chi_{\mathbf{2 7}}^{E_{6}}=\sum_{i=1}^{27} \prod_{j=1}^{6} e^{m_{j} w_{i j}} .
$$

Then for arbitrary $a \in \mathbb{Z} / 6$, the following identity holds: ${ }^{26}$

$$
\sum_{i=1}^{27}(-1)^{\left|\phi_{\lambda}^{-1}\left(\omega_{i}\right)\right|} \theta_{3}^{[a]}\left(6 \tau, 6 m_{\omega_{i}}\right) \prod_{\substack{\beta \in \Delta_{+}\left(E_{6}\right) \\ \omega_{i} \cdot \beta= \pm 1}} \frac{1}{\theta_{1}\left(m_{\beta}\right)}=0 .
$$

Note there actually exist two $\mathbf{2 7}$ representations due to the symmetry of Dynkin diagram of $E_{6}$, i.e. $\left|\operatorname{Aut}\left(\Gamma_{E_{6}}\right)\right|=2$, both of them make (3.51) holds. This also explains why there are two copies of vanishing $r$ field for $E_{6}$ geometry, as we will see in next section in table 9 . For higher base degree of the vanishing blowup equations, one can also write down some more complicated identities like (3.48). We checked them for all $G=\mathrm{SU}(3), \mathrm{SO}(8), E_{6,7}$ in the setting of refined BPS expansion to very high orders.

\footnotetext{
${ }^{25}$ Not all the fundamental weights generate a lowest dimensional irreducible representation. For instance, the fundamental weight of $\mathrm{SO}(8)$ corresponding to the central node in the Dynkin diagram $\Gamma_{\mathrm{SO}(8)}$ generates the adjoint representation. The nodes associated to the $\square_{G}$-generating fundamental weights are permuted precisely by the automorphism group $\operatorname{Aut}\left(\Gamma_{G}\right)$.

${ }^{26}$ Let us write the notations in components in case of any misunderstanding.

$$
\left|\phi_{\lambda}^{-1}\left(\omega_{i}\right)\right|=\sum_{j, k=1}^{6}\left(C_{E_{6}}^{-1}\right)_{j k} \omega_{i j}, \quad m_{\omega_{i}}=\sum_{j, k=1}^{6} m_{k}\left(C_{E_{6}}^{-1}\right)_{j k} \omega_{i j}, \quad \omega_{i} \cdot \beta=\sum_{j=1}^{6} \omega_{i j} \beta_{j}, \quad m_{\beta}=\sum_{j=1}^{6} m_{j} \beta_{j},
$$
}

where $C_{E_{6}}$ is the Cartan matrix. 


\begin{tabular}{ccccc|}
\hline$G$ & $\mathrm{SU}(3)$ & $\mathrm{SO}(8)$ & $E_{6}$ & $E_{7}$ \\
\hline $\operatorname{dim}(\square)$ & 3 & 8 & 27 & 56 \\
$\operatorname{dim}\left(\Delta_{+}\right)$ & 3 & 12 & 36 & 63 \\
$\#\left\{\beta \in \Delta_{+}: \omega \cdot \beta=0, \forall \omega \in \square\right\}$ & 1 & 6 & 20 & 36 \\
$\#\left\{\beta \in \Delta_{+}: \omega \cdot \beta= \pm 1, \forall \omega \in \square\right\}$ & 2 & 6 & 16 & 27 \\
$\#\left\{\beta \in \Delta_{+}: \omega \cdot \beta= \pm 2, \forall \omega \in \square\right\}$ & 0 & 0 & 0 & 0 \\
\hline
\end{tabular}

Table 4. Distribution of positive roots with respect to product with weights in $\square_{G}$.

We list various Lie theoretical data including the distribution of positive roots with respect to product with any weight in $\square_{G}$ in table 4 , from which one can check (3.47) indeed holds.

\section{Elliptic genera for $6 \mathrm{~d}(1,0)$ minimal SCFTs}

In this section we illustrate explicitly the solution of one-string and two-string elliptic genera of minimal $6 \mathrm{~d}(1,0)$ SCFTs with $G=F_{4}, E_{6}, E_{7}, E_{8}$, using the elliptic blowup equations. The elliptic genera of the minimal theories with $G=\mathrm{SU}(3), \mathrm{SO}(8)$ have been computed in $[8,34]$, and we also reproduce some relevant results here. From these concrete results we summarise some universal features of the elliptic genera, including the expansion coefficients, the symmetric product approximation, and some additional symmetries. They are presented immediately in the first subsection, which one can then check in the following example subsections.

In this and the next sections, we work with the reduced elliptic genera which has the center-of-mass degree of freedom removed: ${ }^{27}$

$$
\mathbb{E}_{h_{G}^{(k)}}=\mathbb{E}_{\widetilde{h}_{G}^{(k)}} / \mathbb{E}_{\mathrm{c} . \mathrm{m} .},
$$

where

$$
\mathbb{E}_{\text {c.m. }}=-\frac{\theta_{1}\left(\epsilon_{1}\right) \theta_{1}\left(\epsilon_{2}\right)}{\eta^{2}} .
$$

In the reduced version, elliptic genera normally obtain simplification. For example, the reduced one-string elliptic genus is independent from $\epsilon_{-}$, i.e. $\mathrm{SU}(2)_{l}$, as expected.

\subsection{Universal behaviors of elliptic genera}

\subsubsection{Universal expansion}

For all possible gauge group $G$, recall $v \equiv \exp \left(\pi \mathrm{i}\left(\epsilon_{1}+\epsilon_{2}\right)\right)$ and $x \equiv \exp \left(\pi \mathrm{i}\left(\epsilon_{1}-\epsilon_{2}\right)\right)$, we propose the following general ansatz for the reduced $k$-string elliptic genera

$$
\mathbb{E}_{h_{G}^{(k)}}\left(v, x, Q_{\tau}, Q_{m_{i}}\right)=v^{k h_{G}^{\vee}-1} Q_{\tau}^{-\left(k h_{G}^{\vee}-1\right) / 6} \sum_{n=0}^{\infty} Q_{\tau}^{n} g_{k, G}^{(n)}\left(v, x, Q_{m_{i}}\right) .
$$

\footnotetext{
${ }^{27}$ We follow the notation of [37] where $\mathbb{E}_{\widetilde{h}_{G}^{(k)}}$ is used to stress it is the RR elliptic genus of underlying $2 \mathrm{~d}$ $(0,4)$ CFT associated to the $k$-strings in $6 \mathrm{~d}(1,0)$ minimal SCFT with gauge group $G$. It is the same with what we previously denoted as $\mathbb{E}_{k}$ to emphasize that it is coefficient of base degree $k$ in the topological string partition function.
} 
Here all $g_{k, G}^{(n)}\left(v, x, Q_{m_{i}}\right)$ are rational functions. In particular, $g_{1, G}^{(n)}$ is independent from $x$. One obvious symmetry for all $g_{k, G}^{(n)}$ is

$$
g_{k, G}^{(n)}\left(v, x, Q_{m_{i}}\right)=g_{k, G}^{(n)}\left(v, x^{-1}, Q_{m_{i}}\right),
$$

which comes from the symmetry between $\epsilon_{1}$ and $\epsilon_{2}$ in the Omega background, and can be understood as the Weyl symmetry of SU(2) $x_{x}$. From now on we use $\mathrm{SU}(2)_{x}$ to denote $\mathrm{SU}(2)_{l}$ symmetry to stress the associated fugacity is $x$. We can further compute the $v$-expansion of each $g_{k, G}^{(n)}$ function where the coefficients are finite sum of products between the characters of SU $(2)_{x}$ and characters of $G$ which respect Weyl symmetries of both groups. For example, $g_{k, G}^{(0)}=1+\ldots$ gives the Hilbert series of the reduced $k G$-instanton moduli space. In fact, we find plenty of universal coefficients for the first a few order $v$-expansion of $g_{k, G}^{(n)}$.

It is known that the Hilbert series of the reduced one-instanton moduli space for any simple gauge group $G$ has the expansion [41]

$$
g_{1, G}^{(0)}\left(v, Q_{m_{i}}\right)=\sum_{k=0}^{\infty} \chi_{n \theta}^{G} v^{2 n},
$$

where $\chi_{k \theta}$ is the character of the representation whose highest weight is $k$-multiple of the longest root $\theta$; in particular $\chi_{\theta}$ is the character of the adjoint representation of $G$. In particular this is true for $G=\mathrm{SU}(3), \mathrm{SO}(8), F_{4}, E_{6,7,8}$ when $g_{1, G}^{(0)}$ serves as the leading contribution to one-string elliptic genus. As for sub- and subsub-leading contributions, we find that except for $G=\mathrm{SU}(3)^{28}$

$g_{1, G}^{(1)}\left(v, Q_{m_{i}}\right)=1+\chi_{\theta}+\left(1+\chi_{\theta}+\chi_{2 \theta}+\chi_{\operatorname{Alt}^{2} \theta}\right) v^{2}+\left(2 \chi_{2 \theta}+\chi_{\operatorname{Alt}^{2} \theta}+\chi_{3 \theta}+B_{2}(G)\right) v^{4}+\mathcal{O}\left(v^{6}\right)$,

while except for $G=\mathrm{SU}(3), \mathrm{SO}(8)$,

$$
g_{1, G}^{(2)}\left(v, Q_{m_{i}}\right)=2+2 \chi_{\theta}+\chi_{\mathrm{Sym}^{2} \theta}+\mathcal{O}\left(v^{2}\right) .
$$

Here $B_{2}(G)$ are characters of some representations for which we do not find any universal expressions, and we list them in table $5 .{ }^{29}$ The exceptions of $\mathrm{SU}(3)$ and $\mathrm{SO}(8)$ can be explained by the higher structures of $\mathbb{E}_{1}$ revealed by its intriguing relation with the Schur indices of certain rank one 4d SCFTs discovered in [37], which we will review and extend in section 5 .

Furthermore, we find the Hilbert series of reduced two-instanton modulis space for any simple gauge group $G$ has the expansion

$$
\begin{aligned}
& g_{0, G}^{(2)}\left(v, x, Q_{m_{i}}\right)=1+\left(\chi_{\theta}+\chi_{3}\right) v^{2}+\chi_{\theta} \chi_{2} v^{3}+\left(\chi_{5}+\chi_{\theta} \chi_{3}+\chi_{\mathrm{Sym}^{2} \theta}\right) v^{4}+\left(\chi_{\theta} \chi_{4}+\left(\chi_{2 \theta}+\chi_{\mathrm{Alt}^{2} \theta}\right) \chi_{2}\right) v^{5} \\
& \quad+\left(\chi_{7}+\chi_{5} \chi_{\theta}+\chi_{3}\left(\chi_{\mathrm{Sym}^{2} \theta}+\chi_{2 \theta}\right)+\chi_{\mathrm{Sym}^{3} \theta}-C_{6}(G)\right) v^{6} \\
& \quad+\left(\chi_{\theta} \chi_{6}+\left(\chi_{2 \theta}+\chi_{\mathrm{Alt}^{2} \theta}\right) \chi_{4}+\left(\chi_{2 \theta}+\chi_{3 \theta}+\chi_{\mathrm{Alt}^{2} \theta}+B_{2}(G)+C_{7}(G)\right) \chi_{2}\right) v^{7} \\
& \quad+\left(\chi_{9}+\chi_{7} \chi_{\theta}+\chi_{5}\left(\chi_{\mathrm{Sym}^{2} \theta}+\chi_{2 \theta}\right)+\chi_{3}\left(\chi_{3 \theta}+\chi_{2 \theta}+B_{2}(G)+\chi_{\mathrm{Sym}^{3} \theta}-C_{6}(G)\right)+\chi_{\mathrm{Sym}^{4} \theta}-C_{8}(G)\right) v^{8} \\
& \quad+\left(\chi_{\theta} \chi_{8}+\left(\chi_{2 \theta}+\chi_{\mathrm{Alt}^{2} \theta}\right) \chi_{6}+\left(\chi_{2 \theta}+2 \chi_{3 \theta}+\chi_{\mathrm{Alt}^{2} \theta}+B_{2}(G)+C_{7}(G)\right) \chi_{4}+\ldots\right) v^{9}+\ldots
\end{aligned}
$$

\footnotetext{
${ }^{28}$ From now on, to shorten formulas, we do not explicitly write $G$ in each character.

${ }^{29}$ The bold numbers mean the character of representations with dimension of such number. Note different representations can have the same dimension sometimes, for instance, the representations $\mathbf{3 5}{ }_{v}, \mathbf{3 5}_{s}$ and $\mathbf{3 5}{ }_{c}$ of $\mathrm{SO}(8)$. To lighten the notation, we do not distinguish them in the table. Nevertheless, they can be recovered by taking into account the symmetry of Dynkin diagrams.
} 


\begin{tabular}{|c|c|c|c|c|c|c|c|}
\hline$G$ & $\chi_{\theta}$ & $\chi_{2 \theta}$ & $\chi_{3 \theta}$ & $B_{2}$ & $C_{6}$ & $C_{7}$ & $C_{8}$ \\
\hline$A_{2}$ & 8 & 27 & 64 & $2 \cdot 35$ & 1 & 27 & 8 \\
\hline$D_{4}$ & 28 & 300 & 1925 & 4096 & $2 \cdot \mathbf{2 8}$ & $3 \cdot 567$ & $2 \cdot(\mathbf{3 0 0}+\mathbf{3 5 0}+\mathbf{1})+3 \cdot \mathbf{3 5}$ \\
\hline$F_{4}$ & 52 & 1053 & 12376 & 29172 & 273 & 10829 & $8424+4096+324+26$ \\
\hline$E_{6}$ & 78 & 2430 & 43758 & 105600 & 650 & 34749 & $34749+2 \cdot 5824+650+78$ \\
\hline$E_{7}$ & 133 & 7371 & 238602 & 573440 & 1463 & 152152 & $150822+40755+1539$ \\
\hline$E_{8}$ & 248 & 27000 & 1763125 & 4096000 & 0 & 779247 & 147250 \\
\hline
\end{tabular}

Table 5. Certain representations appearing in the expansion of $g_{k, G}^{(n)}$ functions.

Here $\chi_{n}$ is the character of $n$-dimensional representation of $\mathrm{SU}(2)_{x}$. The expansion coefficients up to $v^{6}$ were already observed in [30], and we further push the observation up to $v^{8}$. We have checked this expression to be consistent with all the results on Hilbert series of reduced two $G$ instanton moduli space in [42]. In particular it is true for $G=\mathrm{SU}(3), \mathrm{SO}(8), F_{4}, E_{6,7,8}$ when $g_{2, G}^{(0)}$ is the leading contribution to the two-string elliptic genera. Note that in this expression, $C_{6}(G), C_{7}(G)$ are characters of certain representations of $G$ for which universal expressions are not found. They are collected for individual $G$ in table 5. As for the subleading and subsubleading contribution to the two-string elliptic genera, we find there exists the following universal $v$-expansion: except for $G=\mathrm{SU}(3)$,

$$
\begin{aligned}
g_{1, G}^{(2)}\left(v, x, Q_{m_{i}}\right)= & 1+\chi_{\theta}+\chi_{3}+\left(\chi_{\theta}+1\right) \chi_{2} v+\left(\chi_{5}+\left(2 \chi_{\theta}+3\right) \chi_{3}+\left(\chi_{\theta}+1\right)^{2}\right) v^{2} \\
& +\left(\left(2 \chi_{\theta}+1\right) \chi_{4}+\left(\chi_{2 \theta}+\left(\chi_{\theta}+1\right)^{2}+\chi_{\mathrm{Sym}^{2} \theta}\right) \chi_{2}\right) v^{3} \\
& +\left(\chi_{7}+\left(2 \chi_{\theta}+3\right) \chi_{5}+\ldots\right) v^{4}+\mathcal{O}\left(v^{5}\right)
\end{aligned}
$$

while except for $G=\mathrm{SU}(3)$ and $\mathrm{SO}(8)$,

$$
\begin{aligned}
g_{2, G}^{(2)}\left(v, x, Q_{m_{i}}\right)= & \left(\chi_{5}+\left(\chi_{\theta}+2\right) \chi_{3}+\chi_{\mathrm{Sym}^{2} \theta}+2 \chi_{\theta}+4\right)+\left(\left(\chi_{\theta}+1\right) \chi_{4}+\left(\left(\chi_{\theta}+1\right)^{2}+2\left(\chi_{\theta}+1\right)\right) \chi_{2}\right) v \\
& +\left(\chi_{7}+\left(2 \chi_{\theta}+4\right) \chi_{5}+\left(\left(\chi_{\theta}+1\right)^{2}+2 \chi_{\mathrm{Sym}^{2} \theta}+6 \chi_{\theta}+9\right) \chi_{3}+\ldots\right) v^{2}+\mathcal{O}\left(v^{3}\right) .
\end{aligned}
$$

For the reduced three string elliptic genus $\mathbb{E}_{h_{G}^{(3)}}$, although we have not checked for all six $G$ due to the complexity of computation, still we propose the following universal expansion:

$$
\begin{aligned}
g_{0, G}^{(3)}\left(v, x, Q_{m_{i}}\right)= & 1+\left(\chi_{3}+\chi_{\theta}\right) v^{2}+\left(\chi_{4}+\chi_{\theta} \chi_{2}\right) v^{3}+\left(\chi_{5}+\chi_{\theta} \chi_{3}+\chi_{\mathrm{Sym}^{2} \theta}+1\right) v^{4} \\
& +\left(\chi_{6}+\left(2 \chi_{\theta}+1\right) \chi_{4}+2 \chi_{\mathrm{Sym}^{2} \theta}\right) v^{5} \\
& +\left(2 \chi_{7}+3 \chi_{\theta} \chi_{5}+\left(\chi_{2 \theta}+3 \chi_{\mathrm{Sym}^{2} \theta}+1\right) \chi_{3}+\chi_{\mathrm{Sym}^{3} \theta}+\chi_{\mathrm{Sym}^{2} \theta}\right) v^{6}+\mathcal{O}\left(v^{7}\right)
\end{aligned}
$$

We have checked this against the three-instanton Hilbert series for $\mathrm{SU}(2), G_{2}, \mathrm{SO}(7), \mathrm{Sp}(4)$, $\mathrm{Sp}(6)$ in $[43,60]$, and against the three-string elliptic genus for $\mathrm{SU}(3)$ [34]. Note the first 
two terms also agree with the rank three $E_{6}$ Hall-Littlewood index ((A.14) in [45]). For the subleading $Q_{\tau}$ order, again except $\mathrm{SU}(3)$, we propose

$$
\begin{aligned}
g_{1, G}^{(3)}\left(v, x, Q_{m_{i}}\right)= & \left(\chi_{3}+\chi_{\theta}+1\right)+\left(\chi_{4}+\left(\chi_{\theta}+1\right) \chi_{2}\right) v \\
& +\left(\chi_{5}+\left(3 \chi_{\theta}+4\right) \chi_{3}+2 \chi_{\mathrm{Sym}^{2} \theta}+\chi_{\theta}+2\right) v^{2}+\mathcal{O}\left(v^{3}\right) .
\end{aligned}
$$

As in rank one and two cases, for $\mathrm{SU}(3)$, the higher contributions begin to merge in at $Q_{\tau}$ subleading order.

All above $v$-expansion coefficients can be easily obtained by setting $Q_{m}=1$ in $g_{n, G}^{(k)}\left(v, x, Q_{m}\right)$. Thus the rational functions $g_{n, G}^{(k)}(v, x, 1)$ are very useful as they encode most information. For large $k$ or $n$, such rational functions with generic $x$ turn out to be too lengthy. One can take the unrefined limit $x=1$ in $g_{n, G}^{(k)}(v, x, 1)$ to still store meaning information on arbitrary order coefficients of $v$-expansion. Indeed, when the fugacities of flavor as well as $\mathrm{SU}(2)_{x}$ are turned off, we find

$$
\begin{aligned}
& g_{1, G}^{(n)}(v)=\frac{1}{\left(1-v^{2}\right)^{2\left(h_{G}^{\vee}-1\right)}} \times P_{1, G}^{(n)}(v), \\
& g_{2, G}^{(n)}(v)=\frac{1}{\left(1-v^{2}\right)^{2\left(h_{G}^{\vee}-1\right)}(1+v)^{2 b_{G}}\left(1+v+v^{2}\right)^{2 h_{G}^{\vee}-1}} \times P_{2, G}^{(n)}(v) .
\end{aligned}
$$

The exponents $b_{G}$ are given by

\begin{tabular}{|ccccccc|}
\hline$G$ & $\mathrm{SU}(3)$ & $\mathrm{SO}(8)$ & $F_{4}$ & $E_{6}$ & $E_{7}$ & $E_{8}$ \\
\hline$b$ & 3 & 6 & 11 & 16 & 26 & 46 \\
\hline
\end{tabular}

We notice that $b=5 h_{G}^{\vee} / 3-4$ except for $\mathrm{SU}(3)$. The numerators $P_{1, G}^{(n)}(v)$ and $P_{2, G}^{(n)}(v)$ are palindromic Laurent polynomials. They have negative powers of $v$ when $n$ is large. Nevertheless $P_{1, G}^{(0)}(v), P_{2, G}^{(0)}(v)$ are both polynomials and their maximum degrees are $h_{G}^{\vee}-1$ and $2\left(2 h_{G}^{\vee}-1\right)+2 b_{G}$ respectively. The explicit expressions of $P_{k, G}^{(n)}(v)$ for the minimal SCFTs with $G=\mathrm{SU}(3), \mathrm{SO}(8), F_{4}, E_{6}, E_{7}, E_{8}$ are presented in the following example subsections and also appendix F.

\subsubsection{Symmetric product approximation}

It was noticed both in [42] and [45] that the reduced two $G$-instanton Hilbert series can be realized as certain symmetric product of two one $G$-instantons as approximation:

$$
\frac{1}{1-v x^{ \pm 1}} g_{0, G}^{(2)}(v, x, a)=\frac{1}{2}\left(\left(g_{0, G}^{(1)}(v, a) \frac{1}{1-v x^{ \pm 1}}\right)^{2}+g_{0, G}^{(1)}\left(v^{2}, a^{2}\right) \frac{1}{1-v^{2} x^{ \pm 2}}\right)+\mathcal{O}\left(v^{4}\right) .
$$

Here we adopt their notation $a=Q_{m_{G}}$ to lighten the notation. It also was noticed in [43] that the reduced three $G$-instanton Hilbert series can be realized as certain symmetric product of three one $G$-instantons as approximation:

$$
\begin{aligned}
\frac{1}{1-v x^{ \pm 1}} g_{0, G}^{(3)}(v, x, a)= & \frac{1}{6}\left(\left(\frac{1}{1-v x^{ \pm 1}} g_{0, G}^{(1)}(v, a)\right)^{3}+\frac{3}{\left(1-v x^{ \pm 1}\right)\left(1-v^{2} x^{ \pm 2}\right)} g_{0, G}^{(1)}(v, a) g_{0, G}^{(1)}\left(v^{2}, a^{2}\right)\right. \\
& \left.+\frac{2}{1-v^{3} x^{ \pm 3}} g_{0, G}^{(1)}\left(v^{3}, a^{3}\right)\right)+\mathcal{O}\left(v^{4}\right)
\end{aligned}
$$


The above formulas have clear physical meaning. For example in (4.16), the first term represents the configuration where three instantons are far from each other, the second term represents the configuration where two instantons sit on the same site and the third one is far from them, while the third term represents the configuration where all three instantons sit on the same site. Note the triple symmetric product would give the coefficient of $v^{4}$ of $g_{0, G}^{(3)}$ as $\chi_{5}+\chi_{\theta} \chi_{3}+\chi_{2 \theta}+\chi_{\mathrm{Sym}^{2} \theta}+1$, one can see the difference with (4.11) begins to appear.

In fact, it is reasonable that arbitrary $k G$-instanton Hilbert series can be realized as symmetric product of $k G$-instantons as approximation:

$$
\frac{1}{1-v x^{ \pm 1}} g_{0, G}^{(k)}(v, x, a)=\operatorname{Sym}_{\mathcal{M}_{G, 1}}^{k}(v, x, a)+\mathcal{O}\left(v^{4}\right)
$$

where $\operatorname{Sym}_{G, 1}^{k}(v, x, a)$ can be obtained from generating function

$$
\sum_{k=1}^{\infty} \operatorname{Sym}_{\mathcal{M}_{G, 1}}^{k}(v, x, a) Q^{k}=\operatorname{PE}\left[\frac{Q}{1-v x^{ \pm 1}} g_{0, G}^{(1)}(v, a)\right] \equiv \operatorname{PE}\left[\widetilde{g}_{0, G}^{(1)}(v, x, a) Q\right] .
$$

For example,

$$
\begin{gathered}
\operatorname{Sym}_{\mathcal{M}_{G, 1}}^{4}(v, x, a)=\frac{1}{24}\left(\left(\widetilde{g}_{0, G}^{(1)}(v, x, a)\right)^{4}+6\left(\widetilde{g}_{0, G}^{(1)}(v, x, a)\right)^{2} \widetilde{g}_{0, G}^{(1)}\left(v^{2}, x^{2}, a^{2}\right)+3\left(\widetilde{g}_{0, G}^{(1)}\left(v^{2}, x^{2}, a^{2}\right)\right)^{2}\right. \\
\left.\quad+8 \widetilde{g}_{0, G}^{(1)}(v, x, a) \widetilde{g}_{0, G}^{(1)}\left(v^{3}, x^{3}, a^{3}\right)+6 \widetilde{g}_{0, G}^{(1)}\left(v^{4}, x^{4}, a^{4}\right)\right) \\
=1+\left(\chi_{3}+\chi_{\theta}\right) v^{2}+\left(\chi_{4}+\chi_{\theta} \chi_{2}\right) v^{3}+\mathcal{O}\left(v^{4}\right) .
\end{gathered}
$$

It is not hard to find that for all $k \geq 3$, the leading coefficients in $v$ expansion of symmetric product are the same:

$$
\begin{aligned}
\operatorname{Sym}_{\mathcal{M}_{G, 1}}^{k}(v, x, a) & =\frac{1}{k !}\left(\left(\widetilde{g}_{0, G}^{(1)}(v, x, a)\right)^{k}+C_{k}^{2}\left(\widetilde{g}_{0, G}^{(1)}(v, x, a)\right)^{k-2} \widetilde{g}_{0, G}^{(1)}\left(a^{2}, v^{2}, x^{2}\right)+\ldots\right) \\
& =1+\left(\chi_{3}+\chi_{\theta}\right) v^{2}+\left(\chi_{4}+\chi_{\theta} \chi_{2}\right) v^{3}+\mathcal{O}\left(v^{4}\right) .
\end{aligned}
$$

Here the first term represents all $k$ instantons are far from each other, while the second term represents two instantons sit at the same site and the rest $k-2$ instanton are far from them and each other... From $v^{4}$, the interaction among instantons will contribute.

We can also include $g_{0, G}^{(k)}$ into the elliptic genus to write down the above symmetric product approximation. For example in the reduced three-string elliptic genus, since

$$
\mathbb{E}_{\widetilde{h}_{G}^{(3)}}\left(v, x, a, Q_{\tau}\right)=\frac{v^{3 h_{G}^{\vee}}}{1-v x^{ \pm 1}} g_{0, G}^{(3)}(v, x, a) Q_{\tau}^{-h_{G}^{\vee} / 2}+\mathcal{O}\left(Q_{\tau}^{-h_{G}^{\vee} / 2+1}\right),
$$

combining (4.16), we obtain

$$
\begin{aligned}
\mathbb{E}_{\widetilde{h}_{G}^{(3)}}\left(v, x, a, Q_{\tau}\right)=\frac{1}{6}( & \mathbb{E}_{\widetilde{h}_{G}^{(1)}}\left(v, x, a, Q_{\tau}\right)^{3}+3 \mathbb{E}_{\widetilde{h}_{G}^{(1)}}\left(v, x, a, Q_{\tau}\right) \mathbb{E}_{\widetilde{h}_{G}^{(1)}}\left(v^{2}, x^{2}, a^{2}, Q_{\tau}^{2}\right) \\
& \left.+2 \mathbb{E}_{\widetilde{h}_{G}^{(1)}}\left(v^{3}, x^{3}, a^{3}, Q_{\tau}^{3}\right)\right)+\ldots,
\end{aligned}
$$

which holds for the leading $Q_{\tau}$ order and the first four $v$-expansion coefficients. For arbitrary $k$-strings elliptic genus, it is better to use Hecke transformation. Neglecting the 
interaction among strings, the resulting $k$-strings elliptic genus $\mathbb{E}_{\operatorname{Sym}_{G}^{(k)}}\left(v, x, a, Q_{\tau}\right)$ can be generated from

$$
\sum_{k=0} \mathbb{E}_{\operatorname{Sym}_{G}^{(k)}}\left(v, x, a, Q_{\tau}\right) Q^{k}=\exp \left[\sum_{n \geq 0} Q^{n} \frac{1}{n} \sum_{\substack{c d=n \\ c, d>0}} \sum_{b(\bmod d)} \mathbb{E}_{\widetilde{h}_{G}^{(1)}}\left(\frac{c \tau+b}{d}, c \epsilon_{i}, c m_{G}\right)\right] .
$$

Note this relies on the Jacobi form nature of $\mathbb{E}_{\widetilde{h}_{G}^{(1)}}\left(\tau, \epsilon_{i}, m_{G}\right)$. Also take $d=1$ in (4.23), one will go back to instanton formula (4.18) where there is no modularity. Finally, we obtain

$$
\mathbb{E}_{\widetilde{h}_{G}^{(k)}}\left(v, x, a, Q_{\tau}\right)=\mathbb{E}_{\operatorname{Sym}_{G}^{(k)}}\left(v, x, a, Q_{\tau}\right)+\mathcal{O}\left(Q_{\tau}^{-k h_{G}^{\vee} / 6+1}\right)+\mathcal{O}\left(v^{k h_{G}^{\vee}+4}\right) .
$$

As we have checked this symmetric product approximation does not give exact subleading $Q_{\tau}$ orders $g_{1, G}^{(k)}$ even for its leading $v$-expansion coefficient. This means all subleading $Q_{\tau}$ orders involves interaction among strings.

\subsubsection{Symmetries}

Besides the obvious symmetry

$$
\mathbb{E}_{h_{G}^{(k)}}\left(v, x, Q_{\tau}, Q_{m}\right)=\mathbb{E}_{h_{G}^{(k)}}\left(v, x^{-1}, Q_{\tau}, Q_{m}\right),
$$

which comes from the symmetry between $\epsilon_{1}$ and $\epsilon_{2}$ in Omega background, it was found in [37] that the reduced one-string elliptic genus $\mathbb{E}_{h_{G}^{(1)}}\left(v, Q_{\tau}\right)$ satisfies an additional symmetry

$$
\mathbb{E}_{h_{G}^{(1)}}\left(Q_{\tau}^{1 / 2} / v, Q_{\tau}\right)=(-1)^{\mathfrak{n}+1} v^{2\left(1-h_{G}^{\vee} / 3\right)} Q_{\tau}^{\left(h_{G}^{\vee} / 3-1\right) / 2} \mathbb{E}_{h_{G}^{(1)}}\left(v, Q_{\tau}\right) .
$$

Here the dependence on $m_{G}$ is implicit. This symmetry was later interpreted as a spectral flow symmetry in [38]. The left hand side of (4.26) actually computes the NS-R elliptic genus, which should be equal to the R-R elliptic genus on the right hand side due to the lack of chiral fermions in the minimal SCFT in consideration. See section 6.4 of [37] for a detailed discussion.

We extend the symmetry (4.26) to arbitrary $k$-string elliptic genus $\mathbb{E}_{h_{G}^{(k)}}\left(v, x, Q_{\tau}\right)$ :

$$
\mathbb{E}_{h_{G}^{(k)}}\left(\frac{Q_{\tau}^{1 / 2}}{v}, \frac{Q_{\tau}^{1 / 2}}{x}, Q_{\tau}\right)=(-1)^{\mathfrak{n} k+1} v^{\frac{k(k-5)}{6} h_{G}^{\vee}+k^{2}+1} x^{-\frac{k(k-1)}{6} h_{G}^{\vee}-k^{2}+1} Q_{\tau}^{\left(k h_{G}^{\vee}-3\right) / 6} \mathbb{E}_{h_{G}^{(k)}}\left(v, x, Q_{\tau}\right),
$$

which can be derived by combining (3.25) and the modular anomaly of elliptic genera (3.12). For the situation where 2 d quiver description is known, i.e. $G=\mathrm{SU}(3)$ and $\mathrm{SO}(8)$, the above symmetry can also be obtained by looking into the transformation of integrand of localization with the quasi-periodicity of Jacobi theta function (D.5), (D.6). Note symmetry (4.27) is a nonperturbative symmetry, which can not be seen from the $Q_{\tau}$ expansion of the elliptic genus, except for the one-string case that is (4.26). ${ }^{30}$ This means (4.27) should be seen as

\footnotetext{
${ }^{30}$ Practically, we find that for the two-string elliptic genus, when $Q_{\tau}$ order is enough high, for one order of $Q_{\tau}$ goes up, the leading $v$ order goes down for 3. Thus, if one naively does the transformation for the left hand side of (4.27) in $Q_{\tau}$ expansion, one would get infinite negative order of $Q_{\tau}$. Similar situation also happens for three-string elliptic genus. But for one-string elliptic genus, luckily for one order of $Q_{\tau}$ goes up, the leading $v$ order goes down for 2 , which only result in finite negative order of $Q_{\tau}$.
} 


\begin{tabular}{|cccc|}
\hline \multirow{4}{*}{ unity } & $r$ & old $r$ & fundamental weights \\
\hline \multirow{4}{*}{ vanishing } & $(0,0,0,1)$ & $(0,0,0,1)$ & \\
& $(0,0,0,3)$ & $(0,0,0,3)$ & $\omega_{1}+\omega_{2}$ \\
& $(0,0,0,5)$ & $(0,0,0,-1)$ & \\
& $(-2,2,0,1)$ & $(-2,2,0,1)$ & \\
& $(-2,2,0,5)$ & $(0,-2,2,1)$ & $\omega_{1}$ \\
vanishing & $(-2,0,2,1)$ & $(-2,0,2,1)$ & \\
& $(-2,0,2,3)$ & $(0,2,-2,1)$ & $\omega_{2}$ \\
& $(-2,0,2,5)$ & $(2,-2,0,1)$ & \\
\hline
\end{tabular}

Table 6. The $r$-fields of the $\mathfrak{n}=3$ model and the fundamental weights of $\mathfrak{a}_{2}$ which induce the embedding $\phi: Q^{\vee} \hookrightarrow P$. The old $r$-fields are from our previous paper [25]. They are equivalent with the $r$ in the second column by $2 C \cdot \underline{n}$ shift.

the symmetry of the chiral algebra associated to the underlying $(0,4) 2 \mathrm{~d}$ CFT, as suggested in $[37]$.

\subsection{Revisiting $G=\mathrm{SU}(3)$ and $\mathrm{SO}(8)$}

With the new understanding on the structure of $r$ fields of $6 \mathrm{~d}(1,0)$ minimal SCFTs for all $G$, we now can reproduce all $r$ fields for $\mathrm{SU}(3)$ and $\mathrm{SO}(8)$ found in [25] using just the fundamental weights of the Lie algebras. We summarize the correspondence between the $r$ fields given in [25] and fundamental weights in tables 6 and 7 .

The elliptic genera of $6 \mathrm{~d}(1,0)$ SCFT with $G=\mathrm{SU}(3)$ were computed using JeffreyKirwan residue in [34], and were checked to satisfy the elliptic blowup equations [25]. Following the general proposal (4.3), the reduced two-string elliptic genus for SU(3) model can be written as

$$
\mathbb{E}_{h_{A_{2}}^{(2)}}\left(v, x, Q_{\tau}, m_{i}\right)=v^{5} Q_{\tau}^{-5 / 6} \sum_{n=0}^{\infty} Q_{\tau}^{n} g_{2, A_{2}}^{(n)}\left(v, x, Q_{m_{i}}\right),
$$

where $g_{2, A_{2}}^{(n)}\left(v, x, Q_{m_{i}}\right)$ are rational functions. We computed $g_{2, A_{2}}^{(n)}\left(v, x, Q_{m_{i}}=1\right)$ up to $n=10$. Let us turn off the fugacities of both $\mathrm{SU}(3)$ and $\mathrm{SU}(2)_{x}$, we obtain

$$
g_{2, A_{2}}^{(n)}\left(v, x=1, Q_{m_{i}}=1\right)=\frac{1}{(1-v)^{10}(1+v)^{6}\left(1+v+v^{2}\right)^{5}} \times P_{2, A_{2}}^{(n)}(v),
$$

where all $P_{2, A_{2}}^{(n)}(v)$ are palindromic Laurent polynomials, in which only $P_{2, A_{2}}^{(0)}(v)$ is a true polynomial:

$$
P_{2, A_{2}}^{(0)}(v)=1+v+6 v^{2}+17 v^{3}+31 v^{4}+52 v^{5}+92 v^{6}+110 v^{7}+112 v^{8}+110 v^{9}+\cdots+v^{16} .
$$

Here the ellipsis is completed by making the expression palindromic. This agrees with the Hilbert series of reduced two SU(3)-instanton moduli space in [42]. For the subleading 


\begin{tabular}{|cccc|}
\hline \multirow{4}{*}{ unity } & $r$ & old $r$ & fundamental weights \\
\hline \multirow{4}{*}{ vanishing } & $(0,0,0,0,0,0)$ & $(0,0,0,0,0,0)$ & \\
& $(0,0,0,0,0,2)$ & $(0,0,0,0,0,2)$ & $\omega_{c}$ \\
& $(0,0,0,0,0,4)$ & $(0,0,0,0,0,4)$ & \\
& $(-2,0,0,0,0,6)$ & $(0,0,0,0,0,-2)$ & \\
& $(-2,2,0,0,0,0)$ & $(-2,2,0,0,0,0)$ & \\
& $(-2,2,0,0,0,4)$ & $(-2,-2,0,0,2,2)$ & $\omega_{1}$ \\
vanishing & $(-2,2,0,0,0,6)$ & $(0,0,-2,-2,2,2,2)$ & \\
& $(-2,0,2,0,0,0)$ & $(-2,0,2,0,0,0)$ & \\
& $(-2,0,2,0,0,2)$ & $(-2,0,2,0,2,2)$ & $\omega_{2}$ \\
& $(-2,0,2,0,0,4)$ & $(0,-2,0,2,0,0)$ & \\
vanishing & $(-2,0,2,0,0,6)$ & $(0,-2,0,-2,2,2)$ & \\
& $(-2,0,0,2,0,0)$ & $(-2,0,0,2,0,0)$ & \\
& $(-2,0,0,2,0,2)$ & $(-2,0,0,-2,2,2)$ & $\omega_{3}$ \\
& $(-2,0,0,2,0,6)$ & $(0,-2,-2,0,2,0)$ & \\
\hline
\end{tabular}

Table 7. The $r$-fields of the $\mathfrak{n}=4$ model and the fundamental weights of $\mathfrak{d}_{4}$ which induce the embedding $\phi: Q^{\vee} \hookrightarrow P$. The old $r$-fields are from our previous paper [25]. They are equivalent with the $r$ in the second column by $2 C \cdot \underline{n}$ shift.

order, $P_{2, A_{2}}^{(1)}(v)$ starts with negative power of $v$, which is different from all the other minimal SCFTs. ${ }^{31}$ Indeed,

$$
\begin{gathered}
P_{2, A_{2}}^{(1)}(v)=v^{-4}\left(1+3 v+8 v^{2}+11 v^{3}+18 v^{4}+13 v^{5}+55 v^{6}+238 v^{7}+601 v^{8}+1121 v^{9}+1777 v^{10}\right. \\
\left.+2262 v^{11}+2424 v^{12}+2262 v^{13}+\cdots+v^{24}\right) .
\end{gathered}
$$

More results on $P_{2, A_{2}}^{(n)}(v)$ with $n>1$ can be found in appendix F. Let us also show some results with generic fugacities, for example,

$$
\begin{aligned}
g_{0, A_{2}}^{(2)}\left(v, x, m_{i}\right)= & 1+\left(\mathbf{8}+\chi_{3}\right) v^{2}+\mathbf{8} \chi_{2} v^{3}+\left(\chi_{5}+\mathbf{8} \chi_{3}+\operatorname{Sym}^{2} \mathbf{8}\right) v^{4}+\left(\mathbf{8} \chi_{4}+\left(\mathbf{2 7}+\operatorname{Alt}^{2} \mathbf{8}\right) \chi_{2}\right) v^{5} \\
& +\left(\chi_{7}+\mathbf{8} \chi_{5}+\left(\operatorname{Sym}^{2} \mathbf{8}+\mathbf{2 7}\right) \chi_{3}+\operatorname{Sym}^{3} \mathbf{8}-\mathbf{1}\right) v^{6}+\mathcal{O}\left(v^{7}\right), \\
v^{4} g_{1, A_{2}}^{(2)}\left(v, x, m_{i}\right)= & 1+\chi_{2} v+\left(\chi_{3}+\mathbf{8}\right) v^{2}+\left(\chi_{4}+\mathbf{8} \chi_{2}\right) v^{3}+\left(\chi_{5}+(\mathbf{8}+\mathbf{1}) \chi_{3}+\operatorname{Sym}^{2} \mathbf{8}\right) v^{4} \\
& +\left(\chi_{6}+\mathbf{8} \chi_{4}+\operatorname{Sym}^{2} \mathbf{8} \chi_{2}\right) v^{5}+\mathcal{O}\left(v^{6}\right) .
\end{aligned}
$$

Note (4.32) agrees with our universal expansion formula (4.8).

\footnotetext{
${ }^{31}$ This phenomenon as also occurring in one-string elliptic genus, will be discussed in detail in section 5 .
} 
Similarly, the reduced three-string elliptic genus for $\mathrm{SU}(3)$ model can be written as

$$
\mathbb{E}_{h_{A_{2}}^{(2)}}\left(v, x, Q_{\tau}, m_{i}\right)=v^{8} Q_{\tau}^{-4 / 3} \sum_{n=0}^{\infty} Q_{\tau}^{n} g_{3, A_{2}}^{(n)}\left(v, x, Q_{m_{i}}\right),
$$

where all $g_{3, A_{2}}^{(n)}\left(v, x, Q_{m_{i}}\right)$ are rational functions. We computed $g_{3, A_{2}}^{(n)}\left(v, x, Q_{m_{i}}=1\right)$ up to $n=6$. Turning off the fugacities of both $\mathrm{SU}(3)$ and $\mathrm{SU}(2)_{x}$, we obtain

$$
g_{3, A_{2}}^{(n)}\left(v, x=1, Q_{m_{i}}=1\right)=\frac{1}{(1-v)^{16}(1+v)^{10}\left(1+v^{2}\right)^{5}\left(1+v+v^{2}\right)^{6}} \times P_{3, A_{2}}^{(n)}(v),
$$

where all $P_{3, A_{2}}^{(n)}(v)$ are palindromic Laurent polynomials, in which only $P_{3, A_{2}}^{(0)}(v)$ is a true polynomial:

$$
\begin{aligned}
P_{3, A_{2}}^{(0)}(v)= & 1+6 v^{2}+14 v^{3}+40 v^{4}+82 v^{5}+213 v^{6}+388 v^{7}+772 v^{8}+1260 v^{9}+2079 v^{10}+2986 v^{11} \\
& +4226 v^{12}+5226 v^{13}+6384 v^{14}+6940 v^{15}+7334 v^{16}+6940 v^{17}+\ldots+v^{32}, \\
P_{3, A_{2}}^{(1)}(v)= & v^{-4}\left(1+v^{2}\right)\left(1+2 v+8 v^{2}+24 v^{3}+62 v^{4}+114 v^{5}+242 v^{6}+456 v^{7}+964 v^{8}+1926 v^{9}\right. \\
& +4225 v^{10}+8448 v^{11}+16317 v^{12}+28038 v^{13}+44954 v^{14}+64960 v^{15}+87437 v^{16} \\
& \left.+106636 v^{17}+121046 v^{18}+125368 v^{19}+121046 v^{20}+\ldots+v^{38}\right) .
\end{aligned}
$$

Note $g_{3, A_{2}}^{(0)}$ agrees with our universal expansion formula (4.11). More higher $P_{3, A_{2}}^{(n)}(v)$ can be found in appendix F.

The elliptic genera for the $6 \mathrm{~d}(1,0)$ SCFT with $G=\mathrm{SO}(8)$ were computed using Jeffrey-Kirwan residue in [24], and they were checked to satisfy elliptic blowup equations in [25]. Let us write the reduced two-string elliptic genus as

$$
\mathbb{E}_{h_{D_{4}}^{(2)}}\left(v, x, Q_{\tau}, m_{i}\right)=v^{11} Q_{\tau}^{-11 / 6} \sum_{n=0}^{\infty} Q_{\tau}^{n} g_{2, D_{4}}^{(n)}\left(v, x, m_{i}\right) .
$$

We computed $g_{n, D_{4}}^{(2)}\left(v, x, m_{i}=0\right)$ up to $n=6$. In particular, $g_{n, D_{4}}^{(2)}$ for $n=0,1,2$ agree with our universal expansion formulas (4.8), (4.9). Turning off the $\mathrm{SU}(2)_{x}$ fugacity, we have

$$
g_{2, D_{4}}^{(n)}\left(v, x=1, m_{i}=0\right)=\frac{1}{(1-v)^{22}(1+v)^{12}\left(1+v+v^{2}\right)^{11}} \times P_{2, D_{4}}^{(n)}(v) .
$$

Here $P_{2, D_{4}}^{(n)}(v)$ are palindromic Laurent polynomials. In particular, only for $n=0,1$, they are true polynomials:

$$
\begin{aligned}
P_{2, D_{4}}^{(0)}(v)= & 1+v+20 v^{2}+65 v^{3}+254 v^{4}+841 v^{5}+2435 v^{6}+6116 v^{7}+14290 v^{8}+29700 v^{9} \\
& +55947 v^{10}+96519 v^{11}+152749 v^{12}+220408 v^{13}+293226 v^{14}+359742 v^{15} \\
& +406014 v^{16}+421960 v^{17}+406014 v^{18}+\cdots+v^{34} . \\
P_{2, D_{4}}^{(1)}(v)= & \left(1+v^{2}\right)\left(32+90 v+697 v^{2}+2913 v^{3}+10582 v^{4}+34415 v^{5}+97961 v^{6}+242492 v^{7}\right. \\
& +540749 v^{8}+1085137 v^{9}+1958185 v^{10}+3205774 v^{11}+4789888 v^{12}+6522178 v^{13} \\
& \left.+8110633 v^{14}+9248825 v^{15}+9668450 v^{16}+9248825 v^{17}+\cdots+v^{32}\right) .
\end{aligned}
$$

Note the above $g_{0, D_{4}}^{(2)}$ agrees with the $\mathrm{SO}(8)$ two-instanton Hilbert series in [42]. More results on $P_{2, D_{4}}^{(n)}(v)$ with $n>1$ can be found in appendix F. 


\section{$4.3 G=F_{4}$}

The divisors and curves of the non-compact $\mathfrak{n}=5$ geometry are explained in [53]. There are five compact divisors, all of which are Hirzebruch surfaces $\mathbb{F}_{n_{i}}$ of various degrees $n_{i}$. We denote them by $\mathfrak{D}_{I}(I=0,1, \ldots, 5)$. They intersect with each other like the affine dynkin diagram of $\mathfrak{f}_{4}$

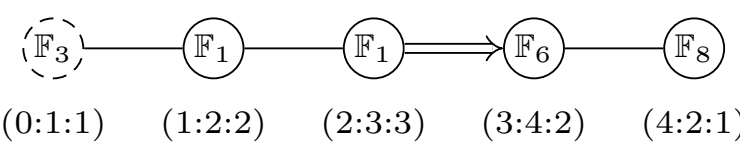

where each node corresponds to a Hirzebruch surface, and two nodes are connected if the corresponding Hirzebruch surfaces intersect at a $\mathbb{P}^{1}$ normal to their respective $\mathbb{P}^{1}$ fibers. In the diagram above we also give the ordering of the nodes $I$ and the associated marks/comarks $a_{I} / a_{I}^{\vee}$ with the notation $\left(I: a_{I}: a_{I}^{\vee}\right)$ following [61]. The $\mathbb{F}_{3}$ denoted by a dashed circle corresponds to the affine node and it intersects with the base at the $\mathbb{P}^{1}$ with normal bundle $\mathcal{O}(-5) \oplus \mathcal{O}(3) \rightarrow \mathbb{P}^{1}$. The arrow with double line means the $\mathbb{F}_{1}$ and $\mathbb{F}_{6}$ intersect at a $\mathbb{P}^{1}$ which is the double cover of the $(+1)$ curve in $\mathbb{F}_{1}$. See the illustration in figure 1. There are six linearly independent curves, which we choose for the moment to be the $\mathbb{P}^{1}$ fibers $\Sigma_{I}$ of the divisors $\mathfrak{D}_{I}$ and the $(-5)$ curve in the base denoted by $\Sigma_{B}$. Denoting their complexified Kähler moduli by $t_{I}$ and $t_{B}$, the linear combination

$$
\sum_{I=0}^{5} a_{I} t_{I}=\tau,
$$

with $a_{I}$ the marks of $\mathfrak{f}_{4}$, is the volume of the elliptic fiber. Since we will be interested in the extraction of BPS invariants from the partition function, we would like to also identify among the compact curves the Mori cone generators. They include the $\mathbb{P}^{1}$ fibers $\Sigma_{I}(I=0,1, \ldots, 4)$, as well as the $\mathbb{P}^{1}$ base of the $\mathbb{F}_{1}$ surface that intersects with $\mathbb{F}_{6}$ (see the Dynkin diagram above and the figure 7 in [53], which we reproduce in figure 1). We denote the latter curve by $\Sigma_{b}$, and it is related to $\Sigma_{B}$ by

$$
\left[\Sigma_{B}\right]=\left[\Sigma_{b}\right]+3\left[\Sigma_{0}\right]+\left[\Sigma_{1}\right] .
$$

This implies the relation between their Kähler moduli

$$
t_{B}=t_{b}+3 t_{0}+t_{1}
$$

The $C$-matrix of intersection between $\Sigma_{I}, \Sigma_{b}$ and $D_{I}{ }^{32}$

$$
C=\left(\begin{array}{ccccc}
-2 & 1 & 0 & 0 & 0 \\
1 & -2 & 1 & 0 & 0 \\
0 & 1 & -2 & 2 & 0 \\
0 & 0 & 1 & -2 & 1 \\
0 & 0 & 0 & 1 & -2 \\
0 & -1 & -1 & 0 & 0
\end{array}\right)
$$

\footnotetext{
${ }^{32}$ Note that here we use $\Sigma_{b}$ instead of $\Sigma_{B}$, which is why the matrix $C$ does not follow exactly the pattern (2.2).
} 


\begin{tabular}{|ccc|}
\hline & $r$ & fundamental weights \\
\hline & $(0,0,0,0,0,1)$ & \\
& $(0,0,0,0,0,3)$ & \\
unity & $(0,0,0,0,0,5)$ & $\omega_{i}(i=1, \ldots, 4)$ \\
& $(0,0,0,0,0,7)$ & \\
& $(0,0,0,0,0,9)$ & \\
\hline
\end{tabular}

Table 8. The $r$-fields of the $\mathfrak{n}=5$ model and the fundamental weights of $\mathfrak{f}_{4}$ which induce the same embedding $\phi: Q^{\vee} \hookrightarrow P$. All the $r$-fields and all the fundamental weights induce the same embedding as $Q^{\vee}=P$ for $\mathfrak{f}_{4}$.

The semiclassical components of the partition function can be computed using the prescription in section 2.2 with the normalisation scheme in appendix B. We obtain

$$
\begin{aligned}
-F_{(0,0)}^{\mathrm{cls}}= & \left(\frac{t_{0}}{10}+\frac{t_{1}}{5}+\frac{3 t_{2}}{10}+\frac{2 t_{3}}{5}+\frac{t_{4}}{5}\right) t_{b}^{2}+\left(\frac{3 t_{0}^{2}}{10}+\frac{t_{0} t_{1}}{5}+\frac{t_{1}^{2}}{5}+\frac{3 t_{2}^{2}}{10}+\frac{6 t_{3}^{2}}{5}+\frac{4 t_{4}^{2}}{5}+\frac{4 t_{2} t_{3}}{5}\right. \\
& \left.+\frac{2 t_{2} t_{4}}{5}+\frac{6 t_{3} t_{4}}{5}\right) t_{b}+\frac{3 t_{0}^{3}}{10}+\frac{3 t_{0}^{2} t_{1}}{10}+\frac{t_{0} t_{1}^{2}}{10}+\frac{t_{1}^{3}}{15}+\frac{t_{2}^{3}}{10}+\frac{6 t_{3}^{3}}{5}+\frac{16 t_{4}^{3}}{15}+\frac{6 t_{2} t_{3}^{2}}{5} \\
& +\frac{4 t_{2} t_{4}^{2}}{5}+\frac{12 t_{3} t_{4}^{2}}{5}+\frac{2 t_{2}^{2} t_{3}}{5}+\frac{t_{2}^{2} t_{4}}{5}+\frac{9 t_{3}^{2} t_{4}}{5}+\frac{6 t_{2} t_{3} t_{4}}{5},
\end{aligned}
$$

which is consist with the universal formula (2.18). Furthermore, using the relations (2.4), $(4.44)$ and $(4.42), F_{(0,0)}^{\text {cls }}$ can be more succinctly written as

$$
-F^{(0,0)}=\frac{1}{10} t_{\text {ell }}^{2} \tau+\frac{1}{2} t_{\text {ell }}(m, m)-\frac{3}{4} \tau(m, m)+\ldots
$$

up to $\tau^{3}$ and terms cubic in $m_{i}$, which agrees with the universal formula (2.19). Therefore the analysis in section 2.3 goes through. Here for $F_{4}$,

$$
m=\sum_{i=1}^{4} m_{i} \omega_{i}^{\vee},
$$

and

$(m, m)=m_{1}^{2}+3 m_{1} m_{2}+3 m_{2}^{2}+4 m_{1} m_{3}+8 m_{2} m_{3}+6 m_{3}^{2}+2 m_{1} m_{4}+4 m_{2} m_{4}+6 m_{3} m_{4}+2 m_{4}^{2}$.

We also find

$$
F_{(1,0)}^{\mathrm{cls}}=\frac{18}{5} t_{0}+\frac{16}{5} t_{1}+6 t_{2}+12 t_{3}+8 t_{4}+\frac{36}{5} t_{b} .
$$

Imposing the admissibility condition (2.22) and the BPS checkerboard pattern condition (2.25), which specialises to

$$
r \equiv(0,0,0,0,0,1) \quad \bmod 2,
$$

there are only five inequivalent $r$-fields, and we list their representatives in table 8 . According to the discussion in section 3 , we should classify them according to the embedding 
$\phi_{\lambda}: Q^{\vee} \hookrightarrow P$ induced by the reduced $r$-vector $\lambda$ defined in (2.28). In the case of the $\mathfrak{n}=5$ model, all the $r$-fields have the same reduced $\lambda=(0,0,0,0)$, which induces the unique embedding $\phi_{(0,0,0,0)}: Q^{\vee} \hookrightarrow P=Q^{\vee}$. As a consequence, this model has no blowup equation of the vanishing type. We notice that all the fundamental weights $\omega_{i}$ also induce the same embedding (which is not the case in all the other models.)

We use (3.33) and (3.36) to compute the one-string and two-string elliptic genera. The one-string elliptic genus does not depend on $\mathrm{SU}(2)_{x}$. Its expansion in $Q_{\tau}$ reads

$$
\mathbb{E}_{h_{F_{4}}^{(1)}}\left(v, Q_{\tau}, m_{i}\right)=v^{8} Q_{\tau}^{-4 / 3} \sum_{n=0}^{\infty} g_{1, F_{4}}^{(n)}\left(v, Q_{m_{i}}\right) Q_{\tau}^{n},
$$

where $g_{1, F_{4}}^{(n)}\left(v, Q_{m_{i}}\right)$ are rational functions. Turning off all flavor fugacities,

$$
g_{1, F_{4}}^{(n)}\left(v, Q_{m_{i}}=1\right)=\frac{1}{\left(1-v^{2}\right)^{16}} \times P_{1, F_{4}}^{(n)}(v)
$$

where

$$
\begin{aligned}
& P_{1, F_{4}}^{(0)}(v)=1+36 v^{2}+341 v^{4}+1208 v^{6}+1820 v^{8}+1208 v^{10}+341 v^{12}+36 v^{14}+v^{16}, \\
& P_{1, F_{4}}^{(1)}(v)=\left(1+v^{2}\right)^{2}\left(53+1478 v^{2}+9419 v^{4}+18036 v^{6}+9419 v^{8}+1478 v^{10}+53 v^{12}\right), \\
& P_{1, F_{4}}^{(2)}(v)=1484+36252 v^{2}+241608 v^{4}+663716 v^{6}+909400 v^{8}+\cdots+1484 v^{16} .
\end{aligned}
$$

The ellipsis in $P_{1, F_{4}}^{(2)}(v)$ is completed by making the expression palindromic. Here the leading order expression $g_{1, F_{4}}^{(0)}$ agrees with the Hilbert series of the reduced moduli space of one $F_{4}$-instanton in [41], which is not surprising since the one-string formula (3.33) reduces to the one-instanton partition function (3.35) in the $Q_{\tau} \rightarrow 0$ limit. Furthermore, higher order expressions agree with [37].

The $Q_{\tau}$ expansion of the two-string elliptic genus reads

$$
\mathbb{E}_{h_{F_{4}}^{(2)}}\left(v, x, Q_{\tau}, m_{i}\right)=v^{17} Q_{\tau}^{-17 / 6} \sum_{n=0}^{\infty} g_{2, F_{4}}^{(n)}\left(v, x, Q_{m_{i}}\right) Q_{\tau}^{n},
$$

where $g_{2, F_{4}}^{(n)}\left(v, x, Q_{m_{i}}\right)$ are rational functions. Turning of flavor fugacities and $\mathrm{SU}(2)_{x}$, we find

$$
g_{2, F_{4}}^{(n)}\left(v, x=1, Q_{m_{i}}=1\right)=\frac{1}{(1-v)^{34}(1+v)^{22}\left(1+v+v^{2}\right)^{17}} \times P_{2, F_{4}}^{(n)}(v),
$$

where

$$
\begin{aligned}
P_{2, F_{4}}^{(0)} & (v)=1+5 v+48 v^{2}+287 v^{3}+1560 v^{4}+7503 v^{5}+32316 v^{6}+125355 v^{7}+444325 v^{8} \\
& +1443572 v^{9}+4322993 v^{10}+11989241 v^{11}+30913094 v^{12}+74321701 v^{13}+167106519 v^{14} \\
& +352245510 v^{15}+697557618 v^{16}+1300152932 v^{17}+2284606168 v^{18}+3790004228 v^{19} \\
& +5943020899 v^{20}+8818128233 v^{21}+12392104012 v^{22}+16505926853 v^{23} \\
& +20851379873 v^{24}+24994963144 v^{25}+28442119825 v^{26}+30731161887 v^{27} \\
& +31533797982 v^{28}+30731161887 v^{29}+\cdots+v^{56} .
\end{aligned}
$$




$$
\begin{aligned}
P_{2, F_{4}}^{(1)} & (v)=\left(1+v^{2}\right)\left(56+386 v+3217 v^{2}+20295 v^{3}+110327 v^{4}+529286 v^{5}+2266151 v^{6}\right. \\
& +8718327 v^{7}+30479449 v^{8}+97433532 v^{9}+286304088 v^{10}+777049966 v^{11} \\
& +1956035588 v^{12}+4581942186 v^{13}+10017235514 v^{14}+20492637094 v^{15} \\
& +39315499928 v^{16}+70871529676 v^{17}+120240591034 v^{18}+192278945658 v^{19} \\
& +290168035137 v^{20}+413676858801 v^{21}+557641624668 v^{22}+711294838217 v^{23} \\
& +859008747683 v^{24}+982638991174 v^{25}+1065069893896 v^{26}+1094033908456 v^{27} \\
& \left.+1065069893896 v^{28}+\cdots+v^{56}\right) .
\end{aligned}
$$

and the ellipses are again completed by making the expressions palindromic. Here the leading order expression $g_{2, F_{4}}^{(0)}$ agrees with the Hilbert series of the reduced moduli space of two $F_{4}$-instanton in [42]. Some polynomials $P_{2, F_{4}}^{(n)}(v)$ of higher order $n$ can be found in the appendix F.

We also use the expressions of $\mathbb{E}_{1}, \mathbb{E}_{2}$ to extract the BPS invariants $N_{j_{l}, j_{r}}^{\beta}$. For this purpose, we need to use instead the Kähler moduli $t_{b}, t_{I}(I=0,1, \ldots, 4)$ associated to the Mori cone generators. The results are tabulated in appendix G. They display the proper checkerboard pattern, and reproduce the known genus 0 Gopakumar-Vafa invariants [24]. At base degree one, we also notice a pattern that the only non-vanishing BPS invariants for the curve classes $\beta=(0, k, 0,0,0,1),(0,0, k, 0,0,1)$ are

$$
N_{0, k}^{(0, k, 0,0,0,1)}=N_{0, k}^{(0,0, k, 0,0,1)}=1, \quad k=0,1, \ldots,
$$

which in fact can be proved from the one-string formula (3.33) with the help of (D.7), (D.8) and (D.9).

\section{$4.4 G=E_{6}$}

The divisors and curves of the non-compact $\mathfrak{n}=6$ geometry is explained in [53]. There are seven compact divisors, which are Hirzebruch surfaces $\mathbb{F}_{n_{i}}$ of various degrees $n_{i}$. We denote them by $D_{I}(I=0,1, \ldots, 6)$. They intersect with each other like the affine dynkin diagram of $\mathfrak{e}_{6}$

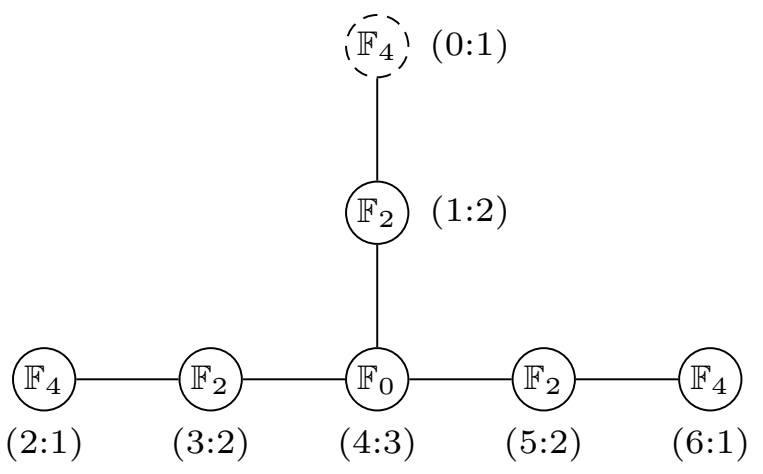

where each node corresponds to a Hirzebruch surface and two nodes are connected if the corresponding Hirzebruch surfaces intersect (see figure 5 in [53]). In the diagram above we also give the ordering of the nodes $I$ and the associated marks $a_{I}$ with the notation $\left(I: a_{I}\right)$ following [61]. The $\mathbb{F}_{4}$ denoted by a dashed circle corresponds to the affine node and it 
intersects with the base at the $\mathbb{P}^{1}$ with normal bundle $\mathcal{O}(-6) \oplus \mathcal{O}(4) \rightarrow \mathbb{P}^{1}$. There are eight linearly independent curves, which we choose for the moment to be the $\mathbb{P}^{1}$ fibers $\Sigma_{I}$ of the divisors $D_{I}$ and the $(-6)$ curve in the base denoted by $\Sigma_{B}$. Denoting their complexified Kähler moduli by $t_{I}$ and $t_{B}$, the linear combination

$$
\sum_{I=0}^{6} a_{I} t_{I}=\tau
$$

with $a_{I}$ the marks of $\mathfrak{e}_{6}$, is the volume of the elliptic fiber. We also identify the Mori cone generators. They include the $\mathbb{P}^{1}$ fibers $\Sigma_{I}(I=0,1, \ldots, 6)$, as well as the $\mathbb{P}^{1}$ base of the $\mathbb{F}_{0}$ surface in the center. We denote the last curve by $\Sigma_{b}$, and it is related to $\Sigma_{B}$ by

$$
\left[\Sigma_{B}\right]=\left[\Sigma_{b}\right]+4\left[\Sigma_{0}\right]+2\left[\Sigma_{1}\right] .
$$

This implies the following relation of their Kähler moduli

$$
t_{B}=t_{b}+4 t_{0}+2 t_{1} .
$$

The $C$-matrix of intersection between $\Sigma_{I}, \Sigma_{b}$ and $D_{I}$ is

$$
C=\left(\begin{array}{ccccccc}
-2 & 1 & 0 & 0 & 0 & 0 & 0 \\
1 & -2 & 0 & 0 & 1 & 0 & 0 \\
0 & 0 & -2 & 1 & 0 & 0 & 0 \\
0 & 0 & 1 & -2 & 1 & 0 & 0 \\
0 & 1 & 0 & 1 & -2 & 1 & 0 \\
0 & 0 & 0 & 0 & 1 & -2 & 1 \\
0 & 0 & 0 & 0 & 0 & 1 & -2 \\
0 & 0 & 0 & 0 & -2 & 0 & 0
\end{array}\right) .
$$

The semiclassical components of the partition function can be computed using the prescription in section 2.2 with the normalisation scheme in appendix B. We obtain

$$
\begin{aligned}
-F_{(0,0)}^{\mathrm{cls}}= & \left(\frac{t_{0}}{12}+\frac{t_{1}}{6}+\frac{t_{2}}{12}+\frac{t_{3}}{6}+\frac{t_{4}}{4}+\frac{t_{5}}{6}+\frac{t_{6}}{12}\right) t_{b}^{2} \\
& \left(\frac{t_{0}^{2}}{3}+\frac{t_{1}^{2}}{3}+\frac{t_{2}^{2}}{3}+\frac{t_{3}^{2}}{3}+\frac{t_{5}^{2}}{3}+\frac{t_{6}^{2}}{3}+\frac{t_{0} t_{1}}{3}+\frac{t_{2} t_{3}}{3}+\frac{t_{5} t_{6}}{3}\right) t_{b} \\
& +\frac{4 t_{0}^{3}}{9}+\frac{2 t_{1}^{3}}{9}+\frac{4 t_{2}^{3}}{9}+\frac{2 t_{3}^{3}}{9}+\frac{2 t_{5}^{3}}{9}+\frac{4 t_{6}^{3}}{9}+\frac{2 t_{0}^{2} t_{1}}{3}+\frac{t_{0} t_{1}^{2}}{3}+\frac{t_{2} t_{3}^{2}}{3}+\frac{2 t_{2}^{2} t_{3}}{3}+\frac{2 t_{5} t_{6}^{2}}{3}+\frac{t_{5}^{2} t_{6}}{3},
\end{aligned}
$$

which is consistent with the universal formula (2.19). Using the relations (2.4), (4.62), (4.60), we can express $F^{(0,0)}(t)$ in terms of the Kähler moduli $t_{\mathrm{ell}}, \tau, m_{i}(i=1, \ldots, 6)$ and find

$$
-F^{(0,0)}=\frac{1}{12} t_{\mathrm{ell}}^{2} \tau+\frac{1}{2} t_{\mathrm{ell}}(m, m)-\tau(m, m)+\ldots
$$

up to $\tau^{3}$ and terms cubic in $m_{i}$, where

$$
m=\sum_{i=1}^{6} m_{i} \omega_{i}^{\vee} .
$$




\begin{tabular}{|cccc|}
\hline & \multicolumn{2}{c}{$r$} & fundamental weights \\
\hline \multirow{4}{*}{ unity } & $(0,0,0,0,0,0,0,0)$ & $(0,0,0,0,0,0,0,2)$ & \\
& $(0,0,0,0,0,0,0,4)$ & $(0,0,0,0,0,0,0,6)$ & $\omega_{1}, \omega_{4}$ \\
& $(0,0,0,0,0,0,0,8)$ & $(0,0,0,0,0,0,0,10)$ & \\
\hline \multirow{3}{*}{ vanishing } & $(-2,0,2,0,0,0,0,0)$ & $(-2,0,2,0,0,0,0,2)$ & \\
& $(-2,0,2,0,0,0,0,4)$ & $(-2,0,2,0,0,0,0,6)$ & $\omega_{2}, \omega_{5}$ \\
& $(-2,0,2,0,0,0,0,8)$ & $(-2,0,2,0,0,0,0,10)$ & \\
\hline \multirow{2}{*}{ vanishing } & $(-2,0,0,0,0,0,2,0)$ & $(-2,0,0,0,0,0,2,2)$ & \\
& $(-2,0,0,0,0,0,2,4)$ & $(-2,0,0,0,0,0,2,6)$ & $\omega_{3}, \omega_{6}$ \\
& $(-2,0,0,0,0,0,2,8)$ & $(-2,0,0,0,0,0,2,10)$ & \\
\hline
\end{tabular}

Table 9. The $r$-fields of the $\mathfrak{n}=6$ model and the fundamental weights of $\mathfrak{e}_{6}$ which induce the same embedding $\phi: Q^{\vee} \hookrightarrow P$. They can be divided into three groups; inside each group $r$-fields or fundamental weights induce the same embedding.

It is in agreement with the universal expression (2.19), and thus the analysis in section 2.3 goes through. We also find

$$
F_{(1,0)}^{\mathrm{cls}}=\frac{9}{2} t_{0}+5 t_{1}+\frac{9}{2} t_{2}+5 t_{3}+\frac{3}{2} t_{4}+5 t_{5}+\frac{9}{2} t_{6}+8 t_{b}
$$

Imposing the admissibility condition (2.22) and the BPS checkerboard pattern condition (2.25), which specialises to

$$
r \equiv(0,0,0,0,0,0,0,0) \bmod 2,
$$

there are in total 18 inequivalent $r$-fields, and we list their representatives in table 9 . We classify them according to the embeddings $\phi_{\lambda}: Q^{\vee} \hookrightarrow P$ induced by the reduced $r$-field $\lambda$ defined in (2.28), (2.33). We also list in the table the fundamental weights which induce the same embedding.

We use (3.33) and (3.36) to compute the one-string and two-string elliptic genera. The results are again presented in terms of the reduced elliptic genera defined in (4.1).

The reduced one-string elliptic genus does not depend on $\mathrm{SU}(2)_{x}$. The expansion in $Q_{\tau}$ reads

$$
\mathbb{E}_{h_{E_{6}}^{(1)}}\left(v, Q_{\tau}, m_{i}\right)=v^{11} Q_{\tau}^{-11 / 6} \sum_{n=0}^{\infty} g_{1, E_{6}}^{(n)}\left(v, Q_{m_{i}}\right) Q_{\tau}^{n},
$$

where $g_{1, E_{6}}^{(n)}\left(v, Q_{m_{i}}\right)$ are rational functions. Turning off all flavor fugacities

$$
g_{1, E_{6}}^{(n)}\left(v, Q_{m_{i}}=1\right)=\frac{1}{\left(1-v^{2}\right)^{22}} \times P_{1, E_{6}}^{(n)}(v)
$$


where the first few orders are

$$
\begin{aligned}
& P_{1, E_{6}}^{(0)}(v)=1+56 v^{2}+945 v^{4}+6776 v^{6}+23815 v^{8}+43989 v^{10}+\cdots+v^{22}, \\
& P_{1, E_{6}}^{(1)}(v)=79+3774 v^{2}+54206 v^{4}+337457 v^{6}+1067286 v^{8}+1862806 v^{10}+\cdots+79 v^{22}, \\
& P_{1, E_{6}}^{(2)}(v)=3239+130034 v^{2}+1603334 v^{4}+8798601 v^{6}+25393522 v^{8}+42223058 v^{10}+\cdots+3239 v^{22} .
\end{aligned}
$$

The ellipses are completed by making the expression palindromic. Here $g_{1, E_{6}}^{(0)}$ agrees with the Hilbert series of reduced one $E_{6}$-instanton moduli space [41], while higher order contributions agree with [37].

The $Q_{\tau}$ expansion of the two-string elliptic genus reads

$$
\mathbb{E}_{h_{E_{6}}^{(2)}}\left(v, x, Q_{\tau}, m_{i}\right)=v^{23} Q_{\tau}^{-23 / 6} \sum_{n=0}^{\infty} g_{2, E_{6}}^{(n)}\left(v, x, Q_{m_{i}}\right) Q_{\tau}^{n} .
$$

Turning off flavor fugacities and $\mathrm{SU}(2)_{x}$, we obtain

$$
g_{2, E_{6}}^{(n)}\left(v, x, Q_{m_{i}}=1\right)=\frac{1}{(1-v)^{46}(1+v)^{32}\left(1+v+v^{2}\right)^{23}} \times P_{2, E_{6}}^{(n)}(v),
$$

where the first few orders are

$$
\begin{aligned}
& P_{2, E_{6}}^{(0)}(v)=1+9 v+94 v^{2}+739 v^{3}+5121 v^{4}+31432 v^{5}+173895 v^{6}+874485 v^{7}+4036298 v^{8} \\
& +17200367 v^{9}+68039474 v^{10}+250943933 v^{11}+866242068 v^{12}+2807705547 v^{13} \\
& \quad+8569454706 v^{14}+24690503239 v^{15}+67304396959 v^{16}+173919980352 v^{17} \\
& +426790882149 v^{18}+996158535441 v^{19}+2214670938701 v^{20}+4695878015170 v^{21} \\
& +9507297417908 v^{22}+18398716114730 v^{23}+34066083855696 v^{24}+60399840583490 v^{25} \\
& +102628223553496 v^{26}+167232472484542 v^{27}+261500117384417 v^{28}+392614934492341 v^{29} \\
& +566271723784347 v^{30}+784947220008032 v^{31}+1046126546231772 v^{32}+1340924322289616 v^{33} \\
& +1653587141756229 v^{34}+1962268356880815 v^{35}+2241216639463322 v^{36}+2464163123099051 v^{37} \\
& +2608327634962043 v^{38}+2658213934310966 v^{39}+\cdots+v^{78} .
\end{aligned}
$$

Note that $g_{2, E_{6}}^{(0)}$ agrees with the Hilbert series of reduced two $E_{6}$-instanton moduli space [42].

We also use the expressions of $\mathbb{E}_{1}, \mathbb{E}_{2}$ to extract the BPS invariants $N_{j_{l}, j_{r}}^{\beta}$. For this purpose, we need to use the Kähler moduli $t_{I}, t_{b}(I=0,1, \ldots, 6)$ associated to the Mori cone generators. The results are tabulated in appendix G. They display the proper checkerboard pattern, and reproduce the known genus 0 Gopakumar-Vafa invariants [24]. At base degree one, we notice the interesting pattern that the only non-vanishing BPS invariants for the curve classes $\beta=(0, k, 0,0,0,0,0,1),(0,0,0, k, 0,0,0,1),(0,0,0,0,0, k, 0,1)$, $(0,0,0,0, k, 0,0,1)$ are

$$
N_{0, k-1 / 2}^{(0, k, 0,0,0,0,0,1)}=N_{0, k-1 / 2}^{(0,0,0, k, 0,0,0,1)}=N_{0, k-1 / 2}^{(0,0,0,0,0, k, 0,1)}=N_{0, k+1 / 2}^{(0,0,0,0, k, 0,0,1)}=1, \quad k=1,2, \ldots,
$$

which in fact can be proved from the one-string formula (3.33) with the help of (D.7), (D.8) and (D.9). 


\section{$4.5 G=E_{7}$}

The divisors and curves of the non-compact $\mathfrak{n}=8$ geometry is explained in [53]. There are eight compact divisors, which are Hirzebruch surfaces $\mathbb{F}_{n_{i}}$ of various degrees $n_{i}$. We denote them by $D_{I}(I=0,1, \ldots, 7)$. They intersect with each other like the affine dynkin diagram of $\mathfrak{e}_{7}$

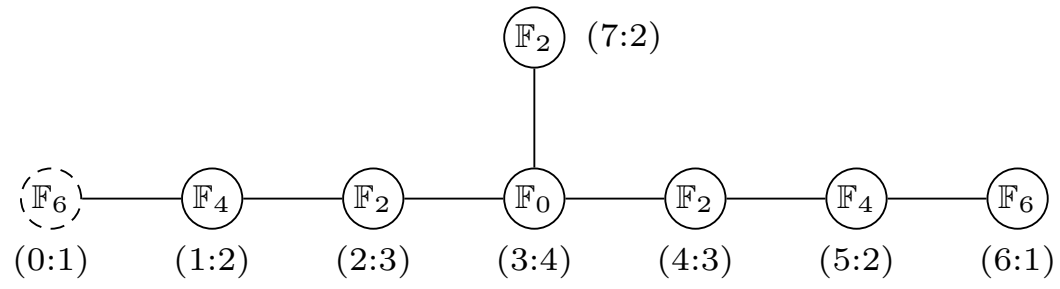

In the diagram above we also give the ordering of the nodes $I$ and the associated marks $a_{I}$ with the notation $\left(I: a_{I}\right)$. The $\mathbb{F}_{6}$ denoted by a dashed circle corresponds to the affine node and it intersects with the base at the $\mathbb{P}^{1}$ with normal bundle $\mathcal{O}(-8) \oplus \mathcal{O}(6) \rightarrow \mathbb{P}^{1}$. There are nine linearly independent curves, which we choose for the moment to be the $\mathbb{P}^{1}$ fibers $\Sigma_{I}$ of the divisors $D_{I}$ and the $(-8)$ curve in the base denoted by $\Sigma_{B}$. Denoting their complexified Kähler moduli by $t_{I}$ and $t_{B}$, the linear combination

$$
\sum_{I=0}^{7} a_{I} t_{I}=\tau
$$

with $a_{I}$ the marks of $\mathfrak{e}_{7}$, is the volume of the elliptic fiber. We identify the Mori cone generators. They include the $\mathbb{P}^{1}$ fibers $\Sigma_{I}(I=0,1, \ldots, 7)$, as well as the $\mathbb{P}^{1}$ base of the $\mathbb{F}_{0}$ surface in the middle. We denote the last curve by $\Sigma_{b}$, which is related to $\Sigma_{B}$ by

$$
\left[\Sigma_{B}\right]=\left[\Sigma_{b}\right]+6\left[\Sigma_{0}\right]+4\left[\Sigma_{1}\right]+2\left[\Sigma_{2}\right] .
$$

Their Kähler moduli are consequently related by

$$
t_{B}=t_{b}+6 t_{0}+4 t_{1}+2 t_{2} .
$$

The $C$-matrix of intersection between $\Sigma_{I}, \Sigma_{b}$ and $D_{I}$ is

$$
C=\left(\begin{array}{cccccccc}
-2 & 1 & 0 & 0 & 0 & 0 & 0 & 0 \\
1 & -2 & 1 & 0 & 0 & 0 & 0 & 0 \\
0 & 1 & -2 & 1 & 0 & 0 & 0 & 0 \\
0 & 0 & 1 & -2 & 1 & 0 & 0 & 1 \\
0 & 0 & 0 & 1 & -2 & 1 & 0 & 0 \\
0 & 0 & 0 & 0 & 1 & -2 & 1 & 0 \\
0 & 0 & 0 & 0 & 0 & 1 & -2 & 0 \\
0 & 0 & 0 & 1 & 0 & 0 & 0 & -2 \\
0 & 0 & 0 & -2 & 0 & 0 & 0 & 0
\end{array}\right)
$$


The semiclassical components of the partition function can be computed using the prescription in section 2.2 with the normalisation scheme in appendix B. We obtain

$$
\begin{aligned}
-F_{(0,0)}^{\mathrm{cls}}= & \left(\frac{t_{0}}{16}+\frac{t_{1}}{8}+\frac{3 t_{2}}{16}+\frac{t_{3}}{4}+\frac{3 t_{4}}{16}+\frac{t_{5}}{8}+\frac{t_{6}}{16}+\frac{t_{7}}{8}\right) t_{b}^{2}+\left(\frac{3 t_{0}^{2}}{8}+\frac{t_{1}^{2}}{2}+\frac{3 t_{2}^{2}}{8}+\frac{3 t_{4}^{2}}{8}+\frac{t_{5}^{2}}{2}+\frac{3 t_{6}^{2}}{8}\right. \\
& \left.+\frac{t_{7}^{2}}{4}+\frac{t_{0} t_{1}}{2}+\frac{t_{0} t_{2}}{4}+\frac{t_{1} t_{2}}{2}+\frac{t_{4} t_{5}}{2}+\frac{t_{4} t_{6}}{4}+\frac{t_{5} t_{6}}{2}\right) t_{b}+\frac{3 t_{0}^{3}}{4}+\frac{2 t_{1}^{3}}{3}+\frac{t_{2}^{3}}{4}+\frac{t_{4}^{3}}{4}+\frac{2 t_{5}^{3}}{3} \\
& +\frac{3 t_{6}^{3}}{4}+\frac{t_{7}^{3}}{6}+\frac{3 t_{0}^{2} t_{1}}{2}+\frac{3 t_{0}^{2} t_{2}}{4}+t_{0} t_{1}^{2}+\frac{t_{0} t_{2}^{2}}{4}+t_{0} t_{1} t_{2}+\frac{t_{1} t_{2}^{2}}{2}+t_{4} t_{5}^{2}+\frac{3 t_{4} t_{6}^{2}}{4}+\frac{3 t_{5} t_{6}^{2}}{2} \\
& +t_{1}^{2} t_{2}+\frac{t_{4}^{2} t_{5}}{2}+\frac{t_{4}^{2} t_{6}}{4}+t_{5}^{2} t_{6}+t_{4} t_{5} t_{6},
\end{aligned}
$$

which is consistent with the universal formula (2.19). Using the relations (2.4), (4.78) and (4.76), we can express $F^{(0,0)}(t)$ in terms of the Kähler moduli $t_{\mathrm{ell}}, \tau, m_{i}(i=1, \ldots, 7)$ and find

$$
-F^{(0,0)}=\frac{1}{16} t_{\text {ell }}^{2} \tau+\frac{1}{2} t_{\text {ell }}(m, m)-\frac{3}{2} \tau(m, m)+\ldots
$$

up to $\tau^{3}$ and terms cubic in $m_{i}$, where

$$
m=\sum_{i=1}^{7} m_{i} \omega_{i}^{\vee}
$$

It is in agreement with the universal expression (2.19), and therefore the analysis in section 2.3 goes through, which then leads to the elliptic blowup equations (3.4). We also find

$$
F_{(1,0)}^{\mathrm{cls}}=\frac{51}{8} t_{0}+\frac{35}{4} t_{1}+\frac{57}{8} t_{2}+\frac{3}{2} t_{3}+\frac{57}{8} t_{4}+\frac{35}{4} t_{5}+\frac{51}{8} t_{6}+\frac{11}{4} t_{b} .
$$

Imposing the admissibility condition (2.22) and the BPS checkerboard pattern condition (2.25), which specialises to

$$
r \equiv(0,0,0,0,0,0,0,0,0) \bmod 2
$$

there are in total 16 inequivalent $r$-fields, and we list their representatives in table 10 . We classify them according to the embeddings $\phi_{\lambda}: Q^{\vee} \hookrightarrow P$ induced by the reduced $r$-field $\lambda$ defined in (2.28), (2.33). We also list in the table the fundamental weights which induce the same embedding.

We use (3.33) and (3.36) to compute the one-string and two-string elliptic genera and convert them to reduced versions. The one string elliptic genus when expanded in $Q_{\tau}$ reads

$$
\mathbb{E}_{h_{E_{7}}^{(1)}}\left(v, Q_{\tau}, m_{i}\right)=v^{17} Q_{\tau}^{-17 / 6} \sum_{n=0}^{\infty} g_{1, E_{7}}^{(n)}\left(v, Q_{m_{i}}\right) Q_{\tau}^{n},
$$

where $g_{1, E_{7}}^{(n)}\left(v, Q_{m_{i}}\right)$ are rational functions. When all flavor fugacities are turned off

$$
g_{1, E_{7}}^{(n)}\left(v, Q_{m_{i}}=1\right)=\frac{1}{\left(1-v^{2}\right)^{34}} \times P_{n, E_{7}}^{(1)}(v),
$$




\begin{tabular}{|cccc|}
\hline & \multicolumn{2}{|c}{$r$} & fundamental weights \\
\hline \multirow{4}{*}{ unity } & $(0,0,0,0,0,0,0,0,0)$ & $(0,0,0,0,0,0,0,0,2)$ & \\
& $(0,0,0,0,0,0,0,0,4)$ & $(0,0,0,0,0,0,0,0,6)$ & $\omega_{1}, \omega_{2}, \omega_{3}, \omega_{5}$ \\
& $(0,0,0,0,0,0,0,0,8)$ & $(0,0,0,0,0,0,0,0,10)$ & \\
& $(0,0,0,0,0,0,0,0,12)$ & $(0,0,0,0,0,0,0,0,14)$ & \\
\hline \multirow{3}{*}{ vanishing } & $(-2,0,0,0,0,0,2,0,0)$ & $(-2,0,0,0,0,0,2,0,2)$ & \\
& $(-2,0,0,0,0,0,2,0,4)$ & $(-2,0,0,0,0,0,2,0,6)$ & $\omega_{4}, \omega_{6}, \omega_{7}$ \\
& $(-2,0,0,0,0,0,2,0,8)$ & $(-2,0,0,0,0,0,2,0,10)$ & \\
& $(-2,0,0,0,0,0,2,0,12)$ & $(-2,0,0,0,0,0,2,0,14)$ & \\
\hline
\end{tabular}

Table 10. The $r$-fields of the $\mathfrak{n}=8$ model and the fundamental weights of $\mathfrak{e}_{7}$ which induce the same embedding $\phi: Q^{\vee} \hookrightarrow P$. They can be divided into two groups; inside each group $r$-fields or fundamental weights induce the same embedding.

where the leading order contributions are

$$
\begin{aligned}
P_{1, E_{7}}^{(0)}(v)= & \left(1+v^{2}\right)\left(1+98 v^{2}+3312 v^{4}+53305 v^{6}+468612 v^{8}+2421286 v^{10}+7664780 v^{12}\right. \\
& \left.+15203076 v^{14}+19086400 v^{16}+15203076 v^{18}+\cdots+v^{32}\right), \\
P_{1, E_{7}}^{(1)}(v)= & \left(1+v^{2}\right)\left(134+11593 v^{2}+345521 v^{4}+4931707 v^{6}+38850151 v^{8}+182614170 v^{10}\right. \\
& \left.+536726278 v^{12}+1014596958 v^{14}+1252490096 v^{16}+1014596958 v^{18}+\cdots+v^{32}\right), \\
P_{1, E_{7}}^{(2)}(v)= & \left(1+v^{2}\right)\left(9179+693316 v^{2}+18210733 v^{4}+231525774 v^{6}+1645739978 v^{8}\right. \\
& +7093827388 v^{10}+19507715662 v^{12}+35350906224 v^{14}+43009574252 v^{16} \\
& \left.+35350906224 v^{18} \cdots+v^{32}\right) .
\end{aligned}
$$

where the ellipses are completed by palindrome. Here $g_{1, E_{7}}^{(0)}$ agrees with the Hilbert series of reduced one $E_{7}$-instanton moduli space in [41], while higher order contributions agree with [37].

The $Q_{\tau}$ expansion of the two-string elliptic genus reads

$$
\mathbb{E}_{h_{E_{7}}^{(2)}}\left(v, x, Q_{\tau}, m_{i}\right)=v^{35} Q_{\tau}^{-35 / 6} \sum_{n=0}^{\infty} g_{2, E_{7}}^{(n)}\left(v, x, Q_{m_{i}}\right) Q_{\tau}^{n} .
$$

Turning off SU $(2)_{x}$ and flavours, we have

$$
g_{2, E_{7}}^{(n)}\left(v, x, Q_{m_{i}}=1\right)=\frac{1}{(1-v)^{70}(1+v)^{52}\left(1+v+v^{2}\right)^{35}} \times P_{n, E_{7}}^{(2)}(v) .
$$

We have computed $P_{n, E_{7}}^{(2)}(v)$ for $n=0,1$ which we put in the appendix F. Indeed, our $g_{2, E_{7}}^{(0)}$ agrees with the Hibert series of reduced two $E_{7}$-instanton moduli space in [42].

We also use the expressions of $\mathbb{E}_{1}, \mathbb{E}_{2}$ to extract the BPS invariants $N_{j_{l}, j_{r}}^{\beta}$. For this purpose, we need to use the Kähler moduli $t_{I}, t_{b}(I=0,1, \ldots, 7)$ associated to the Mori cone generators. The results are tabulated in appendix G. They display the proper checkerboard 
pattern, and reproduce the known genus zero Gopakumar-Vafa invariants [24]. At base degree one, we notice the interesting pattern that the only non-vanishing BPS invariants for the curve classes $\beta=(0,0, k, 0,0,0,0,0,1),(0,0,0,0, k, 0,0,0,1),(0,0,0,0,0,0,0, k, 1)$, $(0,0,0, k, 0,0,0,0,1)$ are

$N_{0, k-1 / 2}^{(0,0, k, 0,0,0,0,0,1)}=N_{0, k-1 / 2}^{(0,0,0,0, k, 0,0,0,1)}=N_{0, k-1 / 2}^{(0,0,0,0,0,0,0, k, 1)}=N_{0, k+1 / 2}^{(0,0,0, k, 0,0,0,0,1)}=1, \quad, \quad k=1,2, \ldots$,

which in fact can be proved from the one-string formula (3.33) with the help of (D.7), (D.8) and (D.9).

\section{6 $G=E_{8}$}

The divisors and curves of the non-compact $\mathfrak{n}=12$ geometry is explained in [53]. There are nine compact divisors, which are Hirzebruch surfaces of various degrees. We denote them by $D_{I}(I=0,1, \ldots, 8)$. They intersect with each other like the affine dynkin diagram of $\mathfrak{e}_{8}$

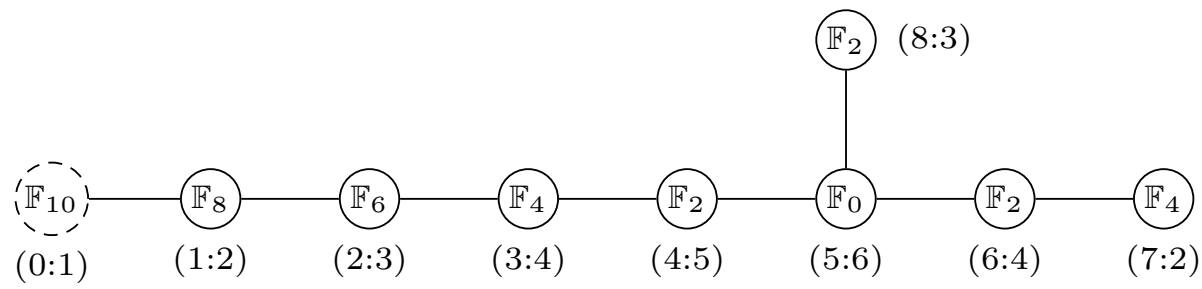

In the diagram above we also give the ordering of the nodes $I$ and the associated marks $a_{I}$ with the notation $\left(I: a_{I}\right)$. The $\mathbb{F}_{10}$ denoted by a dashed circle corresponds to the affine node and it intersects with the base at the $\mathbb{P}^{1}$ with normal bundle $\mathcal{O}(-12) \oplus \mathcal{O}(10) \rightarrow \mathbb{P}^{1}$. There are ten linearly independent curves, which we choose for the moment to be the $\mathbb{P}^{1}$ fibers $\Sigma_{I}$ of the divisors $D_{I}$ and the $(-12)$ curve in the base denoted by $\Sigma_{B}$. Let $t_{I}$ and $t_{B}$ be their complexified Kähler moduli. The linear combination

$$
\sum_{I=0}^{8} a_{I} t_{I}=\tau
$$

with $a_{I}$ marks of $\mathfrak{e}_{8}$, is the volume of the elliptic fiber. We identify the Mori cone generators. They include $\mathbb{P}^{1}$ fibers $\Sigma_{I}(I=0,1, \ldots, 8)$ as well as the $\mathbb{P}^{1}$ base of the $\mathbb{F}_{0}$ surface in the center. We denote the latter by $\Sigma_{b}$, which is related to $\Sigma_{B}$ by

$$
\left[\Sigma_{B}\right]=\left[\Sigma_{b}\right]+10\left[\Sigma_{0}\right]+8\left[\Sigma_{1}\right]+6\left[\Sigma_{2}\right]+4\left[\Sigma_{3}\right]+2\left[\Sigma_{4}\right]
$$

which implies

$$
t_{B}=t_{b}+10 t_{0}+8 t_{1}+6 t_{2}+4 t_{3}+2 t_{4} .
$$


The $C$-matrix of intersection between $\Sigma_{I}, \Sigma_{b}$ and $D_{I}$ is

$$
C=\left(\begin{array}{ccccccccc}
-2 & 1 & 0 & 0 & 0 & 0 & 0 & 0 & 0 \\
1 & -2 & 1 & 0 & 0 & 0 & 0 & 0 & 0 \\
0 & 1 & -2 & 1 & 0 & 0 & 0 & 0 & 0 \\
0 & 0 & 1 & -2 & 1 & 0 & 0 & 0 & 0 \\
0 & 0 & 0 & 1 & -2 & 1 & 0 & 0 & 0 \\
0 & 0 & 0 & 0 & 1 & -2 & 1 & 0 & 1 \\
0 & 0 & 0 & 0 & 0 & 1 & -2 & 1 & 0 \\
0 & 0 & 0 & 0 & 0 & 0 & 1 & -2 & 0 \\
0 & 0 & 0 & 0 & 0 & 1 & 0 & 0 & -2 \\
0 & 0 & 0 & 0 & 0 & -2 & 0 & 0 & 0
\end{array}\right) .
$$

The semiclassical components of the partition function can be computed using the prescription in section 2.2 with the normalisation scheme in appendix B. We obtain

$$
\begin{aligned}
-F_{(0,0)}^{\mathrm{cls}}= & \left(\frac{t_{0}}{24}+\frac{t_{1}}{12}+\frac{t_{2}}{8}+\frac{t_{3}}{6}+\frac{5 t_{4}}{24}+\frac{t_{5}}{4}+\frac{t_{6}}{6}+\frac{t_{7}}{12}+\frac{t_{8}}{8}\right) t_{b}^{2} \\
& +\left(\frac{5 t_{0}^{2}}{12}+\frac{2 t_{1}^{2}}{3}+\frac{3 t_{2}^{2}}{4}+\frac{2 t_{3}^{2}}{3}+\frac{5 t_{4}^{2}}{12}+\frac{t_{6}^{2}}{3}+\frac{t_{7}^{2}}{3}+\frac{t_{8}^{2}}{4}+\frac{2 t_{0} t_{1}}{3}+\frac{t_{0} t_{2}}{2}+\frac{t_{0} t_{3}}{3}+\frac{t_{0} t_{4}}{6}\right. \\
& \left.+t_{1} t_{2}+\frac{2 t_{1} t_{3}}{3}+t_{2} t_{3}+\frac{t_{1} t_{4}}{3}+\frac{t_{2} t_{4}}{2}+\frac{2 t_{3} t_{4}}{3}+\frac{t_{6} t_{7}}{3}\right) t_{b} \\
& +\frac{25 t_{0}^{3}}{18}+\frac{16 t_{1}^{3}}{9}+\frac{3 t_{2}^{3}}{2}+\frac{8 t_{3}^{3}}{9}+\frac{5 t_{4}^{3}}{18}+\frac{2 t_{6}^{3}}{9}+\frac{4 t_{7}^{3}}{9}+\frac{t_{8}^{3}}{6}+\frac{10 t_{0}^{2} t_{1}}{3}+\frac{5 t_{0}^{2} t_{2}}{2}+\frac{5 t_{0}^{2} t_{3}}{3}+\frac{5 t_{0}^{2} t_{4}}{6} \\
& +\frac{8 t_{0} t_{1}^{2}}{3}+\frac{3 t_{0} t_{2}^{2}}{2}+\frac{2 t_{0} t_{3}^{2}}{3}+\frac{t_{0} t_{4}^{2}}{6}+4 t_{0} t_{1} t_{2}+\frac{8 t_{0} t_{1} t_{3}}{3}+2 t_{0} t_{2} t_{3}+\frac{4}{3} t_{0} t_{1} t_{4}+t_{0} t_{2} t_{4}+\frac{2 t_{0} t_{3} t_{4}}{3} \\
& +3 t_{1} t_{2}^{2}+\frac{4 t_{1} t_{3}^{2}}{3}+2 t_{2} t_{3}^{2}+\frac{t_{1} t_{4}^{2}}{3}+\frac{t_{2} t_{4}^{2}}{2}+\frac{2 t_{3} t_{4}^{2}}{3}+\frac{2 t_{6} t_{7}^{2}}{3}+4 t_{1}^{2} t_{2}+\frac{8 t_{1}^{2} t_{3}}{3}+3 t_{2}^{2} t_{3}+4 t_{1} t_{2} t_{3} \\
& +\frac{4 t_{1}^{2} t_{4}}{3}+\frac{3 t_{2}^{2} t_{4}}{2}+\frac{4 t_{3}^{2} t_{4}}{3}+2 t_{1} t_{2} t_{4}+\frac{4 t_{1} t_{3} t_{4}}{3}+2 t_{2} t_{3} t_{4}+\frac{t_{6}^{2} t_{7}}{3},
\end{aligned}
$$

which is consistent with (2.19). Using the relations (2.4), (4.93), (4.91), we can express $F^{(0,0)}(\underline{t})$ in terms of the Kähler moduli $t_{\mathrm{ell}}, \tau, m_{i}(i=1, \ldots, 8)$ and find

$$
-F^{(0,0)}=\frac{1}{24} t_{\mathrm{ell}}^{2} \tau+\frac{1}{2} t_{\mathrm{ell}}(m, m)-\frac{5}{2} \tau(m, m)+\ldots
$$

up to $\tau^{3}$ and terms cubic in $m_{i}$, where

$$
m=\sum_{i=1}^{8} m_{i} \omega_{i}^{\vee} .
$$

It is in agreement with the universal expression (2.19), and therefore the analysis in section 2.3 goes through leading to the elliptic blowup equations (3.4). We also find

$$
F_{(1,0)}^{\mathrm{cls}}=\frac{41}{4} t_{0}+\frac{33}{2} t_{1}+\frac{75}{4} t_{2}+17 t_{3}+\frac{45}{4} t_{4}+\frac{3}{2} t_{5}+5 t_{6}+\frac{9}{2} t_{7}+\frac{11}{4} t_{8}+10 t_{b} .
$$




\begin{tabular}{|c|c|c|c|}
\hline & \multicolumn{2}{|c|}{$r$} & fundamental weights \\
\hline \multirow{6}{*}{ unity } & $(0,0,0,0,0,0,0,0,0,0)$ & $(0,0,0,0,0,0,0,0,0,2)$ & \multirow{6}{*}{$\omega_{i}(i=1, \ldots, 8)$} \\
\hline & $(0,0,0,0,0,0,0,0,0,4)$ & $(0,0,0,0,0,0,0,0,0,6)$ & \\
\hline & $(0,0,0,0,0,0,0,0,0,8)$ & $(0,0,0,0,0,0,0,0,0,10)$ & \\
\hline & $(0,0,0,0,0,0,0,0,0,12)$ & $(0,0,0,0,0,0,0,0,0,14)$ & \\
\hline & $(0,0,0,0,0,0,0,0,0,16)$ & $(0,0,0,0,0,0,0,0,0,18)$ & \\
\hline & $(0,0,0,0,0,0,0,0,0,20)$ & $(0,0,0,0,0,0,0,0,0,22)$ & \\
\hline
\end{tabular}

Table 11. The $r$-fields of the $\mathfrak{n}=12$ model and the fundamental weights of $\mathfrak{e}_{8}$ which induce the same embedding $\phi: Q^{\vee} \hookrightarrow P$. All the $r$-fields and all the fundamental weights induce the same embedding as $Q^{\vee}=P$ for $\mathfrak{e}_{8}$.

Imposing the admissibility condition (2.22) and the BPS checkerboard pattern condition (2.25), which specialises to

$$
r \equiv(0,0,0,0,0,0,0,0,0,0) \bmod 2
$$

there are in total 12 inequivalent $r$-fields, and we list their representatives in table 11. All of them have the same reduced $\lambda$ which induces the same embedding $\phi_{\lambda}: Q^{\vee} \hookrightarrow P=Q^{\vee}$. In this special case, there is no vanishing blowup equations.

We use (3.33) and (3.36) to compute the one-string and two-string elliptic genera and convert them to reduced versions. The one-string reduced elliptic genus in $Q_{\tau}$ expansion reads

$$
\mathbb{E}_{h_{E_{8}}^{(1)}}\left(v, Q_{\tau}, m_{i}\right)=v^{29} Q_{\tau}^{-29 / 6} \sum_{n=0}^{\infty} g_{1, E_{8}}^{(n)}\left(v, Q_{m_{i}}\right) Q_{\tau}^{n},
$$

where $g_{1, E_{8}}^{(n)}$ are rational functions. Turning off flavor fugacities

$$
g_{1, E_{8}}^{(n)}\left(v, Q_{m_{i}}=1\right)=\frac{1}{\left(1-v^{2}\right)^{58}} \times P_{n, E_{8}}^{(1)}(v)
$$

where the leading orders are

$$
\begin{aligned}
P_{0, E_{8}}^{(1)}(v) & =\left(1+v^{2}\right)\left(1+189 v^{2}+14080 v^{4}+562133 v^{6}+13722599 v^{8}+220731150 v^{10}\right. \\
& +2454952400 v^{12}+19517762786 v^{14}+113608689871 v^{16}+492718282457 v^{18} \\
& +1612836871168 v^{20}+4022154098447 v^{22}+7692605013883 v^{24}+11332578013712 v^{26} \\
& \left.+12891341012848 v^{28}+11332578013712 v^{30}+\cdots+v^{56}\right), \\
P_{1, E_{8}}^{(1)}(v) & =249+43435 v^{2}+2998484 v^{4}+111587988 v^{6}+2558096217 v^{8}+38985250263 v^{10} \\
& +415090167480 v^{12}+3197400818096 v^{14}+18281159666407 v^{16}+79099752469353 v^{18} \\
& +262872507223458 v^{20}+678620928038790 v^{22}+1372471431431505 v^{24} \\
& +2187800775100695 v^{26}+2759575276449180 v^{28}+2759575276449180 v^{30}+\cdots+v^{58},
\end{aligned}
$$




$$
\begin{aligned}
P_{2, E_{8}}^{(1)}(v) & =31374+4996185 v^{2}+316301853 v^{4}+10844316461 v^{6}+230109165319 v^{8} \\
& +3262175735364 v^{10}+32482207865920 v^{12}+235331998114532 v^{14} \\
& +1273365718136904 v^{16}+5249113972780491 v^{18}+16738824444898167 v^{20} \\
& +41781447040327605 v^{22}+82360817736515085 v^{24}+129037047832755990 v^{26} \\
& +161349436368883950 v^{28}+161349436368883950 v^{30}+\cdots+v^{58} .
\end{aligned}
$$

where the ellipses are completed by palindome. Note $g_{1, E_{8}}^{(0)}$ indeed agrees with the Hilbert series of reduced one $E_{8}$-instanton moduli space in [41]. Higher order contributions agree with [37].

The two-string reduced elliptic genus in $Q_{\tau}$ expansion reads

$$
\mathbb{E}_{h_{E_{8}}^{(2)}}\left(v, x, Q_{\tau}, m_{i}\right)=v^{59} Q_{\tau}^{-59 / 6} \sum_{n=0}^{\infty} g_{2, E_{8}}^{(n)}\left(v, x, Q_{m_{i}}\right) Q_{\tau}^{n},
$$

where $g_{2, E_{8}}^{(n)}\left(v, x, Q_{m_{i}}\right)$ are rational functions. Turning off flavor fugacities and $\mathrm{SU}(2)_{x}$

$$
g_{2, E_{8}}^{(n)}\left(v, x, Q_{m_{i}}=1\right)=\frac{1}{(1-v)^{118}(1+v)^{92}\left(1+v+v^{2}\right)^{59}} \times P_{2, E_{8}}^{(n)}(v) .
$$

We have computed $g_{2, E_{8}}^{(0)}$ which indeed agrees with the Hilbert series of two $E_{8}$-instanton reduced moduli space in [42].

We also use the expressions of $\mathbb{E}_{1}, \mathbb{E}_{2}$ to extract the BPS invariants $N_{j_{l}, j_{r}}^{\beta}$. For this purpose, we need to use the Kähler moduli $t_{I}, t_{b}(I=0,1, \ldots, 8)$ associated to the Mori cone generators. The results are tabulated in appendix $\mathrm{G}$. They display the proper checkerboard pattern, and reproduce the known genus zero Gopakumar-Vafa invariants [24]. At base degree one, we notice a pattern that the only non-vanishing BPS invariants for the curve classes $\beta=(0,0,0,0, k, 0,0,0,0,1),(0,0,0,0,0,0, k, 0,0,1),(0,0,0,0,0,0,0,0, k, 1)$, $(0,0,0,0,0, k, 0,0,0,1)$ are

$N_{0, k-1 / 2}^{(0,0,0,0, k, 0,0,0,0,1)}=N_{0, k-1 / 2}^{(0,0,0,0,0,0, k, 0,0,1)}=N_{0, k-1 / 2}^{(0,0,0,0,0,0,0,0, k, 1)}=N_{0, k+1 / 2}^{(0,0,0,0,0, k, 0,0,0,1)}=1, \quad k=1,2, \ldots$,

which in fact can be proved from the one-string formula (3.33) with the help of (D.7), (D.8) and (D.9).

\section{On the relation with 4 d SCFTs of type $H_{G}^{(k)}$}

The purpose of this section is to connect the $k$-string elliptic genera $\mathbb{E}_{h_{G}^{(k)}}$ for the minimal $\mathcal{N}=(1,0) 6 \mathrm{~d}$ SCFTs with $G=A_{2}, D_{4}, F_{4}, E_{6,7,8}$ discussed above to the superconformal indices of the $\mathcal{N}=24 \mathrm{~d}$ SCFTs of rank $k$ denoted by $H_{G}^{(k)}$. The simplest series of $\mathcal{N}=2$ SCFTs namely $H_{G}^{(1)}$ can be obtained by geometric engineering on non-compact del Pezzo geometries and contains the Minahan-Nemeschansky theories. The main result is an extension of a surprising conjecture by Del Zotto-Lockhart from the rank one case [37] to the higher rank cases. To be precise [37] recognised that the one-string elliptic 
genus $\mathbb{E}_{h_{G}^{(1)}}\left(Q_{\tau}, v\right)$ can be decomposed in terms of a seemingly more fundamental function $L_{G}\left(Q_{\tau}, v\right)$, which for special choices of $Q_{\tau}$ and $v$ specialises to the Hall-Littlewood index or the Schur index of the $H_{G}^{(1)}$ theories. With the two string elliptic genera computed in our previous sections, we are able to study this conjectural relation at rank two and in principle at arbitrary rank, and find indeed that similar striking relations exist.

We first review some basic properties of $4 \mathrm{~d}$ rank $k$ type $H_{G}^{(k)}-$ and $\widetilde{H}_{G}^{(k)}$ theories, including their class $\mathcal{S}$ theory construction, and then review the superconformal indices of $4 \mathrm{~d}$ SCFTs in various physically motivated limits as well as the methods to compute them. Next we state the conjectural relation at rank one from [37], and explain in some detail the new relations at rank two for all $G$. We also extend the analysis to some rank three cases. For all choices of rank and $G$ we analysed, the surprising relation between elliptic genera and superconformal indices exists. We define an intermediate function at rank $k$ called $L_{G}^{(k)}{ }^{33}$ This function is on the one hand the ingredient of $k$-string elliptic genus, on the other hand gives the Hall-Littelwood index and Schur index of $H_{G}^{(k)}$ theories at special choices of parameters. This general structure allows us to calculate the latter indices efficiently from the $\mathbb{E}_{h_{G}^{(k)}}$ that are determined from the elliptic blowup equations.

\subsection{Rank $k H_{G}$ theories}

The $4 \mathrm{~d} \mathcal{N}=2$ SCFTs $H_{G}^{(k)}$ are well known to exist for $G=\emptyset, A_{1}, A_{2}, D_{4}, E_{6,7,8}$ and $k=1,2,3 \ldots[62-66] .{ }^{34}$ In type IIB superstring theory, they are realized as the worldvolume theory for $k$ multiple D3-branes probing a stack of exotic seven-branes. Such seven-branes in F-theory are defined as codimension one singularities with Kodaire type $I I, I I I, I V, I_{0}^{*}, I V^{*}, I I I^{*}$, and $I I^{*}$, which give the gauge symmetries $G$ for the low energy 8d SYM theories. The number $k$ is usually called the rank of $H_{G}$ theories. For example, the rank one $H_{\emptyset, A_{1}, A_{2}}$ theories appear as certain limit of $\mathrm{SU}(2)$ gauge theory with $N_{f}=1,2,3$ respectively [62]. The rank one $H_{D_{4}}$ theory is well known to be the $\mathrm{SU}(2)$ gauge theory with $N_{f}=4$, while the higher rank cases with $k>1$ are equivalent to $\operatorname{USp}(2 k)$ gauge theories with four fundmental hypermultiplets and one antisymmetric hypermultiplet, which are all Lagrangian theories. The rank one $H_{E_{6,7,8}}$ are also known as the Minahan-Nemeschansky theories $[65,66]$, where the simplest example rank one $E_{6}$ theory is in S-duality with $\mathrm{SU}(3)$, $N_{f}=6$ theory [67].

All $H_{G}^{(k)}$ theory can be coupled with a free hypermultiplet associated to the center of mass motion of the instantons. We follow [37] and denote these theories as $\widetilde{H}_{G}^{(k)}$. As was observed in [45], for higher rank cases, $\widetilde{H}_{G}$ are sometimes more natural than $H_{G}$ theories. One major difference between rank one and higher rank $H_{G}$ theories is the flavour symmetry. Besides the flavour $G$ given by the strings stretched between D3-branes and exotic seven-brane, for $k>1$ there is one more $\mathrm{SU}(2)$ symmetry coming from the transverse space in the seven-brane. By coupling a free hypermultiplet, all $\widetilde{H}_{G}^{(k)}$ theories share flavour symmetry $G \times \mathrm{SU}(2)$.

\footnotetext{
${ }^{33}$ The $L_{G}$ function in [37] becomes $L_{G}^{(1)}$ here.

${ }^{34}$ The $G=\emptyset, A_{1}, A_{2}$ type theories are also traditionally denoted as $H_{0,1,2}$ theories. Here we follow the notations in [37].
} 
The $H_{G}^{(k)}$ theories of interest in this paper are $G=A_{2}, D_{4}, E_{6,7,8}$ as they are directly related to $6 \mathrm{~d}$ minimal $(1,0) \mathrm{SCFTs}$ with corresponding gauge group $G$. To be precise, the RR elliptic genus is identified as the $\beta$-twisted $T^{2} \times S^{2}$ partition function of the $4 \mathrm{~d}$ SCFTs:

$$
\mathbb{E}_{h_{G}^{(k)}}=Z_{\left(T^{2} \times S^{2}\right)_{\beta}}\left(H_{G}^{(k)}\right) .
$$

Adding the "tildes", one can also obtain the equality with the free hypermultiplet coupled. Here the $\beta$-twist was introduced by Kapustin in [68] to preserve half of the supersymmetries on the backgrounds such as $T^{2} \times S^{2}$. See a good description of such twist in for example section 3.2 of [37]. The identification (5.1) makes it sometimes possible to compute the elliptic genus from $4 \mathrm{~d}$ setting, in which cases the S-duality with a Lagrangian theory is invoked and one can use certain analogy of Spiridonov-Warnaar inverse formula [69] to compute the $T^{2} \times S^{2}$ partition function. This was indeed achieved for one string elliptic genus with $G=D_{4}, E_{6,7}[37,46-48]$. For example, the elliptic genus of one $E_{7}$ instanton string was obtained in [48] via $\mathrm{SU}(4)$ gauge theory $N_{f}=8$ and appropriate Higgsing as

$$
\begin{aligned}
Z_{\left(T^{2} \times S^{2}\right)_{\beta}}\left(H_{E_{7}}^{(1)}\right)=1 & +\chi_{133}^{E_{7}} v^{2}+\chi_{7371}^{E_{7}} v^{4}+\chi_{238602}^{E_{7}} v^{6}+\chi_{5248750}^{E_{7}} v^{8}+\ldots \\
+ & Q_{\tau}\left(1+\chi_{133}^{E_{7}}+\left(1+2 \chi_{133}^{E_{7}}+\chi_{7331}^{E_{7}}+\chi_{8645}^{E_{7}}\right) v^{2}\right. \\
& \left.\quad+\left(\chi_{133}^{E_{7}}+2 \chi_{7371}^{E_{7}}+\chi_{8645}^{E_{7}}+\chi_{238602}^{E_{7}}+\chi_{573440}^{E_{7}}\right) v^{4}+\ldots\right) \\
+ & Q_{\tau}^{2}\left(3+2 \chi_{133}^{E_{7}}+\chi_{1539}^{E_{7}}+\chi_{7371}^{E_{7}}+\ldots\right)+\mathcal{O}\left(Q_{\tau}^{3}\right)
\end{aligned}
$$

which completely agrees with our universal expansion formula (4.5), (4.6) and (4.7). ${ }^{35}$ We also checked for $D_{4}$ and $E_{6}$, where the agreement holds to all known orders.

Another important feature of $H_{G}^{(k)}$ theories is that they all admit $6 \mathrm{~d}$ construction. It is well known all rank $k H_{D_{4}, E_{6,7,8}}$ theories can be realized by compactifying a $6 \mathrm{~d} A_{N-1}$ $(2,0)$ SCFT on some punctured sphere with regular singularities [70], i.e. they are class $\mathcal{S}$ theories. The regular singularities are classified by embeddings of $\mathrm{SU}(2)$ in $\mathrm{SU}(N)$, thus can be denoted as Young diagrams. Such punctures with associated Young diagram represent how the $\mathrm{SU}(N)$ decomposes and what is the residual flavour symmetry. For example, the rank one $H_{\mathrm{SO}(8)}$ theory is obtained by compactifying $6 \mathrm{~d} A_{1}(2,0)$ SCFT on a sphere with four full punctures $\left\{1^{2}\right\}$, i.e. the residual flavour symmetry is $\mathrm{SU}(2)$. Thus the resulting $4 \mathrm{~d}$ theory has gauge symmetry $\mathrm{SU}(2)$ and four fundamentals, as was mentioned already above. We summarize the gauge algebras and punctures for the $6 \mathrm{~d}$ construction of all $H_{G}^{(k)}$ theories with $G=D_{4}, E_{6,7,8}$ in table 12 . The 6 d construction for rank $k H_{A_{2}}$ theories however involves irregular punctures. For example, they can be realized by compactifying $6 \mathrm{~d} A_{2 k-1}$ theory on a sphere with one regular puncture with Young diagram $\left\{k^{2}\right\}$ and one irregular puncture of form

$$
\Phi=\frac{1}{z^{3}} \operatorname{diag}\left(1, \ldots, 1_{k_{\mathrm{th}}},-1, \ldots,-1_{k_{\mathrm{th}}}\right)+\ldots
$$

\footnotetext{
${ }^{35}$ In the coefficients of $Q_{\tau}^{2}$, one also need to use the Joseph relation $\mathrm{Sym}^{2} \mathbf{1 3 3}=1+\mathbf{1 3 3}+\mathbf{7 3 7 1}$ to obtain the identification.
} 


\begin{tabular}{|c|c|c|}
\hline$G$ & $6 \mathrm{~d}(2,0) A_{N-1}$ & punctures $\Lambda_{i}$ \\
\hline$D_{4}$ & $A_{2 k-1}$ & four $\left\{k^{2}\right\}$ \\
\hline$E_{6}$ & $A_{3 k-1}$ & three $\left\{k^{3}\right\}$ \\
\hline$E_{7}$ & $A_{4 k-1}$ & $\left\{(2 k)^{2}\right\}$ and two $\left\{k^{4}\right\}$ \\
\hline$E_{8}$ & $A_{6 k-1}$ & $\left\{(3 k)^{2}\right\},\left\{(2 k)^{3}\right\}$ and $\left\{k^{6}\right\}$ \\
\hline
\end{tabular}

Table 12. 6d construction for rank $k H_{G}$ theory.

where the coefficients of $z^{-2}$ and $z^{-1}$ have the same type of matrix [71]. In particular, the rank one $H_{A_{2}}$ theory coincides with $\left(A_{1}, D_{4}\right)$ Argyres-Douglas theory. See also the $6 \mathrm{~d}$ construction involving irregular punctures in [72].

Class $\mathcal{S} 4 \mathrm{~d}$ SCFTs are also known to be connected to 2 d vertex operator algebra, i.e. chiral algebra [73, 74]. This correspondence relies directly on the class $\mathcal{S}$ construction and can be understood from certain generalized TQFT structure on the punctured Riemann surface. This relation sometimes gives a new approach to compute the indices of $4 \mathrm{~d}$ SCFT by realizing them as the vacuum character of associated chiral algebra. For example, the chiral algebras associated to rank one $H_{D_{4}}$ and $H_{E_{6}}$ theories are identified as $\mathfrak{s o}(8)$ affine Lie algebra at level $k_{2 d}=-2$ and $\mathfrak{e}(6)$ affine Lie algebra at level $k_{2 d}=-3$ in [73]. See some recent works trying to explain VOA/SCFT correspondence [75-79]. Besides, the rank one $H_{D_{4}, E_{6}, E_{7}}$ theories are also connected with the curved $\beta \gamma$ systems on cones over the complex Grassmannian $\operatorname{Gr}(2,4)$, the complex orthogonal Grassmannian $\mathrm{OG}^{+}(5,10)$, and the complex Cayley plane $\mathbb{O P}^{2}$ respectively in [80].

\subsection{Hall-Littlewood and Schur indices}

The superconformal index of $4 \mathrm{~d} \mathcal{N}=2 \mathrm{SCFT}$ is defined as $[81,82]$

$$
\mathcal{I}(p, q, t)=\operatorname{Tr}(-1)^{F}\left(\frac{t}{p q}\right)^{r} p^{j_{12}} q^{j_{34}} t^{R} \prod_{i} a_{i}^{f_{i}},
$$

where $j_{12}=j_{2}+j_{1}$ and $j_{34}=j_{2}-j_{1}$ denote the rotation generators in $\mathbb{C}^{2}$ with $j_{1,2}$ representing each $\mathrm{SU}(2)$ Lorentz symmetry, and $r$ and $R$ denote the $\mathrm{U}(1)_{r}$ and $\mathrm{SU}(2)_{R}$ generators respectively. Besides, $a_{i}$ are the fugacities for the flavour generators $f_{i}$ which sometimes are set to be zero for simplicity. For generic 4d SCFT, the full superconformal indices with $(p, q, t)$ are difficult to compute. For example, among all $H_{G}^{(k)}$ theories, the full superconformal indices to our knowledge are only computable so far for $H_{\mathrm{SO}(8)}$ with arbitrary rank owing to their Lagrangian nature and $H_{E_{6}, E_{7}}$ for rank one owing to the existence of certain $\mathcal{N}=1$ Lagrangian flow [47, 48].

Certain limits of superconformal index are particularly interesting due to symmetry enhancement. The name of limit comes from the observation that the resulting indices involve corresponding symmetric polynomial known in mathematics literature. Following [44], we list three of them here:

- (Macdonald) $p \rightarrow 0$. Superconformal index when taking the Macdonald limit is computable for all class $\mathcal{S}$ theory with regular punctures. For a genus $\mathfrak{g}$ theory with 
$s$ punctures compactified from $6 \mathrm{~d} A_{N-1}(2,0) \mathrm{SCFT}$, the Macdonald index is given in $[44]$ as

$$
\mathcal{I}_{\mathfrak{g}, s}^{\mathrm{M}}(\mathbf{a}, q, t)=\prod_{j=2}^{N}\left(t^{j} ; q\right)^{2 \mathfrak{g}-2+s} \frac{(t ; q)^{(k-1)(1-\mathfrak{g})+s}}{(q ; q)^{(k-1)(1-\mathfrak{g})}} \sum_{\lambda} \frac{\prod_{i=1}^{s} \hat{\mathcal{K}}_{\Lambda_{i}}\left(\mathbf{a}_{i}\right) P^{\lambda}\left(\mathbf{a}_{i}\left(\Lambda_{i}\right) \mid q, t\right)}{\left[P^{\lambda}\left(t^{\frac{k-1}{2}}, t^{\frac{k-3}{2}}, \ldots, t^{\frac{1-k}{2}} \mid q, t\right)\right]^{2 \mathfrak{g}-2+s}}
$$

Here $P^{\lambda}\left(\mathbf{a}_{i}\left(\Lambda_{i}\right) \mid q, t\right)$ are Macdonald polynomials and the summation is over all possible Young diagrams $\lambda=\left\{\lambda_{1}, \lambda_{2}, \ldots, \lambda_{N-1}, 0\right\}$. The Pochhammer symbol $(a ; b)$ is defined by

$$
(a ; b)=\prod_{i=0}^{\infty}\left(1-a b^{i}\right)
$$

The $\hat{\mathcal{K}}_{\Lambda_{i}}$ factors are defined by

$$
\hat{\mathcal{K}}_{\Lambda}(\mathbf{a})=\prod_{i=1}^{\operatorname{row}(\Lambda)} \prod_{j, k=1}^{l_{i}} \operatorname{PE}\left[\frac{a_{j}^{i} \bar{a}_{k}^{i}}{1-q}\right]_{\mathfrak{a}_{i}, q},
$$

with the coefficients $a_{k}^{i}$ associated to the Young diagram as

$$
a_{j}^{i}=c_{j} v^{\lambda_{j}+1-i} \quad \text { and } \quad \bar{a}_{k}^{i}=c_{k}^{-1} v^{\lambda_{k}+1-i},
$$

with $v^{2}=t$. Here these $c_{j}$ parameterize the residual flavour symmetry and are subject to constraint $\prod_{i=1}^{r o w(\Lambda)} \prod_{j}^{l_{i}} c_{j}=1$ to preserve the traceless condition of $\mathrm{SU}(N)$. The association of the flavour fugacities for a puncture $a(\Lambda)$ in Macdonald polynomial is defined similarly as $c_{j} v^{-\lambda_{j}-1+2 i}$. Some good figures to visualize these definitions can be found in $[44,45]$.

- (Hall-Littlewood) $p, q \rightarrow 0$. By taking limit in (5.5), it is easy to obtain the HallLittlewood index for all class $\mathcal{S}$ theories. As only genus zero theories are of concern in this paper, we only write down the formulas with $\mathfrak{g}=0$. For example, the HallLittlewood index of $4 \mathrm{~d}$ SCFT compactified from $6 \mathrm{~d} A_{N-1}$ theory is

$$
\mathcal{I}^{\mathrm{HL}}=\mathcal{N}_{N, s} \sum_{\lambda} \frac{\prod_{i=1}^{s} \hat{\mathcal{K}}_{\Lambda_{i}}\left(\mathbf{a}_{i}\right) \psi^{\lambda}\left(\mathbf{a}_{i}\left(\Lambda_{i}\right) \mid v\right)}{\left[\psi^{\lambda}\left(v^{N-1}, v^{N-3}, \ldots, v^{1-N} \mid v\right)\right]^{s-2}},
$$

where

$$
\mathcal{N}_{N, s}=\left(1-v^{2}\right)^{N-1+s} \prod_{j=2}^{N}\left(1-v^{2 j}\right)^{s-2},
$$

and $\psi^{\lambda}$ is the Hall-Littlewood polynomials defined as

$$
\psi^{\lambda}\left(x_{1}, \ldots, x_{N} \mid v\right)=\mathcal{N}_{\lambda}(v) \sum_{\sigma \in S_{N}} x_{\sigma(1)}^{\lambda_{1}} \ldots x_{\sigma(N)}^{\lambda_{N}} \prod_{i<j} \frac{x_{\sigma(i)}-v^{2} x_{\sigma(j)}}{x_{\sigma(i)}-x_{\sigma(j)}},
$$

with

$$
\mathcal{N}_{\lambda}(v)=\prod_{i=0}^{\infty} \prod_{j=1}^{m(i)}\left(\frac{1-v^{2 j}}{1-v^{2}}\right)^{-1 / 2}
$$


where $m(i)$ is the number of rows in the Young diagram $\lambda=\left(\lambda_{1}, \ldots, \lambda_{N}\right)$ of length $i$. Here we have made the substitution $t=v^{2}$ for convenience.

It is argued in [44] that for linear quiver theories the HL index is equivalent to the Hilbert series of the Higgs branch. In particular, this is true for all $H_{G}$ theories. It is well-known the Higgs branch of $H_{G}^{(k)}$ theories are the reduced moduli space of $k G$-instantons, which can be understood from the probing picture that the $k$ D3branes dissolving into the seven-branes resemble $k$ instantons in the transverse space. Thus the HL index of $H_{G}^{(k)}$ theory are supposed to be equal to the Hilbert series of reduced moduli space of $k G$-instantons. On the other hand, the Hilbert series can also be obtained from the $5 \mathrm{~d}$ Nekrasov partition function with pure gauge group $G$, which are just the $5 \mathrm{~d}$ limit of elliptic genus of $6 \mathrm{~d}$ minimal $(1,0)$ SCFT with type $G$. Therefore, we arrive at the relation:

$$
\mathcal{I}_{H_{G}^{(k)}}^{\mathrm{HL}}=\operatorname{Hilb}_{G}^{k}=g_{k, G}^{(0)},
$$

where $g_{k, G}^{(0)}$ as we defined previously in (4.3) is the coefficient of leading $Q_{\tau}$ order of $k$-string elliptic genus $\mathbb{E}_{h_{G}^{(k)}}$. One can also add "tildes" to get the equality with a free hypermultiplet coupled, in which situation one encounters the full Hilbert series other than the reduced. We have checked relation (5.13) for $k=1,2$ for all possible $G$ and $k=3$ for $\mathrm{SU}(3) \cdot{ }^{36}$

- (Schur) $q=t$ with $p$ arbitrary. In fact, it can be shown in such specialization the index is independent of $p$. Thus, taking $p \rightarrow 0$, Schur index is actually a limit of Macdonald index. Using (5.5), the Schur index for a class $\mathcal{S}$ theory is given by

$$
\mathcal{I}^{\text {Schur }}=\hat{\mathcal{N}}_{N, s} \frac{\prod_{i=1}^{s} \hat{\mathcal{K}}_{\Lambda_{i}}\left(\mathbf{a}_{i}\right) \chi^{\lambda}\left(\mathbf{a}_{i}\left(\Lambda_{i}\right)\right)}{\left[\chi^{\lambda}\left(v^{N-1}, v^{N-3}, \ldots, v^{1-N}\right)\right]^{s-2}},
$$

where $^{37}$

$$
\hat{\mathcal{N}}_{N, s}=\left(v^{2} ; v^{2}\right)^{s} \prod_{j=2}^{N}\left(v^{2 j} ; v^{2}\right)^{s-2},
$$

and $\chi^{\lambda}$ is the Schur polynomials defined as

$$
\chi_{\lambda}(\mathbf{a})=\frac{\operatorname{det}\left(a_{i}^{\lambda_{j}+k-j}\right)}{\operatorname{det}\left(a_{i}^{k-j}\right)} .
$$

At last, one replaces back $v^{2} \rightarrow q$.

The Schur indices in some sense are more interesting than the Hall-Littlewood indices. For instance, for class $\mathcal{S}$ theories, Schur indices equal the $q$-deformed topological $2 \mathrm{~d}$

\footnotetext{
${ }^{36}$ For $\mathrm{SU}(3)$ and $F_{4}$, we are not aware how to compute the HL indices directly. Still, the Hilbert series are well-defined and computed in [41,42], which are in perfect agreement with our computation for elliptic genus from blowup equations.

${ }^{37}$ As in this paper we only deal with the cases with three or four punctures, we also shorten $\mathcal{N}_{N, 3}$ as $\mathcal{N}_{N}$ and $\mathcal{N}_{N, 4}$ as $\mathcal{N}_{N}^{\prime}$ in the latter subsections, and same for those with hat.
} 
Yang-Mills partition function on the punctured Riemann surface [83], and also equal the vacuum character of the associated chiral algebra [73, 74]. Furthermore, Schur indices can be computed in IR via wall crossing for theories even beyond class $\mathcal{S}$, such as certain Argyres-Douglas theories [84] including rank one $H_{A_{2}}$ theory.

The full superconformal indices of rank one $H_{D_{4}, E_{6,7}}$ theories have been computed in $[46,48,85]$. The Schur index of rank one $H_{E_{8}}$ was given in [37] and the Schur index of rank one $H_{A_{2}}$ was given in [84]. To compute the Hall-Littlewood indices and Schur indices of higher rank $H_{D_{4}, E_{6,7,8}}$ theories one will encounter certain subtle issues. Directly using the general formulas (5.9) and (5.14) fails to give correct results, because at a given order of $v$ infinite number of Young diagrams $\lambda$ contribute. To cure such divergence, it was suggested in [45] that one reduces the flavor symmetry "one box at a time", that is to change one specific puncture by moving one box down in the associated Young diagram. The physical meaning of such operation is interpreted as coupling a free hypermultiplet to $H_{G}^{(k)}$ theory, which in our notation is just $\widetilde{H}_{G}^{(k)}$ theory. In the terminology of [45], $H_{G}^{(k)}$ are "bad" theories, while $\widetilde{H}_{G}^{(k)}$ are "good" theories. One can directly use (5.9) and (5.14) to compute the indices of $\widetilde{H}_{G}^{(k)}$, then divide by the index of a free hypermultiplet which is well defined, finally one will obtain the finite indices of $H_{G}^{(k)}$. Following this procedure, the Hall-Littlewood indices of rank two $H_{D_{4}, E_{6,7,8}}$ theories were computed in [45]. Similarly, we computed the Schur indices of rank two and three $H_{D_{4}, E_{6,7,8}}$ theories which will be shown in details in later sections. For higher rank $H_{A_{2}}$ we are not aware how to compute its Schur indices due to the irregular punctures of $6 \mathrm{~d}$ construction. Although there exist no $H_{G}^{k}$ theory for $G=F_{4}$, we suspect certain analogy can be constructed such that HallLittlewood indices still make sense as the Hilbert series of moduli space of $k F_{4}$ instantons, and the Schur indices can be associated with affine $\mathfrak{f}_{4}$ algebra. One support for such speculation is that the Hilbert series for arbitrary $k F_{4}$ instantons has been constructed from certain folding from $E_{6}$ [43]. Thus we sometimes informally denote the analogy as $H_{F_{4}}^{(k)}$ theories.

\subsection{Rank one: Del Zotto-Lockhart's conjecture}

In [37], Del Zotto-Lockhart found an intriguing structure of one string elliptic genera of 6d mininal $(1,0)$ SCFTs and a surprising relation between the elliptic genera and the supersymmetric indices of rank one $H_{G}$ theories. Let us rephrase their conjecture here:

Conjecture (Del Zotto-Lockhart). There exists a function $L_{G}^{(1)}\left(v, m_{G}, Q_{\tau}\right)=\sum_{i, j=0}^{\infty} b_{i, j}^{G} Q_{\tau}^{i} v^{j}$ such that

1. $b_{i, j}^{G}$ can be written as the sum of characters of irreducible representations of $G$ with integral coefficients.

2. $L_{G}^{(1)}\left(v, m_{G}, 0\right)$ is the Hilbert series of the reduced moduli space of one $G$-instanton, i.e. the Hall-Littlewood index of the $H_{G}^{(1)}$ theory.

3. $L_{G}^{(1)}\left(q^{1 / 2}, m_{G}, q^{2}\right)$ is the Schur index of the $H_{G}^{(1)}$ theory. 


\begin{tabular}{|c|cccccccc|}
\hline$i, j$ & 0 & 2 & 4 & 6 & 8 & 10 & 12 & 14 \\
\hline 0 & 1 & 28 & 300 & 1925 & 8918 & 32928 & 102816 & 282150 \\
1 & 0 & 0 & 29 & 707 & 6999 & 42889 & 193102 & 699762 \\
2 & -1 & 0 & 0 & $2 \cdot 1$ & $463+1$ & 9947 & 92391 & 544786 \\
3 & 0 & -28 & -29 & $-2 \cdot 1$ & $1-1$ & $2 \cdot 29$ & $5280+29+2 \cdot 28$ & 101850 \\
4 & 0 & 0 & -300 & -707 & $-463-1$ & $-2 \cdot 29$ & $29-29$ & $2 \cdot 463+2 \cdot 1$ \\
5 & 0 & 0 & 0 & -1925 & -6999 & -9947 & $-5280-29-2 \cdot 28$ & $-2 \cdot 463-2 \cdot 1$ \\
6 & 0 & 0 & 0 & 0 & -8918 & -42889 & -92391 & -101850 \\
7 & 0 & 0 & 0 & 0 & 0 & -32928 & -193102 & -544786 \\
8 & 0 & 0 & 0 & 0 & 0 & 0 & -102816 & -699762 \\
9 & 0 & 0 & 0 & 0 & 0 & 0 & 0 & -282150 \\
\hline
\end{tabular}

Table 13. Expansion coefficients $c_{i, j}^{\mathrm{SO}(8)}$ for one $\mathrm{SO}(8)$ instanton string.

4. The reduced one-string elliptic genus $\mathbb{E}_{h_{G}^{(1)}}(v)$ can be generated from $L_{G}^{(1)}(v)$ by the following formula in which the symmetry $(4.26)$ is manifest: ${ }^{38}$

$$
\begin{aligned}
\mathbb{E}_{h_{G}^{(1)}}(v)= & v^{2 h-1} Q_{\tau}^{1 / 6} \sum_{n \geq 0} Q_{\tau}^{2 n}\left[u^{4 h} L_{G}\left(Q_{\tau}^{n} v\right)-(-1)^{2 h} u^{-4 h} L_{G}\left(Q_{\tau}^{n+1 / 2} / v\right)\right. \\
& +\left(1+(-1)^{2 h}\right) Q_{\tau}^{h+1 / 2}\left(u^{2} L_{G}\left(Q_{\tau}^{n+1 / 2} v\right)-u^{-2} L_{G}\left(Q_{\tau}^{n+1} / v\right)\right) \\
& \left.+Q_{\tau}^{2}\left((-1)^{2 h} u^{4(1-h)} L_{G}\left(Q_{\tau}^{n+1} v\right)-u^{-4(1-h)} L_{G}\left(Q_{\tau}^{n+3 / 2} / v\right)\right)\right]
\end{aligned}
$$

where $h=h_{G}^{\vee} / 6, u=v / Q_{\tau}^{1 / 4}$.

The conjectural formula (5.17) is quite intricate. Roughly speaking, it means the coefficient matrix of reduced one-string elliptic genus contains several "blocks", overlapping or non-overlapping, and each block contains infinite copies of the $L_{G}^{(1)}$ function. The number of blocks turns out to be 2 for $\mathrm{SU}(3), 4$ for $F_{4}$ and 6 for the other $G$. In the following we show the coefficient matrix of one-string elliptic genus of $\mathrm{SO}(8)$ in a way consistent with our later higher rank discussion. The coefficient matrix of elliptic genus and the $L_{G}^{(1)}$ functions for other $G$ can be found in [37]. Let us denote

$$
\mathbb{E}_{h_{\mathrm{SO}(8)}^{(1)}}\left(v, Q_{\tau}, m_{i}=0\right)=v^{5} Q_{\tau}^{-5 / 6} \sum_{i, j=0}^{\infty} c_{i, j}^{\mathrm{SO}(8)} v^{j}\left(Q_{\tau} v^{-4}\right)^{i} .
$$

Then we have table 13 for the coefficients $c_{i, j}^{\mathrm{SO}(8)}$ where each "block" is colored differently: the coefficients coming from the first term in the square bracket in (5.17) is colored red, the second black, the third blue, the forth orange, the fifth cyan and the last magenta. As we can see from the table, the reduced one-string elliptic genus indeed depends on $v^{2}$. One can also see the symmetry (4.26) on the two sides of the ray with slop $-1 / 2$. Here the

\footnotetext{
${ }^{38}$ Here the dependence on $Q_{\tau}$ and $Q_{m}$ are implied.
} 
$L_{\mathrm{SO}(8)}^{(1)}\left(v, Q_{\tau}\right)$ function can be defined by all the red numbers in table 13 with the red +1 and +29 moving out, as they come from $n=1$ term in the summation. Thus we have

$$
\begin{aligned}
L_{\mathrm{SO}(8)}^{(1)} & \left(v, Q_{\tau}\right) \\
= & \left(1+28 v^{2}+300 v^{4}+1925 v^{6}+8918 v^{8}+32928 v^{10}+102816 v^{12}+282150 v^{14}+\mathcal{O}\left(v^{16}\right)\right) \\
& +\left(29+707 v^{2}+6999 v^{4}+42889 v^{6}+193102 v^{8}+699762 v^{10}+\mathcal{O}\left(v^{12}\right)\right) Q_{\tau} \\
& +\left(463+9947 v^{2}+92391 v^{4}+544786 v^{6}+\mathcal{O}\left(v^{8}\right)\right) Q_{\tau}^{2} \\
& +\left(5280+101850 v^{2}++\mathcal{O}\left(v^{4}\right)\right) Q_{\tau}^{3}+\mathcal{O}\left(Q_{\tau}^{4}\right) .
\end{aligned}
$$

Clearly, the first row in table 13 gives the well-known Hilbert series for the reduced moduli space for one $\mathrm{SO}(8)$ instanton, i.e. the Hall-Littlewood index for rank one $H_{\mathrm{SO}(8)}$ theory:

$$
L_{\mathrm{SO}(8)}^{(1)}(v, 0)=\sum_{n=0}^{\infty} \chi_{n \theta}^{\mathrm{SO}(8)} v^{2 n}=1+28 v^{2}+300 v^{4}+1925 v^{6}+8918 v^{8}+32928 v^{10}+102816 v^{12}+\mathcal{O}\left(v^{14}\right)
$$

Adding the red numbers from $L_{\mathrm{SO}(8)}^{(1)}\left(v, Q_{\tau}\right)$ in each column of table 13 together, one expects to obtain the Schur index of rank one $H_{\mathrm{SO}(8)}$ theory. Indeed, by making $v \rightarrow q^{1 / 2}$ to make contact with the literature, we obtain

$$
L_{\mathrm{SO}(8)}\left(q^{1 / 2}, q^{2}\right)=1+28 q+329 q^{2}+2632 q^{3}+16380 q^{4}+85764 q^{5}+393589 q^{6}+1628548 q^{7}+\mathcal{O}\left(q^{8}\right) .
$$

Such series was actually already obtained by a lot of methods. For example, from the viewpoint of VOA/SCFT correspondece, it equals the vacuum character of affine Lie algebra $\mathfrak{s o}(8)_{k=-2}[73]$. From the nature that rank one $H_{\mathrm{SO}(8)}$ theory is actually just $\mathrm{SU}(2)$ gauge theory with $N_{f}=4$, the Schur index can be computed both from UV Lagrangian and IR wall-crossing formula [86]. See the Schur series from vacuum character up to $q^{14}$ in the end of the appendix of [86].

Such comparison between the reduced elliptic genus and Schur index for all other rank one $H_{G}$ theory except $G=F_{4}$ has been done in [37]. In particular, all $L_{G}^{(1)}\left(v, Q_{\tau}, m_{G}=0\right)$ functions are identified, and the conjectural formula (5.17) holds to substantial orders. Similarly, one can also couple a free hypermultiplet to establish the relation between original one-string elliptic genus $\mathbb{E}_{\tilde{h}_{G}^{(1)}}(v)$ and the Hall-Littlewood and Schur indices of $\widetilde{H}_{G}^{(k)}$ theory. Indeed, the Schur index of a $4 \mathrm{~d}$ hypermultiplet is known to be [86]

$$
\mathcal{I}_{h . m .}^{\text {Schur }}=\operatorname{PE}\left[\frac{q^{1 / 2}}{1-q}\left(x+x^{-1}\right)\right]
$$

which can also be obtained by taking limit $\mathbb{E}_{\text {c.m. }}\left(v, x, Q_{\tau}\right) \rightarrow \mathbb{E}_{\mathrm{cm}}\left(q^{1 / 2}, x, q^{2}\right)$. The HallLittlewood index of a $4 \mathrm{~d}$ hypermultiplet i.e. the Hilbert series of $\mathbb{C}^{2}$ is well-known to be

$$
\mathcal{I}_{h . m .}^{\mathrm{HL}}=\frac{1}{\left(1-v x^{ \pm 1}\right)},
$$

which can also be obviously obtained by taking limit $\mathbb{E}_{\text {c.m. }}\left(v, x, Q_{\tau} \rightarrow 0\right)$, with a factor $v Q_{\tau}^{-1 / 6}$ absorbed into the overall factor of (4.3). This makes the whole story consistent. 
In the viewpoint of pure $4 \mathrm{~d}$, this intriguing conjecture indicates there exists certain precise relation between the $\beta$-twisted partition function on $T^{2} \times S^{2}$ and the partition function on $S^{3} \times S^{1}$. We suspect the connection may be established by realizing one $S^{1}$ of $T^{2}$ as the Hopf fibration over $S^{2}$ to get $S^{3} \times S^{1}$. To find the consequence of such realization one has to go into the details of localization which is beyond the scope of current paper. ${ }^{39}$

\subsection{Rank two}

We would like to generalize Del Zotto-Lockhart's conjecture to the rank two cases, where there exist more flavour symmetry that is $\mathrm{SU}(2)_{x}$ in $H_{G}$ theories. To be precise, we want to find some functions $L_{G}^{(2)}\left(v, x, m_{G}, Q_{\tau}\right)=\sum_{i, j=0}^{\infty} b_{i, j}^{G} Q_{\tau}^{i} v^{j}$ such that

1. $b_{i, j}^{G}$ can be written as the sum of products between the characters of irreducible representations of $\mathrm{SU}(2)_{x}$ and the characters of irreducible representations of $G$ with integral coefficients.

2. $L_{G}^{(2)}\left(v, x, m_{G}, 0\right)$ is the Hilbert series of the reduced moduli space of two $G$-instanton, i.e. the Hall-Littlewood index of the $H_{G}^{(2)}$ theory.

3. $L_{G}^{(2)}\left(q^{1 / 2}, x, m_{G}, q^{2}\right)$ is the Schur index of the $H_{G}^{(2)}$ theory.

4. The reduced two-string elliptic genus $\mathbb{E}_{h_{G}^{(2)}}\left(v, x, m_{G}, Q_{\tau}\right)$ can be generated from $L_{G}^{(2)}\left(v, x, m_{G}, Q_{\tau}\right)$ and $L_{G}^{(1)}\left(v, x, m_{G}, Q_{\tau}\right)$ functions.

It turns out the rank two cases are much more complicated than the rank one cases, one reason for which is that we can not rely on the additional symmetry (4.27). Although we have not achieved an exact formula to generate the two string elliptic genus, we successfully manage to identify the $L_{G}^{(2)}$ functions to substantial orders, which we will elaborate on later for each example. In fact, the leading and subleading $Q_{\tau}$ order of $L_{G}^{(2)}\left(v, x, m_{G}, Q_{\tau}\right)$ are just given by $g_{2, G}^{(0)}\left(v, x, m_{G}\right)$ in (4.8) and $g_{2, G}^{(1)}\left(v, x, m_{G}\right)$ in (4.9), while the subsubleading order is given by

$$
\left(\chi_{5}+\left(\chi_{\theta}+2\right) \chi_{3}+\chi_{\operatorname{Sym}^{2} \theta}+2 \chi_{\theta}+3\right)+\left(\left(\chi_{\theta}+1\right) \chi_{4}+\left(\left(\chi_{\theta}+1\right)^{2}+\left(2 \chi_{\theta}+1\right)\right) \chi_{2}\right) v+\ldots,
$$

which differs from $g_{2, G}^{(2)}\left(v, x, m_{G}\right)$ in (4.10) by $1+\chi_{2} v+\ldots$. Such difference is recognized as what we call "blue" series in contrast to the red $L_{G}^{(2)}$ functions. Indeed, the reason we also include $L_{G}^{(1)}$ in the last condition is that we observe a "blue" series appearing multiple times in the coefficient matrix of $\mathbb{E}_{h_{G}^{(2)}}$ :

$$
\begin{aligned}
& M_{G}^{(2), \text { blue }}(v, x)=\sum_{n=0}^{\infty} v^{n} \sum_{i+2 j=n+1} \chi_{i} \chi_{j \theta}=\frac{1}{(1-v x)(1-v / x)} g_{1, G}^{(0)}(v) \\
& =1+\chi_{2} v+\left(\chi_{3}+\chi_{\theta}\right) v^{2}+\left(\chi_{4}+\chi_{\theta} \chi_{2}\right) v^{3}+\left(\chi_{5}+\chi_{\theta} \chi_{3}+\chi_{2 \theta}\right) v^{4}+\left(\chi_{6}+\chi_{\theta} \chi_{4}+\chi_{2 \theta} \chi_{2}\right) v^{5}+\ldots
\end{aligned}
$$

\footnotetext{
${ }^{39}$ Guglielmo Lockhart came up independently with a similar idea (private communication).
} 


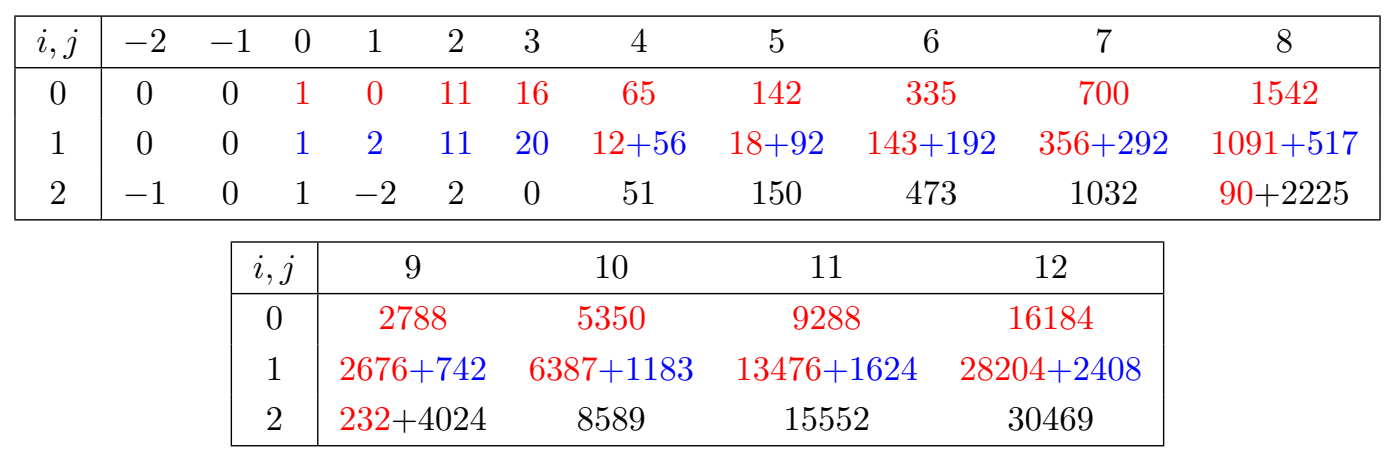

Table 14. Unrefined coefficients $c_{i, j}^{A_{2}}$ for the elliptic genus of two SU(3) instanton strings.

For example, the blue series always appears at $Q_{\tau}$ order $h_{G}^{\vee} / 3$ with leading $v$ order $-2 h_{G}^{\vee} / 3$ (comparing to the leading $Q_{\tau}$ order). The reason for such phenomenon is yet not clear to us.

On the other hand, from the technique of class $\mathcal{S}$ theory, we can compute the Schur index of $H_{G}^{(2)}$ theories for $G=D_{4}, E_{6,7,8}$. All of them are in agreement with our expectation from elliptic genera up to quite high orders. For example, from the $L_{G}^{(2)}$ functions, we are able to write down the following general formula for the Schur indices up to $q^{7 / 2}$ :

$$
\begin{aligned}
\mathcal{I}_{H_{G}^{(2)}}^{\text {Schur }}= & \mathcal{I}_{\widetilde{H}_{G}^{(2)}}^{\text {Schur }} / \mathcal{I}_{h . m .}^{\text {Schur }}=1+\left(\chi_{3}+\chi_{\theta}\right) q+\chi_{\theta} \chi_{2} q^{3 / 2}+\left(\chi_{5}+\left(\chi_{\theta}+1\right) \chi_{3}+\chi_{\mathrm{Sym}^{2} \theta}+\chi_{\theta}+1\right) q^{2} \\
& +\left(\chi_{\theta} \chi_{4}+\left(\chi_{2 \theta}+\chi_{\mathrm{Sym}^{2} \theta}+1\right) \chi_{2}\right) q^{5 / 2}+\left(\chi_{7}+\left(\chi_{\theta}+1\right) \chi_{5}+\left(\chi_{2 \theta}+\chi_{\mathrm{Sym}^{2} \theta}+2 \chi_{\theta}+3\right) \chi_{3}\right. \\
& \left.+\chi_{\mathrm{Sym}^{3} \theta}+\left(\chi_{\theta}+1\right)^{2}-C_{6}(G)\right) q^{3}+\left(\chi_{\theta} \chi_{6}+\left(\chi_{2 \theta}+\chi_{\mathrm{Alt}^{2} \theta}+2 \chi_{\theta}+1\right) \chi_{4}\right. \\
& \left.+\left(\chi_{3 \theta}+2 \chi_{2 \theta}+\left(\chi_{\theta}+1\right)^{2}+\chi_{\mathrm{Sym}^{2} \theta}+\chi_{\mathrm{Alt}^{2} \theta}+B_{2}(G)+C_{7}(G)\right) \chi_{2}\right) q^{7 / 2}+\ldots
\end{aligned}
$$

In the following, we show the striking comparison between elliptic genus and indices at rank two for all symmetry group $G$.

SU(3). For SU(3), let us denote the two-string elliptic genus as

$$
\mathbb{E}_{h_{A_{2}}^{(2)}}\left(v, x, Q_{\tau}, Q_{m}\right)=v^{5} Q_{\tau}^{-5 / 6} \sum_{i, j=0}^{\infty} c_{i, j}^{A_{2}}\left(x, Q_{m}\right) v^{j}\left(Q_{\tau} v^{-4}\right)^{i} .
$$

Then we have the unrefined coefficients $c_{i, j}^{A_{2}}\left(x=1, Q_{m}=1\right)$ listed in table 14 . Keeping in mind that all such numbers can be refined to incorporate $\mathrm{SU}(2)_{x}$, we show the unrefined coefficients just to make them look clearer. The red numbers give the definition of $L_{G}^{(2)}$ functions. In particular, they are in agreement with the universal expansion (4.8), (4.9) and (5.24). Note the red numbers in the first row agrees with the Hilbert series for reduced moduli space of two $A_{2}$ instantons in [42]. The two red numbers in the $i=2$ rows are predicted from (5.24). Besides, the blue numbers agree with our proposal (5.25). Adding the red numbers in each column together, we expect to obtain a series that is equal to the Schur index of rank two $H_{A_{2}}$ 4d SCFT.

The construction of $H_{A_{2}}^{(2)}$ theory from 6d involves irregular punctures. We are not aware how to directly compute its indices. We write our prediction from elliptic genus 
here: the Hall-Littlewood index of rank two $H_{A_{2}}$ theory is

$$
\begin{aligned}
\mathcal{I}_{H_{A_{2}}^{(2)}}^{\mathrm{HL}}= & 1+\left(\chi_{3}+8\right) q+8 \chi_{2} q^{3 / 2}+\left(\chi_{5}+8 \chi_{3}+36\right) q^{2}+\left(8 \chi_{4}+55 \chi_{2}\right) q^{5 / 2} \\
& +\left(\chi_{7}+8 \chi_{5}+63 \chi_{3}+119\right) q^{3}+\left(8 \chi_{6}+55 \chi_{4}+216 \chi_{2}\right) q^{7 / 2} \\
& +\left(\chi_{9}+8 \chi_{7}+63 \chi_{5}+280 \chi_{3}+322\right) q^{4}+\left(8 \chi_{8}+55 \chi_{6}+280 \chi_{4}+637 \chi_{2}\right) q^{9 / 2}+\mathcal{O}\left(q^{5}\right)
\end{aligned}
$$

which agrees with the Hilbert series of reduced moduli space of two SU(3) instantons [42], and the Schur index of rank two $H_{A_{2}}$ theory is

$$
\begin{aligned}
\mathcal{I}_{H_{A_{2}}^{(2)}}^{\text {Schur }}= & 1+\left(\chi_{3}+8\right) q+8 \chi_{2} q^{3 / 2}+\left(\chi_{5}+9 \chi_{3}+45\right) q^{2}+\left(8 \chi_{4}+64 \chi_{2}\right) q^{5 / 2} \\
& +\left(\chi_{7}+9 \chi_{5}+82 \chi_{3}+200\right) q^{3}+\left(8 \chi_{6}+72 \chi_{4}+360 \chi_{2}\right) q^{7 / 2} \\
& +\left(\chi_{9}+9 \chi_{7}+83 \chi_{5}+479 \chi_{3}+799\right) q^{4}+\left(8 \chi_{8}+72 \chi_{6}+496 \chi_{4}+1608 \chi_{2}\right) q^{9 / 2}+\mathcal{O}\left(q^{5}\right) .
\end{aligned}
$$

Taking $x=1$ in (5.29), we have the unrefined Schur index as

$$
1+11 q+16 q^{3 / 2}+77 q^{2}+160 q^{5 / 2}+498 q^{3}+1056 q^{7 / 2}+2723 q^{4}+5696 q^{9 / 2}+\mathcal{O}\left(q^{5}\right) .
$$

This is in complete agreement with Beem-Rastalli's to appear computation from chiral algebra! ${ }^{40}$

SO(8). The $H_{D_{4}}^{(2)}$ theory can be constructed by compactifying $A_{3}(2,0) 6 \mathrm{~d}$ SCFT on a sphere with four square punctures $\left\{2^{2}\right\}$, i.e. 2222 theory, which is expected to be a $u s p(4)$ gauge theory with four fundamental hypermultiplets and one anti-fundamental. On the other hand, the $\widetilde{H}_{D_{4}}^{(2)}$ theory can be constructed as a $222 L$ theory, i.e. we replace one $\left\{2^{2}\right\}$ puncture to $\left\{2,1^{2}\right\}$. In [45], the Hall-Littlewood indices of both $222 L$ theory and $u s p(4)+4 f+1 a$ theory were computed, which are in relation

$$
\mathcal{I}_{222 L}\left(v, x, m_{i}\right)=\frac{1}{1-v x^{ \pm 1}} \mathcal{I}_{u s p(4)+4 f+1 a}\left(v, x, m_{i}\right) .
$$

We expect and indeed checked to high orders

$$
\mathcal{I}_{u s p(4)+4 f+1 a}\left(v, x, m_{i}\right)=g_{0, D_{4}}^{(2)}\left(\tau, a, m_{i}\right) .
$$

For example, one can directly see the series coefficients in (A.12) of [45] agree with the $Q_{\tau}^{0}$ entries in table 15 .

The Schur index of $222 \mathrm{~L}$ theory can be obtained in a similar manner. Following the general formula in [44], we obtain

$$
\begin{aligned}
& \mathcal{I}_{222 L}^{\text {Schur }}(c, d, e ; a, b)=\hat{\mathcal{N}}_{4}^{\prime} \hat{\mathcal{K}}_{1}(c) \hat{\mathcal{K}}_{1}(d) \hat{\mathcal{K}}_{1}(e) \hat{\mathcal{K}}_{2}(a, b) \sum_{\lambda} \frac{\chi_{\lambda}\left(v b, v^{-1} b, b^{-1} a, b^{-1} a^{-1}\right)}{\chi_{\lambda}^{2}\left(v^{-3}, v^{-1}, v, v^{3}\right)} \\
& \quad \times \chi_{\lambda}\left(v c, v^{-1} c, v c^{-1}, v^{-1} c^{-1}\right) \chi_{\lambda}\left(v d, v^{-1} d, v d^{-1}, v^{-1} d^{-1}\right) \chi_{\lambda}\left(v e, v^{-1} e, v e^{-1}, v^{-1} e^{-1}\right),
\end{aligned}
$$

\footnotetext{
${ }^{40}$ We thank Beem and Rastelli for providing us their unpublished results on the unrefined Schur index of rank two $\mathrm{SU}(3)$ theory.
} 
where $\left(b_{1}=b, b_{2}=1 / b\right)$. The summation is over Young diagrams $\lambda=\left(\lambda_{1}, \lambda_{2}, \lambda_{3}, 0\right)$. The $\hat{\mathcal{N}}$ and $\hat{\mathcal{K}}$ factors are given by

$$
\begin{aligned}
\hat{\mathcal{N}}_{4}^{\prime} & =\left(v^{2} ; v^{2}\right)^{4} \prod_{j=2}^{4}\left(v^{2 j} ; v^{2}\right)^{2}, \\
\hat{\mathcal{K}}_{1}(b) & =\operatorname{PE}\left[\frac{\left(v^{2}+v^{4}\right)\left(b^{2}+b^{-2}+2\right)}{1-v^{2}}\right], \\
\hat{\mathcal{K}}_{2}(a, b) & =\operatorname{PE}\left[\frac{3 v^{2}+v^{4}+v^{3} b^{ \pm 2} a^{ \pm 1}+v^{2} a^{ \pm 2}}{1-v^{2}}\right] .
\end{aligned}
$$

At last, one usually replaces $v \rightarrow q^{1 / 2}$ to make contact with literature. From the above formula, we computed the Schur index up to $v^{20}$ as

$$
\mathcal{I}_{222 L}^{\text {Schur }}=1+\chi_{2} v+\left(2 \chi_{3}+28\right) v^{2}+\left(2 \chi_{4}+58 \chi_{2}\right) v^{3}+\left(3 \chi_{5}+87 \chi_{3}+465\right) v^{4}+\ldots
$$

Decoupling the free hypermultiplet, we obtain the Schur index of $H_{D_{4}}^{(2)}$ theory

$$
\mathcal{I}_{H_{D_{4}}^{(2)}}^{\text {Schur }}=\mathcal{I}_{\text {usp }(4)+4 f+1 a}^{\text {Schur }}=\mathcal{I}_{222 L}^{\text {Schur }} / \mathcal{I}_{h . m}^{\text {Schur }}
$$

up to $q^{10}$. The first 12 terms with full $\mathrm{SU}(2)_{x}$ fugacity are

$$
\begin{aligned}
\mathcal{I}_{H_{D_{4}}^{(2)}}^{\text {Schur }}= & 1+\left(\chi_{3}+28\right) q+28 \chi_{2} q^{3 / 2}+\left(\chi_{5}+29 \chi_{3}+435\right) q^{2}+\left(28 \chi_{4}+707 \chi_{2}\right) q^{5 / 2} \\
& +\left(\chi_{7}+29 \chi_{5}+765 \chi_{3}+4845\right) q^{3}+\left(28 \chi_{6}+735 \chi_{4}+9947 \chi_{2}\right) q^{7 / 2} \\
& +\left(\chi_{9}+29 \chi_{7}+766 \chi_{5}+12337 \chi_{3}+43353\right) q^{4} \\
& +\left(28 \chi_{8}+735 \chi_{6}+12607 \chi_{4}+101878 \chi_{2}\right) q^{9 / 2} \\
& +\left(\chi_{11}+29 \chi_{9}+766 \chi_{7}+12667 \chi_{5}+141518 \chi_{3}+330360\right) q^{5} \\
& +\left(28 \chi_{10}+735 \chi_{8}+12635 \chi_{6}+155449 \chi_{4}+845225 \chi_{2}\right) q^{11 / 2}+\mathcal{O}\left(q^{6}\right)
\end{aligned}
$$

We can compare this with elliptic genus up to $q^{11 / 2}$. Let us denote the $\mathrm{SO}(8)$ two-string elliptic genus as

$$
\mathbb{E}_{h_{D_{4}}^{(2)}}\left(v, x, \tau, m_{i}=0\right)=v^{11} Q_{\tau}^{-11 / 6} \sum_{i, j=0}^{\infty} c_{i, j}^{\mathrm{SO}(8)}(x) v^{j}\left(Q_{\tau} v^{-4}\right)^{i} .
$$

Then we have table 15 for the coefficients $c_{i, j}^{\mathrm{SO}(8)}(x=1)$. Here the red numbers are from the $L_{D_{4}}^{(2)}$ series. Add the red numbers in each column together, we expect to obtain a series that is equal to the Schur index of rank two $H_{D_{4}} 4 \mathrm{~d}$ SCFT. Indeed, we have

$$
\begin{aligned}
L_{D_{4}}^{(2)}\left(q^{1 / 2}, x=1, m_{D_{4}}=0, q^{2}\right)= & 1+31 q+56 q^{3 / 2}+527 q^{2}+1526 q^{5 / 2}+7292 q^{3}+23002 q^{7 / 2} \\
& +84406 q^{8}+258818 q^{9 / 2}+823883 q^{5}+2394216 q^{11 / 2}+\ldots
\end{aligned}
$$

On the other hand, by taking the unrefined limit $x=1$ in (5.36), we obtain the unrefined Schur series

$$
\begin{aligned}
1 & +31 q+56 q^{3 / 2}+527 q^{2}+1526 q^{5 / 2}+7292 q^{3}+23002 q^{7 / 2}+84406 q^{8}+258818 q^{9 / 2}+823883 q^{5} \\
& +2394216 q^{11 / 2}+6943434 q^{6}+19082748 q^{13 / 2}+51665849 q^{7}+134888730 q^{15 / 2}+345764537 q^{8} \\
& +862482876 q^{17 / 2}+2112344321 q^{9}+5061362222 q^{19 / 2}+11921262927 q^{10}+\mathcal{O}\left(q^{21 / 2}\right) . \quad(5.40)
\end{aligned}
$$

One can see the two series match perfectly up to $q^{11 / 2}$ ! 


\begin{tabular}{|c|ccccccccc|}
\hline$i, j$ & 0 & 1 & 2 & 3 & 4 & 5 & 6 & 7 & 8 \\
\hline 0 & 1 & 0 & 31 & 56 & 495 & 1468 & 6269 & 19680 & 64768 \\
1 & 0 & 0 & 0 & 0 & 32 & 58 & 1023 & 3322 & 19078 \\
2 & -1 & -2 & -31 & -60 & -389 & -718 & $-2972+2 \cdot 1$ & $-5226+2 \cdot 2$ & $560-16398+2 \cdot 31+1$ \\
\hline
\end{tabular}

\begin{tabular}{|c|ccc|}
\hline$i, j$ & 9 & 10 & 11 \\
\hline 0 & 187792 & 537021 & 1424526 \\
1 & 69114 & 266799 & 886104 \\
2 & $1912-27570+2 \cdot 60+2$ & $20063-71670+2 \cdot 389+31$ & $83586-115770+2 \cdot 718+60$ \\
\hline
\end{tabular}

Table 15. Series coefficients $c_{i, j}^{\mathrm{SO}(8)}$ for the elliptic genus of two $\mathrm{SO}(8)$ instanton strings.

\begin{tabular}{|c|cccccccccc|}
\hline$i, j$ & 0 & 1 & 2 & 3 & 4 & 5 & 6 & 7 & 8 & 9 \\
\hline 0 & 1 & 0 & 55 & 104 & 1539 & 4966 & 32091 & 119340 & 542109 & 1973088 \\
1 & 0 & 0 & 0 & 0 & 56 & 106 & 3135 & 10900 & 97125 & 405480 \\
2 & 0 & 0 & 0 & 0 & 0 & 0 & 0 & 0 & $1652+1$ & $6040+2$ \\
3 & 1 & 2 & 55 & 108 & 1214 & 2320 & 15802 & 29284 & $143542-1$ & $257800-2$ \\
\hline \multicolumn{1}{c}{$i, j$} & 10 & 11 & 12 & \\
\cline { 2 - 7 } & & 0 & 7460100 & 25288640 & 84766812 & \\
1 & 2210027 & 9075756 & 38900537 & \\
2 & $99611+55$ & $466860+108$ & $3399668+1214$ & \\
3 & $999970-55$ & $1742140-108$ & 5704242 & \\
\end{tabular}

Table 16. Series coefficients $c_{i, j}^{F_{4}}$ for the elliptic genus of two $F_{4}$ instanton strings.

$\boldsymbol{F}_{4}$. Let us denote the two-string elliptic genus with gauge symmetry $F_{4}$ as

$$
\mathbb{E}_{h_{F_{4}}^{(2)}}\left(v, x=1, \tau, m_{i}=0\right)=v^{17} Q_{\tau}^{-17 / 6} \sum_{i, j=0}^{\infty} c_{i, j}^{F_{4}} v^{j}\left(Q_{\tau} v^{-4}\right)^{i} .
$$

Then we have table 16 for the unrefined coefficients $c_{i, j}^{F_{4}}$. The red numbers in the first row agree with the Hilbert series for reduced moduli space of two $F_{4}$ instantons in [42]. By summing over the red numbers in each column, we obtain certain analogy of Schur index of rank two $H_{G}$ theory for $F_{4}$ up to $q^{11 / 2}$. The unrefined version is

$$
\begin{aligned}
1 & +55 q+104 q^{3 / 2}+1595 q^{2}+5072 q^{5 / 2}+35226 q^{3}+130240 q^{7 / 2}+640886 q^{4}+2384608 q^{9 / 2} \\
& +9769738 q^{5}+34831256 q^{11 / 2}+\mathcal{O}\left(q^{6}\right) .
\end{aligned}
$$

This is in complete agreement with Beem-Rastalli's to appear computation from chiral algebra! ${ }^{41}$

\footnotetext{
${ }^{41}$ We thank Beem and Rastelli for providing us their unpublished results on the unrefined Schur index of rank two $F_{4}$ theory.
} 
$\boldsymbol{E}_{\mathbf{6}}$. The $H_{E_{6}}^{(2)}$ theory can be constructed by compactifying $A_{5}(2,0) 6$ d SCFT on a sphere with three $\left\{2^{3}\right\}$ punctures, which is a "bad" theory. One can change one of the punctures to $\left\{2^{2}, 1^{2}\right\}$ to add a decoupled hypermultiplet, i.e. the $\widetilde{H}_{E_{6}}^{(2)}$ theory. The Hall-Littlewood index of this theory was computed in [45]. We expect and indeed checked

$$
\mathcal{I}_{H_{E_{6}}^{(2)}}^{\mathrm{HL}}\left(v, x, m_{E_{6}}\right)=g_{0, E_{6}}^{(2)}\left(v, x, m_{E_{6}}\right) .
$$

The Schur index can be obtained in a similar manner. Following the general formula in [44], we obtain

$$
\begin{aligned}
& \mathcal{I}_{\widetilde{H}_{E_{6}}^{(2)}}^{\text {Schur }}=\hat{\mathcal{N}}_{6} \hat{\mathcal{K}}_{1}\left(a_{1}, a_{2}\right) \hat{\mathcal{K}}_{1}\left(a_{3}, a_{4}\right) \hat{\mathcal{K}}_{2}\left(a_{5}, a_{6}, x\right) \sum_{\lambda} \frac{\chi_{\lambda}\left(v a_{5}, v^{-1} a_{5}, v a_{6}, v^{-1} a_{6}, \frac{x}{a_{5} a_{6}}, \frac{x^{-1}}{a_{5} a_{6}}\right)}{\chi_{\lambda}\left(v^{-5}, v^{-3}, v^{-1}, v^{1}, v^{3}, v^{5}\right)} \\
& \times \chi_{\lambda}\left(v a_{1}, v^{-1} a_{1}, v a_{2}, v^{-1} a_{2}, v \frac{1}{a_{1} a_{2}}, v^{-1} \frac{1}{a_{1} a_{2}}\right) \chi_{\lambda}\left(v a_{3}, v^{-1} a_{3}, v a_{4}, v^{-1} a_{4}, v \frac{1}{a_{3} a_{4}}, v^{-1} \frac{1}{a_{3} a_{4}}\right) .
\end{aligned}
$$

Here $\lambda=\left(\lambda_{1}, \cdots, \lambda_{5}, 0\right)$ and $\left(b_{3} \equiv \frac{1}{b_{1} b_{2}}\right)$

$$
\begin{aligned}
\hat{\mathcal{N}}_{6} & =\left(v^{2} ; v^{2}\right)^{3} \prod_{j=2}^{6}\left(v^{2 j} ; v^{2}\right), \\
\hat{\mathcal{K}}_{1}\left(b_{1}, b_{2}\right) & =\prod_{\ell=1}^{2} \prod_{i, j=1}^{3} \mathrm{PE}\left[\frac{v^{2 \ell} b_{i} / b_{j}}{1-v^{2}}\right], \\
\hat{\mathcal{K}}_{2}\left(b_{1}, b_{2}, x\right) & =\prod_{\ell=1}^{2} \prod_{i, j=1}^{2} \mathrm{PE}\left[\frac{v^{2 \ell} b_{i} / b_{j}}{1-v^{2}}\right] \times \mathrm{PE}\left[\frac{2 v^{2}+v^{2} x^{ \pm 2}+v^{3}\left(b_{1}^{2} b_{2} x^{ \pm 1}\right)^{ \pm 1}+v^{3}\left(b_{1} b_{2}^{2} x^{ \pm 1}\right)^{ \pm 1}}{1-v^{2}}\right] .
\end{aligned}
$$

At last, one needs to replace $v \rightarrow q^{1 / 2}$. We computed the Schur index up to $q^{7}$ :

$$
\begin{aligned}
\mathcal{I}_{H_{E_{6}}^{(2)}}^{\text {Schur }}= & 1+\left(\chi_{3}+78\right) q+78 \chi_{2} q^{3 / 2}+\left(\chi_{5}+79 \chi_{3}+3160\right) q^{2}+\left(78 \chi_{4}+5512 \chi_{2}\right) q^{5 / 2} \\
& +\left(\chi_{7}+79 \chi_{5}+5670 \chi_{3}+87751\right) q^{3}+\left(78 \chi_{6}+5590 \chi_{4}+201292 \chi_{2}\right) q^{7 / 2}+\left(\chi_{9}+79 \chi_{7}\right. \\
& \left.+5671 \chi_{5}+248290 \chi_{3}+1871196\right) q^{4}+\left(78 \chi_{8}+5590 \chi_{6}+250640 \chi_{4}+5048654 \chi_{2}\right) q^{9 / 2} \\
& +\left(\chi_{11}+79 \chi_{9}+5671 \chi_{7}+250400 \chi_{5}+7248975 \chi_{3}+32615793\right) q^{5} \\
& +\left(78 \chi_{10}+5590 \chi_{8}+250718 \chi_{6}+7900243 \chi_{4}+97665932 \chi_{2}\right) q^{11 / 2} \\
& +\left(\chi_{13}+79 \chi_{11}+5671 \chi_{9}+250801 \chi_{7}+7949911 \chi_{5}+157280287 \chi_{3}+483480405\right) q^{6} \\
& +\left(78 \chi_{12}+5590 \chi_{10}+250718 \chi_{8}+7949591 \chi_{6}+18644755 \chi_{4}+1552411211 \chi_{2}\right) q^{13 / 2} \\
& +\left(\chi_{15}+79 \chi_{13}+5671 \chi_{11}+250801 \chi_{9}+7952421 \chi_{7}+193661181 \chi_{5}+2725694921 \chi_{3}\right. \\
& +6263699772) q^{7}+\ldots
\end{aligned}
$$

Note the leading terms up to $q^{7 / 2}$ agree with our general proposal (5.26).

Let us denote the two-string elliptic genus as

$$
\mathbb{E}_{h_{E_{6}}^{(2)}}\left(v, x=1, \tau, m_{i}=0\right)=v^{23} Q_{\tau}^{-23 / 6} \sum_{i, j=0}^{\infty} c_{i, j}^{E_{6}} v^{j}\left(Q_{\tau} v^{-4}\right)^{i} .
$$




\begin{tabular}{|c|cccccccccc|}
\hline$i, j$ & 0 & 1 & 2 & 3 & 4 & 5 & 6 & 7 & 8 & 9 \\
\hline 0 & 1 & 0 & 81 & 156 & 3320 & 11178 & 98440 & 401280 & 2344619 & 9785226 \\
1 & 0 & 0 & 0 & 0 & 82 & 158 & 6723 & 24132 & 296879 & 1335694 \\
2 & 0 & 0 & 0 & 0 & 0 & 0 & 0 & 0 & $3485+1$ & $13112+2$ \\
3 & 0 & 0 & 0 & 0 & 0 & 0 & 0 & 0 & -1 & -2 \\
4 & -1 & -2 & -81 & -160 & -2669 & -5178 & -51445 & -97712 & -681945 & -1266178 \\
\hline
\end{tabular}

\begin{tabular}{|c|cccc|}
\hline$i, j$ & 10 & 11 & 12 & 13 \\
\hline 0 & 45870686 & 182872426 & 746229150 & 2782158570 \\
1 & 9484963 & 44112702 & 236141466 & 1042037420 \\
2 & $301488+81$ & $1497516+160$ & $14405643+2669$ & $75613998+5178$ \\
3 & -81 & -160 & $102090-2669+83$ & $563580-5178+322$ \\
4 & $-6819518+2 \cdot 1$ & $-12372858+2 \cdot 2$ & $-54611704+2 \cdot 81-83$ & $-96850550+2 \cdot 160-322$ \\
\hline
\end{tabular}

\begin{tabular}{|c|cc|}
\hline$i, j$ & 14 & 15 \\
\hline 0 & 10261780870 & 35695088906 \\
1 & 4709271558 & 19202312882 \\
2 & $486421964+51445$ & $2415319754+97712$ \\
3 & $9603627-51445+7039$ & $58071366-97712+24620$ \\
4 & $-365050846+2 \cdot 2669-7039$ & $-633251142+2 \cdot 5178-24620$ \\
\hline
\end{tabular}

Table 17. Series coefficients $c_{i, j}^{E_{6}}$ for the unrefined elliptic genus of two $E_{6}$ instanton strings.

Then we have table 17 for the coefficients $c_{i, j}^{E_{6}}$. Here the red numbers are from the $L_{E_{6}}^{(2)}$ series. Add the red numbers in each column together, we expect to obtain a series that is equal to the Schur index of rank two $H_{E_{6}} 4 \mathrm{~d}$ SCFT. Indeed, we have

$$
\begin{aligned}
L_{E_{6}}^{(2)} & \left(q^{1 / 2}, x=1, m_{E_{6}}=0, q^{2}\right) \\
= & 1+81 q+156 q^{3 / 2}+3402 q^{2}+11336 q^{5 / 2}+105163 q^{3}+425412 q^{7 / 2} \\
& +2644983 q^{4}+11134032 q^{9 / 2}+55655137 q^{5}+228482644 q^{11 / 2}+996878349 q^{6} \\
& +3900373568 q^{13 / 2}+15467078019 q^{7}+57370792908 q^{15 / 2}+\ldots
\end{aligned}
$$

On the other hand, taking $x=1$ in (5.46), the unrefined Schur index is

$$
\begin{gathered}
1+81 q+156 q^{3 / 2}+3402 q^{2}+11336 q^{5 / 2}+105163 q^{3}+425412 q^{7 / 2}+2644983 q^{4}+11134032 q^{9 / 2} \\
+55655137 q^{5}+228482644 q^{11 / 2}+996878349 q^{6}+3900373568 q^{13 / 2}+15467078019 q^{7}+\ldots
\end{gathered}
$$

We can see the two series match perfectly up to $q^{7}$ !

$\boldsymbol{E}_{\mathbf{7}}$. The $H_{E_{7}}^{(2)}$ theory can be constructed by compactifying $A_{7}(2,0) 6 \mathrm{~d}$ SCFT on a sphere with one $\left\{4^{2}\right\}$ puncture and two $\left\{2^{4}\right\}$ punctures, which is a "bad" theory. One can change one of the $\left\{2^{4}\right\}$ punctures to $\left\{2^{3}, 1^{2}\right\}$ to add a decoupled hypermultiplet, i.e. the $\widetilde{H}_{E_{7}}^{(2)}$ theory. The Hall-Littlewood index of this theory was computed in [45]. We find it agrees with our computation for $g_{0, E_{7}}^{(2)}\left(\tau, x, m_{E_{7}}\right)$. The Schur index can be obtained in a similar 


\begin{tabular}{|c|cccccccccc|}
\hline$i, j$ & 0 & 1 & 2 & 3 & 4 & 5 & 6 & 7 & 8 & 9 \\
\hline 0 & 1 & 0 & 136 & 266 & 9315 & 32830 & 449050 & 2026080 & 17179899 & 84195608 \\
1 & 0 & 0 & 0 & 0 & 137 & 268 & 18768 & 69544 & 1349005 & 6575250 \\
2 & 0 & 0 & 0 & 0 & 0 & 0 & 0 & 0 & $9590+1$ & $36982+2$ \\
\hline
\end{tabular}

Table 18. Series coefficients $c_{i, j}^{E_{7}}$ for the unrefined elliptic genus of two $E_{7}$ instanton.

manner. Following the general formula in [44], we obtain

$$
\begin{aligned}
\mathcal{I}_{\widetilde{H}_{E_{7}}^{(2)}}^{\text {Schur }}= & \hat{\mathcal{N}}_{8} \hat{\mathcal{K}}_{1}\left(a_{1}, a_{2}, a_{3}\right) \hat{\mathcal{K}}_{2}\left(a_{4}, a_{5}, a_{6}, x\right) \hat{\mathcal{K}}_{3}\left(a_{7}\right) \\
& \times \sum_{\lambda} \frac{\chi_{\lambda}\left(v^{3} a_{7}, v^{-3} a_{7}, v a_{7}, v^{-1} a_{7}, v^{3} a_{7}^{-1}, v^{-3} a_{7}^{-1}, v a_{7}^{-1}, v^{-1} a_{7}^{-1}\right)}{\chi_{\lambda}\left(v^{-7}, v^{-5}, v^{-3}, v^{-1}, v, v^{3}, v^{5}, v^{7}\right)} \\
& \times \chi_{\lambda}\left(v a_{1}, v^{-1} a_{1}, v a_{2}, v^{-1} a_{2}, v a_{3}, v^{-1} a_{3}, v \frac{1}{a_{1} a_{2} a_{3}}, v^{-1} \frac{1}{a_{1} a_{2} a_{3}}\right) \\
& \times \chi_{\lambda}\left(v a_{4}, v^{-1} a_{4}, v a_{5}, v^{-1} a_{5}, v a_{6}, v^{-1} a_{6}, \frac{x}{a_{4} a_{5} a_{6}}, \frac{x^{-1}}{a_{4} a_{5} a_{6}}\right) .
\end{aligned}
$$

Here

$$
\begin{aligned}
\hat{\mathcal{K}}_{3}(b) & =\mathrm{PE}\left[\frac{2\left(v^{2}+v^{4}+v^{6}+v^{8}\right)}{1-v^{2}}\right] \prod_{\ell=1}^{4} \mathrm{PE}\left[\frac{v^{2 \ell} b^{ \pm 2}}{1-v^{2}}\right] \\
\hat{\mathcal{K}}_{2}\left(b_{1}, b_{2}, b_{3}, x\right) & =\mathrm{PE}\left[\frac{2 v^{2}+v^{2} x^{ \pm 2}}{1-v^{2}}\right]\left[\prod_{\ell=1}^{2} \prod_{i, j=1}^{3} \mathrm{PE}\left[\frac{v^{2 \ell} b_{i} / b_{j}}{1-v^{2}}\right]\right], \prod_{i=1}^{3}\left[\frac{v^{3}\left(x^{-1} b_{i} / b_{4}\right)^{ \pm 1}+v^{3}\left(x b_{i} / b_{4}\right)^{ \pm 1}}{1-v^{2}}\right], \\
\hat{\mathcal{K}}_{1}\left(b_{1}, b_{2}, b_{3}\right) & =\prod_{\ell=1}^{2} \prod_{i, j=1}^{4} \mathrm{PE}\left[\frac{v^{2 \ell} b_{i} / b_{j}}{1-v^{2}}\right] .
\end{aligned}
$$

At last, one needs to replace $v \rightarrow q^{1 / 2}$. We computed the Schur index up to $q^{2}$ order.

After decoupling the free hypermultiplet, the Schur index of $H_{E_{7}}^{(2)}$ theory is given by

$\mathcal{I}_{H_{E_{7}}^{(2)}}^{\mathcal{S} \text { Schur }}=\mathcal{I}_{\widetilde{H}_{E_{7}}^{(2)}}^{\text {Schur }} / \mathcal{I}_{h . m .}^{\text {Schur }}=1+\left(\chi_{3}+\mathbf{1 3 3}\right) q+\mathbf{1 3 3} \chi_{2} q^{3 / 2}+\left(\chi_{5}+(\mathbf{1 3 3}+1) \chi_{3}+\operatorname{Sym}^{2} \mathbf{1 3 3}+\mathbf{1 3 3}+1\right) q^{2}+\ldots$

Let us denote the two-string elliptic genus as

$$
\mathbb{E}_{h_{E_{7}}^{(2)}}\left(v, x=1, \tau, m_{i}=0\right)=v^{35} Q_{\tau}^{-35 / 6} \sum_{i, j=0}^{\infty} c_{i, j}^{E_{7}} v^{j}\left(Q_{\tau} v^{-4}\right)^{i} .
$$

Then we have table 18 for the coefficients $c_{i, j}^{E_{7}}$. Here the red numbers are from the $L_{E_{7}}^{(2)}$ series. Add the red numbers in each column together, we expect to obtain a series that is equal to the Schur index of rank two $H_{E_{7}} 4 \mathrm{~d}$ SCFT. Thus, we predict the unrefined Schur index as

$$
\begin{aligned}
1 & +136 q+266 q^{3 / 2}+9452 q^{2}+33098 q^{5 / 2}+467818 q^{3}+2095624 q^{7 / 2}+18538494 q^{4} \\
& +90807840 q^{9 / 2}+\mathcal{O}\left(q^{5}\right)
\end{aligned}
$$


Indeed, taking $x=1$ in (5.52), the unrefined Schur index is given by

$$
1+136 q+266 q^{3 / 2}+9452 q^{2}+\mathcal{O}\left(q^{5 / 2}\right) .
$$

We can see the two series match perfectly!

$\boldsymbol{E}_{\mathbf{8}}$. The $H_{E_{8}}^{(2)}$ theory can be constructed by compactifying $A_{11}(2,0) 6 \mathrm{~d}$ SCFT on a sphere with three $\left\{6^{2}\right\},\left\{4^{3}\right\}$ and $\left\{2^{6}\right\}$, which is a "bad" theory. One can change the $\left\{2^{6}\right\}$ puncture to $\left\{2^{5}, 1^{2}\right\}$ to add a decoupled free hypermultiplet, i.e. the $\widetilde{H}_{E_{8}}^{(2)}$ theory. Following the general formula in [44], we obtain its Schur index as

$$
\begin{aligned}
\mathcal{I}_{\widetilde{H}_{E_{8}}^{(2)}}^{\text {Schur }}= & \hat{\mathcal{N}}_{12} \hat{\mathcal{K}}_{1}\left(a_{1}, a_{2}, a_{3}, a_{4}, a_{5}, x\right) \hat{\mathcal{K}}_{2}\left(a_{6}, a_{7}\right) \hat{\mathcal{K}}_{3}\left(a_{8}\right) \sum_{\lambda} \frac{\chi_{\lambda}\left(v a_{1}, v^{-1} a_{1}, \ldots, v a_{5}, v^{-1} a_{5}, \frac{x}{a_{1} \cdots a_{5}}, \frac{x^{-1}}{a_{1} \cdots a_{5}}\right)}{\chi_{\lambda}\left(v^{-11}, v^{-9}, \ldots, v^{9}, v^{11}\right)} \\
& \times \chi_{\lambda}\left(v^{3} a_{6}, v^{-3} a_{6}, v a_{6}, v^{-1} a_{6}, v^{3} a_{7}, v^{-3} a_{7}, v a_{7}, v^{-1} a_{7}, v^{3} \frac{1}{a_{6} a_{7}}, v^{-3} \frac{1}{a_{6} a_{7}}, v \frac{1}{a_{6} a_{7}}, v^{-1} \frac{1}{a_{6} a_{7}}\right) \\
& \times \chi_{\lambda}\left(v^{-5} a_{8}, v^{-3} a_{8}, \ldots, v^{3} a_{8}, v^{5} a_{8}, v^{-5} a_{8}^{-1}, v^{-3} a_{8}^{-1}, \ldots, v^{3} a_{8}^{-1}, v^{5} a_{8}^{-1}\right)
\end{aligned}
$$

Here $\lambda=\left(\lambda_{1}, \cdots, \lambda_{11}, 0\right)$ and

$$
\begin{aligned}
\hat{\mathcal{K}}_{3}(b) & =\prod_{\ell=1}^{6} \mathrm{PE}\left[\frac{2 v^{2 \ell}+v^{2 \ell} b^{ \pm 2}}{1-v^{2}}\right], \\
\hat{\mathcal{K}}_{2}\left(b_{1}, b_{2}\right)= & \prod_{\ell=1}^{4} \prod_{i, j=1}^{3} \mathrm{PE}\left[\frac{v^{2 \ell} b_{i} / b_{j}}{1-v^{2}}\right], \\
\hat{\mathcal{K}}_{1}\left(c_{1}, c_{2}, c_{3}, c_{4}, c_{5}, x\right)= & \operatorname{PE}\left[\frac{2 v^{2}+v^{2} x^{ \pm 2}}{1-v^{2}}\right]\left[\prod_{\ell=1}^{2} \prod_{i, j=1}^{5} \mathrm{PE}\left[\frac{v^{2 \ell} c_{i} / c_{j}}{1-v^{2}}\right]\right] \\
& \times \prod_{i=1}^{5} \mathrm{PE}\left[\frac{v^{3}\left(x^{-1} c_{i} / c_{6}\right)^{ \pm 1}+v^{3}\left(x c_{i} / c_{6}\right)^{ \pm 1}}{1-v^{2}}\right],
\end{aligned}
$$

where $b_{3} \equiv \frac{1}{b_{1} b_{2}}$ and $c_{6} \equiv \frac{1}{c_{1} c_{2} c_{3} c_{4} c_{5}}$. At last, one needs to replace $v \rightarrow q^{1 / 2}$. As the leading terms up to $q^{3 / 2}$ are contributed from rank one theory, the Schur index is given by

$$
\mathcal{I}_{\widetilde{H}_{E_{8}}^{(2)}}^{\text {Schur }}=1+\chi_{2} q^{1 / 2}+\left(2 \chi_{3}+\mathbf{2 4 8}\right) q+\left(2 \chi_{4}+2(\mathbf{2 4 8}+1) \chi_{2}\right) q^{3 / 2}+\ldots
$$

After decoupling the free hypermultiplet, the Schur index of $H_{E_{8}}^{(2)}$ theory is

$$
\mathcal{I}_{H_{E_{8}}^{(2)}}^{\text {Schur }}=\mathcal{I}_{\widetilde{H}_{E_{7}}^{(2)}}^{\text {Schur }} / \mathcal{I}_{h . m .}^{\text {Schur }}=1+\left(\chi_{3}+\mathbf{2 4 8}\right) q+\mathbf{2 4 8} \chi_{2} q^{3 / 2}+\ldots
$$

Let us denote the two-string elliptic genus as

$$
\mathbb{E}_{h_{E_{8}}^{(2)}}\left(v, x=1, \tau, m_{i}=0\right)=v^{59} Q_{\tau}^{-59 / 6} \sum_{i, j=0}^{\infty} c_{i, j}^{E_{8}} v^{j}\left(Q_{\tau} v^{-4}\right)^{i} .
$$

Then we have table 19 for the coefficients $c_{i, j}^{E_{8}}$. Here the red numbers are from the $L_{E_{8}}^{(2)}$ series. Add the red numbers in each column together, we expect to obtain a series that is 


\begin{tabular}{|c|cccccccc|}
\hline$i, j$ & 0 & 1 & 2 & 3 & 4 & 5 & 6 & 7 \\
\hline 0 & 1 & 0 & 251 & 496 & 31625 & 116248 & 2747875 & 13624000 \\
1 & 0 & 0 & 0 & 0 & 252 & 498 & 63503 & 241742 \\
\hline
\end{tabular}

Table 19. Series coefficients $c_{i, j}^{E_{8}}$ for the unrefined elliptic genus of two $E_{8}$ instanton strings.

equal to the Schur index of rank two $H_{E_{8}} 4 \mathrm{~d}$ SCFT. Thus, we predict from the general formula (5.26) for the unrefined Schur index as

$$
1+251 q+496 q^{3 / 2}+31877 q^{2}+116746 q^{5 / 2}+2811378 q^{3}+13865742 q^{7 / 2}+\mathcal{O}\left(q^{4}\right)
$$

Indeed, taking $x=1$ in (5.59), the unrefined Schur index is given by

$$
1+251 q+496 q^{3 / 2}+\mathcal{O}\left(q^{2}\right) .
$$

Indeed, the two series match perfectly!

\subsection{Rank three and higher}

We expect the Del Zotto-Lockhart's conjecture can be generalized to rank three and higher. From the universal leading expansion for three-string elliptic genus (4.11) and (4.12), we are able to predict the Schur index of rank three $H_{G}$ SCFT up to order $q^{3}$ :

$$
\begin{aligned}
\mathcal{I}_{H_{G}^{(3)}}^{\text {Schur }}= & 1+\left(\chi_{3}+\chi_{\theta}\right) q+\left(\chi_{4}+\chi_{\theta} \chi_{2}\right) q^{3 / 2}+\left(\chi_{5}+\left(\chi_{\theta}+1\right) \chi_{3}+\chi_{\mathrm{Sym}^{2} \theta}+\chi_{\theta}+2\right) q^{2} \\
& +\left(\chi_{6}+\left(2 \chi_{\theta}+2\right) \chi_{4}+2 \chi_{\mathrm{Sym}^{2} \theta}+\chi_{\theta}+1\right) q^{5 / 2} \\
& +\left(2 \chi_{7}+\left(3 \chi_{\theta}+1\right) \chi_{5}+\left(\chi_{2 \theta}+3 \chi_{\mathrm{Sym}^{2} \theta}+3 \chi_{\theta}+5\right) \chi_{3}+\chi_{\mathrm{Sym}^{3} \theta}+3 \chi_{\mathrm{Sym}^{2} \theta}+\chi_{\theta}+2\right) q^{3} \\
& +\mathcal{O}\left(q^{7 / 2}\right) .
\end{aligned}
$$

This is actually because (4.11) and (4.12) are also the definition of leading and subleading $Q_{\tau}$ order of $L_{G}^{(3)}$ functions. Besides, we observe in the coefficient matrix of reduced three string elliptic genus, other than the $L_{G}^{(3)}$ function that appears as expected, the blue series also appears as in the rank two. The difference is that here the blue series is generated from the leading $Q_{\tau}$ order of two string elliptic genus!

$$
M_{G}^{(3), \text { blue }}(v, x)=\frac{1}{(1-v x)(1-v / x)} g_{0, G}^{(2)}(v, x) .
$$

Note $g_{0, G}^{(2)}(v, x)$ is also the leading $Q_{\tau}$ order of $L_{G}^{(2)}$. In the following, we show the relation between reduced elliptic genus of three strings and the Schur index of $H_{G}^{(3)}$ theories for each $G$.

SU(3). The formula for the elliptic genus of three $\mathrm{SU}(3)$ string has been written down via Jeffrey-Kirwan residues in [34], using which we computed $\mathbb{E}_{h_{A_{2}}^{(3)}}$ up to $Q_{\tau}^{6}$ order. Denote

$$
\mathbb{E}_{h_{A_{2}}^{(3)}}\left(v, x, \tau, m_{i}=0\right)=v^{8} Q_{\tau}^{-4 / 3} \sum_{i, j=0}^{\infty} c_{i, j}^{\mathrm{SU}(3)}(x) v^{j}\left(Q_{\tau} v^{-4}\right)^{i}
$$




\begin{tabular}{|c|cccccccc|}
\hline$i, j$ & 0 & 1 & 2 & 3 & 4 & 5 & 6 & 7 \\
\hline 0 & 1 & 0 & 11 & 20 & 90 & 218 & 698 & 1618 \\
1 & 1 & 2 & 14 & 22 & $135+12$ & $370+22$ & $960+171$ & $2250+502$ \\
\hline
\end{tabular}

Table 20. Coefficients $c_{i, j}^{\mathrm{SU}(3)}$ for the unrefined elliptic genus of three $\mathrm{SU}(3)$ instanton strings.

Then the unrefined $L_{G}^{(3)}$ function is shown red in the coefficient matrix of $\mathbb{E}_{h_{A_{2}}^{(3)}}$ in table 20. Note the red numbers are in agreement with our universal expansion (4.11) and (4.12), while the blue numbers are in agreement with our proposal (5.64).

The construction for rank three $H_{A_{2}}$ theory from $6 \mathrm{~d}$ involves certain irregular punctures as the rank two case. We are not aware how to compute its indices directly. We write down our prediction for the Schur index of rank three $H_{A_{2}}$ theory here:

$$
\begin{aligned}
\mathcal{I}_{H_{A_{2}}^{(3)}}^{\text {Schur }}= & 1+\left(\chi_{3}+8\right) q+\left(\chi_{4}+8 \chi_{2}\right) q^{3 / 2}+\left(\chi_{5}+17 \chi_{3}+46\right) q^{2}+\left(\chi_{6}+18 \chi_{4}+81 \chi_{2}\right) q^{5 / 2} \\
& +\left(2 \chi_{7}+25 \chi_{5}+164 \chi_{3}+248\right) q^{3}+\left(\chi_{8}+27 \chi_{6}+209 \chi_{4}+557 \chi_{2}\right) q^{7 / 2}+\mathcal{O}\left(q^{4}\right) .
\end{aligned}
$$

The unrefined limit is

$$
\mathcal{I}_{H_{A_{2}}^{(3)}}^{\text {Schur }}(x=1)=1+11 q+20 q^{3 / 2}+102 q^{2}+240 q^{5 / 2}+869 q^{3}+2120 q^{7 / 2}+\mathcal{O}\left(q^{4}\right) .
$$

$\mathbf{S O}(8)$. We can use class $\mathcal{S}$ theory technique to compute the HL and Schur index of rank three $H_{D_{4}} 4 \mathrm{~d}$ SCFT. The $H_{D_{4}}^{(3)}$ theory can be constructed by compactifying $A_{5}(2,0) 6 \mathrm{~d}$ SCFT on a sphere with four $\left\{3^{2}\right\}$ punctures, which is a "bad" theory. We need instead to consider $\widetilde{H}_{D_{4}}^{(3)}$ theory obtained from three $\left\{3^{2}\right\}$ punctures and one $\{3,2,1\}$ puncture. We compute the Schur index as

$$
\begin{aligned}
\mathcal{I}_{\widetilde{H}_{D_{4}}^{(3)}}^{\text {Schur }}\left(c_{1}, c_{2}, c_{3} ; x, b\right) \\
=\hat{\mathcal{N}}_{6}^{\prime} \hat{\mathcal{K}}_{1}(c) \hat{\mathcal{K}}_{1}(d) \hat{\mathcal{K}}_{1}(e) \hat{\mathcal{K}}_{2}(x, b) \sum_{\lambda} \frac{\chi_{\lambda}\left(v^{2} b, b, v^{-2} b, v b^{-1} x, v^{-1} b^{-1} x, b^{-1} x^{-2}\right)}{\chi_{\lambda}^{2}\left(v^{-5}, v^{-3}, v^{-1}, v, v^{3}, v^{5}\right)} \\
\quad \times \prod_{i=1,2,3} \chi_{\lambda}\left(v^{2} c_{i}, c_{i}, v^{-2} c_{i}, v^{2} c_{i}^{-1}, c_{i}^{-1}, v^{-2} c_{i}^{-1}\right),
\end{aligned}
$$

with $\left(b_{1}=b, b_{2}=1 / b\right)$

$$
\begin{aligned}
\hat{\mathcal{N}}_{6}^{\prime} & =\left(v^{2} ; v^{2}\right)^{4} \prod_{j=2}^{6}\left(v^{2 j} ; v^{2}\right)^{2} \\
\hat{\mathcal{K}}_{1}(b) & =\operatorname{PE}\left[\frac{\left(v^{2}+v^{4}+v^{6}\right)\left(b^{2}+b^{-2}+2\right)}{1-v^{2}}\right], \\
\hat{\mathcal{K}}_{2}(a, b) & =\operatorname{PE}\left[\frac{3 v^{2}+2 v^{4}+v^{6}+\left(v^{3}+v^{5}\right)\left(b^{2} a^{-1}\right)^{ \pm 1}+v^{3} a^{ \pm 3}+v^{4}\left(b^{2} a^{2}\right)^{ \pm 1}}{1-v^{2}}\right] .
\end{aligned}
$$




\begin{tabular}{|c|ccccccc|}
\hline$i, j$ & 0 & 1 & 2 & 3 & 4 & 5 & 6 \\
\hline 0 & 1 & 0 & 31 & 60 & 580 & 1858 & 9457 \\
1 & 0 & 0 & 0 & 0 & 32 & 62 & 1111 \\
\hline
\end{tabular}

Table 21. Expected coefficients $c_{i, j}^{\mathrm{SO}(8)}$ for the unrefined elliptic genus of three $\mathrm{SO}(8)$ instanton strings.

From the above formula, we compute the Schur index up to $q^{11 / 2}$. After decoupling the free hypermultiplet, we obtain

$$
\begin{aligned}
\mathcal{I}_{H_{D_{4}}^{(3)}}^{\text {Schur }}= & \mathcal{I}_{\widetilde{H}_{D_{4}}^{(S) h u r}}^{\text {Sch.m. }}=1+\left(\chi_{3}^{\text {Schur }}+28\right) q+\left(\chi_{4}+28 \chi_{2}\right) q^{3 / 2}+\left(\chi_{5}+57 \chi_{3}+436\right) q^{2} \\
& +\left(\chi_{6}+58 \chi_{4}+841 \chi_{2}\right) q^{5 / 2}+\left(2 \chi_{7}+85 \chi_{5}+1607 \chi_{3}+5308\right) q^{3} \\
& +\left(\chi_{8}+87 \chi_{6}+2042 \chi_{4}+14135 \chi_{2}\right) q^{7 / 2}+\left(2 \chi_{9}+115 \chi_{7}+2806 \chi_{5}+29042 \chi_{3}+55871\right) q^{4} \\
& +\left(2 \chi_{10}+115 \chi_{8}+3242 \chi_{6}+43166 \chi_{4}+177896 \chi_{2}\right) q^{9 / 2} \\
& +\left(2 \chi_{11}+144 \chi_{9}+4008 \chi_{7}+60673 \chi_{5}+392233 \chi_{3}+527217\right) q^{5} \\
& +\left(2 \chi_{12}+145 \chi_{10}+4441 \chi_{8}+75128 \chi_{6}+649112 \chi_{4}+1857119 \chi_{2}\right) q^{11 / 2}+\mathcal{O}\left(q^{6}\right) .
\end{aligned}
$$

The unrefined limit is

$$
\begin{aligned}
\mathcal{I}_{H_{D_{4}}^{(3)}}^{\text {Schur }}(x=1)= & 1+31 q+60 q^{3 / 2}+612 q^{2}+1920 q^{5 / 2}+10568 q^{3}+36968 q^{7 / 2}+157850 q^{4} \\
& +548848 q^{9 / 2}+2036655 q^{5}+6798456 q^{11 / 2}+\mathcal{O}\left(q^{6}\right) .
\end{aligned}
$$

Let us denote the reduced three-string elliptic genus as

$$
\mathbb{E}_{h_{D_{4}}^{(3)}}\left(v, x, \tau, m_{i}=0\right)=v^{17} Q_{\tau}^{-17 / 6} \sum_{i, j=0}^{\infty} c_{i, j}^{\mathrm{SO}(8)} v^{j}\left(Q_{\tau} v^{-4}\right)^{i} .
$$

Then from (4.11) and (4.12), we expect to have table 21 for the unrefined coefficients $c_{i, j}^{\mathrm{SO}(8)}$. Here the red numbers are from the $L_{D_{4}}^{(3)}$ series. Add the red numbers in each column together, we expect to obtain a series that is equal to the Schur index of rank three $H_{D_{4}} 4 \mathrm{~d}$ SCFT. Indeed, we have

$$
L_{D_{4}}^{(3)}\left(q^{1 / 2}, x=1, Q_{m}=1, q^{2}\right)=1+31 q+60 q^{3 / 2}+612 q^{2}+1920 q^{5 / 2}+10568 q^{3}+\ldots
$$

One can see the two series match perfectly up to $q^{3}$ !

$\boldsymbol{E}_{\mathbf{6}}$. The formula to compute the Hall-Littlewood index of rank three $H_{E_{6}}$ SCFT has been written down in [45]. Similarly, we compute the Schur index as

$$
\begin{aligned}
\mathcal{I}_{\widetilde{H}_{E_{6}}^{(3)}}^{\text {Schur }}= & \hat{\mathcal{N}}_{9} \hat{\mathcal{K}}_{1}\left(a_{1}, a_{2}\right) \hat{\mathcal{K}}_{1}\left(a_{3}, a_{4}\right) \hat{\mathcal{K}}_{2}\left(a_{5}, a_{6}, x\right) \times \\
& \sum_{\lambda} \frac{\chi_{\lambda}\left(v^{2} a_{5}, v^{-2} a_{5}, a_{5}, v^{2} a_{6}, v^{-2} a_{6}, a_{6}, v \frac{x}{a_{5} a_{6}}, v^{-1} \frac{x}{a_{5} a_{6}}, \frac{x^{-2}}{a_{5} a_{6}}\right)}{\chi_{\lambda}\left(v^{-8}, v^{-6}, v^{-4}, v^{-2}, 1, v^{2}, v^{4}, v^{6}, v^{8}\right)} \times \\
& \times \chi_{\lambda}\left(v^{2} a_{1}, v^{-2} a_{1}, a_{1}, v^{2} a_{2}, v^{-2} a_{2}, a_{2}, v^{2} \frac{1}{a_{1} a_{2}}, v^{-2} \frac{1}{a_{1} a_{2}}, \frac{1}{a_{1} a_{2}}\right) \\
& \times \chi_{\lambda}\left(v^{2} a_{3}, v^{-2} a_{3}, a_{3}, v^{2} a_{4}, v^{-2} a_{4}, a_{4}, v^{2} \frac{1}{a_{3} a_{4}}, v^{-2} \frac{1}{a_{3} a_{4}}, \frac{1}{a_{3} a_{4}}\right) .
\end{aligned}
$$




\begin{tabular}{|c|ccccccc|}
\hline$i, j$ & 0 & 1 & 2 & 3 & 4 & 5 & 6 \\
\hline 0 & 1 & 0 & 81 & 160 & 3555 & 12958 & 121447 \\
1 & 0 & 0 & 0 & 0 & 82 & 162 & 6961 \\
\hline
\end{tabular}

Table 22. Coefficients $c_{i, j}^{E_{6}}$ for the unrefined elliptic genus of three $E_{6}$ instanton strings.

with

$$
\begin{aligned}
& \hat{\mathcal{K}}_{1}\left(b_{1}, b_{2}\right)=\mathrm{PE} {\left[\sum_{i, j=1}^{3} \frac{\left(v^{2}+v^{4}+v^{6}\right) b_{i} / b_{j}}{1-v^{2}}\right], } \\
& \hat{\mathcal{K}}_{2}\left(b_{1}, b_{2}, x\right)=\mathrm{PE} {\left[\frac { 1 } { 1 - v ^ { 2 } } \left(\left(v^{2}+v^{4}+v^{6}\right)\left(\sum_{i, j=1}^{2} b_{i} / b_{j}\right)+2 v^{2}+v^{4}+v^{3} x^{ \pm 3}\right.\right.} \\
&\left.\left.+\left(b_{1}+b_{2}\right)\left(\left(v^{3}+v^{5}\right)\left(b_{3} x\right)^{ \pm 1}+v^{4}\left(b_{3} x^{-2}\right)^{ \pm 1}\right)\right)\right]
\end{aligned}
$$

where $b_{1} b_{2} b_{3}=1$. From the above formula, we computed the Schur index up to $q^{2}$. After decoupling the free hypermultiplet, we obtain

$$
\begin{aligned}
\mathcal{I}_{H_{E_{6}}^{(3)}}^{\text {Schur }}=\mathcal{I}_{\widetilde{H}_{E_{6}}^{(3)}}^{\text {Schur }} / \mathcal{I}_{h . m .}^{\text {Schur }}= & 1+\left(\chi_{3}+78\right) q+\left(\chi_{4}+78 \chi_{2}\right) q^{3 / 2}+\left(\chi_{5}+157 \chi_{3}+3161\right) q^{2} \\
& +\left(\chi_{6}+158 \chi_{4}+6241 \chi_{2}\right) q^{5 / 2}+\left(2 \chi_{7}+235 \chi_{5}+11912 \chi_{3}+91483\right) q^{3} \\
& +\left(\chi_{8}+237 \chi_{6}+15072 \chi_{4}+260821 \chi_{2}\right) q^{7 / 2}+\mathcal{O}\left(q^{4}\right)
\end{aligned}
$$

The unrefined limit is

$$
\mathcal{I}_{H_{E_{6}}^{(3)}}^{\operatorname{Schur}}(x=1)=1+81 q+160 q^{3 / 2}+3637 q^{2}+13120 q^{5 / 2}+128408 q^{3}+583360 q^{7 / 2}+\mathcal{O}\left(q^{4}\right)
$$

On the other hand, the universal leading expansion (4.11) and (4.12) indicate the following table 22 for the coefficients of reduced three-string elliptic genus for $E_{6}$. By adding the red numbers in each column together, one can indeed obtain the same unrefined Schur series as (5.75) up to $q^{3}$.

$\boldsymbol{F}_{\mathbf{4}}, \boldsymbol{E}_{\mathbf{7}}, \boldsymbol{E}_{\mathbf{8}}$. The Schur indices with generic SU $(2)_{x}$ fugacity for rank three $H_{G}$ theories can be predicted from (5.63) up to $q^{3}$ order. Let us just mark the unrefined series here:

$$
\begin{aligned}
& \mathcal{I}_{H_{F_{4}}^{(3)}}^{\mathrm{Schur}}=1+55 q+108 q^{3 / 2}+1752 q^{2}+6048 q^{5 / 2}+45835 q^{3}+\mathcal{O}\left(q^{7 / 2}\right), \\
& \mathcal{I}_{H_{E_{7}}^{(3)}}^{\mathrm{Schur}}=1+136 q+270 q^{3 / 2}+9852 q^{2}+36990 q^{5 / 2}+533401 q^{3}+\mathcal{O}\left(q^{7 / 2}\right), \\
& \mathcal{I}_{H_{E_{8}}^{(3)}}^{\mathrm{Schur}}=1+251 q+500 q^{3 / 2}+32622 q^{2}+126000 q^{5 / 2}+3030748 q^{3}+\mathcal{O}\left(q^{7 / 2}\right) .
\end{aligned}
$$

Note for $F_{4}$, we always mean the analogy for $H_{G}$ theories. 
In summary, we arrive at the final conjecture for arbitrary rank:

Conjecture. There exists an infinite series of functions $L_{G}^{(n)}\left(v, x, m_{G}, Q_{\tau}\right)=\sum_{i, j=0}^{\infty} b_{i, j}^{G, n} Q_{\tau}^{i} v^{j}$, $n=1,2, \ldots$ such that

1. $b_{i, j}^{G, n}$ can be written as the sum of products between the characters of irreducible representations of $\mathrm{SU}(2)_{x}$ and the characters of irreducible representations of $G$ with integeral coefficients.

2. $L_{G}^{(n)}\left(v, x, m_{G}, 0\right)$ is the Hilbert series of the reduced moduli space of $n G$-instantons, i.e. the Hall-Littlewood index of the $H_{G}^{(n)}$ theory.

3. $L_{G}^{(n)}\left(q^{1 / 2}, x, m_{G}, q^{2}\right)$ is the Schur index of the $H_{G}^{(n)}$ theory.

4. The $n$-string elliptic genus $\mathbb{E}_{h_{G}^{(n)}}\left(v, x, m_{G}, Q_{\tau}\right)$ can be generated from the first $n L_{G}$ functions, i.e. $L_{G}^{(r)}\left(v, x, m_{G}, Q_{\tau}\right), r=1,2, \ldots, n$.

\section{Conclusion and outlook}

In this paper we study the elliptic blowup equations for minimal $6 \mathrm{~d}(1,0)$ SCFTs with all six possible gauge groups $G=\mathrm{SU}(3), \mathrm{SO}(8), F_{4}, E_{6,7,8}$. The study is twofold, topological string partition function on elliptic non-compact Calabi-Yau and elliptic genera for $6 \mathrm{~d}(1,0)$ SCFTs. From the viewpoint of Calabi-Yau, we use the geometric construction in [8] and the generalized blowup equations in [15] to solve the refined BPS invariants to high base degrees, which in turn serve as numerous nontrivial checks for the blowup equations, both unity and vanishing ones. From the viewpoint of $6 \mathrm{~d}$ SCFTs, we use the de-affinisation procedure to derive some elegant functional equations for the elliptic genera, from which we obtain an exact and universal recursion formula for the elliptic genera of arbitrary number of strings and arbitrary gauge group. In particular, we explicitly compute the one and two-string elliptic genera for all $G$, which recover all previous partial results from refined topological string, modular bootstrap, Hilbert series, $2 \mathrm{~d}$ quiver gauge theories and the $\beta$-twisted partition function of $\mathcal{N}=2$ superconformal $H_{G}$ theories. We also prove the modularity of the elliptic blowup equations which is a strong support that they hold for arbitrary number of strings.

The elliptic genera we solve from blowup equations could be useful in many aspects. For example, they would help to identify the $2 \mathrm{~d}$ quiver description of the $6 \mathrm{~d}$ minimal SCFT with exceptional gauge symmetry, see some attempts for $G=E_{7}$ in [87]. They also serve as the calibration to determine modular ansatz for higher-string elliptic genus and the web of topological vertex for the associated non-toric Calabi-Yau threefolds [40]. The elliptic genera of $2 \mathrm{~d}(0,4)$ SCFTs we studied also play a role in the context of certain compact elliptic Calabi-Yau threefolds [88, 89]. For example, the $\mathrm{SO}(8)$ and $E_{6}$ minimal SCFT serve as the constituents of the $2 \mathrm{~d}$ quiver gauge theories associated to the $T^{6} / \mathbb{Z}_{2} \times \mathbb{Z}_{2}$ and $T^{6} / \mathbb{Z}_{3} \times \mathbb{Z}_{3}$ geometries respectively, and their elliptic genera are useful to compute the degeneracies of $5 \mathrm{~d}$ spinning BPS black holes in the dual gravity picture, as suggested in [89]. We hope our exact formulas of the elliptic genera for exceptional minimal SCFTs would contribute to this subject. 
It is also interesting to investigate the K-theoretic blowup equations for all possible 5d SYM theories. The K-theoretic blowup equations are quite different from the elliptic ones in that they exist for all simple Lie groups. Part of the unity K-theoretic blowup equations were already conjectured by Nakajima-Yoshioka [28] and explicitly checked by Keller-Song [30]. One may suggest to use dimensional reduction i.e. $Q_{\tau} \rightarrow 0$ to obtain the K-theoretic blowup equations for the six gauge groups $G$. However, intriguingly we find that the $5 \mathrm{~d}$ reduction of elliptic blowup equations does not produce all non-equivalent $\mathrm{K}$-theoretic blowup equations for these six gauge groups. We leave these issues and the complete set of K-theoretic blowup equations for all simple Lie group to future work.

The elliptic blowup equations for $6 \mathrm{~d}(1,0)$ minimal SCFTs allow us to further study more complicated examples. Some immediate models are the non-Higgsable clusters with matters which we will investigate in a subsequent paper [51]. There are four of them: one belongs to minimal $(1,0)$ SCFT with $\mathfrak{n}=7$, and the other three have more than one dimensional tensor branch. See some primary results on the elliptic genera for such theories in $[8,38,87]$. One can also use blowup equations to study elliptic Calabi-Yau with multisections. See some discussion on such geometries in [90, 91]. Our final goal is to find an exact, explicit and universal formula for the elliptic genera of all 6d $(1,0)$ SCFTs in the atomic classification [6], see also a good review on the classification in [92]. It is known there are two approaches to classifying 6d $(1,0)$ SCFTs: top down and bottom up. We expect blowup equations make sense in both settings. In the top down approach, given the explicit description of a elliptic non-compact Calabi-Yau, i.e. base and elliptic fibration, one should be able to use the generalized blowup equations in [15] to solve the partition function of refined topological strings. On the other hand, in the bottom up approach, given the explicit content of $6 \mathrm{~d}$ multiplets which satisfy the anomaly cancellations, one should also be able to directly write down the elliptic blowup equations for the elliptic genera of such $6 \mathrm{~d}$ SCFT, as a generalization of the current paper. The two pictures are related by geometric engineering, as the two formalism of blowup equations are related by de-affinisation.

The K-theoretic Nekrasov partition function inspired the study on K-theoretic invariants for general 4-manifolds, specially complex surfaces [17]. Since the elliptic genus of $6 \mathrm{~d}$ $(1,0)$ SCFTs in 5d limit gives K-theoretic Nekrasov partition function, naturally one wonders if elliptic genus can be used to construct some elliptic version of 4-manifold invariants, such as Donaldson invariants. Besides, the Nekrasov partition function is known to relate to $\mathrm{W}$-algebras. In $4 \mathrm{~d}$, the equality between the universal one-instanton Nekrasov partition function and the norm of Gaiotto-Whittaker vectors in W-algebra has been checked in [59]. See proof in [93]. In 5d, the relation between K-theoretic $\mathrm{SU}(N)$ Nekrasov partition function and $q$-deformed $\mathrm{W}_{N}$-algebra was also studied in [94]. It seems natural to extend such relation to $6 \mathrm{~d}$ where the elliptic genus should be related to the elliptic $\mathrm{W}$-algebras. The elliptic W-algebra associated to general Lie algebras is very difficult to study. We hope our exact formula on the elliptic genus could shed some new light. For example, it would be nice to see if one can use elliptic W-algebra to make comparison with our universal one-string elliptic genus formula (3.33) like those comparison done in [59]. One can even ask whether the structure of blowup equations itself can find some origin in pure algebras. See a possible direction [95]. 
One major remaining question is of course how to prove the elliptic blowup equations, or more general, the blowup equations for all local Calabi-Yau in [15, 23, 29]. As the refined BPS invariants for non-compact Calabi-Yau threefolds have been rigorously defined via refined stable pairs in [11], these functional equations for the partition functions are indeed well-formulated mathematical conjectures. See also the definition of refined invariants in [12]. The proof of Göttsche-Nakajima-Yoshioka K-theoretic blowup equations [18] relies deeply on the structure of gauge theories, which may not be exactly suitable for Calabi-Yau setting, as the latter does not necessarily engineer a gauge theory. As emphasized before, the formalism of generalized blowup equations is not sensitive to additional structures of non-compact Calabi-Yau threefolds, be they toric or elliptic. Let us also point out there is even no physical proof for the generalized blowup equations in [15, 23, 29]. In particular, it would be good to see if one can connect the blowup equations with refined holomorphic anomaly equations. Specializing to elliptic blowup equations studied in this paper, we suspect by using the Kac-Weyl character formulas and following the 4d derivation in [59], one may be able to derive the universal $\mathbb{E}_{1}$ formulas (3.33) and the identities from the leading degree of vanishing blowup equations (3.48). We leave these for future studies.

Another major question is how to explain the surprising relation between the elliptic genera of $6 \mathrm{~d}(1,0) \mathrm{SCFT}$ and the Schur indices of $4 \mathrm{~d} \mathcal{N}=2 H_{G}$ theories. Despite the striking relation for rank two cases and even some rank three cases shown in this paper, we do not find the exact formulas connecting the two and three-string elliptic genera and those $L_{G}$ functions like (5.17) in rank one cases found in [37]. To obtain such fascinating formulas for arbitrary rank, it seems one has to answer some questions first. For example, what is the physical meaning for the $L_{G}^{(k)}$ functions? ${ }^{42}$ How to interpret and make use of those nonpertubative symmetries (4.27)? In $4 \mathrm{~d}$ SCFT $H_{G}$, precisely how should the $\beta$-twisted partition function on $T^{2} \times S^{2}$ be related to the superconformal indices on $S^{3} \times S^{1}$ ? And how should the SCFT/VOA correspondence be put in this picture? One possible direction is to look into the localization on the $4 \mathrm{~d}$ backgrounds following the recent works [75-79].

\footnotetext{
${ }^{42}$ Naively one may tempt to identify $L_{G}$ functions as Macdonald indices, since they both have two parameters, and both serve as an unification of Hall-Littlewood indices and Schur indices. However, this seems not ture. For example, the Macdonald index of rank one $H_{E_{7}}$ can be easily obtained by taking limit in the full superconformal index in [48] as

$$
\begin{aligned}
\mathcal{I}_{H_{E_{7}}^{(1)}}^{M}(q, t)= & 1+133 t+\left(134 t q+7371 t^{2}\right)+\left(134 t q^{2}+16149 t^{2} q+238602 t^{3}\right) \\
& +\left(134 t q^{3}+25193 t^{2} q^{2}+819413 t^{3} q+5248750 t^{4}\right)+\ldots
\end{aligned}
$$
}

While the $L_{E_{7}}^{(1)}$ function is determined in [37] as

$$
\begin{aligned}
L_{E_{7}}^{(1)}\left(Q_{\tau}, v\right)= & 1+133 v^{2}+7371 v^{4}+238602 v^{6}+5248750 v^{8}+\ldots \\
& +Q_{\tau}\left(134+16283 v^{2}+835562 v^{4}+\ldots\right)+Q_{\tau}^{2}(31373+\ldots)+\ldots
\end{aligned}
$$

One can see they are indeed not the same, albeit $\mathcal{I}_{H_{E_{7}}^{(1)}}^{M}\left(0, v^{2}\right)=L_{E_{7}}^{(1)}(0, v)$ and $\mathcal{I}_{H_{E_{7}}^{(1)}}^{M}(q, q)=L_{E_{7}}^{(1)}\left(q^{2}, q^{1 / 2}\right)$. 


\section{Acknowledgments}

We thank Babak Haghighat for the early participation of this project. We also thank Christopher Beem, Giulio Bonelli, Michele Del Zotto, Lothar Göttsche, Min-xin Huang, Guglielmo Lockhart, Joonho Kim, Seok Kim, Hiraku Nakajima, Leonardo Rastelli, Alessandro Tanzini, Wenbin Yan, Don Zagier and Rui-Dong Zhu for useful discussion. JG is supported by the Fonds National Suisse, subsidiary 200020-175539 (project "Quantum mechanics, geometry and strings").

\section{A Lie algebraic convention}

We collect some definitions in (affine) Lie algebras and fix our convention used throughout the paper. Given a simple Lie algebar $\mathfrak{g}$ of $\operatorname{rank} \operatorname{rk}(G)$, there are four $\operatorname{rk}(G)$-dimensional lattices of importance, the root and coroot lattices $Q, Q^{\vee}$, the weight and coweight lattices $P, P^{\vee}$. They satisfy

$$
\begin{aligned}
& Q^{\vee} \subset P \subset \mathfrak{h}_{\mathbb{C}}, \\
& Q \subset P^{\vee} \subset \mathfrak{h}_{\mathbb{C}}^{*} .
\end{aligned}
$$

Here $\mathfrak{h}_{\mathbb{C}}, \mathfrak{h}_{\mathbb{C}}^{*} \cong \mathbb{C}^{\text {rk }}$ are the complexified Cartan subalgebra and its dual. They are isomorphic to each other via the natural inner product

$$
\langle\bullet, \bullet\rangle: \mathfrak{h}_{\mathbb{C}}^{*} \times \mathfrak{h}_{\mathbb{C}} \rightarrow \mathbb{C}
$$

The Cartan matrix is then defined by

$$
A_{i j}=\left\langle\alpha_{i}, \alpha_{j}^{\vee}\right\rangle
$$

where $\alpha_{i}$ are simple roots. Consider the invariant bilinear form $(\bullet, \bullet)$ on the coroot lattice $Q^{\vee}$ normalized so that the norm square of the shortest coroot $\theta^{\vee}$ is two. It can be generalized to a bilinear form on $\mathfrak{h}_{\mathbb{C}}$ in which $Q^{\vee}$ is embedded. By the isomorphism between $\mathfrak{h}_{\mathbb{C}}$ and $\mathfrak{h}_{\mathbb{C}}^{*}$, it induces also an invariant bilinear form with the same notation on the latter vector space. With our normalization, the bilinear form satisfies

$$
(k, k)=\frac{1}{2 h_{\mathfrak{g}}^{\vee}} \sum_{\alpha \in \Delta}\langle\alpha, k\rangle^{2}, \quad k \in \mathfrak{h},
$$

where $h_{\mathfrak{g}}^{\vee}$ is the dual Coxeter number, and $\Delta$ the set of all roots.

We also define the fundamental weights $\omega_{i} \in P$ and fundamental coweights $\omega_{i}^{\vee} \in P^{\vee}$ $(i=1, \ldots$, rk $)$ through

$$
\left\langle\alpha_{i}, \omega_{j}\right\rangle=\left\langle\omega_{i}^{\vee}, \alpha_{j}^{\vee}\right\rangle=\delta_{i j} .
$$

They are related to roots and coroots by

$$
\alpha_{i}=\sum_{j=1}^{\mathrm{rk}} A_{i j} \omega_{j}^{\vee}, \quad \alpha_{i}^{\vee}=\sum_{j=1}^{\mathrm{rk}} \omega_{j} A_{j i} .
$$


Most of these definitions can be generalized to the affine Lie algebra $\hat{\mathfrak{g}}$. We add an additional simple root $\alpha_{0}$ satisfying

$$
\alpha_{0}=\alpha_{0}^{\vee}, \quad\left(\alpha_{0}, \alpha_{0}\right)=\left(\alpha_{0}^{\vee}, \alpha_{0}^{\vee}\right)=2 .
$$

The affine Cartan matrix is defined to be

$$
\hat{A}_{I J}=\left\langle\alpha_{I}, \alpha_{J}^{\vee}\right\rangle, \quad I, J=0,1, \ldots, \mathrm{rk},
$$

where $a_{I}, a_{J}^{\vee}$ are the marks and the comarks of the affine Lie algebra $\hat{\mathfrak{g}}$ respectively. The affine Cartan matrix satisfies

$$
\sum_{I=0}^{\mathrm{rk}} a_{I} \hat{A}_{I J}=\sum_{J=0}^{\mathrm{rk}} \hat{A}_{I J} a_{J}^{\vee}=0 .
$$

Note that $\alpha_{0}$ can be written in terms of the longest root $\theta$ and the imaginary root $\delta$, which annihilates anything in $\mathfrak{h}$ or $\mathfrak{h}^{*}$ and has a vanishing norm square, by

$$
\alpha_{0}=\delta-\theta
$$

Similarly we can also define the fundamental weights $\widehat{\omega}_{I}$ and coweights $\widehat{\omega}_{I}^{\vee}$ in the affine Lie algebra $\hat{\mathfrak{g}}$ by

$$
\left\langle\alpha_{I}, \widehat{\omega}_{J}\right\rangle=\left\langle\widehat{\omega}_{I}^{\vee}, \alpha_{J}^{\vee}\right\rangle=\delta_{I J}, \quad I, J=0, \ldots, \mathrm{rk} .
$$

For $i=1, \ldots$, rk the fundamental (co-)weights in $\hat{\mathfrak{g}}$ are related those in $\mathfrak{g}$ by

$$
\begin{aligned}
\widehat{\omega}_{i} & =\omega_{i}+a_{i} \widehat{\omega}_{0}, \\
\widehat{\omega}_{i}^{\vee} & =\omega_{i}^{\vee}+a_{i}^{\vee} \widehat{\omega}_{0}^{\vee},
\end{aligned}
$$

while $\widehat{\omega}_{0}=\widehat{\omega}_{0}^{\vee}$ is imaginary, and it satisfies

$$
\left\langle\alpha_{i}, \widehat{\omega}_{0}\right\rangle=\left\langle\widehat{\omega}_{0}, \alpha_{i}^{\vee}\right\rangle=0, \quad\left(\delta, \widehat{\omega}_{0}\right)=1 .
$$

Using these relations together with (A.7), we find the affine version of (A.7)

$$
\alpha_{I}=\sum_{J=0}^{\mathrm{rk}} \hat{A}_{I J} \widehat{\omega}_{J}^{\vee}+\delta \cdot \delta_{I, 0}, \quad \alpha_{I}^{\vee}=\sum_{J=0}^{\mathrm{rk}} \widehat{\omega}_{J} \hat{A}_{J I}+\delta \cdot \delta_{I, 0} .
$$

\section{B Mirror symmetry for elliptic non-compact Calabi-Yau three-folds}

The prescription in section 2.2 can determine all the triple intersection numbers in the non-compact Calabi-Yau $X$ associated to a minimal 6d SCFT except for the number $\kappa_{\tau \tau \tau}$. Given the non-compactness of $X$ we do not expect all the triple intersection numbers to be computable, and the number $\kappa_{\tau \tau \tau}$ is irrelevant for the blowup equations in any case. Nevertheless we propose here a reasonable normalisation scheme for $\kappa_{\tau \tau \tau}$, which involves a local version of mirror symmetry. We use this normalisation scheme in the example section 4 . 
For the compact Calabi-Yau $\hat{X}$ where $X$ is embedded, one can define for every toric charge $l^{(i)}$ a Picard-Fuchs operator $\hat{\mathcal{L}}_{i}$ which annihilates the homogeneous periods $\hat{\omega}_{0}, \hat{\Pi}_{i}^{(1)}$, $\hat{\Pi}_{i}^{(2)}$. In the decompactification limit $z_{\mathrm{de}} \rightarrow 0$, the Picard-Fuchs $(\mathrm{PF})$ operator associated to the (0)-curve in the base vanishes, while the other operators $\mathcal{L}_{I}$ remain well-defined and non-trivial, and they annihilate all the finite homogeneous periods ${ }^{43} \omega_{0}, \omega_{0} t_{I}, \omega_{0} F_{J}$ of the resulting local Calabi-Yau. These operators, however, do not form a PF complete system, in the sense that they have extra independent solutions. To cure this problem, we define in addition the PF operator $\mathcal{L}_{\tau}$ from the toric charge of the elliptic fiber $\tau$ : $(-6,2,3,0, \ldots, 1, \ldots) .{ }^{44}$ It annihilates all the finite homogeneous periods, but not the other superfluous solutions, thus making the PF system complete.

The number $\kappa_{\tau \tau \tau}$ is contained in the homogeneous B-period $\omega_{0} F_{\tau}=\omega_{0} \partial_{\tau} F_{(0,0)}$ which corresponds to the zero section. We find that it is completely fixed by the normalisation condition

$$
\mathcal{L}_{\tau}\left(\omega_{0} \partial_{\tau} F_{(0,0)}\right)=0
$$

Note that the resulting homogeneous B-period is not a solution to the complete PF system. When it is acted upon by the other PF operators it does not vanish but produces $\omega_{0}$ up to a scaling factor, which may have some open string interpretation.

There are several ways to understand this normalisation scheme. Once all the triple intersection numbers are known, the normalised Euler characteristic can be computed by ${ }^{45}$

$$
\chi=\int_{X} c_{3}(X)=\frac{1}{3} \sum_{i j k} \kappa_{i j k} l_{n}^{(i)} l_{n}^{(j)} l_{n}^{(k)},
$$

we list the results of all the minimal 6 CFTs except for the cases of $\mathfrak{n}=3,7$ in table 23 . Note that the calculation of the normalised Euler characteristic for the case $\mathfrak{n}>3$ is different from that for the first three cases. For $\mathfrak{n}>2$ the elliptic singularity is constant over the $-\mathfrak{n}$ curve in the base. Except for the $\mathfrak{n}=3$ case, the resolved geometry can be described as configuration of Hirzebruch surfaces inside the compact CY-3-fold with only even Betti numbers and $\chi(X)=1+b_{2}+b_{4}$. The case of $\mathfrak{n}=3, G=\mathrm{SU}(3)$ is special as the reduction from the compact geometry to the non-compact geometry also involves a flop operation. The geometry of $\mathfrak{n}=2$ has more supersymmetry and odd Betti numbers $b_{0}=b_{4}=1$, $b_{1}=b_{2}=b_{3}=2$. In the case $\mathfrak{n}=1$ we normalise the Euler characteristic using the formula for the compact threefold [96] with $E_{8}$ elliptic fibre type $\chi\left(X_{\text {comp }}\right)=-2 \cdot 30 \times \int_{B} c_{1}^{2}(M)$. The effect of blowing up a $C^{2}=-\mathfrak{n}=1$ curve decreases $\int_{B} c_{1}^{2}(M)$ by one. Hence the contribution of the non-compact geometry should be $\chi(M)=60$.

The Euler characteristics thus computed for the theories with a pure gauge bulk agree with the naive definition in terms of the numbers $b_{n}$ of compact $n$-cycles $\chi=\sum_{n}(-1)^{n} b_{n}$.

\footnotetext{
${ }^{43}$ For these elliptic Calabi-Yau threefolds decompactified in the horizontal direction, the fundamental period $\hat{\omega}_{0}$ does not become a constant but remains a non-trivial holomorphic function $\omega_{0}$.

${ }^{44}$ The charge 1 corresponds to the zero section of the elliptic fibration.

${ }^{45}$ Here it is understood that we omit the toric charge of the (0)-curve in the summation. If one wishes the charge entry associated to the pullback of the base curve (denoted by $S$ in table 2) can also be ignored as it is only nonzero for the (0)-curve. See for instance table 2 for the model with $G=F_{4}$.
} 


\begin{tabular}{|cccccccc|}
\hline $\mathfrak{n}$ & 1 & 2 & 4 & 5 & 6 & 8 & 12 \\
\hline$G$ & - & - & $S O(8)$ & $F_{4}$ & $E_{6}$ & $E_{7}$ & $E_{8}$ \\
$b_{2}$ & 1 & & 6 & 6 & 8 & 9 & 10 \\
$b_{4}$ & 10 & & 5 & 5 & 7 & 8 & 9 \\
$\chi(X)$ & 60 & 0 & 12 & 12 & 16 & 18 & 20 \\
\hline
\end{tabular}

Table 23. The normalised Euler characteristics of the non-compact elliptic Calabi-Yau threefolds associated to minimal 6d SCFTs.

Furthermore, by integrating the B-period $\partial_{\tau} F_{(0,0)}$ we can compute the genus 0 GW invariants in the $\tau$ direction, which should be the same as the Euler characteristics. We checked this for the $\mathfrak{n}=1,2,5,6$ models. ${ }^{46}$

In the following, we illustrate this idea with two examples.

$\mathfrak{n}=1$.

\begin{tabular}{|c|c|c|c|c|c|c|c|}
\hline$D$ & \multicolumn{4}{|c|}{$\nu_{i}^{*}$} & $l^{(1)}$ & $l^{(2)}$ & $l^{(3)}$ \\
\hline$D_{0}$ & 0 & 0 & 0 & 0 & -6 & 0 & 0 \\
\hline$D_{1}$ & -1 & 0 & 0 & 0 & 2 & 0 & 0 \\
\hline$D_{2}$ & 0 & -1 & 0 & 0 & 3 & 0 & 0 \\
\hline$S^{\prime}$ & 2 & 3 & 0 & -1 & 0 & -1 & 1 \\
\hline$K$ & 2 & 3 & 0 & 0 & 1 & -1 & -2 \\
\hline$F$ & 2 & 3 & -1 & -1 & 0 & 1 & 0 \\
\hline$S$ & 2 & 3 & 0 & 1 & 0 & 0 & 1 \\
\hline$F$ & 2 & 3 & 1 & 0 & 0 & 1 & 0 \\
\hline
\end{tabular}

The Picard-Fuchs operators of the compact geometry are

$$
\begin{aligned}
& \hat{\mathcal{L}}_{1}=\theta_{1}\left(\theta_{1}-\theta_{2}-2 \theta_{3}\right)-12 z_{1}\left(6 \theta_{1}+1\right)\left(6 \theta_{1}+5\right), \\
& \hat{\mathcal{L}}_{2}=\theta_{2}^{2}-z_{2}\left(\theta_{1}-\theta_{2}-2 \theta_{3}\right)\left(\theta_{3}-\theta_{2}\right) \\
& \hat{\mathcal{L}}_{3}=\theta_{3}\left(\theta_{3}-\theta_{2}\right)-z_{3}\left(\theta_{1}-\theta_{2}-2 \theta_{3}-1\right)\left(\theta_{1}-\theta_{2}-2 \theta_{3}\right),
\end{aligned}
$$

where $\theta_{i}:=z_{i} \frac{\partial}{\partial z_{i}}$. Denote $\hat{F}_{0}$ the compact genus zero free energy, we have the periods:

$$
\begin{aligned}
& \hat{X}_{0}=\hat{\omega}_{0}, \quad \hat{X}_{1}=\hat{\omega}_{0} t_{1}, \quad \hat{X}_{2}=\hat{\omega}_{0} t_{2}, \\
& \hat{X}_{3}=\left.\left(\frac{\partial}{\partial \rho_{2} \partial \rho_{3}}+\frac{1}{2} \frac{\partial}{\partial \rho_{3}^{2}}\right) \hat{\omega}_{0}(\rho)\right|_{\rho=0}, \\
& \hat{X}_{4}=\left.\left(-\frac{1}{2} \frac{\partial}{\partial \rho_{1}^{2}}-\frac{\partial}{\partial \rho_{1} \partial \rho_{2}}\right) \hat{\omega}_{0}(\rho)\right|_{\rho=0}, \\
& \hat{X}_{5}=\left.\left(-\frac{\partial}{\partial \rho_{1}^{2}}-\frac{\partial}{\partial \rho_{1} \partial \rho_{3}}\right) \hat{\omega}_{0}(\rho)\right|_{\rho=0},
\end{aligned}
$$

\footnotetext{
${ }^{46}$ For the remaining models the first non-vanishing invariants appear at very high degree and we fail to obtain them within a reasonable period of time.
} 
and another period with triple logarithmic singularity. Here $\hat{\omega}_{0}(\rho)$ is the deformed fundamental period

$$
\hat{\omega}_{0}(\rho)=\sum_{\substack{n_{1}, n_{2}, n_{3} \in \mathbb{Z}_{\geq 0} \\ x_{i}=n_{i}+\rho_{i}}} \frac{\Gamma\left(x_{1}+1\right)}{\Gamma\left(x_{1}+1\right) \Gamma\left(x_{1}+1\right) \Gamma\left(x_{1}-x_{2}-2 x_{3}+1\right) \Gamma\left(-x_{2}+x_{3}+1\right) \Gamma\left(x_{3}+1\right) \Gamma\left(x_{2}+1\right)^{2}} z_{1}^{x_{1}} z_{2}^{x_{2}} z_{3}^{x_{3}},
$$

and $\hat{\omega}_{0}=\hat{\omega}_{0}(0)$. The non-compact geometry is related to the compact geometry by setting $z_{\mathrm{dc}}:=z_{3} \rightarrow 0$. Then the Picard-Fuchs operator $\hat{\mathcal{L}}_{3}$ vanishes while the other two become:

$$
\begin{aligned}
& \mathcal{L}_{1}=\theta_{1}\left(\theta_{1}-\theta_{2}\right)-12 z_{1}\left(6 \theta_{1}+1\right)\left(6 \theta_{1}+5\right), \\
& \mathcal{L}_{2}=\theta_{2}^{2}+z_{2}\left(\theta_{1}-\theta_{2}\right) \theta_{2} .
\end{aligned}
$$

The deformed fundamental period $\hat{\omega}_{0}(\rho)$ becomes $\omega_{0}(\rho)=\left.\hat{\omega}_{0}(\rho)\right|_{z_{3} \rightarrow 0}$. There are one period $X_{0}=\omega_{0}=\left.\hat{\omega}_{0}\right|_{z_{3} \rightarrow 0}$ without singularity, two periods with logarithmic singularities

$$
X_{1}=\left.\hat{X}_{1}\right|_{z_{3} \rightarrow 0}, \quad X_{2}=\left.\hat{X}_{2}\right|_{z_{3} \rightarrow 0},
$$

one with double logarithmic singularities

$$
X_{3}=\left.\left(-\frac{1}{2} \frac{\partial}{\partial \rho_{1}^{2}}-\frac{\partial}{\partial \rho_{1} \partial \rho_{2}}\right) \omega_{0}(\rho)\right|_{\rho=0}=\omega_{0} \frac{\partial}{\partial t_{2}} F_{0}=\left.\hat{X}_{3}\right|_{z_{3} \rightarrow 0},
$$

and no solution with triple logarithmic singularities.

The reason we cannot fix the $\tau$ terms is because we do not know the $\tau=t_{1}$ derivative of the free energy, however, there is an interesting "period" $X_{4}=\omega_{0} \frac{\partial}{\partial \tau} F_{(0,0)}$ which satisfies (up to all the orders we have checked)

$$
\mathcal{L}_{1} X_{4}=0, \quad \mathcal{L}_{2} X_{4}=2 \omega_{0}
$$

Note that $\mathcal{L}_{1}$ is precisely the PF operator in the $\tau$-direction. If $X_{4}$ is a special period, we can integrate both $X_{3}, X_{4}$ and fix the full triple intersection ring

$$
\mathcal{R}=-J_{1}^{3}-J_{2} J_{1}^{2}-J_{2}^{2} J_{1}
$$

and then proceed to compute the Euler number $\chi=60$ as well as $\int c_{2} J_{1}=-10$, $\int c_{2} J_{1}=-12$ using (B.2) and (2.11).

Note that $X_{4}$ indeed descends from a period of the compact geometry. In the noncompact geometry it is the properly normalised integral over a non-compact cycle in the mirror Calabi-Yau.

$\mathfrak{n}=\mathbf{5}$. The computation for the geometry with $\mathfrak{n}=1$ is kind of trivial. Let us now consider a more complicated model with $\mathfrak{n}=5$. Notice in the compact cases, we embed our elliptic Calabi-Yau 3-fold into a toric variety described by a reflexive polytope and its star triangulation. Then the Mori cone generators $l^{(i)}$, which are also known as toric charges, are related to the star triangulation directly. In the de-compactification limit, a point of the polytope is missing, leaving a non-reflexive polytope. The dual polytope now in principle have infinite size. As depicted in section 2.2, the limit happens to take the 
variable $z_{\mathrm{dc}}$ to 0 . This is equivalent to deleting one Mori generator, and keeping the others. From the polytope point of view, we delete a sub-polytope from it, and keep the same triangulation on the remaining part. We may assume that the standard method of mirror symmetry for a compact hyper-surface embedded in a compact toric variety still holds.

For now, we try to triangulate the non-reflexive polytope, it has 16 star triangulations. For one of them, the toric charges are $l^{(i)}, i=1,2,3,4,6,7$ in table 2 , we say the associated curves form the toric basis of compact curves. One can in principle write down the PicardFuchs equations, and then try to find solutions. For this model, it is possible to change the variables of complex structure parameters $z_{i}$ so that the solutions do not change, and the mirror maps have expansions with positive powers of $z_{i}$. The charges of the new basis $l_{F_{4}}^{(i)}$ can be found in table 2, which correspond exactly to nodes in the Dynkin diagram. Then the complete Picard-Fuchs operators are given in (B.12). There are five B-periods solved from these operators, and an extra one $X_{0} \frac{\partial}{\partial \tau} F_{(0,0)}$ annihilated by all the operators except for $\mathcal{L}_{b}$, with $\mathcal{L}_{b}\left(\omega_{0} \frac{\partial}{\partial \tau} F_{(0,0)}\right) \sim \omega_{0}$. The Euler number can be predicted from the $\tau$ direction genus zero invariant as 12 .

$$
\begin{aligned}
\mathcal{L}_{0}= & \theta_{1}\left(\theta_{1}-2 \theta_{2}+\theta_{3}-\theta_{6}\right)-z_{1}\left(2 \theta_{1}-\theta_{2}\right)\left(2 \theta_{1}-\theta_{2}+1\right), \\
\mathcal{L}_{1}= & -\left(2 \theta_{1}-\theta_{2}\right)\left(\theta_{2}-2 \theta_{3}+\theta_{4}-\theta_{6}\right)-z_{2}\left(\theta_{1}-2 \theta_{2}+\theta_{3}-\theta_{6}-1\right)\left(\theta_{1}-2 \theta_{2}+\theta_{3}-\theta_{6}\right), \\
\mathcal{L}_{2}= & \left(2 \theta_{3}-2 \theta_{4}+\theta_{5}-1\right)\left(2 \theta_{3}-2 \theta_{4}+\theta_{5}\right)\left(\theta_{1}-2 \theta_{2}+\theta_{3}-\theta_{6}\right), \\
& -2 z_{3}\left(2 \theta_{3}+1\right)\left(\theta_{2}-2 \theta_{3}+\theta_{4}-\theta_{6}-1\right)\left(\theta_{2}-2 \theta_{3}+\theta_{4}-\theta_{6}\right), \\
\mathcal{L}_{3}= & \left(\theta_{4}-2 \theta_{5}\right)\left(\theta_{2}-2 \theta_{3}+\theta_{4}-\theta_{6}\right)-z_{4}\left(2 \theta_{3}-2 \theta_{4}+\theta_{5}-1\right)\left(2 \theta_{3}-2 \theta_{4}+\theta_{5}\right), \\
\mathcal{L}_{4}= & \theta_{5}\left(2 \theta_{3}-2 \theta_{4}+\theta_{5}\right)-z_{5}\left(\theta_{4}-2 \theta_{5}-1\right)\left(\theta_{4}-2 \theta_{5}\right), \\
\mathcal{L}_{b}= & \theta_{6}^{2}-z_{6}\left(\theta_{1}-2 \theta_{2}+\theta_{3}-\theta_{6}\right)\left(\theta_{2}-2 \theta_{3}+\theta_{4}-\theta_{6}\right), \\
\mathcal{L}_{\tau}= & \theta_{1}\left(\theta_{5}-1\right) \theta_{5}-8 z_{1} z_{2}^{2} z_{3}^{3} z_{4}^{4} z_{5}^{2}\left(2 \theta_{3}+1\right)\left(2 \theta_{3}+3\right)\left(2 \theta_{3}+5\right) .
\end{aligned}
$$

\section{Geometric data}

We express here the Mori cone generators of the elliptic non-compact Calabi-Yau threefolds $X$ associated to the minimal 6d SCFTs in terms of the Mori cone generators $l^{(i)}$ of the compact Calabi-Yau $\hat{X}$ given in [8].

$\boldsymbol{G}=\mathbf{S O}(\mathbf{8}) . \quad l^{(5)}$ is the direction of decompactification.

$$
\begin{aligned}
& \Sigma_{b}=l^{(4)}, \quad \Sigma_{0}=l^{(6)}, \quad \Sigma_{1}=2 l^{(1)}+l^{(6)}+2 l^{(7)}, \\
& \Sigma_{2}=2 l^{(1)}+l^{(6)}, \Sigma_{3}=l^{(3)}+l^{(6)}, \Sigma_{4}=l^{(1)}+l^{(2)}+2 l^{(7)} .
\end{aligned}
$$

$\boldsymbol{G}=\boldsymbol{F}_{\mathbf{4}} . \quad l^{(5)}$ is the direction of decompactification.

$$
\Sigma_{b}=l^{(4)}, \quad \Sigma_{0}=l^{(3)}, \quad \Sigma_{1}=l^{(2)}+l^{(6)}+2 l^{(7)}, \quad \Sigma_{2}=l^{(1)}, \quad \Sigma_{3}=l^{(6)}, \quad \Sigma_{4}=l^{(7)} .
$$

$\boldsymbol{G}=\boldsymbol{E}_{\mathbf{6}} \cdot l^{(3)}$ is the direction of decompactifiction.

$$
\begin{array}{llll}
\Sigma_{b}=l^{(5)}, \quad \Sigma_{0}=l^{(4)}, & \Sigma_{1}=l^{(1)}+l^{(6)}+2 l^{(7)}+2 l^{(9)}, & \Sigma_{2}=l^{(8)}+l^{(9)}, \\
\Sigma_{3}=l^{(6)}+2 l^{(7)}+l^{(8)}, & \Sigma_{4}=l^{(2)}+l^{(7)}+l^{(8)}, \quad \Sigma_{5}=l^{(6)}, & \Sigma_{6}=l^{(7)}+l^{(9)} .
\end{array}
$$


$\boldsymbol{G}=\boldsymbol{E}_{\mathbf{7}} . \quad l^{(4)}$ is the direction of decompactification.

$$
\begin{aligned}
& \Sigma_{b}=l^{(3)}, \quad \Sigma_{0}=l^{(5)}, \quad \Sigma_{1}=l^{(6)}, \quad \Sigma_{2}=l^{(2)}+l^{(7)}+2 l^{(8)}+l^{(9)}, \quad \Sigma_{3}=l^{(1)}+l^{(7)}+l^{(10)}, \\
& \Sigma_{4}=l^{(7)}, \quad \Sigma_{5}=l^{(8)}+l^{(10)}, \quad \Sigma_{6}=l^{(9)}, \quad \Sigma_{7}=l^{(7)}+2 l^{(8)}+l^{(9)} .
\end{aligned}
$$

$\boldsymbol{G}=\boldsymbol{E}_{\mathbf{8}} . \quad l^{(3)}$ is the direction fo decompactification.

$$
\begin{array}{llll}
\Sigma_{b}=l^{(8)}, & \Sigma_{0}=l^{(4)}, & \Sigma_{1}=l^{(5)}, & \Sigma_{2}=l^{(6)}, \quad \Sigma_{3}=l^{(7)}, \quad \Sigma_{4}=l^{(1)}+l^{(2)}+l^{(10)}+2 l^{(11)}, \\
\Sigma_{5}=l^{(9)}, & \Sigma_{6}=l^{(10)}, & \Sigma_{7}=l^{(11)}, & \Sigma_{8}=l^{(1)}+l^{(10)}+2 l^{(11)} .
\end{array}
$$

\section{Useful identities}

Jacobi theta functions with characteristics are defined as

$$
\begin{aligned}
& \theta_{1}^{[a]}(\tau, z)=-\mathrm{i} \sum_{k \in \mathbb{Z}}(-1)^{k+a} Q_{\tau}^{(k+1 / 2+a)^{2} / 2} Q_{z}^{k+1 / 2+a}, \\
& \theta_{2}^{[a]}(\tau, z)=\sum_{k \in \mathbb{Z}} Q_{\tau}^{(k+1 / 2+a)^{2} / 2} Q_{z}^{k+1 / 2+a} \\
& \theta_{3}^{[a]}(\tau, z)=\sum_{k \in \mathbb{Z}} Q_{\tau}^{(k+a)^{2} / 2} Q_{z}^{k+a} \\
& \theta_{4}^{[a]}(\tau, z)=\sum_{k \in \mathbb{Z}}(-1)^{k+a} Q_{\tau}^{(k+a)^{2} / 2} Q_{z}^{k+a}
\end{aligned}
$$

which satisfy the well-known addition formulas

$$
\begin{aligned}
& \theta_{3}^{\left[a_{1}\right]}\left(\tau, z_{1}\right) \theta_{3}^{\left[a_{2}\right]}\left(\tau, z_{2}\right)=\sum_{i=2,3} \theta_{i}^{\left[\frac{a_{1}+a_{2}}{2}\right]}\left(2 \tau, z_{1}+z_{2}\right) \theta_{i}^{\left[\frac{a_{1}-a_{2}}{2}\right]}\left(2 \tau, z_{1}-z_{2}\right), \\
& \theta_{4}^{\left[a_{1}\right]}\left(\tau, z_{1}\right) \theta_{4}^{\left[a_{2}\right]}\left(\tau, z_{2}\right)=\sum_{i=1,4} \theta_{i}^{\left[\frac{a_{1}+a_{2}}{2}\right]}\left(2 \tau, z_{1}+z_{2}\right) \theta_{i}^{\left[\frac{a_{1}-a_{2}}{2}\right]}\left(2 \tau, z_{1}-z_{2}\right) .
\end{aligned}
$$

Jacobi theta function $\theta_{1}$ can be defined as triple products

$$
\theta_{1}(\tau, z)=\mathrm{i} Q_{\tau}^{\frac{1}{12}} Q_{z}^{-\frac{1}{2}} \eta(\tau) \prod_{n=1}^{\infty}\left(1-Q_{z} Q_{\tau}^{n-1}\right)\left(1-\frac{Q_{\tau}^{n}}{Q_{z}}\right)
$$

which satisfies the quasi-periodicity

$$
\begin{aligned}
& \theta_{1}(\tau, z+1)=-\theta_{1}(\tau, z), \\
& \theta_{1}(\tau, z+\tau)=-Q_{\tau}^{-1 / 2} Q_{z}^{-1} \theta_{1}(\tau, z), \\
& \theta_{1}(\tau, z-\tau)=-Q_{\tau}^{-1 / 2} Q_{z} \theta_{1}(\tau, z) .
\end{aligned}
$$

For a cluster of refined BPS invariants $N_{(0, k)}^{k}=1$ for all $k \geq 0$, the total contribution to BPS partition function is

$$
\sum_{k=0}^{\infty} \frac{Q^{k} \chi_{2 k+1}\left(\left(q_{1} q_{2}\right)^{1 / 2}\right)}{\left(q_{1}^{1 / 2}-q_{1}^{-1 / 2}\right)\left(q_{2}^{1 / 2}-q_{2}^{-1 / 2}\right)}=\frac{1+Q}{\left(q_{1}^{1 / 2}-q_{1}^{-1 / 2}\right)\left(q_{2}^{1 / 2}-q_{2}^{-1 / 2}\right)\left(1-Q q_{1} q_{2}\right)\left(1-Q q_{1}^{-1} q_{2}^{-1}\right)} .
$$


Similiarly, for a cluster of refined BPS invariants $N_{(0, k+1 / 2)}^{k}=-1$ for all $k \geq 0$, the total contribution to BPS partition function is

$$
\sum_{k=0}^{\infty} \frac{Q^{k} \chi_{2 k+2}\left(\left(q_{1} q_{2}\right)^{1 / 2}\right)}{\left(q_{1}^{1 / 2}-q_{1}^{-1 / 2}\right)\left(q_{2}^{1 / 2}-q_{2}^{-1 / 2}\right)}=\frac{\left(q_{1} q_{2}\right)^{1 / 2}+\left(q_{1} q_{2}\right)^{-1 / 2}}{\left(q_{1}^{1 / 2}-q_{1}^{-1 / 2}\right)\left(q_{2}^{1 / 2}-q_{2}^{-1 / 2}\right)\left(1-Q q_{1} q_{2}\right)\left(1-Q q_{1}^{-1} q_{2}^{-1}\right)}
$$

We also often encounter the case where a cluster of refined BPS invariants $N_{(0, k-1 / 2)}^{k}=$ -1 for all $k \geq 0$ are combined with a "zero" degree invariants $N_{(0,1 / 2)}^{0}=-1$. In such case, the total contribution is

$$
\begin{gathered}
\frac{1}{\left(q_{1}^{1 / 2}-q_{1}^{-1 / 2}\right)\left(q_{2}^{1 / 2}-q_{2}^{-1 / 2}\right)}+\sum_{k=1}^{\infty} \frac{Q^{k} \chi_{2 k}\left(\left(q_{1} q_{2}\right)^{1 / 2}\right)}{\left(q_{1}^{1 / 2}-q_{1}^{-1 / 2}\right)\left(q_{2}^{1 / 2}-q_{2}^{-1 / 2}\right)} \\
=\frac{\left(\left(q_{1} q_{2}\right)^{1 / 2}+\left(q_{1} q_{2}\right)^{-1 / 2}\right)\left(1+Q+Q^{2}-\left(q_{1} q_{2}+q_{1}^{-1} q_{2}^{-1}\right) Q\right)}{\left(q_{1}^{1 / 2}-q_{1}^{-1 / 2}\right)\left(q_{2}^{1 / 2}-q_{2}^{-1 / 2}\right)\left(1-Q q_{1} q_{2}\right)\left(1-Q q_{1}^{-1} q_{2}^{-1}\right)} .
\end{gathered}
$$

In the computation of vector multiplets, we often encounter the following expressions:

$$
\mathrm{PE}\left[\frac{Q}{1-Q_{\tau}}\right]=\prod_{n=0}^{\infty} \frac{1}{1-Q Q_{\tau}^{n}},
$$

and

$$
\mathrm{PE}\left[\left(Q_{z}+\frac{Q_{\tau}}{Q_{z}}\right)\left(\frac{1}{1-Q_{\tau}}\right)\right]=\frac{\mathrm{i} Q_{\tau}^{\frac{1}{12}} Q_{z}^{-\frac{1}{2}} \eta(\tau)}{\theta_{1}(\tau, z)} .
$$

In counting the total index quadratic form of the contribution from vector multiplets, we often encounter the following expression:

$$
\operatorname{PE}\left(-\left(B l_{(0,1 / 2, R)}\left(q_{1}, q_{2}\right) Q_{z}+B l_{(0,1 / 2,-R)}\left(q_{1}, q_{2}\right) \frac{Q_{\tau}}{Q_{z}}\right)\left(\frac{1}{1-Q_{\tau}}\right)\right) .
$$

Here

$$
B l_{\left(j_{l}, j_{r}, R\right)}\left(q_{1}, q_{2}\right)=f_{\left(j_{l}, j_{r}\right)}\left(q_{1}, q_{2} / q_{1}\right) q_{1}^{R}+f_{\left(j_{l}, j_{r}\right)}\left(q_{1} / q_{2}, q_{2}\right) q_{2}^{R}-f_{\left(j_{l}, j_{r}\right)}\left(q_{1}, q_{2}\right),
$$

and

$$
f_{\left(j_{l}, j_{r}\right)}\left(q_{1}, q_{2}\right)=\frac{\chi_{j_{l}}\left(q_{L}\right) \chi_{j_{r}}\left(q_{R}\right)}{\left(q_{1}^{1 / 2}-q_{1}^{-1 / 2}\right)\left(q_{2}^{1 / 2}-q_{2}^{-1 / 2}\right)} .
$$

Supposing $R \geq 2$, the expression (D.12) can be written as

$$
\begin{aligned}
& \prod_{\substack{m, n \geq 0 \\
m+n \leq R-1}} \frac{\mathrm{i} Q_{\tau}^{1 / 12} \eta\left(Q_{z} q_{1}^{m} q_{2}^{n}\right)^{-1 / 2}}{\theta_{1}\left(z+m \epsilon_{1}+n \epsilon_{2}\right)} \prod_{\substack{m, n \geq 0 \\
m+n \leq R-2}} \frac{\mathrm{i} Q_{\tau}^{1 / 12} \eta\left(Q_{z} q_{1}^{m+1} q_{2}^{n+1}\right)^{-1 / 2}}{\theta_{1}\left(z+(m+1) \epsilon_{1}+(n+1) \epsilon_{2}\right)} \\
= & \left(\mathrm{i} Q_{\tau}^{1 / 12} Q_{z}^{-1 / 2}\right)^{R^{2}}\left(q_{1} q_{2}\right)^{-\frac{(R-1) R(R+1)}{6}} \prod_{\substack{m, n \geq 0 \\
m+n \leq R-1}} \frac{\eta}{\theta_{1}\left(z+m \epsilon_{1}+n \epsilon_{2}\right)} \prod_{\substack{m, n \geq 0 \\
m+n \leq R-2}} \frac{\eta}{\theta_{1}\left(z+(m+1) \epsilon_{1}+(n+1) \epsilon_{2}\right)}
\end{aligned}
$$


We normally denote the modular part of (D.12), i.e. those $\theta_{1}$ and $\eta$ functions in the above expression together as $\breve{\theta}$. The index quadratic form of $\breve{\theta}$ can be computed as

$$
\operatorname{Ind}_{\breve{\theta}}(z, R)=-\frac{R^{2} z^{2}}{2}-\frac{(R-1) R(R+1)}{3} z\left(\epsilon_{1}+\epsilon_{2}\right)-\frac{(R-1) R^{2}(R+1)}{12}\left(\epsilon_{1}^{2}+\epsilon_{1} \epsilon_{2}+\epsilon_{2}^{2}\right),
$$

which actually holds for all $R \in \mathbb{Z}$. See more details in the appendix A in [25].

\section{E Relation with modular ansatz}

In this appendix, we show how the modular ansatz, or its denominator to be specific, for the elliptic genus emerges from our exact formulas. Simply speaking, the denominator of the modular ansatz comes from suming over all $\alpha^{\vee}$ with a fixed norm square in the recursion formula.

It was proposed in $[24,37]$ the $k$-string elliptic genus satifies the following ansatz

$$
\mathbb{E}_{\widetilde{h}_{G}^{(k)}}\left(\tau, \epsilon_{1}, \epsilon_{2}, m_{\alpha}\right)=\frac{\mathcal{N}_{G, k}\left(\tau, \epsilon_{1}, \epsilon_{2}, m_{\alpha}\right)}{\mathcal{D}_{G, k}\left(\tau, \epsilon_{1}, \epsilon_{2}, m_{\alpha}\right)},
$$

where both the numerator and the denominator are Weyl invariant Jacobi forms. Furthermore the denominator has the following unique structure as a Weyl invariant Jacobi form which reproduces the poles of the Hilbert series of the moduli space of $k G$-gauge instantons [43] and the correct leading order of $Q_{\tau}$ :

$$
\mathcal{D}_{G, k}=\eta(\tau)^{4 k h_{G}^{\vee}} \prod_{i=1}^{k} \varphi_{-1, \frac{1}{2}}\left(i \epsilon_{1}\right) \varphi_{-1, \frac{1}{2}}\left(i \epsilon_{2}\right) \tilde{D}_{G, k}
$$

with the gauge group related factor

$$
\tilde{\mathcal{D}}_{G, k}=\prod_{\alpha \in \Delta} \prod_{i=1}^{k} \prod_{\ell=0}^{i-1} \varphi_{-1, \frac{1}{2}}\left((i+1) \epsilon_{+}+(i-1-2 \ell) \epsilon_{-}+m_{\alpha}\right),
$$

multiplying over the set of roots. Later [97] claims that $\mathbb{E}_{k}$ has actually fewer poles and as a consequence the denominator is smaller (see also [59]). It can be written as ${ }^{47}$

$$
\mathcal{D}_{G, k}^{\mathrm{red}}=\eta(\tau)^{4 k h_{G}^{\vee}} \prod_{i=1}^{k} \varphi_{-1, \frac{1}{2}}\left(i \epsilon_{1}\right) \varphi_{-1, \frac{1}{2}}\left(i \epsilon_{2}\right) \tilde{\mathcal{D}}_{G, k}^{\mathrm{red}}
$$

with the gauge group related factor

$$
\tilde{\mathcal{D}}_{G, k}^{\mathrm{red}}=\prod_{\alpha \in \Delta^{l}} \mathcal{D}_{k, \alpha}^{\mathrm{SU}(2)}(\tau, m) \prod_{\alpha \in \Delta^{s}} \mathcal{D}_{\left\lfloor k / c_{\alpha}\right\rfloor, \alpha}^{\mathrm{SU}(2)}(\tau, m),
$$

where $\Delta^{l}, \Delta^{s}$ are the set of long roots and short roots respectively, the constants $c_{\alpha}$ are

$$
\begin{array}{ll}
c_{\alpha}=2 & \text { if } G=\operatorname{Sp}(N), \operatorname{SO}(2 N+1), F_{4}, \\
c_{\alpha}=3 & \text { if } G=G_{2},
\end{array}
$$

\footnotetext{
${ }^{47}$ Here "red" means "reduced", i.e. the number of poles reduces. The "tilde" and "reduced" in this section should not be confused with them in the main text where them mean a free hypermultiplet is coupled or decoupled.
} 
and that

$$
\mathcal{D}_{k, \alpha}^{\mathrm{SU}(2)}(\tau, m)=\prod_{\substack{a, b \leq k \\ a, b>0}} \varphi_{-1, \frac{1}{2}}\left(a \epsilon_{1}+b \epsilon_{2}+m_{\alpha}\right) .
$$

To see that $\mathcal{D}_{G, k}^{\text {red }}$ is actually smaller than $\mathcal{D}_{G, k}$, we spell out explicitly the components of $\tilde{\mathcal{D}}_{G, k}, \tilde{\mathcal{D}}_{G, k}^{\text {red }}$ for some small values of $k$. To be concrete, we take the model of $\mathfrak{n}=5$ with $G=F_{4}$. On the one hand,

$$
\begin{aligned}
\tilde{\mathcal{D}}_{1}= & \prod_{\alpha \in \Delta} \varphi_{-1, \frac{1}{2}}\left(\epsilon_{1}+\epsilon_{2}+m_{\alpha}\right), \\
\tilde{\mathcal{D}}_{2}= & \prod_{\alpha \in \Delta} \varphi_{-1, \frac{1}{2}}\left(\epsilon_{1}+\epsilon_{2}+m_{\alpha}\right) \varphi_{-1, \frac{1}{2}}\left(2 \epsilon_{1}+\epsilon_{2}+m_{\alpha}\right) \varphi_{-1, \frac{1}{2}}\left(\epsilon_{1}+2 \epsilon_{2}+m_{\alpha}\right), \\
\tilde{\mathcal{D}}_{3}= & \prod_{\alpha \in \Delta} \varphi_{-1, \frac{1}{2}}\left(\epsilon_{1}+\epsilon_{2}+m_{\alpha}\right) \varphi_{-1, \frac{1}{2}}\left(2 \epsilon_{1}+\epsilon_{2}+m_{\alpha}\right) \varphi_{-1, \frac{1}{2}}\left(\epsilon_{1}+2 \epsilon_{2}+m_{\alpha}\right) \\
& \varphi_{-1, \frac{1}{2}}\left(3 \epsilon_{1}+\epsilon_{2}+m_{\alpha}\right) \varphi_{-1, \frac{1}{2}}\left(2 \epsilon_{1}+2 \epsilon_{2}+m_{\alpha}\right) \varphi_{-1, \frac{1}{2}}\left(\epsilon_{1}+3 \epsilon_{2}+m_{\alpha}\right) .
\end{aligned}
$$

On the other hand,

$$
\begin{aligned}
\tilde{\mathcal{D}}_{1}^{\text {red }}= & \prod_{\alpha \in \Delta^{l}} \varphi_{-1, \frac{1}{2}}\left(\epsilon_{1}+\epsilon_{2}+m_{\alpha}\right) \\
\tilde{\mathcal{D}}_{2}^{\text {red }}= & \prod_{\alpha \in \Delta^{s}} \varphi_{-1, \frac{1}{2}}\left(\epsilon_{1}+\epsilon_{2}+m_{\alpha}\right) \times \\
& \prod_{\alpha \in \Delta^{l}} \varphi_{-1, \frac{1}{2}}\left(\epsilon_{1}+\epsilon_{2}+m_{\alpha}\right) \varphi_{-1, \frac{1}{2}}\left(2 \epsilon_{1}+\epsilon_{2}+m_{\alpha}\right) \varphi_{-1, \frac{1}{2}}\left(\epsilon_{1}+2 \epsilon_{2}+m_{\alpha}\right), \\
\tilde{\mathcal{D}}_{3}^{\text {red }}= & \prod_{\alpha \in \Delta^{s}} \varphi_{-1, \frac{1}{2}}\left(\epsilon_{1}+\epsilon_{2}+m_{\alpha}\right) \times \\
& \prod_{\alpha \in \Delta^{l}} \varphi_{-1, \frac{1}{2}}\left(\epsilon_{1}+\epsilon_{2}+m_{\alpha}\right) \varphi_{-1, \frac{1}{2}}\left(2 \epsilon_{1}+\epsilon_{2}+m_{\alpha}\right) \varphi_{-1, \frac{1}{2}}\left(\epsilon_{1}+2 \epsilon_{2}+m_{\alpha}\right) \\
& \varphi_{-1, \frac{1}{2}}\left(3 \epsilon_{1}+\epsilon_{2}+m_{\alpha}\right) \varphi_{-1, \frac{1}{2}}\left(\epsilon_{1}+3 \epsilon_{2}+m_{\alpha}\right) .
\end{aligned}
$$

We will demonstrate that our recursion formulas (3.32) are consistent with (E.4) and (E.5) rather than (E.2), (E.3).

Let us first take a look at the case of $k=1$ where the recursion formulas (3.32) simply read

$$
\mathbb{E}_{1}=\sum_{\left\|\alpha^{\vee}\right\|^{2}=2}(-1)^{\mid \alpha^{\vee}} \frac{D_{\{1,0,0\}}^{\alpha^{\vee}}}{D_{1}} A_{\alpha^{\vee}}
$$

We consider the poles contributed by each component. Suppose we choose three unity $r$ fields with $a_{1,2,3}$, which differ from each other by $a_{i}-a_{j}=s_{i j} / \mathfrak{n}$. According to the requirement for $a_{1,2,3}$, we know that all $s_{i j}$ are intergers and $0<\left|s_{i j}\right|<n$. Using (D.2), it is not difficult to show that both $D_{1,0,0}^{\alpha \vee}$ and $D_{1}$ contain the zero $\epsilon_{1}-\epsilon_{2}=0$ of order $\min \left(\left|s_{i j}\right|, n-\left|s_{i j}\right|\right)$. For example, the minor

$$
\Delta_{1,2}=\operatorname{det}\left(\begin{array}{cc}
\theta_{3}^{\left[a_{1}\right]}\left(n \tau,-2 \epsilon_{1}+(n-2) \epsilon_{2}\right) & \theta_{3}^{\left[a_{1}\right]}\left(n \tau,(n-2) \epsilon_{1}-2 \epsilon_{2}\right) \\
\theta_{3}^{\left[a_{2}\right]}\left(n \tau,-2 \epsilon_{1}+(n-2) \epsilon_{2}\right) & \theta_{3}^{\left[a_{2}\right]}\left(n \tau,(n-2) \epsilon_{1}-2 \epsilon_{2}\right)
\end{array}\right),
$$


can be rewritten as

$$
\Delta_{1,2}=\sum_{i=2,3} \theta_{i}^{\left[\frac{a_{1}-a_{2}}{2}\right]}\left(2 n \tau, n\left(\epsilon_{2}-\epsilon_{1}\right)\right)-\theta_{i}^{\left[\frac{a_{1}-a_{2}}{2}\right]}\left(2 n \tau, n\left(\epsilon_{1}-\epsilon_{2}\right)\right),
$$

which clearly contain zeros $\epsilon_{1}-\epsilon_{2}=0$ of order $\min \left(\left|s_{i j}\right|, n-\left|s_{i j}\right|\right)$. Now by the universality argument in section 3.2, we can choose arbitrary three $a_{1,2,3}$ in recursion formulas. Let us choose three successive ones with $a_{3}-a_{2}=a_{2}-a_{1}=1 / \mathfrak{n}$. Both $D_{1,0,0}^{\alpha \vee}$ and $D_{1}$ have the simple zero $\epsilon_{1}-\epsilon_{2}=0$. In fact, more is true, the determinant

$$
D\left(z_{1}, z_{2}, z_{3}\right)=\operatorname{det}\left(\begin{array}{ccc}
\theta_{i}^{\left[a_{1}\right]}\left(\mathfrak{n} \tau, \mathfrak{n} z_{1}\right) & \theta_{i}^{\left[a_{1}\right]}\left(\mathfrak{n} \tau, \mathfrak{n} z_{2}\right) & \theta_{i}^{\left[a_{1}\right]}\left(\mathfrak{n} \tau, \mathfrak{n} z_{3}\right) \\
\theta_{i}^{\left[a_{2}\right]}\left(\mathfrak{n} \tau, \mathfrak{n} z_{1}\right) & \theta_{i}^{\left[a_{2}\right]}\left(\mathfrak{n} \tau, \mathfrak{n} z_{2}\right) & \theta_{i}^{\left[a_{2}\right]}\left(\mathfrak{n} \tau, \mathfrak{n} z_{3}\right) \\
\theta_{i}^{\left[a_{3}\right]}\left(\mathfrak{n} \tau, \mathfrak{n} z_{1}\right) & \theta_{i}^{\left[a_{3}\right]}\left(\mathfrak{n} \tau, \mathfrak{n} z_{2}\right) & \theta_{i}^{\left[a_{3}\right]}\left(\mathfrak{n} \tau, \mathfrak{n} z_{3}\right)
\end{array}\right)
$$

with characteristics $a_{1,2,3}$ chosen as above and $i=3,4$ always has simple zeros at $z_{1}-z_{2}=$ $z_{2}-z_{3}=z_{3}-z_{1}=0$. Therefore $D_{1,0,0}^{\alpha \vee} / D_{1}$ has zeros/poles

$$
\frac{\left(\epsilon_{1}-\epsilon_{2}\right)\left(m_{\alpha}-\epsilon_{1}\right)\left(m_{\alpha}-\epsilon_{2}\right)}{\left(\epsilon_{1}-\epsilon_{2}\right) \epsilon_{1} \epsilon_{2}},
$$

which can be boosted to the modular object

$$
\frac{\theta_{1}\left(m_{\alpha}-\epsilon_{1}\right) \theta_{1}\left(m_{\alpha}-\epsilon_{2}\right)}{\theta_{1}\left(\epsilon_{1}\right) \theta_{1}\left(\epsilon_{2}\right)} .
$$

The other component in (E.14) for $\mathbb{E}_{1}$ is

$$
A_{\alpha \vee}(m y)=\frac{\eta^{4}}{\theta_{1}\left(m_{\alpha}\right) \theta_{1}\left(m_{\alpha}-\epsilon_{1}\right) \theta_{1}\left(m_{\alpha}-\epsilon_{2}\right) \theta_{1}\left(m_{\alpha}-\epsilon_{1}-\epsilon_{2}\right)} \prod_{\substack{\beta \in \Delta \\ \beta \cdot \alpha^{\vee}=1}} \frac{\eta}{\theta_{1}\left(m_{\beta}\right)},
$$

where $\alpha=\alpha^{\vee} \cdot 2 /\left\|\alpha^{\vee}\right\|^{2}$ in the root associated to the coroot $\alpha^{\vee}$. The components $\theta_{1}\left(m_{\alpha}-\right.$ $\left.\epsilon_{1}\right) \theta_{1}\left(m_{\alpha}-\epsilon_{2}\right)$ cancel with corresponding components in the numerator of $D_{1,0,0}^{\alpha \vee} / D_{1}$. Thus naively $A_{\alpha} \vee$ should contribute poles ${ }^{48} m_{\beta}=0$ for $\beta \in \Delta$ and $m_{\alpha}-\epsilon_{1}-\epsilon_{2}=0$ for $\alpha \in \Delta^{l}$ to $\mathbb{E}_{1}$. The former poles, however, get canceled with some factors in the numerator after performing the summation over short coroots in (E.14), which can either be seen in explicit calculations, ${ }^{49}$ or be argued formally from the Hilbert series of gauge instanton moduli space [43] as well as from $\mathrm{W}$-algebra via AGT correspondence [59]. The remaining genuine poles are $m_{\alpha}-\epsilon_{1}-\epsilon_{2}=0$ for $\alpha \in \Delta^{l}$, which are consistent with the slimmer expression (E.11) rather than (E.8).

The discussion above indicates that the recursion formulas (3.32) for $\mathbb{E}_{k}$ naively contain both genuine poles and spurious poles, while the cancellation of the latter is not obvious. Nevertheless, all true poles should already be visible in the recursion formulas, which allows us to distinguish (E.4) from (E.2). In the following, we will argue in favor of (E.4) by pointing out that extra poles indicated in (E.2) are not present in the recursion formulas (3.32),

\footnotetext{
${ }^{48}$ There are also poles at positions shifted by 1 or $\tau$ of course.

${ }^{49}$ This cancellation can be checked in all the minimal models, not only in the $\mathfrak{n}=5$ model.
} 
and assume along the way that the spurious poles not consistent with either (E.2) or (E.4) cancel automatically. With this comment in mind, let us now look at the denominators of $\mathbb{E}_{2}, \mathbb{E}_{3}$ from the recursion formulas, and consider only relevant poles, the poles which appear also in either (E.2) or (E.4).

The right hand side of (3.32) for $\mathbb{E}_{2}$ has three sectors with

$$
\left(\left\|\alpha^{\vee}\right\|^{2}, d_{1}, d_{2}\right)=(4,0,0),(2,1,0) /(2,0,1),(0,1,1)
$$

respectively. With an explicit calculation in the $\mathfrak{n}=5$ models, we find the following relevant poles in each of the three sectors ${ }^{50}$

\begin{tabular}{|cl|}
\hline$\left(\left\|\alpha^{\vee}\right\|^{2}, d_{1}, d_{2}\right)$ & relevant poles \\
\hline$(4,0,0)$ & $m_{\alpha}-\epsilon_{1}-\epsilon_{2}, \alpha \in \Delta$ \\
$(2,1,0) /(2,0,1)$ & $m_{\alpha}-\epsilon_{1}-\epsilon_{2}, m_{\alpha}-2 \epsilon_{1}-\epsilon_{2}, m_{\alpha}-\epsilon_{1}-2 \epsilon_{2}, \alpha \in \Delta^{l}$ \\
$(0,1,1)$ & - \\
\hline
\end{tabular}

They are clearly consistent with (E.12). Likewise, for $\mathbb{E}_{3}$ there are five sectors on the right hand sie of (3.32), and we find the relevant poles as follows

\begin{tabular}{|cl|}
\hline$\left(\left\|\alpha^{\vee}\right\|^{2}, d_{1}, d_{2}\right)$ & relevant poles \\
\hline$(6,0,0)$ & $m_{\alpha}-\epsilon_{1}-\epsilon_{2}, \alpha \in \Delta ;$ \\
& $m_{\alpha}-2 \epsilon_{1}-\epsilon_{2}, m_{\alpha}-\epsilon_{1}-2 \epsilon_{2}, \alpha \in \Delta^{l}$ \\
$(4,1,0) /(4,0,1)$ & $m_{\alpha}-\epsilon_{1}-\epsilon_{2}, m_{\alpha}-2 \epsilon_{1}-\epsilon_{2}, m_{\alpha}-\epsilon_{1}-2 \epsilon_{2}, \alpha \in \Delta^{l}$ \\
$(2,2,0) /(2,0,2)$ & $m_{\alpha}-\epsilon_{1}-\epsilon_{2}, \alpha \in \Delta ;$ \\
& $m_{\alpha}-2 \epsilon_{1}-\epsilon_{2}, m_{\alpha}-\epsilon_{1}-2 \epsilon_{2}$, \\
& $m_{\alpha}-3 \epsilon_{1}-\epsilon_{2}, m_{\alpha}-\epsilon_{1}-3 \epsilon_{2}, \alpha \in \Delta^{l}$ \\
$(2,1,1)$ & $m_{\alpha}-\epsilon_{1}-\epsilon_{2}, m_{\alpha}-2 \epsilon_{1}-\epsilon_{2}, m_{\alpha}-\epsilon_{1}-2 \epsilon_{2}, \alpha \in \Delta^{l}$ \\
$(0,2,1)$ & $m_{\alpha}-\epsilon_{1}-\epsilon_{2}, \alpha \in \Delta^{l}$ \\
\hline
\end{tabular}

which are consistent with (E.13).

\section{F Elliptic genera}

We record some high $Q_{\tau}$ order results for the reduced elliptic genus here. Recall the $k$-string elliptic genus when expanded with respect to $Q_{\tau}$ can be written as

$$
\mathbb{E}_{h_{G}^{(k)}}\left(v, x, Q_{\tau}, Q_{m_{i}}\right)=v^{k h_{G}^{\vee}-1} Q_{\tau}^{-\left(k h_{G}^{\vee}-1\right) / 6} \sum_{n=0}^{\infty} Q_{\tau}^{n} g_{k, G}^{(n)}\left(v, x, Q_{m_{i}}\right) .
$$

We are interested in the $v$-expansion of $g_{k, G}^{(n)}\left(v, x, Q_{m_{i}}\right)$. Usually, the leading $v$ power becomes more and more negative when $Q_{\tau}$ order $n$ goes up. When $n$ is enough high, we

\footnotetext{
${ }^{50}$ We also suppress the poles $\epsilon_{1}=\epsilon_{2}=0$, which are guaranteed by the $D_{\left\{d_{0}, d_{1}, d_{2}\right\}}^{\alpha^{\vee}} / D_{d}$ structure similar to the previous discussion.
} 
observe some patterns for the leading $v$-expansion behavior. For two-strings elliptic genus, we observe for $i \geq 2(\mathfrak{n}-2)$,

$$
g_{i, G}^{(2)}\left(v, x, Q_{m_{i}}\right)=-v^{-3(\mathfrak{n}+i)-5}\left(\chi_{(i-2(\mathfrak{n}-2)) \theta} \chi_{i-(\mathfrak{n}-2)}\right)-\mathcal{O}\left(v^{-3(\mathfrak{n}+i)-4}\right) \ldots
$$

As high as the $Q_{\tau}$ order we have reached, this is true. It is nice to see how to explain this phenomenon.

$\boldsymbol{A}_{\mathbf{2}}$. For the reduced two-string elliptic genus for $G=\mathrm{SU}(3)$ model, recall

$$
g_{2, A_{2}}^{(n)}\left(v, x=1, Q_{m_{i}}=1\right)=\frac{1}{(1-v)^{10}(1+v)^{6}\left(1+v+v^{2}\right)^{5}} \times P_{2, A_{2}}^{(n)}(v) .
$$

We have

$$
\begin{aligned}
& P_{2, A_{2}}^{(2)}(v)=v^{-10}\left(-1-v+6 v^{2}+9 v^{3}-10 v^{4}-33 v^{5}+41 v^{6}+256 v^{7}+428 v^{8}+220 v^{9}-347 v^{10}-823 v^{11}-131 v^{12}\right. \\
& \left.+2652 v^{13}+7721 v^{14}+14419 v^{15}+21826 v^{16}+27125 v^{17}+28966 v^{18}+27125 v^{19}+\cdots+v^{36}\right), \\
& P_{2, A_{2}}^{(3)}(v)=v^{-13}\left(-16-28 v+50 v^{2}+198 v^{3}+138 v^{4}-399 v^{5}-963 v^{6}-419 v^{7}+1716 v^{8}+4316 v^{9}+5014 v^{10}\right. \\
& +2174 v^{11}-4110 v^{12}-10701 v^{13}-12583 v^{14}-2128 v^{15}+27073 v^{16}+75426 v^{17}+136089 v^{18}+198723 v^{19} \\
& \left.+244336 v^{20}+260628 v^{21}+244336 v^{22}+\cdots+v^{42}\right), \\
& P_{2, A_{2}}^{(4)}(v)=v^{-16}\left(-81-223 v-5 v^{2}+880 v^{3}+1695 v^{4}+577 v^{5}-3110 v^{6}-6735 v^{7}-5803 v^{8}+2582 v^{9}+17019 v^{10}\right. \\
& +31735 v^{11}+38096 v^{12}+25783 v^{13}-11462 v^{14}-68637 v^{15}-118109 v^{16}-116300 v^{17}-11102 v^{18} \\
& \left.+231810 v^{19}+605425 v^{20}+1054375 v^{21}+1497688 v^{22}+1816145 v^{23}+1930514 v^{24}+\cdots+v^{48}\right), \\
& P_{2, A_{2}}^{(5)}(v)=v^{-19}\left(-256-874 v-810 v^{2}+1432 v^{3}+5510 v^{4}+7553 v^{5}+2483 v^{6}-10671 v^{7}-26644 v^{8}-34874 v^{9}\right. \\
& -23600 v^{10}+16620 v^{11}+90316 v^{12}+185964 v^{13}+260250 v^{14}+241846 v^{15}+62988 v^{16}-279728 v^{17} \\
& -692866 v^{18}-963287 v^{19}-815649 v^{20}+30457 v^{21}+1729760 v^{22}+4189517 v^{23}+7044615 v^{24}+9777494 v^{25} \\
& \left.+11727151 v^{26}+12428414 v^{27}+11727151 v^{28}+\cdots+v^{54}\right), \\
& \text {... } \\
& P_{2, A_{2}}^{(10)}(v)(v)=v^{-34}\left(-6561-31129 v-72335 v^{2}-120018 v^{3}-168868 v^{4}-206886 v^{5}-222250 v^{6}-170936 v^{7}+98441 v^{8}\right. \\
& +773013 v^{9}+1959712 v^{10}+3612563 v^{11}+5410138 v^{12}+6633691 v^{13}+5994478 v^{14}+1510639 v^{15} \\
& -9064153 v^{16}-27314880 v^{17}-52091127 v^{18}-77137569 v^{19}-89646012 v^{20}-69861741 v^{21}+5587892 v^{22} \\
& +154737705 v^{23}+377669387 v^{24}+641219584 v^{25}+868425362 v^{26}+940214412 v^{27}+718317837 v^{28} \\
& +93293293 v^{29}-951170969 v^{30}-2275980208 v^{31}-3545719630 v^{32}-4250592736 v^{33}-3785597548 v^{34} \\
& -1612094548 v^{35}+2569710910 v^{36}+8659446500 v^{37}+16075828305 v^{38}+23808502207 v^{39} \\
& \left.+30625221095 v^{40}+35302136340 v^{41}+36965592032 v^{42}+35302136340 v^{43}+\cdots+v^{84}\right) .
\end{aligned}
$$

Note that the leading power of $v$ in $P_{2, A_{2}}^{(n)}(v)$ becomes more and more negative as $n$ increases. In fact, we notice for $n \geq 2$,

$$
P_{2, A_{2}}^{(n)}(v)=v^{-3 n-4}\left(-(n-1)^{4}-\cdots+\text { palindrome up to } v^{6(n+4)}\right) .
$$

With generic $x$, we also observe the following general expansion: for $n \geq 2$,

$$
v^{3 n+4} g_{2, A_{2}}^{(n)}\left(v, x, Q_{m_{i}}\right)=-\chi_{(n-2) \theta}^{A_{2}} \chi_{n-1}-\left(\chi_{(n-3) \theta}^{A_{2}} \chi_{n}+\ldots\right) v-\left(\chi_{(n-4) \theta}^{A_{2}} \chi_{n+1}+\ldots\right) v^{2}+\ldots
$$


For the reduced elliptic genus of three-strings, recall

$$
g_{3, A_{2}}^{(n)}\left(v, x=1, Q_{m_{i}}=1\right)=\frac{1}{(1-v)^{16}(1+v)^{10}\left(1+v^{2}\right)^{5}\left(1+v+v^{2}\right)^{6}} \times P_{3, A_{2}}^{(n)}(v) .
$$

We obtain

$$
\begin{aligned}
& P_{3, A_{2}}^{(2)}(v)=v^{-10}\left(-1-2 v-5 v^{2}-4 v^{3}+11 v^{4}+30 v^{5}+74 v^{6}+84 v^{7}+267 v^{8}+880 v^{9}+2718 v^{10}+6130 v^{11}\right. \\
& +11512 v^{12}+17594 v^{13}+24774 v^{14}+35306 v^{15}+64404 v^{16}+133960 v^{17}+290609 v^{18}+569846 v^{19} \\
& +1020364 v^{20}+1628376 v^{21}+2376984 v^{22}+3145582 v^{23}+3848955 v^{24}+4318298 v^{25}+4501676 v^{26} \\
& \left.+4318298 v^{27}+\cdots+v^{52}\right) \text {, } \\
& P_{3, A_{2}}^{(3)}(v)=v^{-17}\left(2-10 v^{2}-9 v^{3}+12 v^{4}+44 v^{5}-48 v^{6}-367 v^{7}-622 v^{8}-183 v^{9}+1740 v^{10}+4336 v^{11}+4488 v^{12}\right. \\
& -1116 v^{13}-12504 v^{14}-17424 v^{15}+4576 v^{16}+77007 v^{17}+199630 v^{18}+337366 v^{19}+417362 v^{20} \\
& +382080 v^{21}+255946 v^{22}+265962 v^{23}+839446 v^{24}+2662413 v^{25}+6368124 v^{26}+12524957 v^{27} \\
& +21034734 v^{28}+31405824 v^{29}+42159674 v^{30}+51803451 v^{31}+58351834 v^{32}+60785174 v^{33} \\
& \left.+58351834 v^{34}+\ldots+v^{66}\right) \text {, } \\
& P_{3, A_{2}}^{(4)}(v)=v^{-21}\left(32+40 v-84 v^{2}-339 v^{3}-426 v^{4}+487 v^{5}+2242 v^{6}+2876 v^{7}-1500 v^{8}-12817 v^{9}-23440 v^{10}\right. \\
& -16831 v^{11}+25630 v^{12}+97493 v^{13}+150494 v^{14}+103012 v^{15}-112582 v^{16}-448583 v^{17}-700938 v^{18} \\
& -495362 v^{19}+512476 v^{20}+2411835 v^{21}+4741886 v^{22}+6461123 v^{23}+6254586 v^{24}+3310035 v^{25} \\
& -1672684 v^{26}-5447913 v^{27}-2195270 v^{28}+16177917 v^{29}+57171170 v^{30}+126228382 v^{31}+222042686 v^{32} \\
& \left.+337620393 v^{33}+457167512 v^{34}+563129124 v^{35}+635332178 v^{36}+661625872 v^{37}+635332178 v^{38}+\cdots+v^{74}\right), \\
& P_{3, A_{2}}^{(5)}(v)=v^{-25}\left(162+412 v+276 v^{2}-1295 v^{3}-4518 v^{4}-5771 v^{5}+1292 v^{6}+21793 v^{7}+44712 v^{8}+38030 v^{9}\right. \\
& -38688 v^{10}-192767 v^{11}-341004 v^{12}-309898 v^{13}+101910 v^{14}+927411 v^{15}+1862626 v^{16}+2189163 v^{17} \\
& +1001340 v^{18}-2210183 v^{19}-6887108 v^{20}-10727974 v^{21}-10030208 v^{22}-703145 v^{23}+19133352 v^{24} \\
& +46719280 v^{25}+72876216 v^{26}+83251991 v^{27}+63444248 v^{28}+7529926 v^{29}-72005488 v^{30}-136950773 v^{31} \\
& -124639134 v^{32}+43752233 v^{33}+438669558 v^{34}+1103016208 v^{35}+2019390168 v^{36}+3111882801 v^{37} \\
& \left.+4234930428 v^{38}+5220665928 v^{39}+5892000612 v^{40}+6133530828 v^{41}+5892000612 v^{42}+\cdots+v^{82}\right), \\
& P_{3, A_{2}}^{(6)}(v)=v^{-29}\left(512+1690 v+3044 v^{2}+766 v^{3}-10596 v^{4}-31191 v^{5}-46930 v^{6}-19288 v^{7}+89188 v^{8}+274511 v^{9}\right. \\
& +419176 v^{10}+288140 v^{11}-374722 v^{12}-1614464 v^{13}-2963132 v^{14}-3347473 v^{15}-1280918 v^{16} \\
& +4247141 v^{17}+12688484 v^{18}+20686636 v^{19}+22001562 v^{20}+9184210 v^{21}-22497646 v^{22}-69062433 v^{23} \\
& -113553734 v^{24}-124631077 v^{25}-65395112 v^{26}+91413273 v^{27}+339731228 v^{28}+622477442 v^{29} \\
& +828007584 v^{30}+813952151 v^{31}+463211226 v^{32}-241280313 v^{33}-1140297436 v^{34}-1857927117 v^{35} \\
& -1829759686 v^{36}-398271211 v^{37}+2994640544 v^{38}+8636915664 v^{39}+16332876522 v^{40}+25397471432 v^{41} \\
& \left.+34642293136 v^{42}+42690290417 v^{43}+48157593258 v^{44}+50110832268 v^{45}+48157593258 v^{46}+\cdots+v^{90}\right) \text {. }
\end{aligned}
$$

For $n \geq 3$, we notice the following universal leading behavior

$$
g_{n, A_{2}}^{(3)}(v, x)=v^{-4 n-5}\left(\chi_{2(n-2)} \chi_{(n-3) \theta}+\mathcal{O}(v)\right) .
$$

$\boldsymbol{D}_{\mathbf{4}}$. For the reduced two-string elliptic genus of SO(8) 6d SCFT, recall

$$
g_{2, D_{4}}^{(n)}\left(v, x=1, Q_{m_{i}}=1\right)=\frac{1}{(1-v)^{22}(1+v)^{12}\left(1+v+v^{2}\right)^{11}} \times P_{2, D_{4}}^{(n)}(v) .
$$


We have

$$
\begin{aligned}
& P_{2, D_{4}}^{(2)}(v)=-v^{-8}\left(1+3 v+22 v^{2}+47 v^{3}+108 v^{4}+29 v^{5}-184 v^{6}-861 v^{7}-1762 v^{8}-3305 v^{9}-13965 v^{10}-65210 v^{11}\right. \\
& -260932 v^{12}-884324 v^{13}-2589008 v^{14}-6645978 v^{15}-15280924 v^{16}-31673046 v^{17}-59506626 v^{18} \\
& -101871752 v^{19}-159566189 v^{20}-229273231 v^{21}-303095099 v^{22}-369460130 v^{23}-415787266 v^{24} \\
& \left.-432409780 v^{25}-415787266 v^{26}+\cdots+v^{50}\right) \text {, } \\
& P_{2, D_{4}}^{(3)}(v)=v^{-11}\left(1+v^{2}\right)\left(2+3 v-23 v^{2}-281 v^{3}-1338 v^{4}-3786 v^{5}-5794 v^{6}-384 v^{7}+22410 v^{8}+57106 v^{9}\right. \\
& +65200 v^{10}-8878 v^{11}-148110 v^{12}-82859 v^{13}+911821 v^{14}+4473554 v^{15}+14488449 v^{16}+39847612 v^{17} \\
& +97908991 v^{18}+217119419 v^{19}+434067674 v^{20}+782158341 v^{21}+1274332193 v^{22}+1885916954 v^{23} \\
& \left.+2545465687 v^{24}+3144719815 v^{25}+3566407238 v^{26}+3718703248 v^{27}+3566407238 v^{28}+\cdots+v^{54}\right), \\
& P_{2, D_{4}}^{(4)}(v)=-v^{-19}\left(2+2 v-22 v^{2}-42 v^{3}+88 v^{4}+329 v^{5}-11 v^{6}-1411 v^{7}-1930 v^{8}+1877 v^{9}+12149 v^{10}\right. \\
& +38843 v^{11}+113904 v^{12}+240220 v^{13}+226932 v^{14}-364906 v^{15}-1770622 v^{16}-3067485 v^{17}-1974439 v^{18} \\
& +3262797 v^{19}+10272490 v^{20}+10304550 v^{21}-10767498 v^{22}-74227039 v^{23}-228511951 v^{24} \\
& -601571798 v^{25}-1479273049 v^{26}-3376185023 v^{27}-7028420238 v^{28}-13251946148 v^{29} \\
& -22656311766 v^{30}-35281600040 v^{31}-50298462814 v^{32}-65951082224 v^{33}-79826800694 v^{34} \\
& \left.-89424037262 v^{35}-92856436862 v^{36}-89424037262 v^{37}+\cdots+v^{72}\right) \text {, } \\
& P_{2, D_{4}}^{(5)}(v)=-v^{-22}\left(1+v^{2}\right)\left(84+146 v-831 v^{2}-2495 v^{3}+2027 v^{4}+16874 v^{5}+12844 v^{6}-55230 v^{7}-118023 v^{8}\right. \\
& +45251 v^{9}+412658 v^{10}+306961 v^{11}-747606 v^{12}-1085075 v^{13}+2514527 v^{14}+8955862 v^{15}+7677132 v^{16} \\
& -13638464 v^{17}-48153754 v^{18}-58843606 v^{19}-3166625 v^{20}+114419603 v^{21}+211444789 v^{22} \\
& +164548302 v^{23}-98377407 v^{24}-542911427 v^{25}-1148224939 v^{26}-2306012154 v^{27}-5445277331 v^{28} \\
& -13585439053 v^{29}-31372682848 v^{30}-64179900027 v^{31}-116221363135 v^{32}-188200853472 v^{33} \\
& -275432340276 v^{34}-367411722852 v^{35}-449506711376 v^{36}-506517773112 v^{37}-526950799964 v^{38} \\
& \left.-506517773112 v^{39}+\cdots+v^{76}\right), \\
& P_{2, D_{4}}^{(6)}(v)=-v^{-25}\left(1200+3374 v-6724 v^{2}-40296 v^{3}-31436 v^{4}+159328 v^{5}+410682 v^{6}+15592 v^{7}-1467224 v^{8}\right. \\
& -2279805 v^{9}+1088019 v^{10}+8007211 v^{11}+8314028 v^{12}-9090084 v^{13}-32918254 v^{14}-19726713 v^{15} \\
& +67719233 v^{16}+186559606 v^{17}+183703487 v^{18}-107579194 v^{19}-661966959 v^{20}-1107115384 v^{21} \\
& -832956121 v^{22}+557841530 v^{23}+2672286699 v^{24}+4109758618 v^{25}+3046056345 v^{26}-1483241349 v^{27} \\
& -8623040702 v^{28}-16826056321 v^{29}-28634625983 v^{30}-59027415291 v^{31}-144463390006 v^{32} \\
& -347331281609 v^{33}-749797256633 v^{34}-1433909622002 v^{35}-2450540661913 v^{36}-3786292890317 v^{37} \\
& -5341633935638 v^{38}-6932828077068 v^{39}-8322655072044 v^{40}-9274316155458 v^{41}-9612992092064 v^{42} \\
& \left.-9274316155458 v^{43}+\cdots+v^{84}\right) \text {. }
\end{aligned}
$$

For $n \geq 4$, we observe the following general leading order behavior

$$
v^{3 n+7} g_{2, D_{4}}^{(n)}\left(v, x, Q_{m_{i}}\right)=-\chi_{(n-4) \theta}^{D_{4}} \chi_{n-2}(x)+\mathcal{O}(v) .
$$

$\boldsymbol{F}_{4}$. For the reduced two-string elliptic genus of $F_{4} 6 \mathrm{~d}$ SCFT, recall

$$
g_{2, F_{4}}^{(n)}\left(v, x=1, Q_{m_{i}}=1\right)=\frac{1}{(1-v)^{34}(1+v)^{22}\left(1+v+v^{2}\right)^{17}} \times P_{2, F_{4}}^{(n)}(v) .
$$


We have

$$
\begin{aligned}
& P_{2, F_{4}}^{(2)}(v)=1653+14307 v+118305 v^{2}+770928 v^{3}+4293754 v^{4}+20938534 v^{5}+90874761 v^{6}+354378137 v^{7} \\
& +1254181600 v^{8}+4057153817 v^{9}+12068000241 v^{10}+33173318084 v^{11}+84638789902 v^{12}+201171570880 v^{13} \\
& +446852450528 v^{14}+930177353330 v^{15}+1818950197662 v^{16}+3348446417048 v^{17}+5813458948881 v^{18} \\
& +9534395062259 v^{19}+14791974953829 v^{20}+21734938030680 v^{21}+30278882934513 v^{22}+40026745086453 v^{23} \\
& +50246339190488 v^{24}+59931545438647 v^{25}+67951300040637 v^{26}+73260270949890 v^{27} \\
& +75118982210308 v^{28}+73260270949890 v^{29}+\cdots+v^{56}, \\
& P_{2, F_{4}}^{(3)}(v)=v^{-12}\left(1+7 v+58 v^{2}+277 v^{3}+1071 v^{4}+2976 v^{5}+5964 v^{6}+5832 v^{7}-9266 v^{8}-57418 v^{9}-135552 v^{10}\right. \\
& -169110 v^{11}+55432 v^{12}+957484 v^{13}+4373263 v^{14}+21374265 v^{15}+111764209 v^{16}+546538072 v^{17} \\
& +2392703794 v^{18}+9374157248 v^{19}+33193123730 v^{20}+107176864396 v^{21}+317784470985 v^{22} \\
& +870042414425 v^{23}+2209544249477 v^{24}+5224968453408 v^{25}+11543089455336 v^{26}+23893104753132 v^{27} \\
& +46455227531026 v^{28}+85029824340612 v^{29}+146800695692191 v^{30}+239466759024769 v^{31} \\
& +369631201685833 v^{32}+540578567158590 v^{33}+749891181508407 v^{34}+987634558444181 v^{35} \\
& +1235929372818009 v^{36}+1470501793161916 v^{37}+1664270696390466 v^{38}+1792334859311106 v^{39} \\
& \left.+1837134386523548 v^{40}+1792334859311106 v^{41}+\cdots+v^{80}\right), \\
& P_{2, F_{4}}^{(4)}(v)=-v^{-15}\left(2+11 v-9 v^{2}-729 v^{3}-6740 v^{4}-37612 v^{5}-146242 v^{6}-411322 v^{7}-786424 v^{8}-683123 v^{9}\right. \\
& +1519991 v^{10}+7863641 v^{11}+17299644 v^{12}+19396808 v^{13}-7574716 v^{14}-81039308 v^{15}-177701238 v^{16} \\
& -228893241 v^{17}-328644969 v^{18}-1704624732 v^{19}-10239254139 v^{20}-48437902734 v^{21}-193165328741 v^{22} \\
& -683657580163 v^{23}-2198459381202 v^{24}-6491341536008 v^{25}-17700027945928 v^{26}-44757889196479 v^{27} \\
& -105345430184255 v^{28}-231557373968430 v^{29}-476759190688969 v^{30}-921916213048446 v^{31} \\
& -1678228390978831 v^{32}-2881832101851776 v^{33}-4676542813241321 v^{34}-7182956156002759 v^{35} \\
& -10456822688468996 v^{36}-14445439874041454 v^{37}-18955600691329352 v^{38}-23647746652092629 v^{39} \\
& -28066464498494385 v^{40}-31707630617443838 v^{41}-34110133094328607 v^{42}-34949875241183086 v^{43} \\
& \left.-34110133094328607 v^{44}+\cdots+v^{86}\right) \text {, } \\
& P_{2, F_{4}}^{(5)}(v)=v^{-18}\left(1+v-187 v^{2}-1942 v^{3}-8588 v^{4}+388 v^{5}+256583 v^{6}+1875127 v^{7}+8097680 v^{8}+23995511 v^{9}\right. \\
& +47743204 v^{10}+45403031 v^{11}-77639509 v^{12}-441768092 v^{13}-990656365 v^{14}-1141697391 v^{15} \\
& +314912356 v^{16}+4322862537 v^{17}+9413229443 v^{18}+9932344114 v^{19}-1369097445 v^{20}-22028614537 v^{21} \\
& -15689824140 v^{22}+143994570705 v^{23}+853983234794 v^{24}+3391440788051 v^{25}+11730377038184 v^{26} \\
& +37232002426385 v^{27}+109311773773871 v^{28}+297084435895112 v^{29}+748700764659463 v^{30} \\
& +1754971776388633 v^{31}+3839341467548316 v^{32}+7864675977003847 v^{33}+15128265661342010 v^{34} \\
& +27394204368470677 v^{35}+46797420093685421 v^{36}+75560202097716654 v^{37}+115501696318370322 v^{38} \\
& +167393226212698984 v^{39}+230299469534789175 v^{40}+301112810847585121 v^{41}+374497086121044331 v^{42} \\
& +443382800033202476 v^{43}+500006416441373208 v^{44}+537304885313273980 v^{45}+550330680323572096 v^{46} \\
& \left.+537304885313273980 v^{47}+\cdots+v^{92}\right) .
\end{aligned}
$$

For $n \geq 6$, we observe the following general leading order behavior

$$
v^{3 n+10} g_{2, F_{4}}^{(n)}\left(v, x, Q_{m_{i}}\right)=-\chi_{(n-6) \theta}^{F_{4}} \chi_{n-3}(x)+\mathcal{O}(v) .
$$

$\boldsymbol{E}_{6}$. For the reduced two-string elliptic genus of $E_{6} 6 \mathrm{~d}$ SCFT, recall

$$
g_{2, E_{6}}^{(n)}\left(v, x, Q_{m_{i}}=1\right)=\frac{1}{(1-v)^{46}(1+v)^{32}\left(1+v+v^{2}\right)^{23}} \times P_{2, E_{6}}^{(n)}(v) .
$$


We have

$$
\begin{aligned}
P_{2, E_{6}}^{(1)}(v) & =\left(1+v^{2}\right)\left(82+896 v+9129 v^{2}+73825 v^{3}+515477 v^{4}+3176394 v^{5}+17567385 v^{6}+88082527 v^{7}\right. \\
& +404122599 v^{8}+1707996910 v^{9}+6687039606 v^{10}+24365673656 v^{11}+82957003626 v^{12} \\
& +264812209428 v^{13}+794925309293 v^{14}+2249848989493 v^{15}+6017588149603 v^{16}+15241390482586 v^{17} \\
& +36623148751459 v^{18}+83623554563863 v^{19}+181712020504595 v^{20}+376267731853770 v^{21} \\
& +743340720549339 v^{22}+1402570753853399 v^{23}+2530053857442778 v^{24}+4367001323365453 v^{25} \\
& +7218179887542376 v^{26}+11433257908228549 v^{27}+17365401325615558 v^{28}+25305594210396759 v^{29} \\
& +35398201343930359 v^{30}+47551931562200552 v^{31}+61367940071565626 v^{32} \\
& +76109936363599780 v^{33}+90737018750916024 v^{34}+104007721490984500 v^{35} \\
& \left.+114645634265369518 v^{36}+121537998101131452 v^{37}+123925354694394472 v^{38}+\cdots+v^{76}\right) . \\
P_{2, E_{6}}^{(2)}(v) & =3486+44488 v+464913 v^{2}+3873323 v^{3}+27606333 v^{4}+172731506 v^{5}+966630727 v^{6}+4894721995 v^{7} \\
& +22642609833 v^{8}+96385144324 v^{9}+379809898432 v^{10}+1392335683050 v^{11}+4768406721146 v^{12} \\
& +15311706805952 v^{13}+46244599549903 v^{14}+131729726893973 v^{15}+354773291170080 v^{16} \\
& +905322588772629 v^{17}+2193217132886674 v^{18}+5052883393549785 v^{19}+11088020153427871 v^{20} \\
& +23207949295452654 v^{21}+46391596457503471 v^{22}+88666687652950697 v^{23}+162200225646883046 v^{24} \\
& +284262935556020849 v^{25}+477678248920949928 v^{26}+770246305708025175 v^{27} \\
& +1192619227946678339 v^{28}+1774271549538256254 v^{29}+2537602212267590587 v^{30} \\
& +3490792045588793343 v^{31}+4620696016056616197 v^{32}+5887558970641741644 v^{33} \\
& +7223464858978994614 v^{34}+8535984817703595260 v^{35}+9717463741648588196 v^{36} \\
& +10658977913348459838 v^{37}+11266596547576742504 v^{38}+11476648364287371362 v^{39} \\
& +11266596547576742504 v^{40}+\cdots+v^{78} .
\end{aligned}
$$

$$
\begin{aligned}
P_{2, E_{6}}^{(3)}(v) & =v^{-4}\left(-1-11 v-112 v^{2}-769 v^{3}+97959 v^{4}+1465146 v^{5}+15949836 v^{6}+136827854 v^{7}+992270036 v^{8}\right. \\
& +6276463714 v^{9}+35347238184 v^{10}+179602878296 v^{11}+831787432544 v^{12}+3538777989264 v^{13} \\
& +13918341585911 v^{14}+50873087148945 v^{15}+173571288630315 v^{16}+554884515982156 v^{17} \\
& +1667591219224745 v^{18}+4724849885190467 v^{19}+12653182331604747 v^{20}+32099832942977106 v^{21} \\
& +77298235563217848 v^{22}+177003542946746952 v^{23}+386049290828201197 v^{24} \\
& +803129445851193549 v^{25}+1595815215747637914 v^{26}+3032155653955325045 v^{27} \\
& +5515192004170661557 v^{28}+9612446692952872376 v^{29}+16067738341715188425 v^{30} \\
& +25779182779041415519 v^{31}+39727277739048609244 v^{32}+58842809938209124305 v^{33} \\
& +83817103245449704330 v^{34}+114875661886259702901 v^{35}+151556399981844604507 v^{36} \\
& +192548720900049944088 v^{37}+235652392733234663776 v^{38}+277900332002367574858 v^{39} \\
& +315856987228959670753 v^{40}+346060446910493617699 v^{41}+365533661213463473501 v^{42} \\
& \left.+372262200765577638648 v^{43}+\cdots+v^{86}\right) . \\
P_{2, E_{6}}^{(4)}(v) & =v^{-16}\left(-1-11 v-112 v^{2}-769 v^{3}-4214 v^{4}-18313 v^{5}-64197 v^{6}-177594 v^{7}-364431 v^{8}-421607 v^{9}\right. \\
& +420751 v^{10}+3722444 v^{11}+10737460 v^{12}+18191652 v^{13}+11206753 v^{14}-38487665 v^{15} \\
& +147892027 v^{16}-235567050 v^{17}+204232919 v^{18}+4014329887 v^{19}+28747116555 v^{20} \\
& +177469181418 v^{21}+994624050267 v^{22}+5061754204737 v^{23}+23475175955326 v^{24} \\
& +99852192764195 v^{25}+392058843196059 v^{26}+1428966813600884 v^{27}+4857570355921361 v^{28} \\
& +15462381593811917 v^{29}+46247090390273044 v^{30}+130358984643118795 v^{31} \\
& +347206472133377093 v^{32}+875864441137943176 v^{33}+2096960972906450647 v^{34}
\end{aligned}
$$




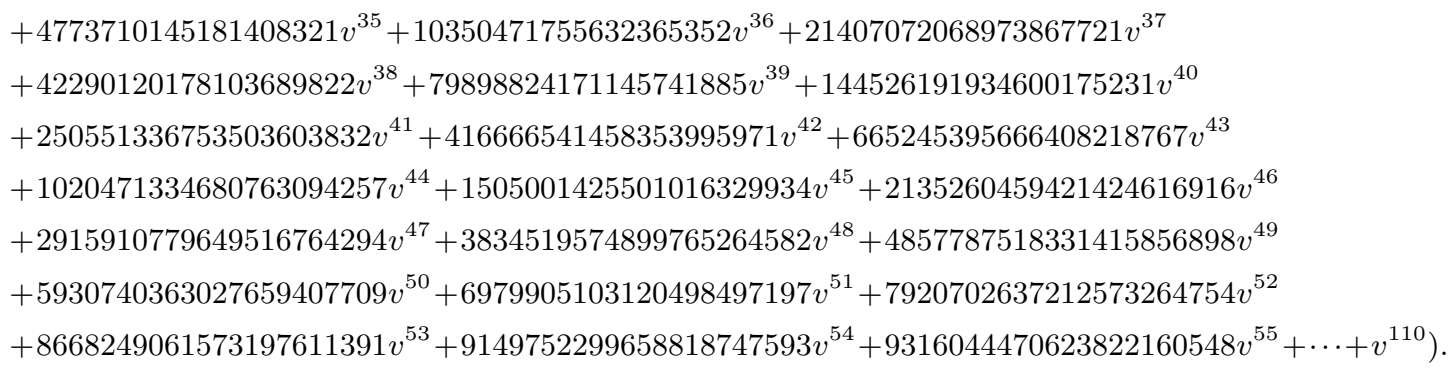

$\boldsymbol{E}_{7}$. For the reduced two-string elliptic genus of $E_{7} 6$ d SCFT, recall

$$
g_{2, E_{7}}^{(n)}\left(v, x, Q_{m_{i}}=1\right)=\frac{1}{(1-v)^{70}(1+v)^{52}\left(1+v+v^{2}\right)^{35}} \times P_{n, E_{7}}^{(2)}(v) .
$$

We have

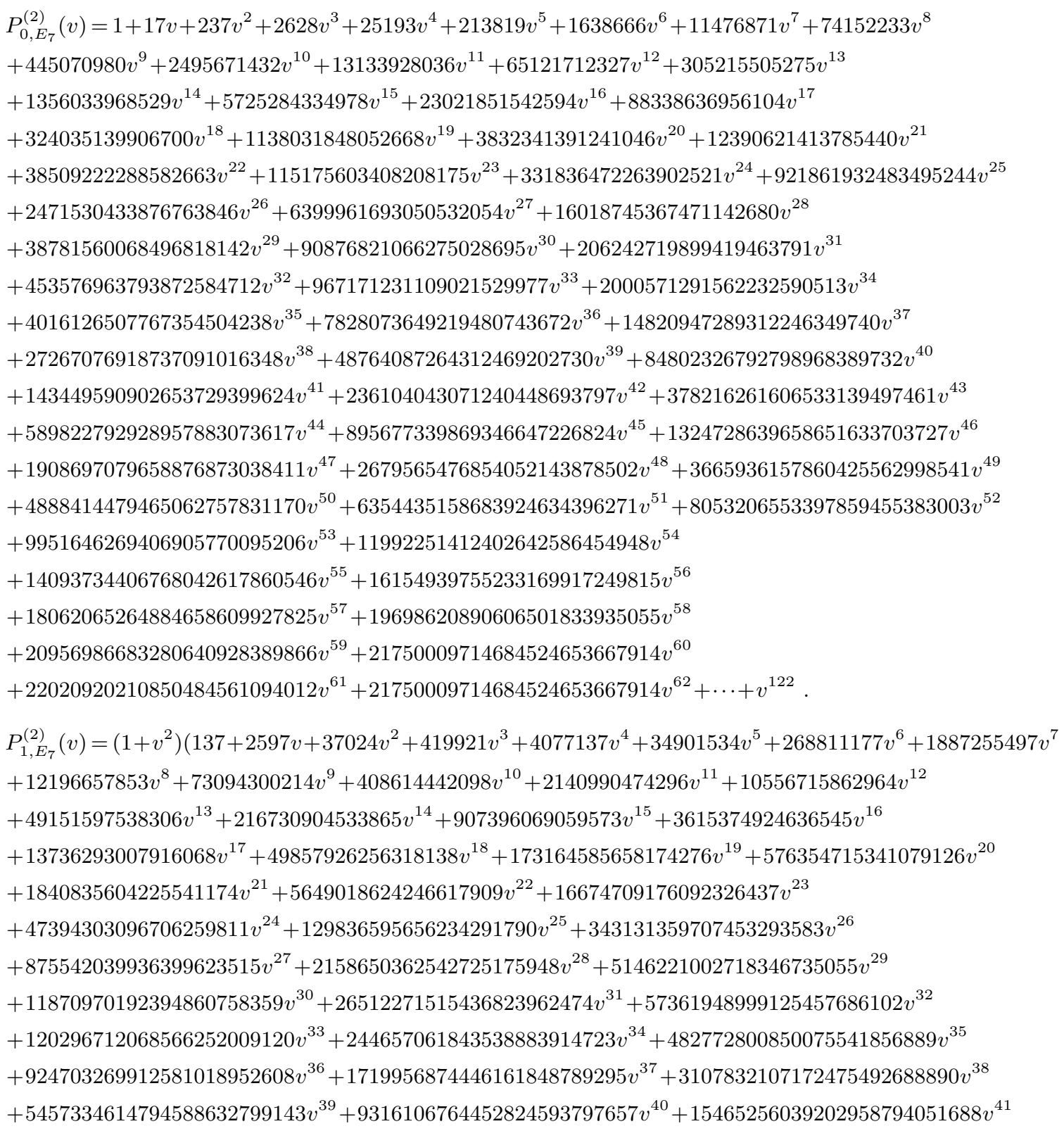




$$
\begin{aligned}
& +24973370386295921380753761 v^{42}+39238673033178891558314265 v^{43}+60003949644181883287996554 v^{44} \\
& +89325800153382434388949763 v^{45}+129479490500199449940430040 v^{46}+182784785431420765743008945 v^{47} \\
& +251347720581234682951528991 v^{48}+336728327510605097378060508 v^{49}+439563294320255738140535927 v^{50} \\
& +559192119429259737303598283 v^{51}+693350791574808124298361559 v^{52}+838003231433873171234645238 v^{53} \\
& +987373409206497489976018270 v^{54}+1134218396741161783456978908 v^{55} \\
& +1270346407634715071774123344 v^{56}+1387339824356009883032251758 v^{57} \\
& +1477400106011059794784293700 v^{58}+1534199301083129786878770830 v^{59} \\
& \left.+1553610262702054425407310320 v^{60}+\cdots+v^{120}\right) .
\end{aligned}
$$

Note $g_{0, E_{7}}^{(2)}$ agree with the two-instanton $E_{7}$ Hilbert series in [42].

\section{G Refined BPS invariants}

The refined BPS invariants are solved from the generalised blowup equations with the following initial input: the triple intersection numbers $\kappa_{i j k}$ of divisors, the intersection numbers $b_{i}^{\mathrm{GV}}$ of divisors with $c_{2}(X)$ (these are two ingredients of $Z^{\mathrm{cls}}$ ), the curve-divisor intersection matrix $C$, one unity $r$ field with nonzero $r_{b}$, as well as the one-loop partition function $Z^{1 \text {-loop }}$ and the bounds $j_{l, r}^{\max }$.

In the case of $\mathfrak{n}=5,12$ models, there is no vanishing $r$-fields, the unity $r$ do not have enough constraints on $Z^{1 \text {-loop }}$. On the other hand, as seen in (2.36), $Z^{1 \text {-loop }}$ is easily computed, we simply input $Z^{1 \text {-loop }}$ for all the models.

The input of $j_{l, r}^{\max }$ is strictly speaking also not necessary, as the bounds can be generated from the blowup equations with the other input data, but the inclusion of the bounds in the program makes the computation much faster. In any case, for $d_{b} \geq 1$, we observe an experimental formula for $j_{l, r}^{\max }$. For $F_{4}$, we observed for $d+d_{b} \leq 14$

$$
\begin{aligned}
& j_{r}^{\max }\left(d, d_{b}\right)=d\left(d_{b}+1\right)-d_{b}\left(d_{b}-1\right) / 2, \\
& j_{l}^{\max }\left(d, d_{b}\right)=(d-1)\left(d_{b}-1\right)-d_{b}\left(d_{b}-1\right) / 2+\delta_{d_{b}, 1}\left\lfloor d /\left(1+d_{\tau}\right)\right\rfloor,
\end{aligned}
$$

where $d$ is the total degree of the fibers, $d_{b}$ is the degree of the base, and $d_{\tau}$ is the first total degree of fibers when $\tau$ appears. For $E_{6,7,8}$, we observed for $d+d_{b} \leq 12 \leq d_{\tau}$

$$
\begin{aligned}
& j_{r}^{\max }\left(d, d_{b}\right)=d_{b}+d\left(d_{b}+1\right), \\
& j_{l}^{\max }\left(d, d_{b}\right)=(d-1)\left(d_{b}-1\right) .
\end{aligned}
$$

For $F_{4}$ model, we compute all the refined BPS invariants up to total degree 14, with 4777 non-vanishing. For $E_{6,7,8}$, we compute all the BPS invariants up to total degree 12 , with 10383, 10491, 10068 non-vanishing respectively. We list part of the refined BPS invariants in the affine Lie algebra bases in tables 24-31, for complete lists, one can find them at [31]. It is worthwhile to point out that unlike the elliptic genus [24], the Weyl symmetry of gauge group $G$ is not manifest in the refined BPS invariants. This is simply because the Weyl symmetry will change the sign of some Kähler parameters, while the refined BPS expansion is always in positive degrees. Note this should not be confused with the situation where the refined BPS invariants of E-strings do have manifest $E_{8}$ symmetry, in which case the $E_{8}$ is a global symmetry other than the gauge symmetry $G$ we considered in this paper. 


\begin{tabular}{|c|c|c|c|}
\hline$\beta$ & $\oplus N_{j_{l}, j_{r}}^{\beta}\left(j_{l}, j_{r}\right)$ & $\beta$ & $\oplus N_{j_{l}, j_{r}}^{\beta}\left(j_{l}, j_{r}\right)$ \\
\hline$(0,0,0,0,0,1)$ & $(0,0)$ & $(0,0,1,0,0,1)$ & $(0,1)$ \\
\hline$(0,0,1,1,0,1)$ & $(0,0) \oplus(0,1)$ & $(0,0,1,1,1,1)$ & $(0,0) \oplus(0,1)$ \\
\hline$(0,0,1,2,0,1)$ & $(0,1)$ & \begin{tabular}{|l|l|}
$(0,0,1,2,1,1)$ \\
\end{tabular} & $(0,0) \oplus(0,1)$ \\
\hline$(0,0,1,2,2,1)$ & $(0,1)$ & $(0,0,2,0,0,1)$ & $(0,2)$ \\
\hline$(0,0,2,1,0,1)$ & $(0,1) \oplus(0,2)$ & $(0,0,2,1,1,1)$ & $(0,1) \oplus(0,2)$ \\
\hline$(0,0,2,2,0,1)$ & $(0,0) \oplus(0,1) \oplus(0,2)$ & $(0,0,2,2,1,1)$ & $(0,0) \oplus 2(0,1) \oplus(0,2)$ \\
\hline$(0,0,2,3,0,1)$ & $(0,1) \oplus(0,2)$ & $(0,0,3,0,0,1)$ & $(0,3)$ \\
\hline$(0,0,3,1,0,1)$ & $(0,2) \oplus(0,3)$ & $(0,0,3,1,1,1)$ & $(0,2) \oplus(0,3)$ \\
\hline$(0,0,3,2,0,1)$ & $(0,1) \oplus(0,2) \oplus(0,3)$ & $(0,0,4,0,0,1)$ & $(0,4)$ \\
\hline$(0,0,4,1,0,1)$ & $(0,3) \oplus(0,4)$ & $(0,0,5,0,0,1)$ & $(0,5)$ \\
\hline$(0,1,0,0,0,1)$ & $(0,1)$ & $(0,1,1,0,0,1)$ & $(0,0) \oplus(0,1)$ \\
\hline$(0,1,1,1,0,1)$ & $(0,0) \oplus(0,1)$ & $(0,1,1,1,1,1)$ & $(0,0) \oplus(0,1)$ \\
\hline$(0,1,1,2,0,1)$ & $(0,0) \oplus(0,1)$ & $(0,1,1,2,1,1)$ & $(0,0) \oplus(0,1)$ \\
\hline$(0,1,2,0,0,1)$ & $(0,1) \oplus(0,2)$ & $(0,1,2,1,0,1)$ & $(0,0) \oplus 2(0,1) \oplus(0,2)$ \\
\hline$(0,1,2,1,1,1)$ & $(0,0) \oplus 2(0,1) \oplus(0,2)$ & $(0,1,2,2,0,1)$ & $2(0,0) \oplus 3(0,1) \oplus(0,2)$ \\
\hline$(0,1,3,0,0,1)$ & $(0,2) \oplus(0,3)$ & $(0,1,3,1,0,1)$ & $(0,1) \oplus 2(0,2) \oplus(0,3)$ \\
\hline$(0,1,4,0,0,1)$ & $(0,3) \oplus(0,4)$ & $(0,2,0,0,0,1)$ & $(0,2)$ \\
\hline$(0,2,1,0,0,1)$ & $(0,1) \oplus(0,2)$ & $(0,2,1,1,0,1)$ & $(0,1) \oplus(0,2)$ \\
\hline$(0,2,1,1,1,1)$ & $(0,1) \oplus(0,2)$ & (0,2,1,2,0,1) & $(0,1) \oplus(0,2)$ \\
\hline$(0,2,2,0,0,1)$ & $(0,0) \oplus(0,1) \oplus(0,2)$ & $(0,2,2,1,0,1)$ & $(0,0) \oplus 2(0,1) \oplus(0,2)$ \\
\hline$(0,2,3,0,0,1)$ & $(0,1) \oplus(0,2) \oplus(0,3)$ & $(0,3,0,0,0,1)$ & $(0,3)$ \\
\hline$(0,3,1,0,0,1)$ & $(0,2) \oplus(0,3)$ & $(0,3,1,1,0,1)$ & $(0,2) \oplus(0,3)$ \\
\hline$(0,3,2,0,0,1)$ & $(0,1) \oplus(0,2) \oplus(0,3)$ & $(0,4,0,0,0,1)$ & $(0,4)$ \\
\hline$(0,4,1,0,0,1)$ & $(0,3) \oplus(0,4)$ & $(0,5,0,0,0,1)$ & $(0,5)$ \\
\hline$(1,1,0,0,0,1)$ & $(0,0) \oplus(0,1)$ & $(1,1,1,0,0,1)$ & $(0,0) \oplus(0,1)$ \\
\hline$(1,1,1,1,0,1)$ & $(0,0) \oplus(0,1)$ & $(1,1,1,1,1,1)$ & $(0,0) \oplus(0,1)$ \\
\hline$(1,1,1,2,0,1)$ & $(0,0) \oplus(0,1)$ & $(1,1,2,0,0,1)$ & $(0,1) \oplus(0,2)$ \\
\hline$(1,1,2,1,0,1)$ & $(0,0) \oplus 2(0,1) \oplus(0,2)$ & $\mid(1,1,3,0,0,1)$ & $(0,2) \oplus(0,3)$ \\
\hline$(1,2,0,0,0,1)$ & $(0,1) \oplus(0,2)$ & $(1,2,1,0,0,1)$ & $(0,0) \oplus 2(0,1) \oplus(0,2)$ \\
\hline$(1,2,1,1,0,1)$ & $(0,0) \oplus 2(0,1) \oplus(0,2)$ & $(1,2,2,0,0,1)$ & $(0,0) \oplus 2(0,1) \oplus(0,2)$ \\
\hline$(1,3,0,0,0,1)$ & $(0,2) \oplus(0,3)$ & $(1,3,1,0,0,1)$ & $(0,1) \oplus 2(0,2) \oplus(0,3)$ \\
\hline$(1,4,0,0,0,1)$ & $(0,3) \oplus(0,4)$ & $(2,1,0,0,0,1)$ & $(0,1)$ \\
\hline$(2,2,0,0,0,1)$ & $(0,0) \oplus(0,1) \oplus(0,2)$ & $(2,2,1,0,0,1)$ & $(0,0) \oplus 2(0,1) \oplus(0,2)$ \\
\hline$(2,3,0,0,0,1)$ & $(0,1) \oplus(0,2) \oplus(0,3)$ & $(3,1,0,0,0,1)$ & $(0,2)$ \\
\hline$(3,2,0,0,0,1)$ & $(0,1) \oplus(0,2)$ & $(4,1,0,0,0,1)$ & $(0,3)$ \\
\hline$(0,0,2,0,0,2)$ & $(0,5 / 2)$ & $\begin{array}{l}(0,0,2,1,0,2) \\
\end{array}$ & $(0,3 / 2) \oplus(0,5 / 2)$ \\
\hline$(0,0,2,1,1,2)$ & $(0,3 / 2) \oplus(0,5 / 2)$ & \begin{tabular}{|l|}
$(0,0,2,2,0,2)$ \\
\end{tabular} & $(0,1 / 2) \oplus(0,3 / 2) \oplus(0,5 / 2)$ \\
\hline$(0,0,3,0,0,2)$ & $(0,5 / 2) \oplus(0,7 / 2) \oplus(1 / 2,4)$ & $(0,0,3,1,0,2)$ & $\begin{array}{l}(0,3 / 2) \oplus 3(0,5 / 2) \oplus 2(0,7 / 2) \oplus(1 / 2,3) \oplus \\
(1 / 2,4)\end{array}$ \\
\hline$(0,0,4,0,0,2)$ & $\begin{array}{l}(0,5 / 2) \oplus(0,7 / 2) \oplus 2(0,9 / 2) \oplus(1 / 2,4) \oplus \\
(1 / 2,5) \oplus(1,11 / 2)\end{array}$ & $(0,1,2,0,0,2)$ & $(0,3 / 2) \oplus(0,5 / 2)$ \\
\hline$(0,1,2,1,0,2)$ & $(0,1 / 2) \oplus 2(0,3 / 2) \oplus(0,5 / 2)$ & $(0,1,3,0,0,2)$ & $\begin{array}{l}(0,3 / 2) \oplus 3(0,5 / 2) \oplus 2(0,7 / 2) \oplus(1 / 2,3) \oplus \\
(1 / 2,4)\end{array}$ \\
\hline$(0,2,0,0,0,2)$ & $(0,5 / 2)$ & $(0,2,1,0,0,2)$ & $(0,3 / 2) \oplus(0,5 / 2)$ \\
\hline$(0,2,1,1,0,2)$ & $(0,3 / 2) \oplus(0,5 / 2)$ & $(0,2,2,0,0,2)$ & $2(0,1 / 2) \oplus 2(0,3 / 2) \oplus 2(0,5 / 2) \oplus(0,7 / 2)$ \\
\hline$(0,3,0,0,0,2)$ & $(0,5 / 2) \oplus(0,7 / 2) \oplus(1 / 2,4)$ & $(0,3,1,0,0,2)$ & $\begin{array}{l}(0,3 / 2) \oplus 3(0,5 / 2) \oplus 2(0,7 / 2) \oplus(1 / 2,3) \oplus \\
(1 / 2,4)\end{array}$ \\
\hline$(0,4,0,0,0,2)$ & $\begin{array}{l}(0,5 / 2) \oplus(0,7 / 2) \oplus 2(0,9 / 2) \oplus(1 / 2,4) \oplus \\
(1 / 2,5) \oplus(1,11 / 2)\end{array}$ & $(1,1,2,0,0,2)$ & $(0,3 / 2) \oplus(0,5 / 2)$ \\
\hline
\end{tabular}

continued on next page 


\begin{tabular}{|c|l|c|c|}
\hline$\beta$ & \multicolumn{1}{|c|}{$\oplus N_{j_{l}, j_{r}}^{\beta}\left(j_{l}, j_{r}\right)$} & \multicolumn{1}{|c|}{$\beta$} & \multicolumn{1}{|c|}{$\oplus N_{j_{l}, j_{r}}^{\beta}\left(j_{l}, j_{r}\right)$} \\
\hline$(1,2,0,0,0,2)$ & $(0,3 / 2) \oplus(0,5 / 2)$ & $(1,2,1,0,0,2)$ & $(0,1 / 2) \oplus 2(0,3 / 2) \oplus(0,5 / 2)$ \\
\hline$(1,3,0,0,0,2)$ & $\begin{array}{l}(0,3 / 2) \oplus 3(0,5 / 2) \oplus 2(0,7 / 2) \oplus(1 / 2,3) \oplus \\
(1 / 2,4)\end{array}$ & $(2,2,0,0,0,2)$ & $(0,1 / 2) \oplus(0,3 / 2) \oplus(0,5 / 2)$ \\
\hline$(0,0,3,0,0,3)$ & $(0,3) \oplus(1 / 2,9 / 2)$ & $(0,3,0,0,0,3)$ & $(0,3) \oplus(1 / 2,9 / 2)$ \\
\hline
\end{tabular}

Table 24. Refined BPS invariants of $6 \mathrm{~d} F_{4}$ minimal SCFT.

\begin{tabular}{|c|c|c|c|}
\hline$\beta$ & $\oplus N_{j_{l}, j_{r}}^{\beta}\left(j_{l}, j_{r}\right)$ & $\beta$ & $\oplus N_{j_{l}, j_{r}}^{\beta}\left(j_{l}, j_{r}\right)$ \\
\hline$(0,0,0,0,0,0,0,1)$ & $(0,1 / 2)$ & $(0,1,0,0,0,0,0,1)$ & $(0,1 / 2)$ \\
\hline$(0,2,0,0,0,0,0,1)$ & $(0,3 / 2)$ & $(0,3,0,0,0,0,0,1)$ & $(0,5 / 2)$ \\
\hline$(0,4,0,0,0,0,0,1)$ & $(0,7 / 2)$ & $(0,5,0,0,0,0,0,1)$ & $(0,9 / 2)$ \\
\hline$(0,0,0,0,0,1,0,1)$ & $(0,1 / 2)$ & $(0,0,0,0,0,1,1,1)$ & $(0,1 / 2)$ \\
\hline$(0,0,0,0,0,2,0,1)$ & $(0,3 / 2)$ & $(0,0,0,0,0,2,1,1)$ & $(0,1 / 2) \oplus(0,3 / 2)$ \\
\hline$(0,0,0,0,0,2,2,1)$ & $(0,1 / 2) \oplus(0,3 / 2)$ & $(0,0,0,0,0,2,3,1)$ & $(0,3 / 2)$ \\
\hline$(0,0,0,0,0,3,0,1)$ & $(0,5 / 2)$ & $(0,0,0,0,0,3,1,1)$ & $(0,3 / 2) \oplus(0,5 / 2)$ \\
\hline$(0,0,0,0,0,3,2,1)$ & $(0,1 / 2) \oplus(0,3 / 2) \oplus(0,5 / 2)$ & $(0,0,0,0,0,4,0,1)$ & $(0,7 / 2)$ \\
\hline$(0,0,0,0,0,4,1,1)$ & $(0,5 / 2) \oplus(0,7 / 2)$ & $(0,0,0,0,0,5,0,1)$ & $(0,9 / 2)$ \\
\hline$(0,0,0,0,1,0,0,1)$ & $(0,3 / 2)$ & $(0,1,0,0,1,0,0,1)$ & $(0,1 / 2) \oplus(0,3 / 2)$ \\
\hline$(0,2,0,0,1,0,0,1)$ & $(0,1 / 2) \oplus(0,3 / 2)$ & $(0,3,0,0,1,0,0,1)$ & $(0,3 / 2) \oplus(0,5 / 2)$ \\
\hline$(0,4,0,0,1,0,0,1)$ & $(0,5 / 2) \oplus(0,7 / 2)$ & $(0,0,0,0,1,1,0,1)$ & $(0,1 / 2) \oplus(0,3 / 2)$ \\
\hline$(0,1,0,0,1,1,0,1)$ & $2(0,1 / 2) \oplus(0,3 / 2)$ & $(0,2,0,0,1,1,0,1)$ & $(0,1 / 2) \oplus(0,3 / 2)$ \\
\hline$(0,3,0,0,1,1,0,1)$ & $(0,3 / 2) \oplus(0,5 / 2)$ & $(0,0,0,0,1,1,1,1)$ & $(0,1 / 2) \oplus(0,3 / 2)$ \\
\hline$(0,1,0,0,1,1,1,1)$ & $2(0,1 / 2) \oplus(0,3 / 2)$ & $(0,2,0,0,1,1,1,1)$ & $(0,1 / 2) \oplus(0,3 / 2)$ \\
\hline$(0,0,0,0,1,2,0,1)$ & $(0,1 / 2) \oplus(0,3 / 2)$ & $(0,1,0,0,1,2,0,1)$ & $(0,1 / 2) \oplus(0,3 / 2)$ \\
\hline$(0,0,0,0,1,2,1,1)$ & $2(0,1 / 2) \oplus(0,3 / 2)$ & $(0,1,0,0,1,2,1,1)$ & $2(0,1 / 2) \oplus(0,3 / 2)$ \\
\hline$(0,0,0,0,1,2,2,1)$ & $(0,1 / 2) \oplus(0,3 / 2)$ & $(0,0,0,0,1,3,0,1)$ & $(0,3 / 2) \oplus(0,5 / 2)$ \\
\hline$(0,1,0,0,1,3,0,1)$ & $(0,3 / 2) \oplus(0,5 / 2)$ & $(0,0,0,0,1,3,1,1)$ & $(0,1 / 2) \oplus 2(0,3 / 2) \oplus(0,5 / 2)$ \\
\hline$(0,0,0,0,1,4,0,1)$ & $(0,5 / 2) \oplus(0,7 / 2)$ & $(0,0,0,0,2,0,0,1)$ & $(0,5 / 2)$ \\
\hline$(0,1,0,0,2,0,0,1)$ & $(0,3 / 2) \oplus(0,5 / 2)$ & $(0,2,0,0,2,0,0,1)$ & $(0,1 / 2) \oplus(0,3 / 2) \oplus(0,5 / 2)$ \\
\hline$(0,3,0,0,2,0,0,1)$ & $(0,1 / 2) \oplus(0,3 / 2) \oplus(0,5 / 2)$ & $(0,0,0,0,2,1,0,1)$ & $(0,3 / 2) \oplus(0,5 / 2)$ \\
\hline$(0,1,0,0,2,1,0,1)$ & $(0,1 / 2) \oplus 2(0,3 / 2) \oplus(0,5 / 2)$ & $(0,2,0,0,2,1,0,1)$ & $2(0,1 / 2) \oplus 2(0,3 / 2) \oplus(0,5 / 2)$ \\
\hline$(0,0,0,0,2,1,1,1)$ & $(0,3 / 2) \oplus(0,5 / 2)$ & $(0,1,0,0,2,1,1,1)$ & $(0,1 / 2) \oplus 2(0,3 / 2) \oplus(0,5 / 2)$ \\
\hline$(0,0,0,0,2,2,0,1)$ & $(0,1 / 2) \oplus(0,3 / 2) \oplus(0,5 / 2)$ & $(0,1,0,0,2,2,0,1)$ & $2(0,1 / 2) \oplus 2(0,3 / 2) \oplus(0,5 / 2)$ \\
\hline$(0,0,0,0,2,2,1,1)$ & $(0,1 / 2) \oplus 2(0,3 / 2) \oplus(0,5 / 2)$ & $(0,0,0,0,2,3,0,1)$ & $(0,1 / 2) \oplus(0,3 / 2) \oplus(0,5 / 2)$ \\
\hline$(0,0,0,0,3,0,0,1)$ & $(0,7 / 2)$ & $(0,1,0,0,3,0,0,1)$ & $(0,5 / 2) \oplus(0,7 / 2)$ \\
\hline$(0,2,0,0,3,0,0,1)$ & $(0,3 / 2) \oplus(0,5 / 2) \oplus(0,7 / 2)$ & $(0,0,0,0,3,1,0,1)$ & $(0,5 / 2) \oplus(0,7 / 2)$ \\
\hline$(0,1,0,0,3,1,0,1)$ & $(0,3 / 2) \oplus 2(0,5 / 2) \oplus(0,7 / 2)$ & $(0,0,0,0,3,1,1,1)$ & $(0,5 / 2) \oplus(0,7 / 2)$ \\
\hline$(0,0,0,0,3,2,0,1)$ & $(0,3 / 2) \oplus(0,5 / 2) \oplus(0,7 / 2)$ & $(0,0,0,0,4,0,0,1)$ & $(0,9 / 2)$ \\
\hline$(0,1,0,0,4,0,0,1)$ & $(0,7 / 2) \oplus(0,9 / 2)$ & $(0,0,0,0,4,1,0,1)$ & $(0,7 / 2) \oplus(0,9 / 2)$ \\
\hline$(0,0,0,0,5,0,0,1)$ & $(0,11 / 2)$ & $(0,0,0,1,0,0,0,1)$ & $(0,1 / 2)$ \\
\hline$(0,0,0,1,1,0,0,1)$ & $(0,1 / 2) \oplus(0,3 / 2)$ & $(0,1,0,1,1,0,0,1)$ & $2(0,1 / 2) \oplus(0,3 / 2)$ \\
\hline$(0,2,0,1,1,0,0,1)$ & $(0,1 / 2) \oplus(0,3 / 2)$ & $(0,3,0,1,1,0,0,1)$ & $(0,3 / 2) \oplus(0,5 / 2)$ \\
\hline$(0,0,0,1,1,1,0,1)$ & $2(0,1 / 2) \oplus(0,3 / 2)$ & $(0,1,0,1,1,1,0,1)$ & $3(0,1 / 2) \oplus(0,3 / 2)$ \\
\hline$(0,2,0,1,1,1,0,1)$ & $(0,1 / 2) \oplus(0,3 / 2)$ & $(0,0,0,1,1,1,1,1)$ & $2(0,1 / 2) \oplus(0,3 / 2)$ \\
\hline$(0,1,0,1,1,1,1,1)$ & $3(0,1 / 2) \oplus(0,3 / 2)$ & $(0,0,0,1,1,2,0,1)$ & $(0,1 / 2) \oplus(0,3 / 2)$ \\
\hline$(0,1,0,1,1,2,0,1)$ & $(0,1 / 2) \oplus(0,3 / 2)$ & $(0,0,0,1,1,2,1,1)$ & $2(0,1 / 2) \oplus(0,3 / 2)$ \\
\hline$(0,0,0,1,1,3,0,1)$ & $(0,3 / 2) \oplus(0,5 / 2)$ & $(0,0,0,1,2,0,0,1)$ & $(0,3 / 2) \oplus(0,5 / 2)$ \\
\hline$(0,1,0,1,2,0,0,1)$ & $(0,1 / 2) \oplus 2(0,3 / 2) \oplus(0,5 / 2)$ & $(0,2,0,1,2,0,0,1)$ & $2(0,1 / 2) \oplus 2(0,3 / 2) \oplus(0,5 / 2)$ \\
\hline
\end{tabular}




\begin{tabular}{|c|c|c|c|}
\hline$\beta$ & $\oplus N_{j_{l}, j_{r}}^{\beta}\left(j_{l}, j_{r}\right)$ & $\beta$ & $\oplus N_{j_{l}, j_{r}}^{\beta}\left(j_{l}, j_{r}\right)$ \\
\hline$(0,0,0,1,2,1,0,1)$ & $(0,1 / 2) \oplus 2(0,3 / 2) \oplus(0,5 / 2)$ & \begin{tabular}{|l|}
$(0,1,0,1,2,1,0,1)$ \\
\end{tabular} & $4(0,1 / 2) \oplus 4(0,3 / 2) \oplus(0,5 / 2)$ \\
\hline$(0,0,0,1,2,1,1,1)$ & $(0,1 / 2) \oplus 2(0,3 / 2) \oplus(0,5 / 2)$ & $(0,0,0,1,2,2,0,1)$ & $2(0,1 / 2) \oplus 2(0,3 / 2) \oplus(0,5 / 2)$ \\
\hline$(0,0,0,1,3,0,0,1)$ & $(0,5 / 2) \oplus(0,7 / 2)$ & $(0,1,0,1,3,0,0,1)$ & $(0,3 / 2) \oplus 2(0,5 / 2) \oplus(0,7 / 2)$ \\
\hline$(0,0,0,1,3,1,0,1)$ & $(0,3 / 2) \oplus 2(0,5 / 2) \oplus(0,7 / 2)$ & $(0,0,0,1,4,0,0,1)$ & $(0,7 / 2) \oplus(0,9 / 2)$ \\
\hline$(0,0,0,2,0,0,0,1)$ & $(0,3 / 2)$ & $(0,0,0,2,1,0,0,1)$ & $(0,1 / 2) \oplus(0,3 / 2)$ \\
\hline$(0,1,0,2,1,0,0,1)$ & $(0,1 / 2) \oplus(0,3 / 2)$ & $(0,0,0,2,1,1,0,1)$ & $(0,1 / 2) \oplus(0,3 / 2)$ \\
\hline$(0,1,0,2,1,1,0,1)$ & $(0,1 / 2) \oplus(0,3 / 2)$ & $(0,0,0,2,1,1,1,1)$ & $(0,1 / 2) \oplus(0,3 / 2)$ \\
\hline$(0,0,0,2,2,0,0,1)$ & $(0,1 / 2) \oplus(0,3 / 2) \oplus(0,5 / 2)$ & $(0,1,0,2,2,0,0,1)$ & $2(0,1 / 2) \oplus 2(0,3 / 2) \oplus(0,5 / 2)$ \\
\hline$(0,0,0,2,2,1,0,1)$ & $2(0,1 / 2) \oplus 2(0,3 / 2) \oplus(0,5 / 2)$ & $(0,0,0,2,3,0,0,1)$ & $(0,3 / 2) \oplus(0,5 / 2) \oplus(0,7 / 2)$ \\
\hline$(0,0,0,3,0,0,0,1)$ & $(0,5 / 2)$ & $(0,0,0,3,1,0,0,1)$ & $(0,3 / 2) \oplus(0,5 / 2)$ \\
\hline$(0,1,0,3,1,0,0,1)$ & $(0,3 / 2) \oplus(0,5 / 2)$ & $(0,0,0,3,1,1,0,1)$ & $(0,3 / 2) \oplus(0,5 / 2)$ \\
\hline$(0,0,0,3,2,0,0,1)$ & $(0,1 / 2) \oplus(0,3 / 2) \oplus(0,5 / 2)$ & $(0,0,0,4,0,0,0,1)$ & $(0,7 / 2)$ \\
\hline$(0,0,0,4,1,0,0,1)$ & $(0,5 / 2) \oplus(0,7 / 2)$ & $(0,0,0,5,0,0,0,1)$ & $(0,9 / 2)$ \\
\hline$(0,0,1,1,0,0,0,1)$ & $(0,1 / 2)$ & $(0,0,1,1,1,0,0,1)$ & $(0,1 / 2) \oplus(0,3 / 2)$ \\
\hline$(0,1,1,1,1,0,0,1)$ & $2(0,1 / 2) \oplus(0,3 / 2)$ & $(0,2,1,1,1,0,0,1)$ & $(0,1 / 2) \oplus(0,3 / 2)$ \\
\hline$(0,0,1,1,1,1,0,1)$ & $2(0,1 / 2) \oplus(0,3 / 2)$ & $(0,1,1,1,1,1,0,1)$ & $3(0,1 / 2) \oplus(0,3 / 2)$ \\
\hline$(0,0,1,1,1,1,1,1)$ & $2(0,1 / 2) \oplus(0,3 / 2)$ & $(0,0,1,1,1,2,0,1)$ & $(0,1 / 2) \oplus(0,3 / 2)$ \\
\hline$(0,0,1,1,2,0,0,1)$ & $(0,3 / 2) \oplus(0,5 / 2)$ & $(0,1,1,1,2,0,0,1)$ & $(0,1 / 2) \oplus 2(0,3 / 2) \oplus(0,5 / 2)$ \\
\hline$(0,0,1,1,2,1,0,1)$ & $(0,1 / 2) \oplus 2(0,3 / 2) \oplus(0,5 / 2)$ & $(0,0,1,1,3,0,0,1)$ & $(0,5 / 2) \oplus(0,7 / 2)$ \\
\hline$(0,0,1,2,0,0,0,1)$ & $(0,1 / 2) \oplus(0,3 / 2)$ & $(0,0,1,2,1,0,0,1)$ & $2(0,1 / 2) \oplus(0,3 / 2)$ \\
\hline$(0,1,1,2,1,0,0,1)$ & $2(0,1 / 2) \oplus(0,3 / 2)$ & $(0,0,1,2,1,1,0,1)$ & $2(0,1 / 2) \oplus(0,3 / 2)$ \\
\hline$(0,0,1,2,2,0,0,1)$ & $(0,1 / 2) \oplus 2(0,3 / 2) \oplus(0,5 / 2)$ & $(0,0,1,3,0,0,0,1)$ & $(0,3 / 2) \oplus(0,5 / 2)$ \\
\hline$(0,0,1,3,1,0,0,1)$ & $(0,1 / 2) \oplus 2(0,3 / 2) \oplus(0,5 / 2)$ & $(0,0,1,4,0,0,0,1)$ & $(0,5 / 2) \oplus(0,7 / 2)$ \\
\hline$(0,0,2,2,0,0,0,1)$ & $(0,1 / 2) \oplus(0,3 / 2)$ & $(0,0,2,2,1,0,0,1)$ & $(0,1 / 2) \oplus(0,3 / 2)$ \\
\hline$(0,0,2,3,0,0,0,1)$ & $(0,1 / 2) \oplus(0,3 / 2) \oplus(0,5 / 2)$ & $(0,0,3,2,0,0,0,1)$ & $(0,3 / 2)$ \\
\hline$(1,1,0,0,0,0,0,1)$ & $(0,1 / 2)$ & $(1,2,0,0,0,0,0,1)$ & $(0,1 / 2) \oplus(0,3 / 2)$ \\
\hline$(1,3,0,0,0,0,0,1)$ & $(0,3 / 2) \oplus(0,5 / 2)$ & $(1,4,0,0,0,0,0,1)$ & $(0,5 / 2) \oplus(0,7 / 2)$ \\
\hline$(1,1,0,0,1,0,0,1)$ & $(0,1 / 2) \oplus(0,3 / 2)$ & $(1,2,0,0,1,0,0,1)$ & $2(0,1 / 2) \oplus(0,3 / 2)$ \\
\hline$(1,3,0,0,1,0,0,1)$ & $(0,1 / 2) \oplus 2(0,3 / 2) \oplus(0,5 / 2)$ & $(1,1,0,0,1,1,0,1)$ & $2(0,1 / 2) \oplus(0,3 / 2)$ \\
\hline$(1,2,0,0,1,1,0,1)$ & $2(0,1 / 2) \oplus(0,3 / 2)$ & $(1,1,0,0,1,1,1,1)$ & $2(0,1 / 2) \oplus(0,3 / 2)$ \\
\hline$(1,1,0,0,1,2,0,1)$ & $(0,1 / 2) \oplus(0,3 / 2)$ & $(1,1,0,0,2,0,0,1)$ & $(0,3 / 2) \oplus(0,5 / 2)$ \\
\hline$(1,2,0,0,2,0,0,1)$ & $(0,1 / 2) \oplus 2(0,3 / 2) \oplus(0,5 / 2)$ & $(1,1,0,0,2,1,0,1)$ & $(0,1 / 2) \oplus 2(0,3 / 2) \oplus(0,5 / 2)$ \\
\hline$(1,1,0,0,3,0,0,1)$ & $(0,5 / 2) \oplus(0,7 / 2)$ & $(1,1,0,1,1,0,0,1)$ & $2(0,1 / 2) \oplus(0,3 / 2)$ \\
\hline$(1,2,0,1,1,0,0,1)$ & $2(0,1 / 2) \oplus(0,3 / 2)$ & $(1,1,0,1,1,1,0,1)$ & $3(0,1 / 2) \oplus(0,3 / 2)$ \\
\hline$(1,1,0,1,2,0,0,1)$ & $(0,1 / 2) \oplus 2(0,3 / 2) \oplus(0,5 / 2)$ & $(1,1,0,2,1,0,0,1)$ & $(0,1 / 2) \oplus(0,3 / 2)$ \\
\hline$(1,1,1,1,1,0,0,1)$ & $2(0,1 / 2) \oplus(0,3 / 2)$ & $(2,2,0,0,0,0,0,1)$ & $(0,1 / 2) \oplus(0,3 / 2)$ \\
\hline$(2,3,0,0,0,0,0,1)$ & $(0,1 / 2) \oplus(0,3 / 2) \oplus(0,5 / 2)$ & $(2,2,0,0,1,0,0,1)$ & $(0,1 / 2) \oplus(0,3 / 2)$ \\
\hline$(3,2,0,0,0,0,0,1)$ & $(0,3 / 2)$ & $(0,3,0,0,0,0,0,2)$ & $(0,5 / 2)$ \\
\hline$(0,4,0,0,0,0,0,2)$ & $(0,5 / 2) \oplus(0,7 / 2) \oplus(1 / 2,4)$ & $(0,0,0,0,0,3,0,2)$ & $(0,5 / 2)$ \\
\hline$(0,0,0,0,0,3,1,2)$ & $(0,3 / 2) \oplus(0,5 / 2)$ & $(0,0,0,0,0,4,0,2)$ & $(0,5 / 2) \oplus(0,7 / 2) \oplus(1 / 2,4)$ \\
\hline$(0,0,0,0,1,0,0,2)$ & $(0,5 / 2)$ & $(0,1,0,0,1,0,0,2)$ & $(0,3 / 2) \oplus(0,5 / 2)$ \\
\hline$(0,2,0,0,1,0,0,2)$ & $(0,1 / 2) \oplus(0,3 / 2) \oplus(0,5 / 2)$ & $(0,3,0,0,1,0,0,2)$ & $\begin{array}{l}(0,1 / 2) \oplus 2(0,3 / 2) \oplus 2(0,5 / 2) \oplus \\
(0,7 / 2)\end{array}$ \\
\hline$(0,0,0,0,1,1,0,2)$ & $(0,3 / 2) \oplus(0,5 / 2)$ & $(0,1,0,0,1,1,0,2)$ & $(0,1 / 2) \oplus 2(0,3 / 2) \oplus(0,5 / 2)$ \\
\hline$(0,2,0,0,1,1,0,2)$ & $2(0,1 / 2) \oplus 2(0,3 / 2) \oplus(0,5 / 2)$ & $(0,0,0,0,1,1,1,2)$ & $(0,3 / 2) \oplus(0,5 / 2)$ \\
\hline$(0,1,0,0,1,1,1,2)$ & $(0,1 / 2) \oplus 2(0,3 / 2) \oplus(0,5 / 2)$ & $(0,0,0,0,1,2,0,2)$ & $(0,1 / 2) \oplus(0,3 / 2) \oplus(0,5 / 2)$ \\
\hline$(0,1,0,0,1,2,0,2)$ & $2(0,1 / 2) \oplus 2(0,3 / 2) \oplus(0,5 / 2)$ & $(0,0,0,0,1,2,1,2)$ & $(0,1 / 2) \oplus 2(0,3 / 2) \oplus(0,5 / 2)$ \\
\hline$(0,0,0,0,1,3,0,2)$ & $\begin{array}{l}(0,1 / 2) \oplus 2(0,3 / 2) \oplus 2(0,5 / 2) \oplus \\
(0,7 / 2)\end{array}$ & \begin{tabular}{|l|l|}
$(0,0,0,0,2,0,0,2)$ \\
\end{tabular} & $(0,5 / 2) \oplus(0,7 / 2) \oplus(1 / 2,4)$ \\
\hline
\end{tabular}




\begin{tabular}{|c|c|c|c|}
\hline$\beta$ & $\oplus N_{j_{l}, j_{r}}^{\beta}\left(j_{l}, j_{r}\right)$ & $\beta$ & $\oplus N_{j_{l}, j_{r}}^{\beta}\left(j_{l}, j_{r}\right)$ \\
\hline$(0,1,0,0,2,0,0,2)$ & $\begin{array}{l}(0,3 / 2) \oplus 3(0,5 / 2) \oplus 2(0,7 / 2) \oplus \\
(1 / 2,3) \oplus(1 / 2,4)\end{array}$ & $(0,2,0,0,2,0,0,2)$ & $\begin{array}{l}(0,1 / 2) \oplus 3(0,3 / 2) \oplus 4(0,5 / 2) \oplus \\
2(0,7 / 2) \oplus(1 / 2,2) \oplus(1 / 2,3) \oplus(1 / 2,4)\end{array}$ \\
\hline$(0,0,0,0,2,1,0,2)$ & $\begin{array}{l}(0,3 / 2) \oplus 3(0,5 / 2) \oplus 2(0,7 / 2) \oplus \\
(1 / 2,3) \oplus(1 / 2,4)\end{array}$ & $(0,1,0,0,2,1,0,2)$ & $\begin{array}{l}(0,1 / 2) \oplus 5(0,3 / 2) \oplus 7(0,5 / 2) \oplus \\
3(0,7 / 2) \oplus(1 / 2,2) \oplus 2(1 / 2,3) \oplus \\
(1 / 2,4)\end{array}$ \\
\hline$(0,0,0,0,2,1,1,2)$ & $\begin{array}{l}(0,3 / 2) \oplus 3(0,5 / 2) \oplus 2(0,7 / 2) \oplus \\
(1 / 2,3) \oplus(1 / 2,4)\end{array}$ & $(0,0,0,0,2,2,0,2)$ & $\begin{array}{l}(0,1 / 2) \oplus 3(0,3 / 2) \oplus 4(0,5 / 2) \oplus \\
2(0,7 / 2) \oplus(1 / 2,2) \oplus(1 / 2,3) \oplus(1 / 2,4)\end{array}$ \\
\hline$(0,0,0,0,3,0,0,2)$ & $\begin{array}{l}(0,5 / 2) \oplus(0,7 / 2) \oplus 2(0,9 / 2) \oplus \\
(1 / 2,4) \oplus(1 / 2,5) \oplus(1,11 / 2)\end{array}$ & $(0,1,0,0,3,0,0,2)$ & $\begin{array}{l}(0,3 / 2) \oplus 3(0,5 / 2) \oplus 5(0,7 / 2) \oplus \\
3(0,9 / 2) \oplus(1 / 2,3) \oplus 3(1 / 2,4) \oplus \\
2(1 / 2,5) \oplus(1,9 / 2) \oplus(1,11 / 2)\end{array}$ \\
\hline$(0,0,0,0,3,1,0,2)$ & $\begin{array}{l}(0,3 / 2) \oplus 3(0,5 / 2) \oplus 5(0,7 / 2) \oplus \\
3(0,9 / 2) \oplus(1 / 2,3) \oplus 3(1 / 2,4) \oplus \\
2(1 / 2,5) \oplus(1,9 / 2) \oplus(1,11 / 2)\end{array}$ & $(0,0,0,0,4,0,0,2)$ & $\begin{array}{l}(0,5 / 2) \oplus(0,7 / 2) \oplus 2(0,9 / 2) \oplus \\
2(0,11 / 2) \oplus(1 / 2,4) \oplus(1 / 2,5) \oplus \\
2(1 / 2,6) \oplus(1,11 / 2) \oplus(1,13 / 2) \oplus \\
(3 / 2,7)\end{array}$ \\
\hline$(0,0,0,1,1,0,0,2)$ & $(0,3 / 2) \oplus(0,5 / 2)$ & $(0,1,0,1,1,0,0,2)$ & $(0,1 / 2) \oplus 2(0,3 / 2) \oplus(0,5 / 2)$ \\
\hline$(0,2,0,1,1,0,0,2)$ & $2(0,1 / 2) \oplus 2(0,3 / 2) \oplus(0,5 / 2)$ & $(0,0,0,1,1,1,0,2)$ & $(0,1 / 2) \oplus 2(0,3 / 2) \oplus(0,5 / 2)$ \\
\hline$(0,1,0,1,1,1,0,2)$ & $3(0,1 / 2) \oplus 3(0,3 / 2) \oplus(0,5 / 2)$ & $(0,0,0,1,1,1,1,2)$ & $(0,1 / 2) \oplus 2(0,3 / 2) \oplus(0,5 / 2)$ \\
\hline$(0,0,0,1,1,2,0,2)$ & $2(0,1 / 2) \oplus 2(0,3 / 2) \oplus(0,5 / 2)$ & $(0,0,0,1,2,0,0,2)$ & $\begin{array}{l}(0,3 / 2) \oplus 3(0,5 / 2) \oplus 2(0,7 / 2) \oplus \\
(1 / 2,3) \oplus(1 / 2,4)\end{array}$ \\
\hline$(0,1,0,1,2,0,0,2)$ & $\begin{array}{l}(0,1 / 2) \oplus 5(0,3 / 2) \oplus 7(0,5 / 2) \oplus \\
3(0,7 / 2) \oplus(1 / 2,2) \oplus 2(1 / 2,3) \oplus \\
(1 / 2,4)\end{array}$ & $(0,0,0,1,2,1,0,2)$ & $\begin{array}{l}(0,1 / 2) \oplus 5(0,3 / 2) \oplus 7(0,5 / 2) \oplus \\
3(0,7 / 2) \oplus(1 / 2,2) \oplus 2(1 / 2,3) \oplus \\
(1 / 2,4)\end{array}$ \\
\hline$(0,0,0,1,3,0,0,2)$ & $\begin{array}{l}(0,3 / 2) \oplus 3(0,5 / 2) \oplus 5(0,7 / 2) \oplus \\
3(0,9 / 2) \oplus(1 / 2,3) \oplus 3(1 / 2,4) \oplus \\
2(1 / 2,5) \oplus(1,9 / 2) \oplus(1,11 / 2)\end{array}$ & $(0,0,0,2,1,0,0,2)$ & $(0,1 / 2) \oplus(0,3 / 2) \oplus(0,5 / 2)$ \\
\hline$(0,1,0,2,1,0,0,2)$ & $2(0,1 / 2) \oplus 2(0,3 / 2) \oplus(0,5 / 2)$ & $(0,0,0,2,1,1,0,2)$ & $2(0,1 / 2) \oplus 2(0,3 / 2) \oplus(0,5 / 2)$ \\
\hline$(0,0,0,2,2,0,0,2)$ & $\begin{array}{l}(0,1 / 2) \oplus 3(0,3 / 2) \oplus 4(0,5 / 2) \oplus \\
2(0,7 / 2) \oplus(1 / 2,2) \oplus(1 / 2,3) \oplus(1 / 2,4)\end{array}$ & $(0,0,0,3,0,0,0,2)$ & $(0,5 / 2)$ \\
\hline$(0,0,0,3,1,0,0,2)$ & $\begin{array}{l}(0,1 / 2) \oplus 2(0,3 / 2) \oplus 2(0,5 / 2) \oplus \\
(0,7 / 2)\end{array}$ & $(0,0,0,4,0,0,0,2)$ & $(0,5 / 2) \oplus(0,7 / 2) \oplus(1 / 2,4)$ \\
\hline$(0,0,1,1,1,0,0,2)$ & $(0,3 / 2) \oplus(0,5 / 2)$ & $(0,1,1,1,1,0,0,2)$ & $(0,1 / 2) \oplus 2(0,3 / 2) \oplus(0,5 / 2)$ \\
\hline$(0,0,1,1,1,1,0,2)$ & $(0,1 / 2) \oplus 2(0,3 / 2) \oplus(0,5 / 2)$ & $(0,0,1,1,2,0,0,2)$ & $\begin{array}{l}(0,3 / 2) \oplus 3(0,5 / 2) \oplus 2(0,7 / 2) \oplus \\
(1 / 2,3) \oplus(1 / 2,4)\end{array}$ \\
\hline$(0,0,1,2,1,0,0,2)$ & $(0,1 / 2) \oplus 2(0,3 / 2) \oplus(0,5 / 2)$ & $(0,0,1,3,0,0,0,2)$ & $(0,3 / 2) \oplus(0,5 / 2)$ \\
\hline$(1,3,0,0,0,0,0,2)$ & $(0,3 / 2) \oplus(0,5 / 2)$ & $(1,1,0,0,1,0,0,2)$ & $(0,3 / 2) \oplus(0,5 / 2)$ \\
\hline$(1,2,0,0,1,0,0,2)$ & $(0,1 / 2) \oplus 2(0,3 / 2) \oplus(0,5 / 2)$ & $(1,1,0,0,1,1,0,2)$ & $(0,1 / 2) \oplus 2(0,3 / 2) \oplus(0,5 / 2)$ \\
\hline$(1,1,0,0,2,0,0,2)$ & $\begin{array}{l}(0,3 / 2) \oplus 3(0,5 / 2) \oplus 2(0,7 / 2) \oplus \\
(1 / 2,3) \oplus(1 / 2,4)\end{array}$ & $(1,1,0,1,1,0,0,2)$ & $(0,1 / 2) \oplus 2(0,3 / 2) \oplus(0,5 / 2)$ \\
\hline$(0,0,0,0,1,0,0,3)$ & $(0,7 / 2)$ & $(0,1,0,0,1,0,0,3)$ & $(0,5 / 2) \oplus(0,7 / 2)$ \\
\hline$(0,2,0,0,1,0,0,3)$ & $(0,3 / 2) \oplus(0,5 / 2) \oplus(0,7 / 2)$ & $(0,0,0,0,1,1,0,3)$ & $(0,5 / 2) \oplus(0,7 / 2)$ \\
\hline$(0,1,0,0,1,1,0,3)$ & $(0,3 / 2) \oplus 2(0,5 / 2) \oplus(0,7 / 2)$ & $(0,0,0,0,1,1,1,3)$ & $(0,5 / 2) \oplus(0,7 / 2)$ \\
\hline$(0,0,0,0,1,2,0,3)$ & $(0,3 / 2) \oplus(0,5 / 2) \oplus(0,7 / 2)$ & $(0,0,0,0,2,0,0,3)$ & $\begin{array}{l}(0,5 / 2) \oplus(0,7 / 2) \oplus 2(0,9 / 2) \oplus \\
(1 / 2,4) \oplus(1 / 2,5) \oplus(1,11 / 2)\end{array}$ \\
\hline$(0,1,0,0,2,0,0,3)$ & $\begin{array}{l}(0,3 / 2) \oplus 3(0,5 / 2) \oplus 5(0,7 / 2) \oplus \\
3(0,9 / 2) \oplus(1 / 2,3) \oplus 3(1 / 2,4) \oplus \\
2(1 / 2,5) \oplus(1,9 / 2) \oplus(1,11 / 2)\end{array}$ & $(0,0,0,0,2,1,0,3)$ & $\begin{array}{l}(0,3 / 2) \oplus 3(0,5 / 2) \oplus 5(0,7 / 2) \oplus \\
3(0,9 / 2) \oplus(1 / 2,3) \oplus 3(1 / 2,4) \oplus \\
2(1 / 2,5) \oplus(1,9 / 2) \oplus(1,11 / 2)\end{array}$ \\
\hline
\end{tabular}




\begin{tabular}{|c|l|l|l|}
\hline$\beta$ & \multicolumn{1}{|c|}{$\oplus N_{j_{l}, j_{r}}^{\beta}\left(j_{l}, j_{r}\right)$} & \multicolumn{1}{|c|}{$\beta$} & \multicolumn{1}{c|}{$\oplus N_{j_{l}, j_{r}}^{\beta}\left(j_{l}, j_{r}\right)$} \\
\hline$(0,0,0,0,3,0,0,3)$ & $(0,3 / 2) \oplus(0,5 / 2) \oplus 3(0,7 / 2) \oplus$ & $(0,0,0,1,1,0,0,3)$ & $(0,5 / 2) \oplus(0,7 / 2)$ \\
& $3(0,9 / 2) \oplus 4(0,11 / 2) \oplus(1 / 2,3) \oplus$ & & \\
& $2(1 / 2,4) \oplus 3(1 / 2,5) \oplus 3(1 / 2,6) \oplus$ & & \\
& $(1 / 2,7) \oplus(1,9 / 2) \oplus 2(1,11 / 2) \oplus$ & & \\
& $3(1,13 / 2) \oplus(3 / 2,6) \oplus(3 / 2,7) \oplus$ & & \\
& $(2,15 / 2)$ & & \\
\hline$(0,1,0,1,1,0,0,3)$ & $(0,3 / 2) \oplus 2(0,5 / 2) \oplus(0,7 / 2)$ & $(0,0,0,1,1,1,0,3)$ & $(0,3 / 2) \oplus 2(0,5 / 2) \oplus(0,7 / 2)$ \\
\hline$(0,0,0,1,2,0,0,3)$ & $(0,3 / 2) \oplus 3(0,5 / 2) \oplus 5(0,7 / 2) \oplus$ & $(0,0,0,2,1,0,0,3)$ & $(0,3 / 2) \oplus(0,5 / 2) \oplus(0,7 / 2)$ \\
& $3(0,9 / 2) \oplus(1 / 2,3) \oplus 3(1 / 2,4) \oplus$ & & \\
& $2(1 / 2,5) \oplus(1,9 / 2) \oplus(1,11 / 2)$ & & $(1,2)$ \\
\hline$(0,0,1,1,1,0,0,3)$ & $(0,5 / 2) \oplus(0,7 / 2)$ & $(0,1,0,0,1,0,0,4)$ & $(0,7 / 2) \oplus(0,9 / 2)$ \\
\hline$(0,0,0,0,1,0,0,4)$ & $(0,9 / 2)$ & $(0,0,0,0,2,0,0,4)$ & $(0,5 / 2) \oplus(0,7 / 2) \oplus 2(0,9 / 2) \oplus$ \\
\hline$(0,0,0,0,1,1,0,4)$ & $(0,7 / 2) \oplus(0,9 / 2)$ & & $2(0,11 / 2) \oplus(1 / 2,4) \oplus(1 / 2,5) \oplus$ \\
& & & $(1 / 2,6) \oplus(1,11 / 2) \oplus(1,13 / 2) \oplus$ \\
\hline$(0,0,0,1,1,0,0,4)$ & $(0,7 / 2) \oplus(0,9 / 2)$ & $(0,0,0,0,1,0,0,5)$ & $(0,11 / 2)$ \\
\hline
\end{tabular}

Table 25. Refined BPS invariants of $6 \mathrm{~d} E_{6}$ minimal SCFT.

\begin{tabular}{|c|c|c|c|}
\hline$\beta$ & $\oplus N_{j_{l}, j_{r}}^{\beta}\left(j_{l}, j_{r}\right)$ & $\beta$ & $\oplus N_{j_{l}, j_{r}}^{\beta}\left(j_{l}, j_{r}\right)$ \\
\hline$(0,0,0,0,0,0,0,0,1)$ & $(0,1 / 2)$ & $(0,0,0,0,0,0,0,1,1)$ & $(0,1 / 2)$ \\
\hline$(0,0,0,0,0,0,0,2,1)$ & $(0,3 / 2)$ & $(0,0,0,0,0,0,0,3,1)$ & $(0,5 / 2)$ \\
\hline$(0,0,0,0,0,0,0,4,1)$ & $(0,7 / 2)$ & $(0,0,0,0,0,0,0,5,1)$ & $(0,9 / 2)$ \\
\hline$(0,0,0,0,1,0,0,0,1)$ & $(0,1 / 2)$ & $(0,0,0,0,1,1,0,0,1)$ & $(0,1 / 2)$ \\
\hline$(0,0,0,0,1,1,1,0,1)$ & $(0,1 / 2)$ & $(0,0,0,0,2,0,0,0,1)$ & $(0,3 / 2)$ \\
\hline$(0,0,0,0,2,1,0,0,1)$ & $(0,1 / 2) \oplus(0,3 / 2)$ & $(0,0,0,0,2,1,1,0,1)$ & $(0,1 / 2) \oplus(0,3 / 2)$ \\
\hline$(0,0,0,0,2,2,0,0,1)$ & $(0,1 / 2) \oplus(0,3 / 2)$ & $(0,0,0,0,2,2,1,0,1)$ & $2(0,1 / 2) \oplus(0,3 / 2)$ \\
\hline$(0,0,0,0,2,3,0,0,1)$ & $(0,3 / 2)$ & $(0,0,0,0,3,0,0,0,1)$ & $(0,5 / 2)$ \\
\hline$(0,0,0,0,3,1,0,0,1)$ & $(0,3 / 2) \oplus(0,5 / 2)$ & $(0,0,0,0,3,1,1,0,1)$ & $(0,3 / 2) \oplus(0,5 / 2)$ \\
\hline$(0,0,0,0,3,2,0,0,1)$ & $(0,1 / 2) \oplus(0,3 / 2) \oplus(0,5 / 2)$ & $(0,0,0,0,4,0,0,0,1)$ & $(0,7 / 2)$ \\
\hline$(0,0,0,0,4,1,0,0,1)$ & $(0,5 / 2) \oplus(0,7 / 2)$ & $(0,0,0,0,5,0,0,0,1)$ & $(0,9 / 2)$ \\
\hline$(0,0,0,1,0,0,0,0,1)$ & $(0,3 / 2)$ & $(0,0,0,1,0,0,0,1,1)$ & $(0,1 / 2) \oplus(0,3 / 2)$ \\
\hline$(0,0,0,1,0,0,0,2,1)$ & $(0,1 / 2) \oplus(0,3 / 2)$ & $(0,0,0,1,0,0,0,3,1)$ & $(0,3 / 2) \oplus(0,5 / 2)$ \\
\hline$(0,0,0,1,0,0,0,4,1)$ & $(0,5 / 2) \oplus(0,7 / 2)$ & $(0,0,0,1,1,0,0,0,1)$ & $(0,1 / 2) \oplus(0,3 / 2)$ \\
\hline$(0,0,0,1,1,0,0,1,1)$ & $2(0,1 / 2) \oplus(0,3 / 2)$ & $(0,0,0,1,1,0,0,2,1)$ & $(0,1 / 2) \oplus(0,3 / 2)$ \\
\hline$(0,0,0,1,1,0,0,3,1)$ & $(0,3 / 2) \oplus(0,5 / 2)$ & $(0,0,0,1,1,1,0,0,1)$ & $(0,1 / 2) \oplus(0,3 / 2)$ \\
\hline$(0,0,0,1,1,1,0,1,1)$ & $2(0,1 / 2) \oplus(0,3 / 2)$ & $(0,0,0,1,1,1,0,2,1)$ & $(0,1 / 2) \oplus(0,3 / 2)$ \\
\hline$(0,0,0,1,1,1,1,0,1)$ & $(0,1 / 2) \oplus(0,3 / 2)$ & $(0,0,0,1,1,1,1,1,1)$ & $2(0,1 / 2) \oplus(0,3 / 2)$ \\
\hline$(0,0,0,1,2,0,0,0,1)$ & $(0,1 / 2) \oplus(0,3 / 2)$ & $(0,0,0,1,2,0,0,1,1)$ & $(0,1 / 2) \oplus(0,3 / 2)$ \\
\hline$(0,0,0,1,2,1,0,0,1)$ & $2(0,1 / 2) \oplus(0,3 / 2)$ & $(0,0,0,1,2,1,0,1,1)$ & $2(0,1 / 2) \oplus(0,3 / 2)$ \\
\hline$(0,0,0,1,2,1,1,0,1)$ & $2(0,1 / 2) \oplus(0,3 / 2)$ & $(0,0,0,1,2,2,0,0,1)$ & $(0,1 / 2) \oplus(0,3 / 2)$ \\
\hline$(0,0,0,1,3,0,0,0,1)$ & $(0,3 / 2) \oplus(0,5 / 2)$ & $(0,0,0,1,3,0,0,1,1)$ & $(0,3 / 2) \oplus(0,5 / 2)$ \\
\hline$(0,0,0,1,3,1,0,0,1)$ & $(0,1 / 2) \oplus 2(0,3 / 2) \oplus(0,5 / 2)$ & $(0,0,0,1,4,0,0,0,1)$ & $(0,5 / 2) \oplus(0,7 / 2)$ \\
\hline$(0,0,0,2,0,0,0,0,1)$ & $(0,5 / 2)$ & $(0,0,0,2,0,0,0,1,1)$ & $(0,3 / 2) \oplus(0,5 / 2)$ \\
\hline$(0,0,0,2,0,0,0,2,1)$ & $(0,1 / 2) \oplus(0,3 / 2) \oplus(0,5 / 2)$ & $(0,0,0,2,0,0,0,3,1)$ & $(0,1 / 2) \oplus(0,3 / 2) \oplus(0,5 / 2)$ \\
\hline$(0,0,0,2,1,0,0,0,1)$ & $(0,3 / 2) \oplus(0,5 / 2)$ & $(0,0,0,2,1,0,0,1,1)$ & $(0,1 / 2) \oplus 2(0,3 / 2) \oplus(0,5 / 2)$ \\
\hline$(0,0,0,2,1,0,0,2,1)$ & $2(0,1 / 2) \oplus 2(0,3 / 2) \oplus(0,5 / 2)$ & $(0,0,0,2,1,1,0,0,1)$ & $(0,3 / 2) \oplus(0,5 / 2)$ \\
\hline$(0,0,0,2,1,1,0,1,1)$ & $(0,1 / 2) \oplus 2(0,3 / 2) \oplus(0,5 / 2)$ & $(0,0,0,2,1,1,1,0,1)$ & $(0,3 / 2) \oplus(0,5 / 2)$ \\
\hline
\end{tabular}




\begin{tabular}{|c|c|c|c|}
\hline$\beta$ & $\oplus N_{j_{l}, j_{r}}^{\beta}\left(j_{l}, j_{r}\right)$ & $\beta$ & $\oplus N_{j_{l}, j_{r}}^{\beta}\left(j_{l}, j_{r}\right)$ \\
\hline$(0,0,0,2,2,0,0,0,1)$ & $(0,1 / 2) \oplus(0,3 / 2) \oplus(0,5 / 2)$ & $(0,0,0,2,2,0,0,1,1)$ & $2(0,1 / 2) \oplus 2(0,3 / 2) \oplus(0,5 / 2)$ \\
\hline$(0,0,0,2,2,1,0,0,1)$ & $(0,1 / 2) \oplus 2(0,3 / 2) \oplus(0,5 / 2)$ & $(0,0,0,2,3,0,0,0,1)$ & $(0,1 / 2) \oplus(0,3 / 2) \oplus(0,5 / 2)$ \\
\hline$(0,0,0,3,0,0,0,0,1)$ & $(0,7 / 2)$ & $(0,0,0,3,0,0,0,1,1)$ & $(0,5 / 2) \oplus(0,7 / 2)$ \\
\hline$(0,0,0,3,0,0,0,2,1)$ & $(0,3 / 2) \oplus(0,5 / 2) \oplus(0,7 / 2)$ & $(0,0,0,3,1,0,0,0,1)$ & $(0,5 / 2) \oplus(0,7 / 2)$ \\
\hline$(0,0,0,3,1,0,0,1,1)$ & $(0,3 / 2) \oplus 2(0,5 / 2) \oplus(0,7 / 2)$ & $(0,0,0,3,1,1,0,0,1)$ & $(0,5 / 2) \oplus(0,7 / 2)$ \\
\hline$(0,0,0,3,2,0,0,0,1)$ & $(0,3 / 2) \oplus(0,5 / 2) \oplus(0,7 / 2)$ & $(0,0,0,4,0,0,0,0,1)$ & $(0,9 / 2)$ \\
\hline$(0,0,0,4,0,0,0,1,1)$ & $(0,7 / 2) \oplus(0,9 / 2)$ & $(0,0,0,4,1,0,0,0,1)$ & $(0,7 / 2) \oplus(0,9 / 2)$ \\
\hline$(0,0,0,5,0,0,0,0,1)$ & $(0,11 / 2)$ & $(0,0,1,0,0,0,0,0,1)$ & $(0,1 / 2)$ \\
\hline$(0,0,1,1,0,0,0,0,1)$ & $(0,1 / 2) \oplus(0,3 / 2)$ & $(0,0,1,1,0,0,0,1,1)$ & $2(0,1 / 2) \oplus(0,3 / 2)$ \\
\hline$(0,0,1,1,0,0,0,2,1)$ & $(0,1 / 2) \oplus(0,3 / 2)$ & $(0,0,1,1,0,0,0,3,1)$ & $(0,3 / 2) \oplus(0,5 / 2)$ \\
\hline$(0,0,1,1,1,0,0,0,1)$ & $2(0,1 / 2) \oplus(0,3 / 2)$ & $(0,0,1,1,1,0,0,1,1)$ & $3(0,1 / 2) \oplus(0,3 / 2)$ \\
\hline$(0,0,1,1,1,0,0,2,1)$ & $(0,1 / 2) \oplus(0,3 / 2)$ & $(0,0,1,1,1,1,0,0,1)$ & $2(0,1 / 2) \oplus(0,3 / 2)$ \\
\hline$(0,0,1,1,1,1,0,1,1)$ & $3(0,1 / 2) \oplus(0,3 / 2)$ & $(0,0,1,1,1,1,1,0,1)$ & $2(0,1 / 2) \oplus(0,3 / 2)$ \\
\hline$(0,0,1,1,2,0,0,0,1)$ & $(0,1 / 2) \oplus(0,3 / 2)$ & $(0,0,1,1,2,0,0,1,1)$ & $(0,1 / 2) \oplus(0,3 / 2)$ \\
\hline$(0,0,1,1,2,1,0,0,1)$ & $2(0,1 / 2) \oplus(0,3 / 2)$ & $(0,0,1,1,3,0,0,0,1)$ & $(0,3 / 2) \oplus(0,5 / 2)$ \\
\hline$(0,0,1,2,0,0,0,0,1)$ & $(0,3 / 2) \oplus(0,5 / 2)$ & $(0,0,1,2,0,0,0,1,1)$ & $(0,1 / 2) \oplus 2(0,3 / 2) \oplus(0,5 / 2)$ \\
\hline$(0,0,1,2,0,0,0,2,1)$ & $2(0,1 / 2) \oplus 2(0,3 / 2) \oplus(0,5 / 2)$ & $(0,0,1,2,1,0,0,0,1)$ & $(0,1 / 2) \oplus 2(0,3 / 2) \oplus(0,5 / 2)$ \\
\hline$(0,0,1,2,1,0,0,1,1)$ & $4(0,1 / 2) \oplus 4(0,3 / 2) \oplus(0,5 / 2)$ & $(0,0,1,2,1,1,0,0,1)$ & $(0,1 / 2) \oplus 2(0,3 / 2) \oplus(0,5 / 2)$ \\
\hline$(0,0,1,2,2,0,0,0,1)$ & $2(0,1 / 2) \oplus 2(0,3 / 2) \oplus(0,5 / 2)$ & $(0,0,1,3,0,0,0,0,1)$ & $(0,5 / 2) \oplus(0,7 / 2)$ \\
\hline$(0,0,1,3,0,0,0,1,1)$ & $(0,3 / 2) \oplus 2(0,5 / 2) \oplus(0,7 / 2)$ & $(0,0,1,3,1,0,0,0,1)$ & $(0,3 / 2) \oplus 2(0,5 / 2) \oplus(0,7 / 2)$ \\
\hline$(0,0,1,4,0,0,0,0,1)$ & $(0,7 / 2) \oplus(0,9 / 2)$ & $(0,0,2,0,0,0,0,0,1)$ & $(0,3 / 2)$ \\
\hline$(0,0,2,1,0,0,0,0,1)$ & $(0,1 / 2) \oplus(0,3 / 2)$ & $(0,0,2,1,0,0,0,1,1)$ & $(0,1 / 2) \oplus(0,3 / 2)$ \\
\hline$(0,0,2,1,1,0,0,0,1)$ & $(0,1 / 2) \oplus(0,3 / 2)$ & $(0,0,2,1,1,0,0,1,1)$ & $(0,1 / 2) \oplus(0,3 / 2)$ \\
\hline$(0,0,2,1,1,1,0,0,1)$ & $(0,1 / 2) \oplus(0,3 / 2)$ & $(0,0,2,2,0,0,0,0,1)$ & $(0,1 / 2) \oplus(0,3 / 2) \oplus(0,5 / 2)$ \\
\hline$(0,0,2,2,0,0,0,1,1)$ & $2(0,1 / 2) \oplus 2(0,3 / 2) \oplus(0,5 / 2)$ & $(0,0,2,2,1,0,0,0,1)$ & $2(0,1 / 2) \oplus 2(0,3 / 2) \oplus(0,5 / 2)$ \\
\hline$(0,0,2,3,0,0,0,0,1)$ & $(0,3 / 2) \oplus(0,5 / 2) \oplus(0,7 / 2)$ & $(0,0,3,0,0,0,0,0,1)$ & $(0,5 / 2)$ \\
\hline$(0,0,3,1,0,0,0,0,1)$ & $(0,3 / 2) \oplus(0,5 / 2)$ & $(0,0,3,1,0,0,0,1,1)$ & $(0,3 / 2) \oplus(0,5 / 2)$ \\
\hline$(0,0,3,1,1,0,0,0,1)$ & $(0,3 / 2) \oplus(0,5 / 2)$ & $(0,0,3,2,0,0,0,0,1)$ & $(0,1 / 2) \oplus(0,3 / 2) \oplus(0,5 / 2)$ \\
\hline$(0,0,4,0,0,0,0,0,1)$ & $(0,7 / 2)$ & $(0,0,4,1,0,0,0,0,1)$ & $(0,5 / 2) \oplus(0,7 / 2)$ \\
\hline$(0,0,5,0,0,0,0,0,1)$ & $(0,9 / 2)$ & $(0,1,1,0,0,0,0,0,1)$ & $(0,1 / 2)$ \\
\hline$(0,1,1,1,0,0,0,0,1)$ & $(0,1 / 2) \oplus(0,3 / 2)$ & $(0,1,1,1,0,0,0,1,1)$ & $2(0,1 / 2) \oplus(0,3 / 2)$ \\
\hline$(0,1,1,1,0,0,0,2,1)$ & $(0,1 / 2) \oplus(0,3 / 2)$ & $(0,1,1,1,1,0,0,0,1)$ & $2(0,1 / 2) \oplus(0,3 / 2)$ \\
\hline$(0,1,1,1,1,0,0,1,1)$ & $3(0,1 / 2) \oplus(0,3 / 2)$ & $(0,1,1,1,1,1,0,0,1)$ & $2(0,1 / 2) \oplus(0,3 / 2)$ \\
\hline$(0,1,1,1,2,0,0,0,1)$ & $(0,1 / 2) \oplus(0,3 / 2)$ & $(0,1,1,2,0,0,0,0,1)$ & $(0,3 / 2) \oplus(0,5 / 2)$ \\
\hline$(0,1,1,2,0,0,0,1,1)$ & $(0,1 / 2) \oplus 2(0,3 / 2) \oplus(0,5 / 2)$ & $(0,1,1,2,1,0,0,0,1)$ & $(0,1 / 2) \oplus 2(0,3 / 2) \oplus(0,5 / 2)$ \\
\hline$(0,1,1,3,0,0,0,0,1)$ & $(0,5 / 2) \oplus(0,7 / 2)$ & $(0,1,2,0,0,0,0,0,1)$ & $(0,1 / 2) \oplus(0,3 / 2)$ \\
\hline$(0,1,2,1,0,0,0,0,1)$ & $2(0,1 / 2) \oplus(0,3 / 2)$ & $(0,1,2,1,0,0,0,1,1)$ & $2(0,1 / 2) \oplus(0,3 / 2)$ \\
\hline$(0,1,2,1,1,0,0,0,1)$ & $2(0,1 / 2) \oplus(0,3 / 2)$ & $(0,1,2,2,0,0,0,0,1)$ & $(0,1 / 2) \oplus 2(0,3 / 2) \oplus(0,5 / 2)$ \\
\hline$(0,1,3,0,0,0,0,0,1)$ & $(0,3 / 2) \oplus(0,5 / 2)$ & $(0,1,3,1,0,0,0,0,1)$ & $(0,1 / 2) \oplus 2(0,3 / 2) \oplus(0,5 / 2)$ \\
\hline$(0,1,4,0,0,0,0,0,1)$ & $(0,5 / 2) \oplus(0,7 / 2)$ & $(0,2,2,0,0,0,0,0,1)$ & $(0,1 / 2) \oplus(0,3 / 2)$ \\
\hline$(0,2,2,1,0,0,0,0,1)$ & $(0,1 / 2) \oplus(0,3 / 2)$ & $(0,2,3,0,0,0,0,0,1)$ & $(0,1 / 2) \oplus(0,3 / 2) \oplus(0,5 / 2)$ \\
\hline$(0,3,2,0,0,0,0,0,1)$ & $(0,3 / 2)$ & $(1,1,1,0,0,0,0,0,1)$ & $(0,1 / 2)$ \\
\hline$(1,1,1,1,0,0,0,0,1)$ & $(0,1 / 2) \oplus(0,3 / 2)$ & $(1,1,1,1,0,0,0,1,1)$ & $2(0,1 / 2) \oplus(0,3 / 2)$ \\
\hline$(1,1,1,1,1,0,0,0,1)$ & $2(0,1 / 2) \oplus(0,3 / 2)$ & $(1,1,1,2,0,0,0,0,1)$ & $(0,3 / 2) \oplus(0,5 / 2)$ \\
\hline$(1,1,2,0,0,0,0,0,1)$ & $(0,1 / 2) \oplus(0,3 / 2)$ & $(1,1,2,1,0,0,0,0,1)$ & $2(0,1 / 2) \oplus(0,3 / 2)$ \\
\hline$(1,1,3,0,0,0,0,0,1)$ & $(0,3 / 2) \oplus(0,5 / 2)$ & $(1,2,2,0,0,0,0,0,1)$ & $2(0,1 / 2) \oplus(0,3 / 2)$ \\
\hline$(0,0,0,0,0,0,0,3,2)$ & $(0,5 / 2)$ & $(0,0,0,0,0,0,0,4,2)$ & $(0,5 / 2) \oplus(0,7 / 2) \oplus(1 / 2,4)$ \\
\hline$(0,0,0,0,3,0,0,0,2)$ & $(0,5 / 2)$ & $(0,0,0,0,3,1,0,0,2)$ & $(0,3 / 2) \oplus(0,5 / 2)$ \\
\hline$(0,0,0,0,4,0,0,0,2)$ & $(0,5 / 2) \oplus(0,7 / 2) \oplus(1 / 2,4)$ & $(0,0,0,1,0,0,0,0,2)$ & $(0,5 / 2)$ \\
\hline
\end{tabular}




\begin{tabular}{|c|c|c|c|}
\hline$\beta$ & $\oplus N_{j_{l}, j_{r}}^{\beta}\left(j_{l}, j_{r}\right)$ & $\beta$ & $\oplus N_{j_{l}, j_{r}}^{\beta}\left(j_{l}, j_{r}\right)$ \\
\hline$(0,0,0,1,0,0,0,1,2)$ & $(0,3 / 2) \oplus(0,5 / 2)$ & $(0,0,0,1,0,0,0,2,2)$ & $(0,1 / 2) \oplus(0,3 / 2) \oplus(0,5 / 2)$ \\
\hline$(0,0,0,1,0,0,0,3,2)$ & $\begin{array}{l}(0,1 / 2) \oplus 2(0,3 / 2) \oplus 2(0,5 / 2) \oplus \\
(0,7 / 2)\end{array}$ & $(0,0,0,1,1,0,0,0,2)$ & $(0,3 / 2) \oplus(0,5 / 2)$ \\
\hline$(0,0,0,1,1,0,0,1,2)$ & $(0,1 / 2) \oplus 2(0,3 / 2) \oplus(0,5 / 2)$ & $(0,0,0,1,1,0,0,2,2)$ & $2(0,1 / 2) \oplus 2(0,3 / 2) \oplus(0,5 / 2)$ \\
\hline$(0,0,0,1,1,1,0,0,2)$ & $(0,3 / 2) \oplus(0,5 / 2)$ & $(0,0,0,1,1,1,0,1,2)$ & $(0,1 / 2) \oplus 2(0,3 / 2) \oplus(0,5 / 2)$ \\
\hline$(0,0,0,1,1,1,1,0,2)$ & $(0,3 / 2) \oplus(0,5 / 2)$ & $(0,0,0,1,2,0,0,0,2)$ & $(0,1 / 2) \oplus(0,3 / 2) \oplus(0,5 / 2)$ \\
\hline$(0,0,0,1,2,0,0,1,2)$ & $2(0,1 / 2) \oplus 2(0,3 / 2) \oplus(0,5 / 2)$ & $(0,0,0,1,2,1,0,0,2)$ & $(0,1 / 2) \oplus 2(0,3 / 2) \oplus(0,5 / 2)$ \\
\hline$(0,0,0,1,3,0,0,0,2)$ & $\begin{array}{l}(0,1 / 2) \oplus 2(0,3 / 2) \oplus 2(0,5 / 2) \oplus \\
(0,7 / 2)\end{array}$ & $(0,0,0,2,0,0,0,0,2)$ & $(0,5 / 2) \oplus(0,7 / 2) \oplus(1 / 2,4)$ \\
\hline$(0,0,0,2,0,0,0,1,2)$ & $\begin{array}{l}(0,3 / 2) \oplus 3(0,5 / 2) \oplus 2(0,7 / 2) \oplus \\
(1 / 2,3) \oplus(1 / 2,4)\end{array}$ & $(0,0,0,2,0,0,0,2,2)$ & $\begin{array}{l}(0,1 / 2) \oplus 3(0,3 / 2) \oplus 4(0,5 / 2) \oplus \\
2(0,7 / 2) \oplus(1 / 2,2) \oplus(1 / 2,3) \oplus \\
(1 / 2,4)\end{array}$ \\
\hline$(0,0,0,2,1,0,0,0,2)$ & $\begin{array}{l}(0,3 / 2) \oplus 3(0,5 / 2) \oplus 2(0,7 / 2) \oplus \\
(1 / 2,3) \oplus(1 / 2,4)\end{array}$ & $(0,0,0,2,1,0,0,1,2)$ & $\begin{array}{l}(0,1 / 2) \oplus 5(0,3 / 2) \oplus 7(0,5 / 2) \oplus \\
3(0,7 / 2) \oplus(1 / 2,2) \oplus 2(1 / 2,3) \oplus \\
(1 / 2,4)\end{array}$ \\
\hline$(0,0,0,2,1,1,0,0,2)$ & $\begin{array}{l}(0,3 / 2) \oplus 3(0,5 / 2) \oplus 2(0,7 / 2) \oplus \\
(1 / 2,3) \oplus(1 / 2,4)\end{array}$ & $(0,0,0,2,2,0,0,0,2)$ & $\begin{array}{l}(0,1 / 2) \oplus 3(0,3 / 2) \oplus 4(0,5 / 2) \oplus \\
2(0,7 / 2) \oplus(1 / 2,2) \oplus(1 / 2,3) \oplus \\
(1 / 2,4)\end{array}$ \\
\hline$(0,0,0,3,0,0,0,0,2)$ & $\begin{array}{l}(0,5 / 2) \oplus(0,7 / 2) \oplus 2(0,9 / 2) \oplus \\
(1 / 2,4) \oplus(1 / 2,5) \oplus(1,11 / 2)\end{array}$ & $(0,0,0,3,0,0,0,1,2)$ & $\begin{array}{l}(0,3 / 2) \oplus 3(0,5 / 2) \oplus 5(0,7 / 2) \oplus \\
3(0,9 / 2) \oplus(1 / 2,3) \oplus 3(1 / 2,4) \oplus \\
2(1 / 2,5) \oplus(1,9 / 2) \oplus(1,11 / 2)\end{array}$ \\
\hline$(0,0,0,3,1,0,0,0,2)$ & $\begin{array}{l}(0,3 / 2) \oplus 3(0,5 / 2) \oplus 5(0,7 / 2) \oplus \\
3(0,9 / 2) \oplus(1 / 2,3) \oplus 3(1 / 2,4) \oplus \\
2(1 / 2,5) \oplus(1,9 / 2) \oplus(1,11 / 2)\end{array}$ & $(0,0,0,4,0,0,0,0,2)$ & $\begin{array}{l}(0,5 / 2) \oplus(0,7 / 2) \oplus 2(0,9 / 2) \oplus \\
2(0,11 / 2) \oplus(1 / 2,4) \oplus \\
(1 / 2,5) \oplus 2(1 / 2,6) \oplus(1,11 / 2) \oplus \\
(1,13 / 2) \oplus(3 / 2,7)\end{array}$ \\
\hline$(0,0,1,1,0,0,0,0,2)$ & $(0,3 / 2) \oplus(0,5 / 2)$ & $(0,0,1,1,0,0,0,1,2)$ & $(0,1 / 2) \oplus 2(0,3 / 2) \oplus(0,5 / 2)$ \\
\hline$(0,0,1,1,0,0,0,2,2)$ & $2(0,1 / 2) \oplus 2(0,3 / 2) \oplus(0,5 / 2)$ & $(0,0,1,1,1,0,0,0,2)$ & $(0,1 / 2) \oplus 2(0,3 / 2) \oplus(0,5 / 2)$ \\
\hline$(0,0,1,1,1,0,0,1,2)$ & $3(0,1 / 2) \oplus 3(0,3 / 2) \oplus(0,5 / 2)$ & $(0,0,1,1,1,1,0,0,2)$ & $(0,1 / 2) \oplus 2(0,3 / 2) \oplus(0,5 / 2)$ \\
\hline$(0,0,1,1,2,0,0,0,2)$ & $2(0,1 / 2) \oplus 2(0,3 / 2) \oplus(0,5 / 2)$ & $(0,0,1,2,0,0,0,0,2)$ & $\begin{array}{l}(0,3 / 2) \oplus 3(0,5 / 2) \oplus 2(0,7 / 2) \oplus \\
(1 / 2,3) \oplus(1 / 2,4)\end{array}$ \\
\hline$(0,0,1,2,0,0,0,1,2)$ & $\begin{array}{l}(0,1 / 2) \oplus 5(0,3 / 2) \oplus 7(0,5 / 2) \oplus \\
3(0,7 / 2) \oplus(1 / 2,2) \oplus 2(1 / 2,3) \oplus \\
(1 / 2,4)\end{array}$ & $(0,0,1,2,1,0,0,0,2)$ & $\begin{array}{l}(0,1 / 2) \oplus 5(0,3 / 2) \oplus 7(0,5 / 2) \oplus \\
3(0,7 / 2) \oplus(1 / 2,2) \oplus 2(1 / 2,3) \oplus \\
(1 / 2,4)\end{array}$ \\
\hline$(0,0,1,3,0,0,0,0,2)$ & $\begin{array}{l}(0,3 / 2) \oplus 3(0,5 / 2) \oplus 5(0,7 / 2) \oplus \\
3(0,9 / 2) \oplus(1 / 2,3) \oplus 3(1 / 2,4) \oplus \\
2(1 / 2,5) \oplus(1,9 / 2) \oplus(1,11 / 2)\end{array}$ & $(0,0,2,1,0,0,0,0,2)$ & $(0,1 / 2) \oplus(0,3 / 2) \oplus(0,5 / 2)$ \\
\hline$(0,0,2,1,0,0,0,1,2)$ & $2(0,1 / 2) \oplus 2(0,3 / 2) \oplus(0,5 / 2)$ & $(0,0,2,1,1,0,0,0,2)$ & $2(0,1 / 2) \oplus 2(0,3 / 2) \oplus(0,5 / 2)$ \\
\hline$(0,0,2,2,0,0,0,0,2)$ & $\begin{array}{l}(0,1 / 2) \oplus 3(0,3 / 2) \oplus 4(0,5 / 2) \oplus \\
2(0,7 / 2) \oplus(1 / 2,2) \oplus(1 / 2,3) \oplus \\
(1 / 2,4)\end{array}$ & $(0,0,3,0,0,0,0,0,2)$ & $(0,5 / 2)$ \\
\hline$(0,0,3,1,0,0,0,0,2)$ & $\begin{array}{l}(0,1 / 2) \oplus 2(0,3 / 2) \oplus 2(0,5 / 2) \oplus \\
(0,7 / 2)\end{array}$ & $(0,0,4,0,0,0,0,0,2)$ & $(0,5 / 2) \oplus(0,7 / 2) \oplus(1 / 2,4)$ \\
\hline$(0,1,1,1,0,0,0,0,2)$ & $(0,3 / 2) \oplus(0,5 / 2)$ & $(0,1,1,1,0,0,0,1,2)$ & $(0,1 / 2) \oplus 2(0,3 / 2) \oplus(0,5 / 2)$ \\
\hline$(0,1,1,1,1,0,0,0,2)$ & $(0,1 / 2) \oplus 2(0,3 / 2) \oplus(0,5 / 2)$ & $(0,1,1,2,0,0,0,0,2)$ & $\begin{array}{l}(0,3 / 2) \oplus 3(0,5 / 2) \oplus 2(0,7 / 2) \oplus \\
(1 / 2,3) \oplus(1 / 2,4)\end{array}$ \\
\hline$(0,1,2,1,0,0,0,0,2)$ & $(0,1 / 2) \oplus 2(0,3 / 2) \oplus(0,5 / 2)$ & $(0,1,3,0,0,0,0,0,2)$ & $(0,3 / 2) \oplus(0,5 / 2)$ \\
\hline$(1,1,1,1,0,0,0,0,2)$ & $(0,3 / 2) \oplus(0,5 / 2)$ & $(0,0,0,1,0,0,0,0,3)$ & $(0,7 / 2)$ \\
\hline$(0,0,0,1,0,0,0,1,3)$ & $(0,5 / 2) \oplus(0,7 / 2)$ & $(0,0,0,1,0,0,0,2,3)$ & $(0,3 / 2) \oplus(0,5 / 2) \oplus(0,7 / 2)$ \\
\hline$(0,0,0,1,1,0,0,0,3)$ & $(0,5 / 2) \oplus(0,7 / 2)$ & $(0,0,0,1,1,0,0,1,3)$ & $(0,3 / 2) \oplus 2(0,5 / 2) \oplus(0,7 / 2)$ \\
\hline
\end{tabular}




\begin{tabular}{|c|l|l|l|}
\hline$\beta$ & \multicolumn{1}{|c|}{$\oplus N_{j_{l}, j_{r}}^{\beta}\left(j_{l}, j_{r}\right)$} & \multicolumn{1}{|c|}{$\beta$} & \multicolumn{1}{|c|}{$\oplus N_{j_{l}, j_{r}}^{\beta}\left(j_{l}, j_{r}\right)$} \\
\hline$(0,0,0,1,1,1,0,0,3)$ & $(0,5 / 2) \oplus(0,7 / 2)$ & $(0,0,0,1,2,0,0,0,3)$ & $(0,3 / 2) \oplus(0,5 / 2) \oplus(0,7 / 2)$ \\
\hline$(0,0,0,2,0,0,0,0,3)$ & $(0,5 / 2) \oplus(0,7 / 2) \oplus 2(0,9 / 2) \oplus$ & $(0,0,0,2,0,0,0,1,3)$ & $(0,3 / 2) \oplus 3(0,5 / 2) \oplus 5(0,7 / 2) \oplus$ \\
& $(1 / 2,4) \oplus(1 / 2,5) \oplus(1,11 / 2)$ & & $3(0,9 / 2) \oplus(1 / 2,3) \oplus 3(1 / 2,4) \oplus$ \\
& & & $2(1 / 2,5) \oplus(1,9 / 2) \oplus(1,11 / 2)$ \\
\hline$(0,0,0,2,1,0,0,0,3)$ & $(0,3 / 2) \oplus 3(0,5 / 2) \oplus 5(0,7 / 2) \oplus$ & $(0,0,0,3,0,0,0,0,3)$ & $(0,3 / 2) \oplus(0,5 / 2) \oplus 3(0,7 / 2) \oplus$ \\
& $3(0,9 / 2) \oplus(1 / 2,3) \oplus 3(1 / 2,4) \oplus$ & & $3(0,9 / 2) \oplus 4(0,11 / 2) \oplus$ \\
& $2(1 / 2,5) \oplus(1,9 / 2) \oplus(1,11 / 2)$ & & $(1 / 2,3) \oplus 2(1 / 2,4) \oplus 3(1 / 2,5) \oplus$ \\
& & & $3(1 / 2,6) \oplus(1 / 2,7) \oplus(1,9 / 2) \oplus$ \\
& & $(0,0,1,1,0,0,0,1,3)$ & $(0,3 / 2) \oplus 2(0,5 / 2) \oplus(0,7 / 2)$ \\
\hline$(0,0,1,1,0,0,0,0,3)$ & $(0,5 / 2) \oplus(0,7 / 2)$ & $(0,0,1,2,0,0,0,0,3)$ & $(0,3 / 2) \oplus 3(0,5 / 2) \oplus 5(0,7 / 2) \oplus$ \\
\hline$(0,0,1,1,1,0,0,0,3)$ & $(0,3 / 2) \oplus 2(0,5 / 2) \oplus(0,7 / 2)$ & & $3(0,9 / 2) \oplus(1 / 2,3) \oplus 3(1 / 2,4) \oplus$ \\
& & & $2(1 / 2,5) \oplus(1,9 / 2) \oplus(1,11 / 2)$ \\
\hline$(0,0,2,1,0,0,0,0,3)$ & $(0,3 / 2) \oplus(0,5 / 2) \oplus(0,7 / 2)$ & $(0,1,1,1,0,0,0,0,3)$ & $(0,5 / 2) \oplus(0,7 / 2)$ \\
\hline$(0,0,0,1,0,0,0,0,4)$ & $(0,9 / 2)$ & $(0,0,0,1,0,0,0,1,4)$ & $(0,7 / 2) \oplus(0,9 / 2)$ \\
\hline$(0,0,0,1,1,0,0,0,4)$ & $(0,7 / 2) \oplus(0,9 / 2)$ & $(0,0,0,2,0,0,0,0,4)$ & $(0,5 / 2) \oplus(0,7 / 2) \oplus 2(0,9 / 2) \oplus$ \\
& & & $2(0,11 / 2) \oplus(1 / 2,4) \oplus$ \\
& & & $(1 / 2,5) \oplus 2(1 / 2,6) \oplus(1,11 / 2) \oplus$ \\
\hline$(0,0,1,1,0,0,0,0,4)$ & $(0,7 / 2) \oplus(0,9 / 2)$ & $(0,0,0,1,0,0,0,0,5)$ & $(0,11 / 2)$ \\
\hline
\end{tabular}

Table 26. Refined BPS invariants of $6 \mathrm{~d} E_{7}$ minimal SCFT.

\begin{tabular}{|l|l|l|l|}
\hline \multicolumn{1}{|c|}{$\beta$} & \multicolumn{1}{|c|}{$\oplus N_{j_{l}, j_{r}}^{\beta}\left(j_{l}, j_{r}\right)$} & \multicolumn{1}{|c|}{$\oplus N_{j_{l}, j_{r}}^{\beta}\left(j_{l}, j_{r}\right)$} \\
\hline$(0,0,0,0,0,0,0,0,0,1)$ & $(0,1 / 2)$ & $(0,0,0,0,0,0,0,0,1,1)$ & $(0,1 / 2)$ \\
\hline$(0,0,0,0,0,0,0,0,2,1)$ & $(0,3 / 2)$ & $(0,0,0,0,0,0,0,0,3,1)$ & $(0,5 / 2)$ \\
\hline$(0,0,0,0,0,0,0,0,4,1)$ & $(0,7 / 2)$ & $(0,0,0,0,0,0,0,0,5,1)$ & $(0,9 / 2)$ \\
\hline$(0,0,0,0,0,0,1,0,0,1)$ & $(0,1 / 2)$ & $(0,0,0,0,0,0,1,1,0,1)$ & $(0,1 / 2)$ \\
\hline$(0,0,0,0,0,0,2,0,0,1)$ & $(0,3 / 2)$ & $(0,0,0,0,0,0,2,1,0,1)$ & $(0,1 / 2) \oplus(0,3 / 2)$ \\
\hline$(0,0,0,0,0,0,2,2,0,1)$ & $(0,1 / 2) \oplus(0,3 / 2)$ & $(0,0,0,0,0,0,2,3,0,1)$ & $(0,3 / 2)$ \\
\hline$(0,0,0,0,0,0,3,0,0,1)$ & $(0,5 / 2)$ & $(0,0,0,0,0,0,3,1,0,1)$ & $(0,3 / 2) \oplus(0,5 / 2)$ \\
\hline$(0,0,0,0,0,0,3,2,0,1)$ & $(0,1 / 2) \oplus(0,3 / 2) \oplus(0,5 / 2)$ & $(0,0,0,0,0,0,4,0,0,1)$ & $(0,7 / 2)$ \\
\hline$(0,0,0,0,0,0,4,1,0,1)$ & $(0,5 / 2) \oplus(0,7 / 2)$ & $(0,0,0,0,0,0,5,0,0,1)$ & $(0,9 / 2)$ \\
\hline$(0,0,0,0,0,1,0,0,0,1)$ & $(0,3 / 2)$ & $(0,0,0,0,0,1,0,0,1,1)$ & $(0,1 / 2) \oplus(0,3 / 2)$ \\
\hline$(0,0,0,0,0,1,0,0,2,1)$ & $(0,1 / 2) \oplus(0,3 / 2)$ & $(0,0,0,0,0,1,0,0,3,1)$ & $(0,3 / 2) \oplus(0,5 / 2)$ \\
\hline$(0,0,0,0,0,1,0,0,4,1)$ & $(0,5 / 2) \oplus(0,7 / 2)$ & $(0,0,0,0,0,1,1,0,0,1)$ & $(0,1 / 2) \oplus(0,3 / 2)$ \\
\hline$(0,0,0,0,0,1,1,0,1,1)$ & $2(0,1 / 2) \oplus(0,3 / 2)$ & $(0,0,0,0,0,1,1,0,2,1)$ & $(0,1 / 2) \oplus(0,3 / 2)$ \\
\hline$(0,0,0,0,0,1,1,0,3,1)$ & $(0,3 / 2) \oplus(0,5 / 2)$ & $(0,0,0,0,0,1,1,1,0,1)$ & $(0,1 / 2) \oplus(0,3 / 2)$ \\
\hline$(0,0,0,0,0,1,1,1,1,1)$ & $2(0,1 / 2) \oplus(0,3 / 2)$ & $(0,0,0,0,0,1,1,1,2,1)$ & $(0,1 / 2) \oplus(0,3 / 2)$ \\
\hline$(0,0,0,0,0,1,2,0,0,1)$ & $(0,1 / 2) \oplus(0,3 / 2)$ & $(0,0,0,0,0,1,2,0,1,1)$ & $(0,1 / 2) \oplus(0,3 / 2)$ \\
\hline$(0,0,0,0,0,1,2,1,0,1)$ & $2(0,1 / 2) \oplus(0,3 / 2)$ & $(0,0,0,0,0,1,2,1,1,1)$ & $2(0,1 / 2) \oplus(0,3 / 2)$ \\
\hline$(0,0,0,0,0,1,2,2,0,1)$ & $(0,1 / 2) \oplus(0,3 / 2)$ & $(0,0,0,0,0,1,3,0,0,1)$ & $(0,3 / 2) \oplus(0,5 / 2)$ \\
\hline$(0,0,0,0,0,1,3,0,1,1)$ & $(0,3 / 2) \oplus(0,5 / 2)$ & $(0,0,0,0,0,1,3,1,0,1)$ & $(0,1 / 2) \oplus 2(0,3 / 2) \oplus(0,5 / 2)$ \\
\hline$(0,0,0,0,0,1,4,0,0,1)$ & $(0,5 / 2) \oplus(0,7 / 2)$ & $(0,0,0,0,0,2,0,0,0,1)$ & $(0,5 / 2)$ \\
\hline$(0,0,0,0,0,2,0,0,1,1)$ & $(0,3 / 2) \oplus(0,5 / 2)$ & $(0,0,0,0,0,2,0,0,2,1)$ & $(0,1 / 2) \oplus(0,3 / 2) \oplus(0,5 / 2)$ \\
\hline$(0,0,0,0,0,2,0,0,3,1)$ & $(0,1 / 2) \oplus(0,3 / 2) \oplus(0,5 / 2)$ & $(0,0,0,0,0,2,1,0,0,1)$ & $(0,3 / 2) \oplus(0,5 / 2)$ \\
\hline$(0,0,0,0,0,2,1,0,1,1)$ & $(0,1 / 2) \oplus 2(0,3 / 2) \oplus(0,5 / 2)$ & $(0,0,0,0,0,2,1,0,2,1)$ & $2(0,1 / 2) \oplus 2(0,3 / 2) \oplus(0,5 / 2)$ \\
\hline$(0,0,0,0,0,2,1,1,0,1)$ & $(0,3 / 2) \oplus(0,5 / 2)$ & $(0,0,0,0,0,2,1,1,1,1)$ & $(0,1 / 2) \oplus 2(0,3 / 2) \oplus(0,5 / 2)$ \\
\hline
\end{tabular}




\begin{tabular}{|c|c|c|c|}
\hline$\beta$ & $\oplus N_{j_{l}, j_{r}}^{\beta}\left(j_{l}, j_{r}\right)$ & $\beta$ & $\oplus N_{j_{l}, j_{r}}^{\beta}\left(j_{l}, j_{r}\right)$ \\
\hline$(0,0,0,0,0,2,2,0,0,1)$ & \begin{tabular}{|l}
$(0,1 / 2) \oplus(0,3 / 2) \oplus(0,5 / 2)$ \\
\end{tabular} & \begin{tabular}{|l|l|}
$(0,0,0,0,0,2,2,0,1,1)$ \\
\end{tabular} & $2(0,1 / 2) \oplus 2(0,3 / 2) \oplus(0,5 / 2)$ \\
\hline$(0,0,0,0,0,2,2,1,0,1)$ & 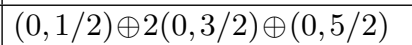 & \begin{tabular}{|l|l|}
$(0,0,0,0,0,2,3,0,0,1)$ \\
\end{tabular} & $(0,1 / 2) \oplus(0,3 / 2) \oplus(0,5 / 2)$ \\
\hline$(0,0,0,0,0,3,0,0,0,1)$ & $(0,7 / 2)$ & $(0,0,0,0,0,3,0,0,1,1)$ & $(0,5 / 2) \oplus(0,7 / 2)$ \\
\hline$(0,0,0,0,0,3,0,0,2,1)$ & $(0,3 / 2) \oplus(0,5 / 2) \oplus(0,7 / 2)$ & $(0,0,0,0,0,3,1,0,0,1)$ & $(0,5 / 2) \oplus(0,7 / 2)$ \\
\hline$(0,0,0,0,0,3,1,0,1,1)$ & $\mid(0,3 / 2) \oplus 2(0,5 / 2) \oplus(0,7 / 2)$ & $(0,0,0,0,0,3,1,1,0,1)$ & $(0,5 / 2) \oplus(0,7 / 2)$ \\
\hline$(0,0,0,0,0,3,2,0,0,1)$ & $(0,3 / 2) \oplus(0,5 / 2) \oplus(0,7 / 2)$ & $(0,0,0,0,0,4,0,0,0,1)$ & $(0,9 / 2)$ \\
\hline$(0,0,0,0,0,4,0,0,1,1)$ & $(0,7 / 2) \oplus(0,9 / 2)$ & $(0,0,0,0,0,4,1,0,0,1)$ & $(0,7 / 2) \oplus(0,9 / 2)$ \\
\hline$(0,0,0,0,0,5,0,0,0,1)$ & $(0,11 / 2)$ & $(0,0,0,0,1,0,0,0,0,1)$ & $(0,1 / 2)$ \\
\hline$(0,0,0,0,1,1,0,0,0,1)$ & $(0,1 / 2) \oplus(0,3 / 2)$ & $(0,0,0,0,1,1,0,0,1,1)$ & $2(0,1 / 2) \oplus(0,3 / 2)$ \\
\hline$(0,0,0,0,1,1,0,0,2,1)$ & $(0,1 / 2) \oplus(0,3 / 2)$ & $\mid(0,0,0,0,1,1,0,0,3,1)$ & $(0,3 / 2) \oplus(0,5 / 2)$ \\
\hline$(0,0,0,0,1,1,1,0,0,1)$ & $2(0,1 / 2) \oplus(0,3 / 2)$ & $(0,0,0,0,1,1,1,0,1,1)$ & $3(0,1 / 2) \oplus(0,3 / 2)$ \\
\hline$(0,0,0,0,1,1,1,0,2,1)$ & $(0,1 / 2) \oplus(0,3 / 2)$ & $(0,0,0,0,1,1,1,1,0,1)$ & $2(0,1 / 2) \oplus(0,3 / 2)$ \\
\hline$(0,0,0,0,1,1,1,1,1,1)$ & $3(0,1 / 2) \oplus(0,3 / 2)$ & $(0,0,0,0,1,1,2,0,0,1)$ & $(0,1 / 2) \oplus(0,3 / 2)$ \\
\hline$(0,0,0,0,1,1,2,0,1,1)$ & $(0,1 / 2) \oplus(0,3 / 2)$ & $(0,0,0,0,1,1,2,1,0,1)$ & $2(0,1 / 2) \oplus(0,3 / 2)$ \\
\hline$(0,0,0,0,1,1,3,0,0,1)$ & $(0,3 / 2) \oplus(0,5 / 2)$ & $(0,0,0,0,1,2,0,0,0,1)$ & $(0,3 / 2) \oplus(0,5 / 2)$ \\
\hline$(0,0,0,0,1,2,0,0,1,1)$ & $(0,1 / 2) \oplus 2(0,3 / 2) \oplus(0,5 / 2)$ & $(0,0,0,0,1,2,0,0,2,1)$ & $2(0,1 / 2) \oplus 2(0,3 / 2) \oplus(0,5 / 2)$ \\
\hline$(0,0,0,0,1,2,1,0,0,1)$ & $(0,1 / 2) \oplus 2(0,3 / 2) \oplus(0,5 / 2)$ & $(0,0,0,0,1,2,1,0,1,1)$ & $4(0,1 / 2) \oplus 4(0,3 / 2) \oplus(0,5 / 2)$ \\
\hline$(0,0,0,0,1,2,1,1,0,1)$ & 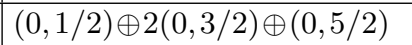 & \begin{tabular}{|l|l|}
$(0,0,0,0,1,2,2,0,0,1)$ \\
\end{tabular} & $2(0,1 / 2) \oplus 2(0,3 / 2) \oplus(0,5 / 2)$ \\
\hline$(0,0,0,0,1,3,0,0,0,1)$ & $(0,5 / 2) \oplus(0,7 / 2)$ & $(0,0,0,0,1,3,0,0,1,1)$ & $(0,3 / 2) \oplus 2(0,5 / 2) \oplus(0,7 / 2)$ \\
\hline$(0,0,0,0,1,3,1,0,0,1)$ & $\mid(0,3 / 2) \oplus 2(0,5 / 2) \oplus(0,7 / 2)$ & $(0,0,0,0,1,4,0,0,0,1)$ & $(0,7 / 2) \oplus(0,9 / 2)$ \\
\hline$(0,0,0,0,2,0,0,0,0,1)$ & $(0,3 / 2)$ & $(0,0,0,0,2,1,0,0,0,1)$ & $(0,1 / 2) \oplus(0,3 / 2)$ \\
\hline$(0,0,0,0,2,1,0,0,1,1)$ & $(0,1 / 2) \oplus(0,3 / 2)$ & $(0,0,0,0,2,1,1,0,0,1)$ & $(0,1 / 2) \oplus(0,3 / 2)$ \\
\hline$(0,0,0,0,2,1,1,0,1,1)$ & $(0,1 / 2) \oplus(0,3 / 2)$ & \begin{tabular}{|l|l|}
$(0,0,0,0,2,1,1,1,0,1)$ \\
\end{tabular} & $(0,1 / 2) \oplus(0,3 / 2)$ \\
\hline$(0,0,0,0,2,2,0,0,0,1)$ & $(0,1 / 2) \oplus(0,3 / 2) \oplus(0,5 / 2)$ & $(0,0,0,0,2,2,0,0,1,1)$ & $2(0,1 / 2) \oplus 2(0,3 / 2) \oplus(0,5 / 2)$ \\
\hline$(0,0,0,0,2,2,1,0,0,1)$ & $2(0,1 / 2) \oplus 2(0,3 / 2) \oplus(0,5 / 2)$ & $(0,0,0,0,2,3,0,0,0,1)$ & $(0,3 / 2) \oplus(0,5 / 2) \oplus(0,7 / 2)$ \\
\hline$(0,0,0,0,3,0,0,0,0,1)$ & $(0,5 / 2)$ & \begin{tabular}{|l|l|}
$(0,0,0,0,3,1,0,0,0,1)$ \\
\end{tabular} & $(0,3 / 2) \oplus(0,5 / 2)$ \\
\hline$(0,0,0,0,3,1,0,0,1,1)$ & $(0,3 / 2) \oplus(0,5 / 2)$ & \begin{tabular}{|l|l|}
$(0,0,0,0,3,1,1,0,0,1)$ \\
\end{tabular} & $(0,3 / 2) \oplus(0,5 / 2)$ \\
\hline$(0,0,0,0,3,2,0,0,0,1)$ & $(0,1 / 2) \oplus(0,3 / 2) \oplus(0,5 / 2)$ & $(0,0,0,0,4,0,0,0,0,1)$ & $(0,7 / 2)$ \\
\hline$(0,0,0,0,4,1,0,0,0,1)$ & $(0,5 / 2) \oplus(0,7 / 2)$ & $(0,0,0,0,5,0,0,0,0,1)$ & $(0,9 / 2)$ \\
\hline$(0,0,0,1,1,0,0,0,0,1)$ & $(0,1 / 2)$ & $(0,0,0,1,1,1,0,0,0,1)$ & $(0,1 / 2) \oplus(0,3 / 2)$ \\
\hline$(0,0,0,1,1,1,0,0,1,1)$ & $2(0,1 / 2) \oplus(0,3 / 2)$ & $(0,0,0,1,1,1,0,0,2,1)$ & $(0,1 / 2) \oplus(0,3 / 2)$ \\
\hline$(0,0,0,1,1,1,1,0,0,1)$ & $2(0,1 / 2) \oplus(0,3 / 2)$ & $(0,0,0,1,1,1,1,0,1,1)$ & $3(0,1 / 2) \oplus(0,3 / 2)$ \\
\hline$(0,0,0,1,1,1,1,1,0,1)$ & $2(0,1 / 2) \oplus(0,3 / 2)$ & $(0,0,0,1,1,1,2,0,0,1)$ & $(0,1 / 2) \oplus(0,3 / 2)$ \\
\hline$(0,0,0,1,1,2,0,0,0,1)$ & $(0,3 / 2) \oplus(0,5 / 2)$ & \begin{tabular}{|l|l|}
$(0,0,0,1,1,2,0,0,1,1)$ \\
\end{tabular} & $(0,1 / 2) \oplus 2(0,3 / 2) \oplus(0,5 / 2)$ \\
\hline$(0,0,0,1,1,2,1,0,0,1)$ & $\mid(0,1 / 2) \oplus 2(0,3 / 2) \oplus(0,5 / 2)$ & $\mid(0,0,0,1,1,3,0,0,0,1)$ & $(0,5 / 2) \oplus(0,7 / 2)$ \\
\hline$(0,0,0,1,2,0,0,0,0,1)$ & $(0,1 / 2) \oplus(0,3 / 2)$ & $(0,0,0,1,2,1,0,0,0,1)$ & $2(0,1 / 2) \oplus(0,3 / 2)$ \\
\hline$(0,0,0,1,2,1,0,0,1,1)$ & $2(0,1 / 2) \oplus(0,3 / 2)$ & $(0,0,0,1,2,1,1,0,0,1)$ & $2(0,1 / 2) \oplus(0,3 / 2)$ \\
\hline$(0,0,0,1,2,2,0,0,0,1)$ & $(0,1 / 2) \oplus 2(0,3 / 2) \oplus(0,5 / 2)$ & $(0,0,0,1,3,0,0,0,0,1)$ & $(0,3 / 2) \oplus(0,5 / 2)$ \\
\hline$(0,0,0,1,3,1,0,0,0,1)$ & $(0,1 / 2) \oplus 2(0,3 / 2) \oplus(0,5 / 2)$ & $\mid(0,0,0,1,4,0,0,0,0,1)$ & $(0,5 / 2) \oplus(0,7 / 2)$ \\
\hline$(0,0,0,2,2,0,0,0,0,1)$ & $(0,1 / 2) \oplus(0,3 / 2)$ & $(0,0,0,2,2,1,0,0,0,1)$ & $(0,1 / 2) \oplus(0,3 / 2)$ \\
\hline$(0,0,0,2,3,0,0,0,0,1)$ & $(0,1 / 2) \oplus(0,3 / 2) \oplus$ & $(0,0,0,3,2,0,0,0,0,1)$ & $(0,3 / 2)$ \\
\hline$(0,0,1,1,1,0,0,0,0,1)$ & $(0,1 / 2)$ & $(0,0,1,1,1,1,0,0,0,1)$ & $(0,1 / 2) \oplus(0,3 / 2)$ \\
\hline$(0,0,1,1,1,1,0,0,1,1)$ & $2(0,1 / 2) \oplus(0,3 / 2)$ & \begin{tabular}{|l|l|}
$(0,0,1,1,1,1,1,0,0,1)$ \\
\end{tabular} & $2(0,1 / 2) \oplus(0,3 / 2)$ \\
\hline$(0,0,1,1,1,2,0,0,0,1)$ & $(0,3 / 2) \oplus(0,5 / 2)$ & $(0,0,1,1,2,0,0,0,0,1)$ & $(0,1 / 2) \oplus(0,3 / 2)$ \\
\hline$(0,0,1,1,2,1,0,0,0,1)$ & $2(0,1 / 2) \oplus(0,3 / 2)$ & $(0,0,1,1,3,0,0,0,0,1)$ & $(0,3 / 2) \oplus(0,5 / 2)$ \\
\hline$(0,0,1,2,2,0,0,0,0,1)$ & $2(0,1 / 2) \oplus(0,3 / 2)$ & $(0,1,1,1,1,0,0,0,0,1)$ & $(0,1 / 2)$ \\
\hline$(0,1,1,1,1,1,0,0,0,1)$ & $(0,1 / 2) \oplus(0,3 / 2)$ & \begin{tabular}{|l|l|}
$(0,1,1,1,2,0,0,0,0,1)$ \\
\end{tabular} & $(0,1 / 2) \oplus(0,3 / 2)$ \\
\hline$(1,1,1,1,1,0,0,0,0,1)$ & $(0,1 / 2)$ & $(0,0,0,0,0,0,0,0,3,2)$ & $(0,5 / 2)$ \\
\hline$(0,0,0,0,0,0,0,0,4,2)$ & $(0,5 / 2) \oplus(0,7 / 2) \oplus(1 / 2,4)$ & $(0,0,0,0,0,0,3,0,0,2)$ & $(0,5 / 2)$ \\
\hline
\end{tabular}




\begin{tabular}{|c|c|c|c|}
\hline$\beta$ & $\oplus N_{j_{l}, j_{r}}^{\beta}\left(j_{l}, j_{r}\right)$ & $\beta$ & $\oplus N_{j_{l}, j_{r}}^{\beta}\left(j_{l}, j_{r}\right)$ \\
\hline$(0,0,0,0,0,0,3,1,0,2)$ & $, 3 / 2) \oplus(0,5 / 2)$ & $0,0,0,0,0,0,4,0,0,2)$ & $(0,5 / 2) \oplus(0,7 / 2) \oplus(1 / 2,4)$ \\
\hline$(0,0,0,0,0,1,0,0,0,2)$ & $0,5 / 2)$ & $(0,0,0,0,0,1,0,0,1,2)$ & $(0,3 / 2) \oplus(0,5 / 2)$ \\
\hline$(0,0,0,0,0,1,0,0,2,2)$ & $0,1 / 2) \oplus(0,3 / 2) \oplus(0$ & $(0,0,0,0,0,1,0,0,3,2)$ & $\begin{array}{l}(0,1 / 2) \oplus 2(0,3 / 2) \oplus 2(0,5 / 2) \oplus \\
(0,7 / 2)\end{array}$ \\
\hline$(0,0,0,0,0,1,1,0,0,2)$ & $0,3 / 2) \oplus(0,5 / 2)$ & $(0,0,0,0,0,1,1,0,1,2)$ & $(0,1 / 2) \oplus 2(0,3 / 2) \oplus(0,5 / 2)$ \\
\hline$(0,0,0,0,0,1,1,0,2,2)$ & $(0,1 / 2) \oplus 2(0,3 / 2) \oplus(0,5 / 2)$ & $(0,0,0,0,0,1,1,1,0,2)$ & $(0,3 / 2) \oplus(0,5 / 2)$ \\
\hline$(0,0,0,0,0,1,1,1,1,2)$ & $0,1 / 2) \oplus 2(0,3 / 2) \oplus(0,5 / 2)$ & $(0,0,0,0,0,1,2,0,0,2)$ & $(0,1 / 2) \oplus(0,3 / 2) \oplus(0,5 / 2)$ \\
\hline$(0,0,0,0,0,1,2,0,1,2)$ & $(0,1 / 2) \oplus 2(0,3 / 2) \oplus(0,5 / 2)$ & $(0,0,0,0,0,1,2,1,0,2)$ & $(0,1 / 2) \oplus 2(0,3 / 2) \oplus(0,5 / 2)$ \\
\hline$(0,0,0,0,0,1,3,0,0,2)$ & $\begin{array}{l}(0,1 / 2) \oplus 2(0,3 / 2) \oplus 2(0,5 / 2) \oplus \\
(0,7 / 2)\end{array}$ & $(0,0,0,0,0,2,0,0,0,2)$ & $(0,5 / 2) \oplus(0,7 / 2) \oplus(1 / 2,4)$ \\
\hline$(0,0,0,0,0,2,0,0,1,2)$ & $\begin{array}{l}(0,3 / 2) \oplus 3(0,5 / 2) \oplus 2(0,7 / 2) \oplus \\
(1 / 2,3) \oplus(1 / 2,4)\end{array}$ & $(0,0$, & $\begin{array}{l}(0,1 / 2) \oplus 3(0,3 / 2) \oplus 4(0,5 / 2) \oplus \\
2(0,7 / 2) \oplus(1 / 2,2) \oplus(1 / 2,3) \oplus \\
(1 / 2,4)\end{array}$ \\
\hline$(0,0,0,0,0,2,1,0,0,2)$ & $\begin{array}{l}(0,3 / 2) \oplus 3(0,5 / 2) \oplus 2(0,7 / 2) \oplus \\
(1 / 2,3) \oplus(1 / 2,4)\end{array}$ & $(0,0,0,0,0,2,1$ & $\begin{array}{l}(0,1 / 2) \oplus 5(0,3 / 2) \oplus 7(0,5 / 2) \oplus \\
3(0,7 / 2) \oplus(1 / 2,2) \oplus 2(1 / 2,3) \oplus \\
(1 / 2,4)\end{array}$ \\
\hline$(0,0$ & $\begin{array}{l}(0,3 / 2) \oplus 3(0,5 / 2) \oplus 2(0,7 / 2) \oplus \\
(1 / 2,3) \oplus(1 / 2,4)\end{array}$ & $(0$, & $\begin{array}{l}(0,1 / 2) \oplus 3(0,3 / 2) \oplus 4(0,5 / 2) \oplus \\
2(0,7 / 2) \oplus(1 / 2,2) \oplus(1 / 2,3) \oplus \\
(1 / 2,4)\end{array}$ \\
\hline$(0,0,0,0,0,3,0,0,0,2)$ & $\begin{array}{l}(0,5 / 2) \oplus(0,7 / 2) \oplus 2(0,9 / 2) \oplus \\
(1 / 2,4) \oplus(1 / 2,5) \oplus(1,11 / 2)\end{array}$ & $(0,0,0,0,0,3,0,0,1,2)$ & $\begin{array}{l}(0,3 / 2) \oplus 3(0,5 / 2) \oplus 5(0,7 / 2) \oplus \\
3(0,9 / 2) \oplus(1 / 2,3) \oplus 3(1 / 2,4) \oplus \\
2(1 / 2,5) \oplus(1,9 / 2) \oplus(1,11 / 2)\end{array}$ \\
\hline$(0,0,0,0,0,3,1,0,0,2)$ & $\begin{array}{l}(0,3 / 2) \oplus 3(0,5 / 2) \oplus 5(0,7 / 2) \oplus \\
3(0,9 / 2) \oplus(1 / 2,3) \oplus 3(1 / 2,4) \oplus \\
2(1 / 2,5) \oplus(1,9 / 2) \oplus(1,11 / 2)\end{array}$ & $(0,0,0,0,0,4,0,0,0,2)$ & $\begin{array}{l}(0,5 / 2) \oplus(0,7 / 2) \oplus 2(0,9 / 2) \oplus \\
2(0,11 / 2) \oplus(1 / 2,4) \oplus \\
(1 / 2,5) \oplus 2(1 / 2,6) \oplus(1,11 / 2) \oplus \\
(1,13 / 2) \oplus(3 / 2,7)\end{array}$ \\
\hline$(0,0,0,0,1,1,0,0$ & $, 3 / 2) \oplus(0,5 / 2)$ & $(0,0,0,0,1,1,0,0,1,2)$ & $(0,1 / 2) \oplus 2(0,3 / 2) \oplus(0,5 / 2)$ \\
\hline$(0,0,0,0,1,1,0,0,2,2)$ & $(0,1 / 2) \oplus 2(0,3 / 2) \oplus(0,5 / 2)$ & $(0,0,0,0,1,1,1,0,0,2)$ & $(0,1 / 2) \oplus 2(0,3 / 2) \oplus(0,5 / 2)$ \\
\hline$(0,0,0,0,1,1,1,0,1,2)$ & $(0,1 / 2) \oplus 3(0,3 / 2) \oplus(0,5 / 2)$ & $(0,0,0,0,1,1,1,1,0,2)$ & $(0,1 / 2) \oplus 2(0,3 / 2) \oplus(0,5 / 2)$ \\
\hline$(0,0,0,0,1,1,2,0,0,2)$ & $2(0,1 / 2) \oplus 2(0,3 / 2) \oplus(0,5 / 2)$ & $(0,0,0,0,1,2,0,0,0,2)$ & $\begin{array}{l}(0,3 / 2) \oplus 3(0,5 / 2) \oplus 2(0,7 / 2) \oplus \\
(1 / 2,3) \oplus(1 / 2,4)\end{array}$ \\
\hline$, 1,2,0,0,1,2)$ & $\begin{array}{l}(0,1 / 2) \oplus 5(0,3 / 2) \oplus 7(0,5 / 2) \oplus \\
3(0,7 / 2) \oplus(1 / 2,2) \oplus 2(1 / 2,3) \oplus \\
(1 / 2,4)\end{array}$ & $, 0,1,2,1,0,0,2)$ & $\begin{array}{l}(0,1 / 2) \oplus 5(0,3 / 2) \oplus 7(0,5 / 2) \oplus \\
3(0,7 / 2) \oplus(1 / 2,2) \oplus 2(1 / 2,3) \oplus \\
(1 / 2,4)\end{array}$ \\
\hline$(0,0,0,0,1,3,0,0,0,2)$ & $\begin{array}{l}(0,3 / 2) \oplus 3(0,5 / 2) \oplus 5(0,7 / 2) \oplus \\
3(0,9 / 2) \oplus(1 / 2,3) \oplus 3(1 / 2,4) \oplus \\
2(1 / 2,5) \oplus(1,9 / 2) \oplus(1,11 / 2)\end{array}$ & $(0,0,0,0,2,1,0,0,0,2)$ & $(0,1 / 2) \oplus(0,3 / 2) \oplus(0,5 / 2)$ \\
\hline$(0,0,0,0,2,1,0,0,1,2)$ & $(0,1 / 2) \oplus 2(0,3 / 2) \oplus(0,5 / 2)$ & $(0,0,0,0,2,1,1,0,0,2)$ & $2(0,1 / 2) \oplus$ \\
\hline$(0,0,0,0,2,2,0,0,0,2)$ & $\begin{array}{l}(0,1 / 2) \oplus 3(0,3 / 2) \oplus 4(0,5 / 2) \oplus \\
2(0,7 / 2) \oplus(1 / 2,2) \oplus(1 / 2,3) \oplus \\
(1 / 2,4)\end{array}$ & $(0,0,0,0,3,0,0,0,0,2)$ & $(0,5 / 2)$ \\
\hline $0,3,1,0,0,0,2)$ & $\begin{array}{l}(0,1 / 2) \oplus 2(0,3 / 2) \oplus 2(0,5 / 2) \oplus \\
(0,7 / 2)\end{array}$ & $(0,0,0,0,4,0,0,0,0,2)$ & $(0,5 / 2) \oplus(0,7 / 2) \oplus(1 / 2,4)$ \\
\hline$(0,0,0,1,1,1,0,0,0,2)$ & $(0,3 / 2) \oplus(0,5 / 2)$ & $(0,0,0,1,1,1,0,0,1,2)$ & $(0,1 / 2) \oplus 2(0,3 / 2) \oplus(0,5 / 2)$ \\
\hline$(0,0,0,1,1,1,1,0,0,2)$ & $(0,1 / 2) \oplus 2(0,3 / 2) \oplus(0,5 / 2)$ & $(0,0,0,1,1,2,0,0,0,2)$ & $\begin{array}{l}(0,3 / 2) \oplus 3(0,5 / 2) \oplus 2(0,7 / 2) \oplus \\
(1 / 2,3) \oplus(1 / 2,4)\end{array}$ \\
\hline$(0,0,0,1,2,1,0,0,0,2)$ & $(0,1 / 2) \oplus 2(0,3 / 2) \oplus(0,5 / 2)$ & $(0,0,0,1,3,0,0,0,0,2)$ & $(0,3 / 2) \oplus(0,5 / 2)$ \\
\hline$(0,0,1,1,1,1,0,0,0,2)$ & $(0,3 / 2) \oplus(0,5 / 2)$ & $(0,0,0,0,0,1,0,0,0,3)$ & $(0,7 / 2)$ \\
\hline$(0,0,0,0,0,1,0,0,1,3)$ & $(0,5 / 2) \oplus(0,7 / 2)$ & $(0,0,0,0,0,1,0,0,2,3)$ & $(0,3 / 2) \oplus(0,5 / 2) \oplus(0,7 / 2)$ \\
\hline
\end{tabular}




\begin{tabular}{|c|l|l|l|}
\hline$\beta$ & \multicolumn{1}{|c|}{$\oplus N_{j_{l}, j_{r}}^{\beta}\left(j_{l}, j_{r}\right)$} & \multicolumn{1}{|c|}{$\beta$} & \multicolumn{1}{c|}{$\oplus N_{j_{l}, j_{r}}^{\beta}\left(j_{l}, j_{r}\right)$} \\
\hline$(0,0,0,0,0,1,1,0,0,3)$ & $(0,5 / 2) \oplus(0,7 / 2)$ & $(0,0,0,0,0,1,1,0,1,3)$ & $(0,3 / 2) \oplus 2(0,5 / 2) \oplus(0,7 / 2)$ \\
\hline$(0,0,0,0,0,1,1,1,0,3)$ & $(0,5 / 2) \oplus(0,7 / 2)$ & $(0,0,0,0,0,1,2,0,0,3)$ & $(0,3 / 2) \oplus(0,5 / 2) \oplus(0,7 / 2)$ \\
\hline$(0,0,0,0,0,2,0,0,0,3)$ & $(0,5 / 2) \oplus(0,7 / 2) \oplus 2(0,9 / 2) \oplus$ & $(0,0,0,0,0,2,0,0,1,3)$ & $(0,3 / 2) \oplus 3(0,5 / 2) \oplus 5(0,7 / 2) \oplus$ \\
& $(1 / 2,4) \oplus(1 / 2,5) \oplus(1,11 / 2)$ & & $3(0,9 / 2) \oplus(1 / 2,3) \oplus 3(1 / 2,4) \oplus$ \\
& & & $2(1 / 2,5) \oplus(1,9 / 2) \oplus(1,11 / 2)$ \\
\hline$(0,0,0,0,0,2,1,0,0,3)$ & $(0,3 / 2) \oplus 3(0,5 / 2) \oplus 5(0,7 / 2) \oplus$ & $(0,0,0,0,0,3,0,0,0,3)$ & $(0,3 / 2) \oplus(0,5 / 2) \oplus 3(0,7 / 2) \oplus$ \\
& $3(0,9 / 2) \oplus(1 / 2,3) \oplus 3(1 / 2,4) \oplus$ & & $3(0,9 / 2) \oplus 4(0,11 / 2) \oplus$ \\
& $2(1 / 2,5) \oplus(1,9 / 2) \oplus(1,11 / 2)$ & & $3(1 / 2,3) \oplus 2(1 / 2,4) \oplus 3(1 / 2,5) \oplus$ \\
& & & $2(1,11 / 2) \oplus 3(1,13 / 2) \oplus$ \\
& & & $(3 / 2,6) \oplus(3 / 2,7) \oplus(2,15 / 2)$ \\
\hline$(0,0,0,0,1,1,0,0,0,3)$ & $(0,5 / 2) \oplus(0,7 / 2)$ & $(0,0,0,0,1,1,0,0,1,3)$ & $(0,3 / 2) \oplus 2(0,5 / 2) \oplus(0,7 / 2)$ \\
\hline$(0,0,0,0,1,1,1,0,0,3)$ & $(0,3 / 2) \oplus 2(0,5 / 2) \oplus(0,7 / 2)$ & $(0,0,0,0,1,2,0,0,0,3)$ & $(0,3 / 2) \oplus 3(0,5 / 2) \oplus 5(0,7 / 2) \oplus$ \\
& & & $3(0,9 / 2) \oplus(1 / 2,3) \oplus 3(1 / 2,4) \oplus$ \\
& & & $2(1 / 2,5) \oplus(1,9 / 2) \oplus(1,11 / 2)$ \\
\hline$(0,0,0,0,2,1,0,0,0,3)$ & $(0,3 / 2) \oplus(0,5 / 2) \oplus(0,7 / 2)$ & $(0,0,0,1,1,1,0,0,0,3)$ & $(0,5 / 2) \oplus(0,7 / 2)$ \\
\hline$(0,0,0,0,0,1,0,0,0,4)$ & $(0,9 / 2)$ & $(0,0,0,0,0,1,0,0,1,4)$ & $(0,7 / 2) \oplus(0,9 / 2)$ \\
\hline$(0,0,0,0,0,1,1,0,0,4)$ & $(0,7 / 2) \oplus(0,9 / 2)$ & $(0,0,0,0,0,2,0,0,0,4)$ & $(0,5 / 2) \oplus(0,7 / 2) \oplus 2(0,9 / 2) \oplus$ \\
& & & $2(0,11 / 2) \oplus(1 / 2,4) \oplus$ \\
& & & $(1 / 2,5) \oplus 2(1 / 2,6) \oplus(1,11 / 2) \oplus$ \\
\hline$(0,0,0,0,1,1,0,0,0,4)$ & $(0,7 / 2) \oplus(0,9 / 2)$ & $(1,13 / 2) \oplus(3 / 2,7)$ \\
\hline
\end{tabular}

Table 27. Refined BPS invariants of $6 \mathrm{~d} E_{8}$ minimal SCFT. 


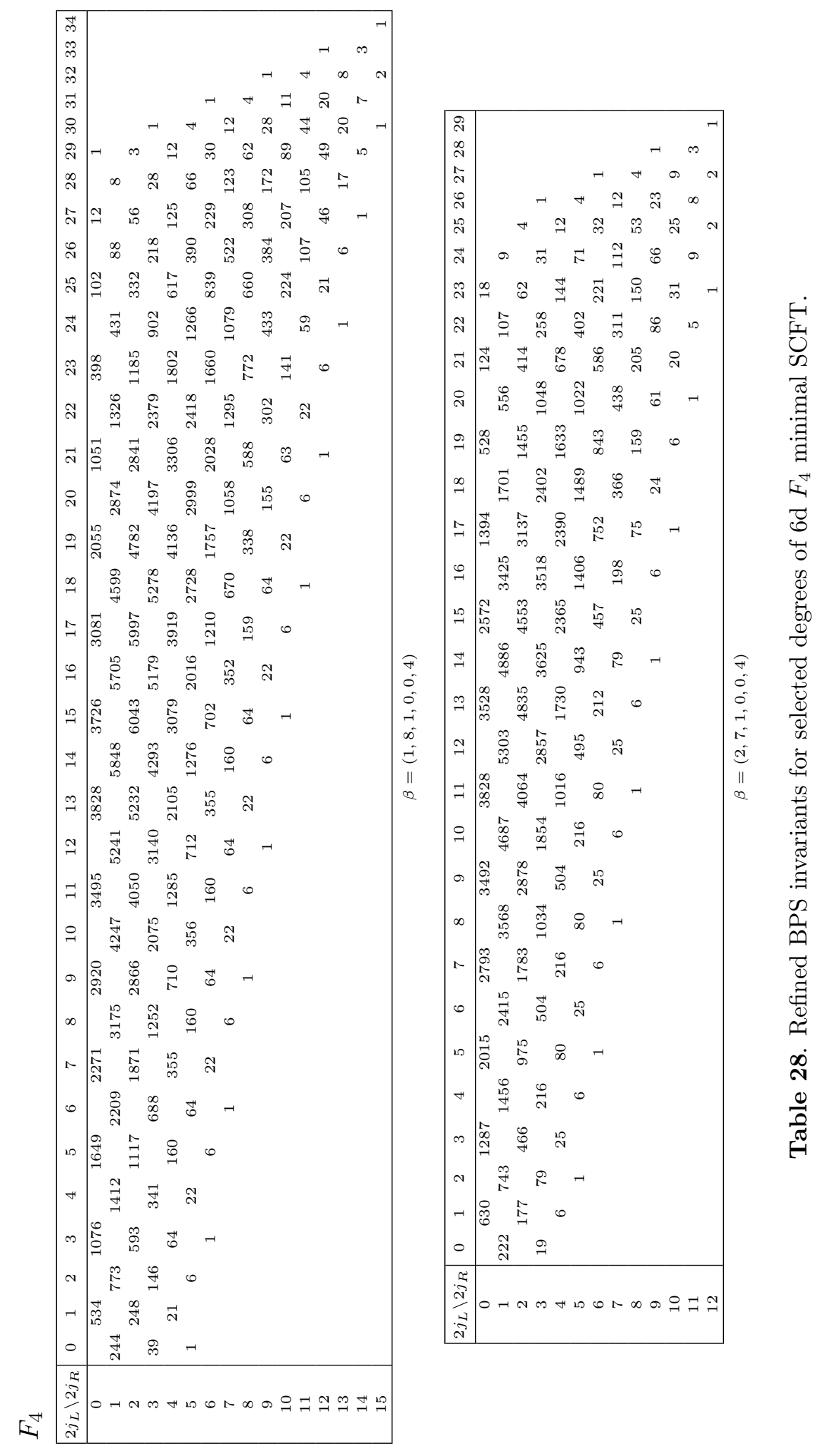



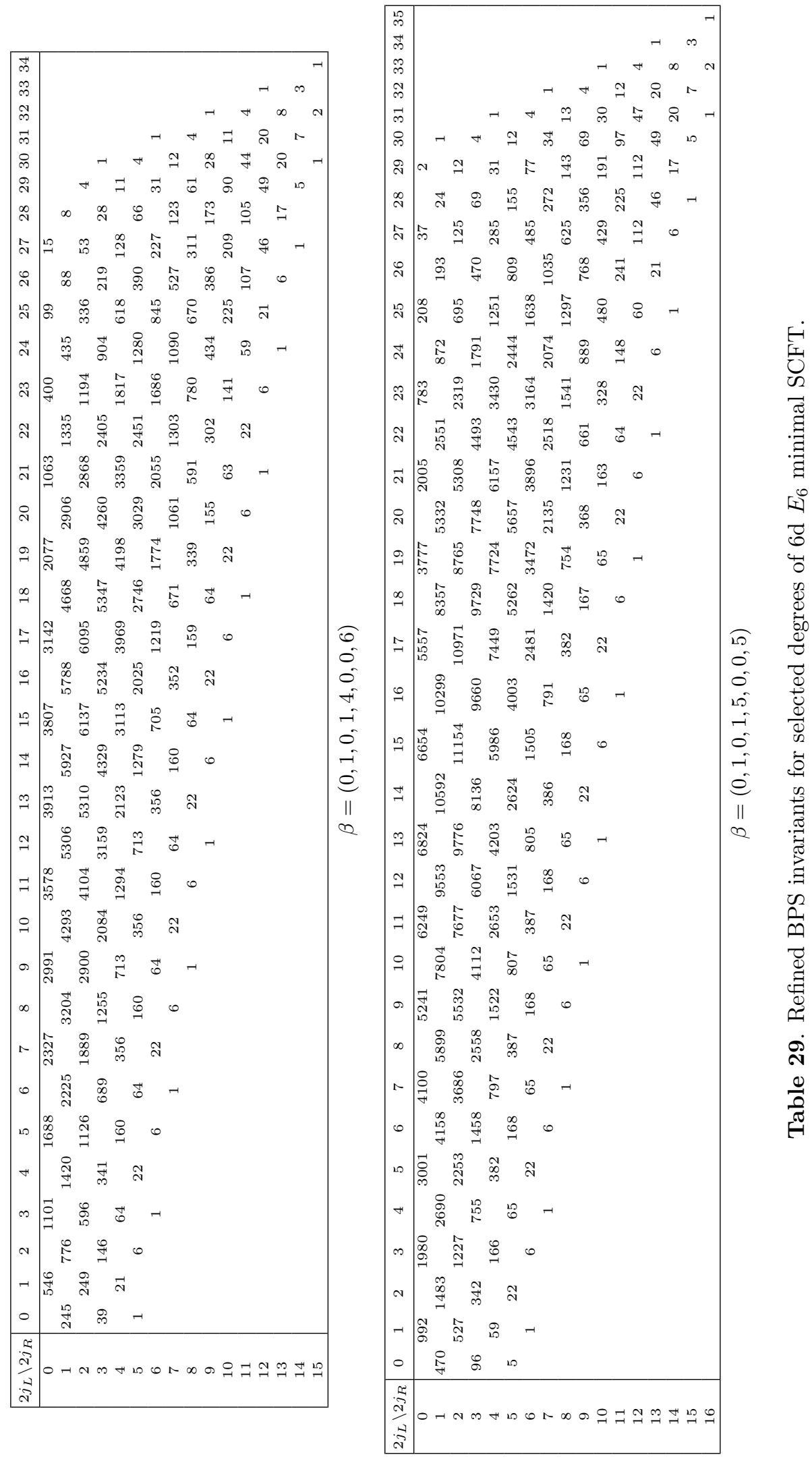

성

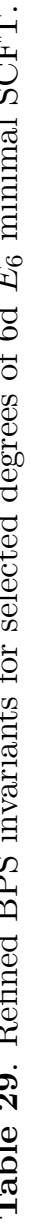




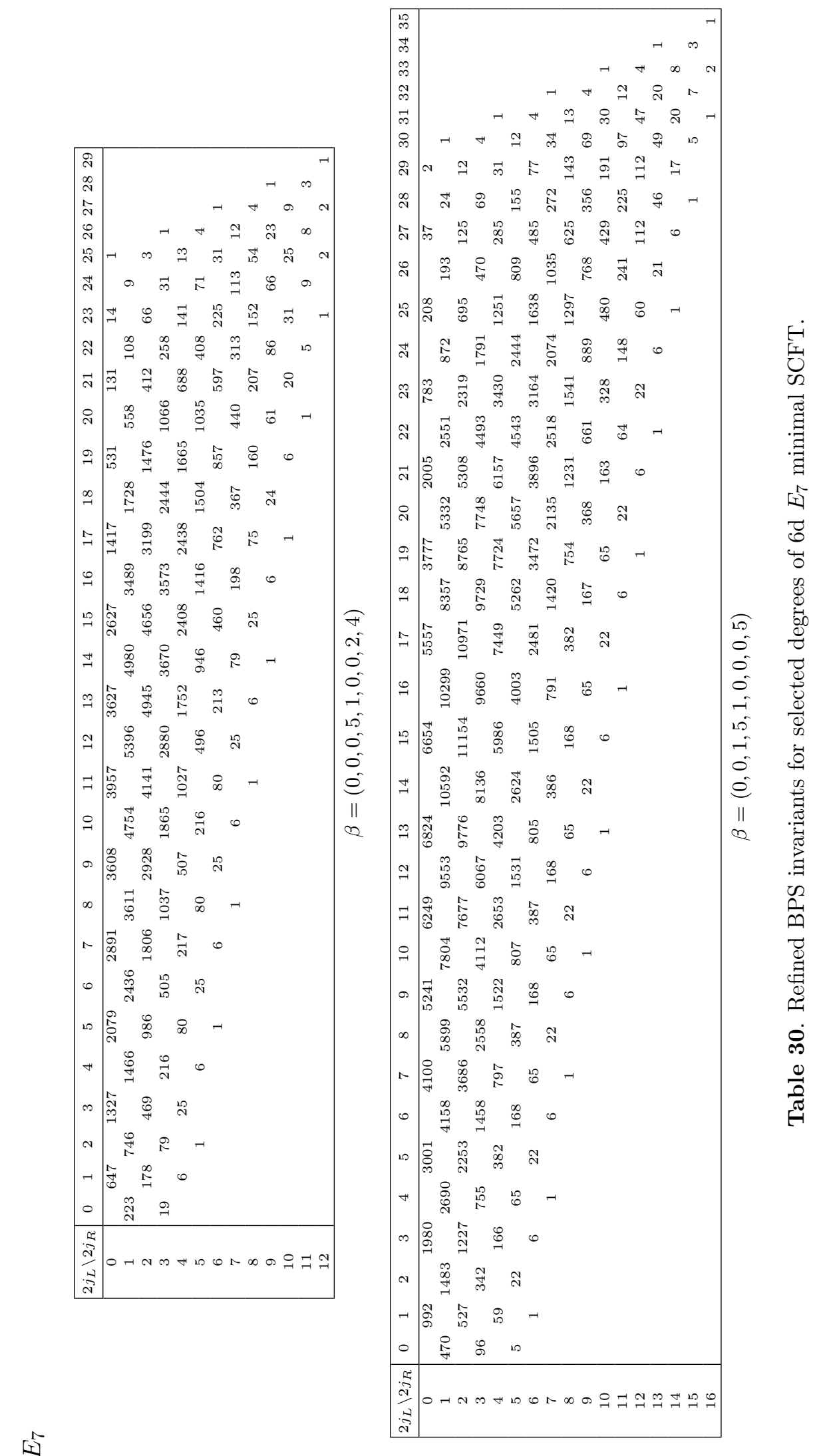


$E_{8}$

\begin{tabular}{|c|c|c|c|c|c|c|c|c|c|c|c|c|c|c|c|c|c|c|c|c|}
\hline $2 j_{L} \backslash 2 j_{R}$ & 0 & 1 & 2 & 3 & 4 & 5 & 6 & 7 & 8 & 9 & 10 & 11 & 12 & 13 & 14 & 15 & 16 & 17 & 18 & 19 \\
\hline 0 & & 3 & & 7 & & 13 & & 21 & & 27 & & 28 & & 17 & & 4 & & 1 & & \\
\hline 1 & & & 1 & & 4 & & 10 & & 19 & & 28 & & 31 & & 19 & & 4 & & & \\
\hline 2 & & & & & & 1 & & 4 & & 11 & & 20 & & 25 & & 15 & & 2 & & \\
\hline 3 & & & & & & & & & 1 & & 4 & & 10 & & 15 & & 9 & & 1 & \\
\hline 4 & & & & & & & & & & & & 1 & & 4 & & 8 & & 5 & & \\
\hline 5 & & & & & & & & & & & & & & & 1 & & 3 & & 2 & \\
\hline 6 & & & & & & & & & & & & & & & & & & 1 & & 1 \\
\hline
\end{tabular}

$\beta=(1,1,1,1,1,3,0,0,0,4)$

\begin{tabular}{|c|cccccccccccccccc|}
\hline $2 j_{L} \backslash 2 j_{R}$ & 0 & 1 & 2 & 3 & 4 & 5 & 6 & 7 & 8 & 9 & 10 & 11 & 12 & 13 & 14 & 15 \\
\hline 0 & & 17 & & 44 & & 79 & & 91 & & 63 & & 23 & & 2 & & \\
1 & 1 & & 6 & & 22 & & 51 & & 68 & & 50 & & 17 & & 1 & \\
2 & & & & 1 & & 6 & & 20 & & 31 & & 25 & & 9 & & \\
3 & & & & & & & 1 & & 5 & & 9 & & 8 & & 3 & \\
4 & & & & & & & & & & 1 & & 2 & & 2 & & 1 \\
\hline
\end{tabular}

$\beta=(0,1,1,1,1,3,0,0,2,3)$

Table 31. Refined BPS invariants for selected degrees of $6 \mathrm{~d} E_{8}$ minimal SCFT.

Open Access. This article is distributed under the terms of the Creative Commons Attribution License (CC-BY 4.0), which permits any use, distribution and reproduction in any medium, provided the original author(s) and source are credited.

\section{References}

[1] W. Nahm, Supersymmetries and their Representations, Nucl. Phys. B 135 (1978) 149 [INSPIRE].

[2] O.J. Ganor and A. Hanany, Small E $E_{8}$ instantons and tensionless noncritical strings, Nucl. Phys. B 474 (1996) 122 [hep-th/9602120] [INSPIRE].

[3] A. Klemm, P. Mayr and C. Vafa, BPS states of exceptional noncritical strings, Nucl. Phys. Proc. Suppl. 58 (1997) 177 [hep-th/9607139] [INSPIRE].

[4] D.R. Morrison and C. Vafa, Compactifications of F-theory on Calabi-Yau threefolds. 2., Nucl. Phys. B 476 (1996) 437 [hep-th/9603161] [INSPIRE].

[5] J.J. Heckman, D.R. Morrison and C. Vafa, On the Classification of 6D SCFTs and Generalized ADE Orbifolds, JHEP 05 (2014) 028 [Erratum ibid. 06 (2015) 017] [arXiv:1312.5746] [INSPIRE].

[6] J.J. Heckman, D.R. Morrison, T. Rudelius and C. Vafa, Atomic Classification of $6 D$ SCFTs, Fortsch. Phys. 63 (2015) 468 [arXiv: 1502.05405] [INSPIRE]. 
[7] L. Bhardwaj, Classification of $6 d \mathcal{N}=(1,0)$ gauge theories, JHEP 11 (2015) 002 [arXiv: 1502.06594] [INSPIRE].

[8] B. Haghighat, A. Klemm, G. Lockhart and C. Vafa, Strings of Minimal 6d SCFTs, Fortsch. Phys. 63 (2015) 294 [arXiv:1412.3152] [INSPIRE].

[9] R. Pandharipande and R.P. Thomas, Curve counting via stable pairs in the derived category, Invent. Math. 178 (2009) 407.

[10] R. Pandharipande and R.P. Thomas, Stable pairs and BPS invariants, J. Am. Math. Soc. 23 (2010) 267.

[11] J. Choi, S. Katz and A. Klemm, The refined BPS index from stable pair invariants, Commun. Math. Phys. 328 (2014) 903 [arXiv:1210.4403] [InSPIRE].

[12] N. Nekrasov and A. Okounkov, Membranes and sheaves, Algebr. Geom. 3 (2016) 320.

[13] A. Iqbal, C. Kozçaz and C. Vafa, The Refined topological vertex, JHEP 10 (2009) 069 [hep-th/0701156] [INSPIRE].

[14] M.-x. Huang and A. Klemm, Direct integration for general $\Omega$ backgrounds, Adv. Theor. Math. Phys. 16 (2012) 805 [arXiv: 1009.1126] [INSPIRE].

[15] M.-x. Huang, K. Sun and X. Wang, Blowup Equations for Refined Topological Strings, JHEP 10 (2018) 196 [arXiv:1711.09884] [INSPIRE].

[16] H. Nakajima and K. Yoshioka, Instanton counting on blowup. II. K-theoretic partition function, math/0505553.

[17] L. Gottsche, H. Nakajima and K. Yoshioka, K-theoretic Donaldson invariants via instanton counting, Pure Appl. Math. Quart. 5 (2009) 1029 [math/0611945] [INSPIRE].

[18] H. Nakajima and K. Yoshioka, Perverse coherent sheaves on blowup, III: Blow-up formula from wall-crossing, Kyoto J. Math. 51 (2011) 263.

[19] B. Haghighat, A. Iqbal, C. Kozçaz, G. Lockhart and C. Vafa, M-Strings, Commun. Math. Phys. 334 (2015) 779 [arXiv: 1305.6322] [InSPIRE].

[20] A. Gadde and S. Gukov, 2d Index and Surface operators, JHEP 03 (2014) 080 [arXiv: 1305.0266] [INSPIRE].

[21] F. Benini, R. Eager, K. Hori and Y. Tachikawa, Elliptic Genera of $2 d \mathcal{N}=2$ Gauge Theories, Commun. Math. Phys. 333 (2015) 1241 [arXiv:1308.4896] [InSPIRE].

[22] M.-x. Huang, S. Katz and A. Klemm, Topological String on elliptic CY 3-folds and the ring of Jacobi forms, JHEP 10 (2015) 125 [arXiv: 1501.04891] [INSPIRE].

[23] J. Gu, M.-x. Huang, A.-K. Kashani-Poor and A. Klemm, Refined BPS invariants of $6 d$ SCFTs from anomalies and modularity, JHEP 05 (2017) 130 [arXiv:1701.00764] [INSPIRE].

[24] M. Del Zotto, J. Gu, M.-X. Huang, A.-K. Kashani-Poor, A. Klemm and G. Lockhart, Topological Strings on Singular Elliptic Calabi-Yau 3-folds and Minimal 6d SCFTs, JHEP 03 (2018) 156 [arXiv:1712.07017] [INSPIRE].

[25] J. Gu, B. Haghighat, K. Sun and X. Wang, Blowup Equations for 6d SCFTs. I, JHEP 03 (2019) 002 [arXiv:1811.02577] [INSPIRE].

[26] S.H. Katz, A. Klemm and C. Vafa, Geometric engineering of quantum field theories, Nucl. Phys. B 497 (1997) 173 [hep-th/9609239] [INSPIRE]. 
[27] N.A. Nekrasov, Seiberg-Witten prepotential from instanton counting, Adv. Theor. Math. Phys. 7 (2003) 831 [hep-th/0206161] [INSPIRE].

[28] H. Nakajima and K. Yoshioka, Instanton counting on blowup. 1., Invent. Math. 162 (2005) 313 [math/0306198].

[29] A. Grassi and J. Gu, BPS relations from spectral problems and blowup equations, Lett. Math. Phys. 109 (2019) 1271 [arXiv:1609.05914] [INSPIRE].

[30] C.A. Keller and J. Song, Counting Exceptional Instantons, JHEP 07 (2012) 085 [arXiv: 1205.4722] [INSPIRE].

[31] http://www.th.physik.uni-bonn.de/klemm/data.php.

[32] J. Kim, S. Kim, K. Lee, J. Park and C. Vafa, Elliptic Genus of E-strings, JHEP 09 (2017) 098 [arXiv: 1411.2324] [INSPIRE].

[33] J. Kim, S. Kim and K. Lee, Higgsing towards E-strings, arXiv:1510.03128 [INSPIRE].

[34] H.-C. Kim, S. Kim and J. Park, 6d strings from new chiral gauge theories, arXiv: 1608.03919 [INSPIRE].

[35] M.-X. Huang, A. Klemm and M. Poretschkin, Refined stable pair invariants for E-, $M$ - and [p,q]-strings, JHEP 11 (2013) 112 [arXiv:1308.0619] [INSPIRE].

[36] Z. Duan, J. Gu and A.-K. Kashani-Poor, Computing the elliptic genus of higher rank E-strings from genus $0 G W$ invariants, JHEP 03 (2019) 078 [arXiv:1810.01280] [INSPIRE].

[37] M. Del Zotto and G. Lockhart, On Exceptional Instanton Strings, JHEP 09 (2017) 081 [arXiv: 1609.00310] [INSPIRE].

[38] M. Del Zotto and G. Lockhart, Universal Features of BPS Strings in Six-dimensional SCFTs, JHEP 08 (2018) 173 [arXiv: 1804.09694] [INSPIRE].

[39] S.-S. Kim, M. Taki and F. Yagi, Tao Probing the End of the World, PTEP 2015 (2015) 083B02 [arXiv: 1504.03672] [INSPIRE].

[40] H. Hayashi and K. Ohmori, 5d/6d DE instantons from trivalent gluing of web diagrams, JHEP 06 (2017) 078 [arXiv: 1702.07263] [INSPIRE].

[41] S. Benvenuti, A. Hanany and N. Mekareeya, The Hilbert Series of the One Instanton Moduli Space, JHEP 06 (2010) 100 [arXiv: 1005.3026] [INSPIRE].

[42] A. Hanany, N. Mekareeya and S.S. Razamat, Hilbert Series for Moduli Spaces of Two Instantons, JHEP 01 (2013) 070 [arXiv: 1205.4741] [INSPIRE].

[43] S. Cremonesi, G. Ferlito, A. Hanany and N. Mekareeya, Coulomb Branch and The Moduli Space of Instantons, JHEP 12 (2014) 103 [arXiv:1408.6835] [INSPIRE].

[44] A. Gadde, L. Rastelli, S.S. Razamat and W. Yan, Gauge Theories and Macdonald Polynomials, Commun. Math. Phys. 319 (2013) 147 [arXiv:1110.3740] [INSPIRE].

[45] D. Gaiotto and S.S. Razamat, Exceptional Indices, JHEP 05 (2012) 145 [arXiv:1203.5517] [INSPIRE].

[46] P. Putrov, J. Song and W. Yan, (0,4) dualities, JHEP 03 (2016) 185 [arXiv:1505.07110] [INSPIRE].

[47] A. Gadde, S.S. Razamat and B. Willett, "Lagrangian" for a Non-Lagrangian Field Theory with $\mathcal{N}=2$ Supersymmetry, Phys. Rev. Lett. 115 (2015) 171604 [arXiv:1505.05834] [INSPIRE]. 
[48] P. Agarwal, K. Maruyoshi and J. Song, A "Lagrangian" for the $E_{7}$ superconformal theory, JHEP 05 (2018) 193 [arXiv: 1802.05268] [INSPIRE].

[49] B. Haghighat, G. Lockhart and C. Vafa, Fusing E-strings to heterotic strings: $E+E \rightarrow H$, Phys. Rev. D 90 (2014) 126012 [arXiv:1406.0850] [INSPIRE].

[50] W. Cai, M.-x. Huang and K. Sun, On the Elliptic Genus of Three E-strings and Heterotic Strings, JHEP 01 (2015) 079 [arXiv:1411.2801] [INSPIRE].

[51] J. Gu, B. Haghighat, A. Klemm, K. Sun and X. Wang, Elliptic Blowup Equations for 6d SCFTs. IV: Matters, to appear.

[52] J. Gu, B. Haghighat, A. Klemm, K. Sun and X. Wang, Elliptic Blowup Equations for 6d SCFTs. III: E-strings, M-strings and Chains, to appear.

[53] M. Del Zotto, J.J. Heckman and D.R. Morrison, 6D SCFTs and Phases of $5 D$ Theories, JHEP 09 (2017) 147 [arXiv: 1703.02981] [InSPIRE].

[54] M.-x. Huang, A.-K. Kashani-Poor and A. Klemm, The $\Omega$ deformed B-model for rigid $\mathcal{N}=2$ theories, Annales Henri Poincaré 14 (2013) 425 [arXiv:1109.5728] [INSPIRE].

[55] M. Esole, P. Jefferson and M.J. Kang, The Geometry of F -Models, arXiv:1704.08251 [INSPIRE].

[56] S. Hosono, A. Klemm and S. Theisen, Lectures on mirror symmetry, Lect. Notes Phys. 436 (1994) 235 [hep-th/9403096] [INSPIRE].

[57] S. Hosono, A. Klemm, S. Theisen and S.-T. Yau, Mirror symmetry, mirror map and applications to Calabi-Yau hypersurfaces, Commun. Math. Phys. 167 (1995) 301 [hep-th/9308122] [INSPIRE].

[58] T. Schimannek, Modularity from Monodromy, JHEP 05 (2019) 024 [arXiv:1902.08215] [INSPIRE].

[59] C.A. Keller, N. Mekareeya, J. Song and Y. Tachikawa, The ABCDEFG of Instantons and $W$-algebras, JHEP 03 (2012) 045 [arXiv:1111.5624] [INSPIRE].

[60] A. Hanany and R. Kalveks, Highest Weight Generating Functions for Hilbert Series, JHEP 10 (2014) 152 [arXiv:1408.4690] [INSPIRE].

[61] P. Di Francesco, P. Mathieu and D. Senechal, Conformal Field Theory, Graduate Texts in Contemporary Physics. Springer-Verlag, New York (1997) [DOI:10.1007/978-1-4612-2256-9].

[62] P.C. Argyres, M.R. Plesser, N. Seiberg and E. Witten, New $N=2$ superconformal field theories in four-dimensions, Nucl. Phys. B 461 (1996) 71 [hep-th/9511154] [INSPIRE].

[63] T. Banks, M.R. Douglas and N. Seiberg, Probing F-theory with branes, Phys. Lett. B 387 (1996) 278 [hep-th/9605199] [INSPIRE].

[64] M.R. Douglas, D.A. Lowe and J.H. Schwarz, Probing F-theory with multiple branes, Phys. Lett. B 394 (1997) 297 [hep-th/9612062] [INSPIRE].

[65] J.A. Minahan and D. Nemeschansky, An $N=2$ superconformal fixed point with $E_{6}$ global symmetry, Nucl. Phys. B 482 (1996) 142 [hep-th/9608047] [INSPIRE].

[66] J.A. Minahan and D. Nemeschansky, Superconformal fixed points with E(n) global symmetry, Nucl. Phys. B 489 (1997) 24 [hep-th/9610076] [InSPIRE].

[67] P.C. Argyres and N. Seiberg, S-duality in $N=2$ supersymmetric gauge theories, JHEP 12 (2007) 088 [arXiv:0711.0054] [INSPIRE]. 
[68] A. Kapustin, Holomorphic reduction of $N=2$ gauge theories, Wilson-'t Hooft operators and S-duality, hep-th/0612119 [INSPIRE].

[69] V.P. Spiridonov and S.O. Warnaar, Inversions of integral operators and elliptic beta integrals on root systems, Adv. Math. 207 (2006) 91.

[70] F. Benini, S. Benvenuti and Y. Tachikawa, Webs of five-branes and $N=2$ superconformal field theories, JHEP 09 (2009) 052 [arXiv:0906.0359] [INSPIRE].

[71] D. Xie, General Argyres-Douglas Theory, JHEP 01 (2013) 100 [arXiv:1204.2270] [InSPIRE].

[72] G. Bonelli, K. Maruyoshi and A. Tanzini, Wild Quiver Gauge Theories, JHEP 02 (2012) 031 [arXiv:1112.1691] [INSPIRE].

[73] C. Beem, M. Lemos, P. Liendo, W. Peelaers, L. Rastelli and B.C. van Rees, Infinite Chiral Symmetry in Four Dimensions, Commun. Math. Phys. 336 (2015) 1359 [arXiv:1312.5344] [INSPIRE].

[74] C. Beem, W. Peelaers, L. Rastelli and B.C. van Rees, Chiral algebras of class S, JHEP 05 (2015) 020 [arXiv: 1408.6522] [INSPIRE].

[75] Y. Pan and W. Peelaers, Chiral Algebras, Localization and Surface Defects, JHEP 02 (2018) 138 [arXiv: 1710.04306$]$ [INSPIRE].

[76] Y. Pan and W. Peelaers, Schur correlation functions on $S^{3} \times S^{1}$, JHEP 07 (2019) 013 [arXiv: 1903.03623] [INSPIRE].

[77] J. Oh and J. Yagi, Chiral algebras from $\Omega$-deformation, JHEP 08 (2019) 143 [arXiv: 1903.11123] [INSPIRE].

[78] M. Dedushenko and M. Fluder, Chiral Algebra, Localization, Modularity, Surface defects, And All That, arXiv:1904.02704 [INSPIRE].

[79] S. Jeong, SCFT/VOA correspondence via $\Omega$-deformation, JHEP 10 (2019) 171 [arXiv: 1904.00927] [INSPIRE].

[80] R. Eager, G. Lockhart and E. Sharpe, Hidden exceptional symmetry in the pure spinor superstring, arXiv:1902.09504 [INSPIRE].

[81] J. Kinney, J.M. Maldacena, S. Minwalla and S. Raju, An Index for 4 dimensional super conformal theories, Commun. Math. Phys. 275 (2007) 209 [hep-th/0510251] [InSPIRE].

[82] C. Romelsberger, Counting chiral primaries in $N=1, d=4$ superconformal field theories, Nucl. Phys. B 747 (2006) 329 [hep-th/0510060] [INSPIRE].

[83] A. Gadde, L. Rastelli, S.S. Razamat and W. Yan, The 4d Superconformal Index from q-deformed $2 d$ Yang-Mills, Phys. Rev. Lett. 106 (2011) 241602 [arXiv:1104.3850] [INSPIRE].

[84] C. Cordova and S.-H. Shao, Schur Indices, BPS Particles and Argyres-Douglas Theories, JHEP 01 (2016) 040 [arXiv: 1506. 00265] [INSPIRE].

[85] A. Gadde, L. Rastelli, S.S. Razamat and W. Yan, The Superconformal Index of the $E_{6}$ SCFT, JHEP 08 (2010) 107 [arXiv:1003.4244] [INSPIRE].

[86] C. Cordova, D. Gaiotto and S.-H. Shao, Infrared Computations of Defect Schur Indices, JHEP 11 (2016) 106 [arXiv:1606.08429] [INSPIRE].

[87] H.-C. Kim, J. Kim, S. Kim, K.-H. Lee and J. Park, $6 d$ strings and exceptional instantons, arXiv: 1801.03579 [INSPIRE]. 
[88] B. Haghighat, S. Murthy, C. Vafa and S. Vandoren, F-Theory, Spinning Black Holes and Multi-string Branches, JHEP 01 (2016) 009 [arXiv: 1509.00455] [INSPIRE].

[89] H. Hayashi, P. Jefferson, H.-C. Kim, K. Ohmori and C. Vafa, SCFTs, Holography and Topological Strings, arXiv:1905.00116 [INSPIRE].

[90] V. Braun and D.R. Morrison, F-theory on Genus-One Fibrations, JHEP 08 (2014) 132 [arXiv: 1401.7844] [INSPIRE].

[91] D.R. Morrison and W. Taylor, Sections, multisections and U(1) fields in F-theory, arXiv: 1404.1527 [INSPIRE].

[92] J.J. Heckman and T. Rudelius, Top Down Approach to 6D SCFTs, J. Phys. A 52 (2019) 093001 [arXiv: 1805.06467] [INSPIRE].

[93] A. Braverman, M. Finkelberg and H. Nakajima, Instanton moduli spaces and $\mathscr{W}$-algebras, arXiv: 1406.2381 [INSPIRE].

[94] M. Taki, On AGT-W Conjecture and q-Deformed W-Algebra, arXiv:1403.7016 [INSPIRE].

[95] https://www.tfc.tohoku.ac.jp/wp-content/uploads/2018/06/Fukuda.pdf.

[96] A. Klemm, B. Lian, S.S. Roan and S.-T. Yau, Calabi-Yau fourfolds for M-theory and F-theory compactifications, Nucl. Phys. B 518 (1998) 515 [hep-th/9701023] [InSPIRE].

[97] J. Kim, K. Lee and J. Park, On elliptic genera of 6d string theories, JHEP 10 (2018) 100 [arXiv: 1801.01631] [INSPIRE]. 